\title{
The development and evaluation of a smoking cessation referral aid for the primary care setting
}

Citation for published version (APA):

Zijlstra, D. N. (2022). The development and evaluation of a smoking cessation referral aid for the primary care setting. [Doctoral Thesis, Maastricht University]. ProefschriftMaken.

https://doi.org/10.26481/dis.20220210dz

Document status and date:

Published: 01/01/2022

DOI:

$10.26481 /$ dis.20220210dz

Document Version:

Publisher's PDF, also known as Version of record

\section{Please check the document version of this publication:}

- A submitted manuscript is the version of the article upon submission and before peer-review. There can be important differences between the submitted version and the official published version of record.

People interested in the research are advised to contact the author for the final version of the publication, or visit the DOI to the publisher's website.

- The final author version and the galley proof are versions of the publication after peer review.

- The final published version features the final layout of the paper including the volume, issue and page numbers.

Link to publication

\footnotetext{
General rights rights.

- You may freely distribute the URL identifying the publication in the public portal. please follow below link for the End User Agreement:

www.umlib.nl/taverne-license

Take down policy

If you believe that this document breaches copyright please contact us at:

repository@maastrichtuniversity.nl

providing details and we will investigate your claim.
}

Copyright and moral rights for the publications made accessible in the public portal are retained by the authors and/or other copyright owners and it is a condition of accessing publications that users recognise and abide by the legal requirements associated with these

- Users may download and print one copy of any publication from the public portal for the purpose of private study or research.

- You may not further distribute the material or use it for any profit-making activity or commercial gain

If the publication is distributed under the terms of Article $25 \mathrm{fa}$ of the Dutch Copyright Act, indicated by the "Taverne" license above, 


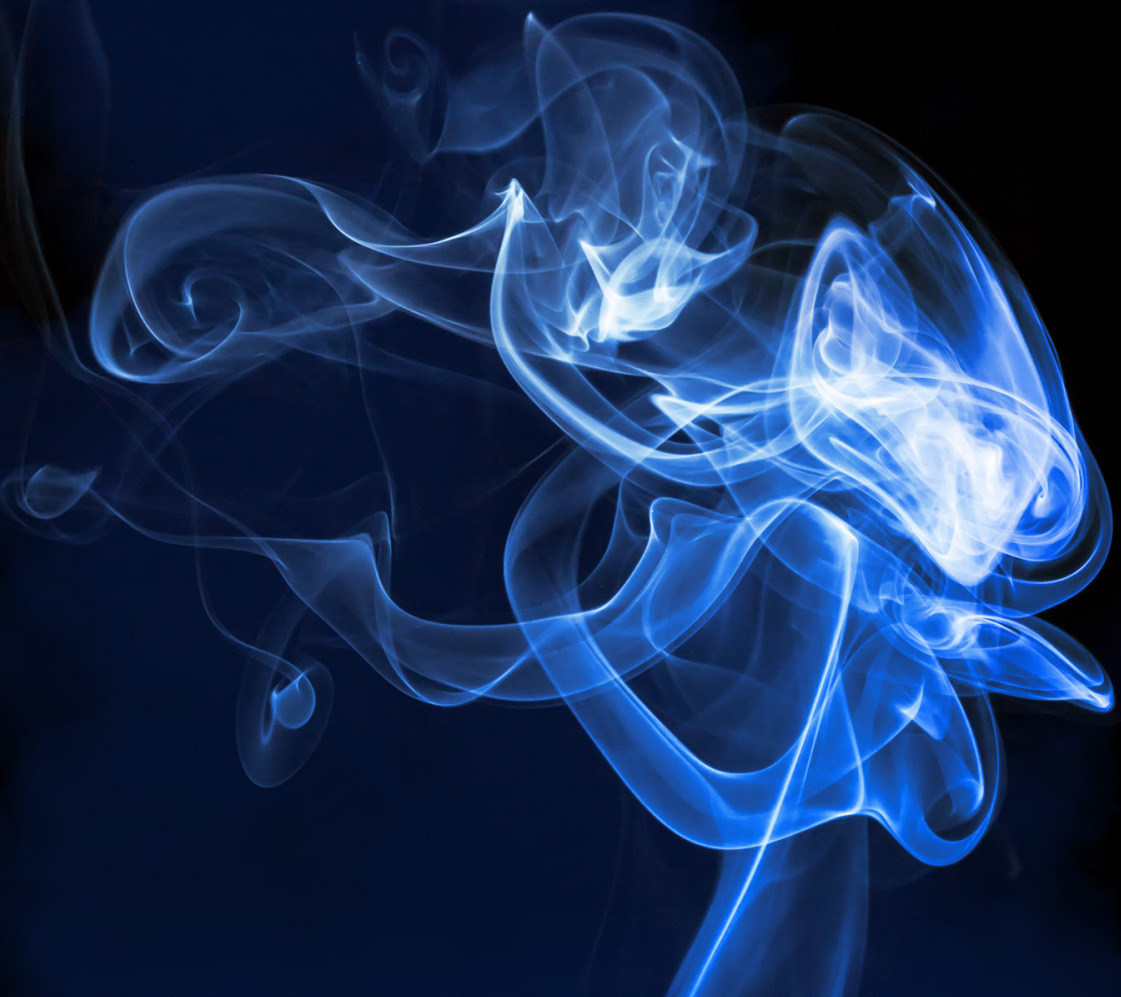

THE DEVELOPMENT AND EVALUATION OF A

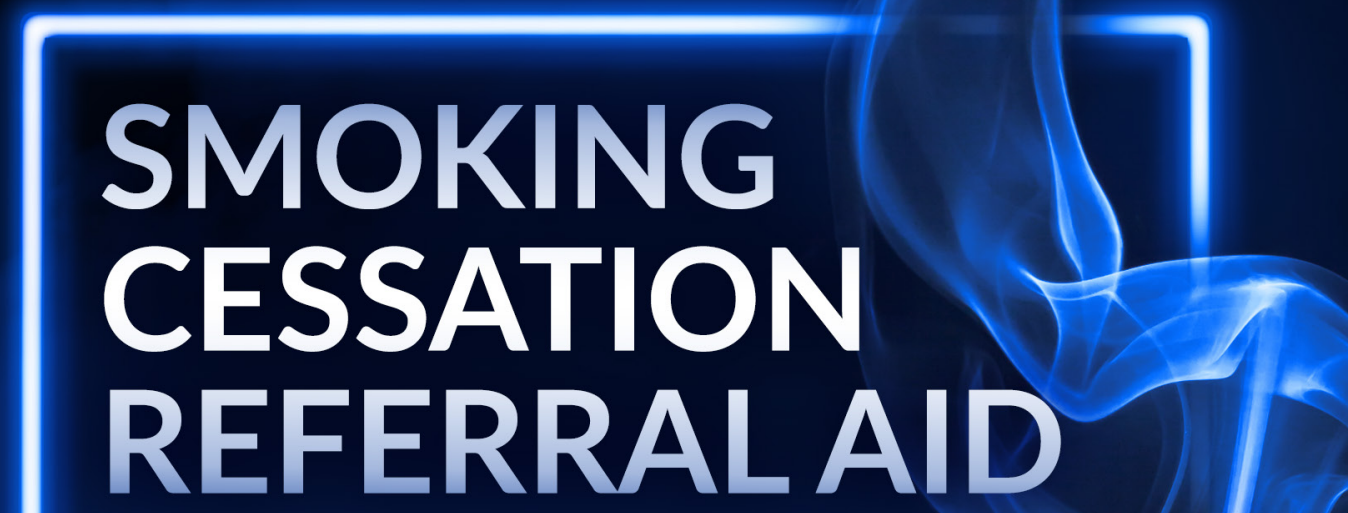

FOR THE PRIMARY CARE SETTING

Daniëlle Nicole Zijlstra 

The development and evaluation of a smoking cessation referral aid for the primary care setting

Daniëlle Nicole Zijlstra 
The research presented in this thesis was conducted at CAPHRI Care and Public Health Research Institute, Department of Health Promotion, of Maastricht University. CAPHRI participates in the Netherlands School of Public Health and Care Research CaRe.

The studies presented in this thesis were conducted with financial support from ZonMW.

ISBN: 978-94-6423-620-0

Design \& lay-out: Wendy Schoneveld ॥ www.wenziD.nl Printed by: ProefschriftMaken II ProefschriftMaken.nl 


\title{
The development and evaluation of a smoking cessation referral aid for the primary care setting
}

\author{
Proefschrift
}

ter verkrijging van de graad van doctor aan de Universiteit Maastricht, op gezag van de Rector Magnificus, Prof. dr. Pamela Habibovic volgens het besluit van het College van Decanen,

in het openbaar te verdedigen

op donderdag 10 februari 2022 om 10.00 uur

door

Daniëlle Nicole Zijlstra 


\section{Promotores}

Prof. Dr. Hein de Vries

Prof. Dr. Catherine Bolman

Prof. Dr. Jean Muris

\section{Beoordelingscommissie}

Prof. Dr. Stef Kremers (voorzitter)

Prof. Dr. Rik Crutzen

Prof. Dr. Lilian Lechner (Open Universiteit Heerlen)

Dr. Marcel Pieterse (Universiteit Twente)

Dr. Marc Spigt 


\section{Table of contents}

$\begin{array}{llr}\text { Chapter } 1 & \text { General introduction } & 7\end{array}$

PART ONE The potential of a referral aid in the primary care setting

Chapter 2 Do professional perspectives on evidence-based smoking cessation methods align? A Delphi study among researchers and healthcare professionals

Chapter 3 A referral aid for smoking cessation interventions in primary care: study protocol for a randomized controlled trial

Chapter 4 What went wrong? A randomized controlled trial of a process and effect evaluation of a referral aid for smoking cessation counseling in primary care

Chapter 5 How to convince more primary care professionals to adopt a valued 87 smoking cessation tool: Facilitators and barriers

\section{PART TWO Future applications and possibilities}

Chapter 6 Decision aids to facilitate decision making around behavior change in the field of health promotion: a scoping review

Chapter 7 The usability of an online tool to promote the use of evidence-based smoking cessation interventions

Chapter 8 General discussion

Appendices Impact Paragraph

Abbreviation list

References

Summary

Samenvatting

Curriculum Vitae

Publication list

Dankwoord 


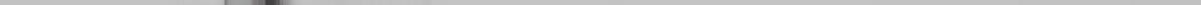


CHAPTER 1

General introduction 


\section{SMOKING (STILL) KILLS}

Tobacco has been used as a natural stimulant since it was first discovered around 5000 BCE. The first reports of the negative effects of tobacco use were published in 1602 (1) and 1761 (2); however, this message was not widely disseminated and globally accepted (though half-heartedly) until 1964 (3), and the negative effects of tobacco use were not widely accepted in the Netherlands until 1969 (4).

Today, the negative consequences of tobacco use are universally recognized, particularly the consequences of inhaling tobacco smoke through the respiratory tract (5). Yet, 1.4 billion individuals worldwide still use tobacco (6). The share of individuals who smoke tobacco differs significantly by country. Developing countries such as Nigeria have a relatively low (reported) percentage (3\%), whereas a higher share of people report smoking in countries in which inhabitants have resources to acquire tobacco products and few bestpractice smoking cessation measures have been adopted, such as Greece (31\%; (6). Tobacco use in Europe decreased starting in 2006, but the decreasing trend came to a halt between 2014 and 2017, and the average smoking percentage in 2017 was about $26 \%$ (7). In the Netherlands, the percentage of smokers declined from $60 \%$ in 1958 to $25.7 \%$ in 2014 $(6,8)$; however, the share of Dutch individuals who smoke has remained stable in recent years, indicating that a core group of smokers persists (9).

Smoking tobacco has dire consequences for society. In 2018, there were eight million deaths worldwide from cancer, cardiovascular diseases, and respiratory diseases (6); smoking is the most important and preventable risk factor for these outcomes $(6,10)$. In the Netherlands, cancer, cardiovascular disease, and respiratory disease account for approximately 20,000 deaths per year (11). In addition to mortality, smoking is associated with other societal issues, including increased health care cost, potential loss of labor caused by illness or absence, and increased inequality between high socioeconomic status (SES; $20 \%$ smoking rate) and low SES households $(30 \%$ smoking rate; $(12,13)$. Individuals in low SES groups disproportionately suffer from smoking-related diseases $(14,15)$ and are less likely than those in high SES groups to attempt to quit smoking, to seek professional help, and to successfully quit smoking $(15,16)$. Decreasing tobacco use, especially among low SES groups, is therefore an important public health focus in the Netherlands and in many other countries.

\section{THE FOCUS ON SMOKING CESSATION IN DUTCH PUBLIC HEALTH}

Several national policy measures have been implemented at the policy, organizational, and individual levels to reduce the negative public health consequences of tobacco usage in the Netherlands. 


\section{The policy level}

Policy measures aimed to reduce tobacco consumption include financial implications, constraints, and governmental campaigns. Financial implications are regular tax increases that result in an increase in the price of tobacco products $(17,18)$. Constraints that have been or will be implemented in the Netherlands include: a ban on smoking in public places such as government buildings, introduced in $2004(19,20)$ and in the hospitality sector in 2008 (21); a ban on the sale of cigarettes to people younger than 18 years, implemented in 2018 (13); a ban on the sale of cigarettes with non-tobacco flavors and non-standard colors and a ban on packaging with brand-related colors, images and logos (i.e., packaging can only contain a health warning and brand in a neutral font), implemented in 2020 (22); and a ban on stocking tobacco products in full view of shoppers (i.e., products must be stocked out of sight behind closed doors), implemented in 2021 (22). Examples of recent governmental campaigns in the Netherlands include the smoke-free generation (rookvrije generatie) campaign, which aimed to shield children from the harmful effects of (co-) smoking (23) and enable parents to raise their children in a smoke-free environment, and the Stoptober campaign, based on a UK initiative, which aimed to motivate people to quit smoking for at least 28 days in October (24). These and other policy measures have been collectively described in the Dutch National Prevention Agreement (Nationale Preventie Akkoord), which was drafted by the government and more than 70 civil society organizations in 2018. The aim of the agreement was to implement measures such as banning smoking from public spaces (e.g., playgrounds, sports clubs, and health care institutions(22).

\section{The organizational level}

Interventions at the organizational level include smoking cessation interventions specifically targeted at organizations and workspaces from different industries $(18,25,26)$. Workplace interventions are usually group-based and conveniently provided during working hours, which lowers the threshold for participation $(26,27)$. These interventions are implemented to supplement national measures and policies.

\section{The individual level}

Interventions at the individual level may be targeted directly at individuals (28-30) or delivered through health care institutions, such as primary care providers (PCPs; $(31,32)$, midwives (33), nurses working in coronary wards $(34,35)$, and other health care professionals outside of primary care (36). Of all health care providers, dentists are least likely to discuss smoking cessation with their patients (37). Obstetricians discuss smoking cessation with their patients most often, followed by medical specialists and general practitioners (37). Two-thirds of all smokers in the Netherlands access primary care services (PCS) yearly (37-39), a similar share to that of smokers in the United States (40). PCS are widely used and are often the first point of entry to professional help for people who want to quit smoking. 


\section{CHAPTER 1}

\section{The role of the primary care setting in smoking cessation}

In the 1990s, there was an increase in the general medical practice workload (41). At that time, the general practitioner (GP) was the main point of contact for smoking cessation support within the Dutch PCS. Due to concerns about ensuring the quality of primary care, reducing GP workloads, and addressing lifestyle-related issues, there was a need for additional high-quality resources for smoking cessation support referral. The function of Practice Nurses (PN; Praktijkondersteuner or $\mathrm{POH}$ in Dutch) was created in 1999 to provide chronic care support for cardiovascular risk management and lung diseases (practice nurse somatic care) and to support the treatment of mental disorders (practice nurse mental health care, POH-GGZ since 2007; (41). Smoking cessation is directly linked to chronic diseases; thus, smoking cessation support has become part of PN job responsibilities (42). Today, GPs generally provide limited smoking cessation advice grounded in the evidencebased Minimal Intervention Smoking Cessation Strategy (MIS; $(43,44)$. This smoking cessation method has been adapted for certain risk groups (45) and translated for other medical professionals, such as midwives $(46,47)$ and cardiology nurses $(48,49)$. The MIS strategy has also been applied as part of a blended care variant that combines face-to-face counseling and Web-based intervention $(28,50)$. In 2005 , about $50 \%$ of Dutch GPs used the MIS strategy at least once for smoking cessation counseling (51).

In 2009, The Dutch Guideline for Smoking Cessation Care (DGSCC) was established to assist PNs and other PCPs in the PCS to guide motivated patients to quit smoking $(52,53)$. The DGSCC, like the STIMEDIC (54) and MIS (43), shares similarities with the internationally known " 5 As" (Ask, Advise, Assess, Assist, and Arrange) method of smoking cessation (55). The DGSCC uses a stepwise approach that is comprised of the following steps: 1) provide brief quitting advice, 2) assess smoking profile, 3a) assess motivation, 3b) increase motivation, 4a) explore barriers, 4b) discuss/remove barriers, 5) discuss cessation aids, 6) help set a quit date and develop a quit plan, and 7) offer support after the quit date (52). Figure 1 presents a comparison of the 5 As method and the DGSCC method.

Adherence to all steps of the DGSCC has proven to increase patients' quit rates more successfully than providing brief quitting advice or less intensive counseling $(56,57)$. However, researchers have found that not all PNs adhere to the steps of the DGSCC (54, 58-60). Step 5-discussing evidence-based smoking cessation interventions (EBSCls)-is the step that is most often skipped by international PCPs $(58,59)$ and Dutch PNs $(31)$.

A possible reason for failing to discuss EBSCls could be practitioners' lack of familiarity with EBSCls outside the PCS. Lack of knowledge about the effectiveness of EBSCls could also be a barrier to discussing these interventions with patients (54). Other factors that have been identified as barriers to the provision of smoking cessation advice include PCPs' lack of time, not having a reason to discuss smoking behavior or having discussed the topic before, and the belief that medication is unnecessary because motivation and willpower should be enough for patients to quit (61). 


\section{As of Smoking Cessation}

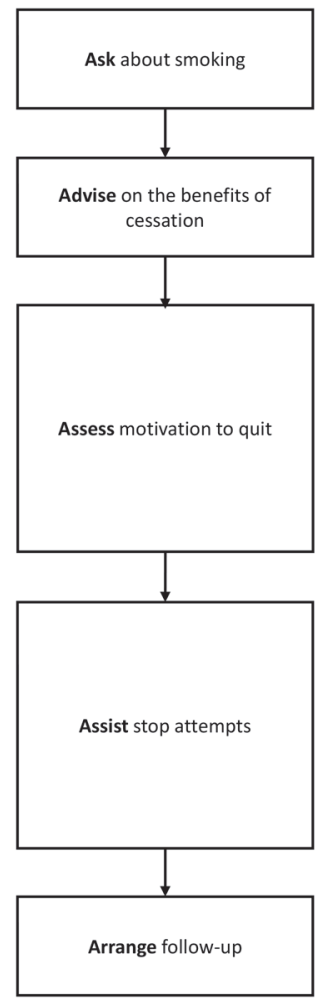

Dutch Guideline for Smoking Cessation Care

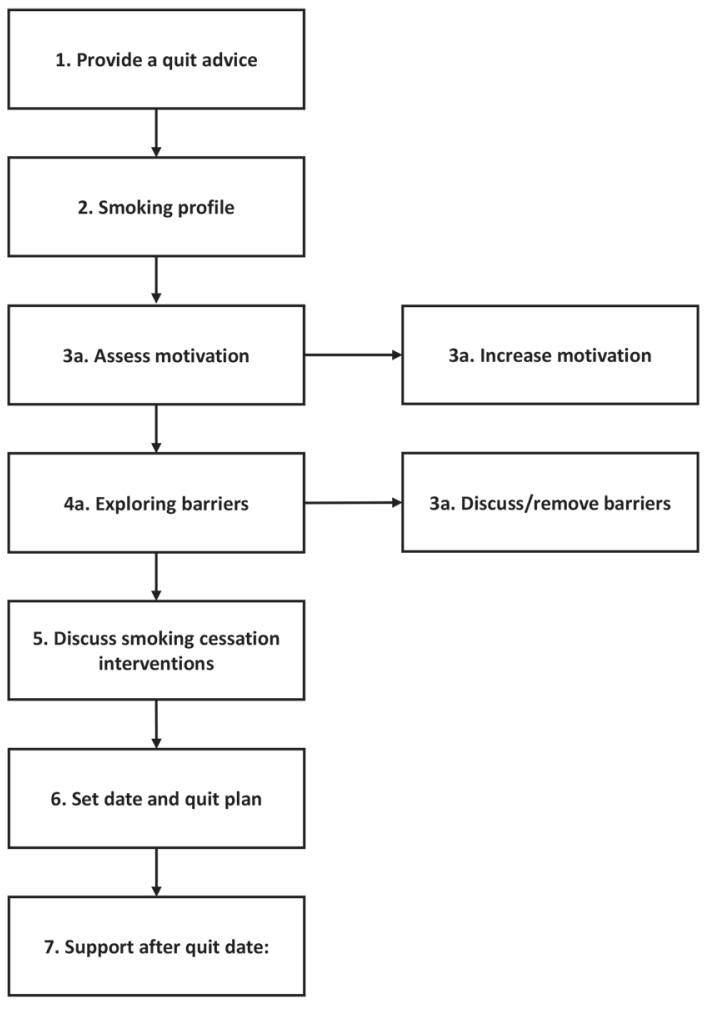

Figure 1. Schematic representation of the 5 As of smoking cessation and the DGSCC.

\section{THE OFFER AND UPTAKE OF EVIDENCE-BASED SMOKING CESSATION}

\section{AIDS}

A distinction is drawn between three types of smoking cessation aids: 1) behavioral counseling, 2) (pharmacological) supplementations and 3) non-evidence-based cessation assistance.

\section{Behavioral counseling}

Behavioral counseling uses techniques from cognitive behavioral therapy to change the thoughts, addictive responses, and habits an individual has acquired to maintain their smoking behavior (55). Behavioral counseling can consist of face-to-face counseling, eHealth, telephone counseling, and group counseling. 


\section{CHAPTER 1}

Face-to-face counseling ranges from brief quitting advice provided by a GP (which is $1-3 \%$ more effective than an unassisted quit attempt; (57) to more extensive counseling that includes at least one follow-up contact by a PN or trained stop-coach outside the PCS. The effectiveness of more extensive counseling methods varies based on the setting and the individual PCP $(44,57,62-66)$.

eHealth counseling primarily consists of tailored online counseling interventions. The effectiveness of eHealth interventions varies based on the level of tailoring to the individual's needs and the number of interactive elements. Some interventions have proven effective in comparison to a control group, including Smoke Alert (2.0; (67), Stay Quit For You (SQ4U; (68), Personal Advice in Stopping Smoking (PAS; (28), and Support to Quit (STQ; (69), all of which are based on the I-Change model described later in this chapter (70). Additional Dutch eHealth interventions are available; however, limited effectiveness data exist for these interventions due to a lack of effectiveness studies $(70,71)$.

Telephone counseling methods are similar to face-to-face counseling; however, these interventions are provided over the telephone without face-to-face contact. The effectiveness of telephone counseling ranges from $7-10 \%$ for helplines to $11-14 \%$ for counseling conducted by PCPs (72).

Group counseling is also based on behavioral techniques, but these interventions offer the added value of mutual support among participants. The effectiveness of group counseling is comparable to individual counseling of similar intensity $(73,74)$.

\section{Pharmacotherapy for smoking cessation}

Pharmacotherapy focuses on counteracting the biological aspects of nicotine addiction. Specific methods may include nicotine replacement therapy (NRT) or drugs like bupropion or varenicline (75-77). NRT consists of using nicotine gum or patches, which are available over the counter at pharmacies, drugstores, and major supermarkets (78). The use of NRT in combination with behavioral care can increase the chances of successfully quitting smoking by $50-60 \%$ in comparison to a placebo or no treatment. Higher success rates have been observed when two forms of NRT are combined (79). The success rates for pharmacotherapy range from $52-77 \%(80,81)$, and varenicline is the most effective and most often prescribed medication $(81,82)$. In the Netherlands, pharmacotherapy can only be prescribed by a GP to patients who indicate that they want to use it (52). A combination method that includes a behavioral intervention is recommended to patients who use smoking cessation medications (52), because such methods have proven significantly more effective than the use of medication alone $(81,83,84)$.

\section{Non-evidence-based cessation assistance}

Non-evidence-based smoking cessation aids are forms of therapy for which a solid evidence base has not yet been established. The most common forms of non-evidence-based cessation assistance are acupuncture or acupressure, laser therapy, and electrostimulation (85). 


\section{Reported use of EBSCls}

The use of an EBSCI has proven to double the likelihood of successful smoking cessation after 12 months $(86,87)$. However, only $25-30 \%$ of smokers who attempt to quit report using a behavioral counseling method (88-90). The range of smokers who use non-evidencebased cessation assistance is slightly lower than 25-30\% (91), and this share decreased slightly from 2012-2017 (88). Smokers may choose to use less effective cessation methods because they are not aware of the full range of available EBSCIs or are uncertain or uninformed about the effectiveness of these methods (92). Since January 2011, Dutch smokers have been allowed to claim one fully reimbursed EBSCI annually through their PCS (93); however, that claim was counted against their deductible until January $2020(22,94$, 95). Smokers may be unaware of the recent change in the rules regarding compensation for the use of EBSCls (92).

As previously mentioned, nearly $70 \%$ of smokers in the Netherlands visit their GP annually, but smoking cessation is only discussed with one-quarter to one-third of these smokers (37). In 2016 , only $37.4 \%$ of smokers who received smoking cessation advice from a PCP were advised to use an EBSCI (96). Smokers may be reluctant to use EBSCls because they have low expectations of the effectiveness of these methods $(97,98)$, they consider EBSCls unnecessary because they believe that smoking is not a problem or that they should be able to quit without support (99), or they lack knowledge of the available options (100). Data regarding the current use of EBSCls are scarce because the use of these methods is not always reported. However, $18 \%$ percent of former smokers reported using professional counseling, nicotine replacements, or medications for smoking cessation in $2018(101,102)$. Fewer than $14 \%$ of smokers reported using a method that had not been proven effective, and more than $68 \%$ reported that they did not use any cessation support $(101,102)$.

By advising smokers to use EBSCls and actively engaging smokers in choosing a cessation method that is aligned with their own expectations and preferences, PCPs can increase their patients' commitment to smoking cessation $(103,104)$. A referral aid (RA) was developed in 2019 for the purpose of this study. The RA aimed to help PNs and smokers identify an individual patient's preferred method for quitting smoking, and the aim of developing this resource was to increase the use of EBSCls for smoking cessation.

\section{INTRODUCTION OF THE STOPWIJZER INTERVENTION}

The newly developed referral aid is named "StopWijzer," which translates as "stop-guide" or "stop-smarter." The content of StopWijzer is based on a needs assessment that consisted of a literature review regarding smoking cessation in the primary care setting (e.g., (31, 52, $69,105)$. Several semi-structured interviews were conducted with GPs $(n=5)$ and PNs ( $=20$ ) to inform the development of the StopWijzer (see Chapter 3), as was a Delphi study 
of referrals to EBSCls (see Chapter 2). In addition, an advisory committee was formed, comprised of a wide range of stakeholders from different fields of health care (with a particular focus on primary care) to provide input on smoking cessation and health behavior change during the conceptualization of the StopWijzer. Finally, pilot tests were conducted among PCPs and smokers to identify their needs and incorporate them into the intervention to facilitate adoption and later use.

After conceptualizing the StopWijzer, an evaluation phase was implemented to test the effectiveness of the aid when used by PCPs in the PCS. This evaluation, conducted from 2019-2020 in the Netherlands, consisted of a randomized controlled trial as described in Chapter 4. The trial explored the effects of the StopWijzer on the use of EBSCls and smoking cessation, and it also examined PCPs' willingness to adopt the StopWijzer in their daily routine.

\section{Facilitating adoption of the referral aid}

The first step of successfully disseminating a new intervention is to achieve successful adoption among end users $(106,107)$. Adoption of the StopWijzer in daily routine requires behavioral changes and routine adjustments on the part of end users to achieve the intended effect (108). Several models have been proposed to explain and predict behavior and behavioral changes, including the social cognitive theory (109), the transtheoretical model (110), the health belief model (111), and the theories of reasoned action and planned behavior $(112,113)$. These models are all integrated in the overarching l-change model (de Vries, 2017), which is used to map beliefs about health behavior. In this case, the model is used to examine PNs' intention to adopt a new behavior related to the StopWijzer intervention.

\section{Using the I-change model to explore factors related to willingness to adopt}

The I-change model has been used in the development of a wide range of health-related behavioral interventions, including smoking cessation interventions. The l-change model has also been used to explore factors that may influence individuals' intention to adopt an intervention $(32,54,114-118)$. The model assumes three phases of motivational states that are moderated by information factors and preceding factors (see Figure 2). The three phases of motivational states include pre-motivational (awareness phase), motivational (motivation phase) and post-motivational (action phase). Researchers have identified postmotivational factors that influence adoption of an intervention, including users' perception of few barriers to adoption of a new smoking cessation method (117) and users' comfort in executing the steps of an intervention (32).

Users' motivation to adopt an intervention (daily practice of the StopWijzer, in this case) is driven by three factors: 1) attitude, 2) social influences, and 3) self-efficacy. An individual's attitude is defined as one's consideration of the perceived advantages and disadvantages of the desired behavior. For example, PNs may consider the RA a useful tool to inform 


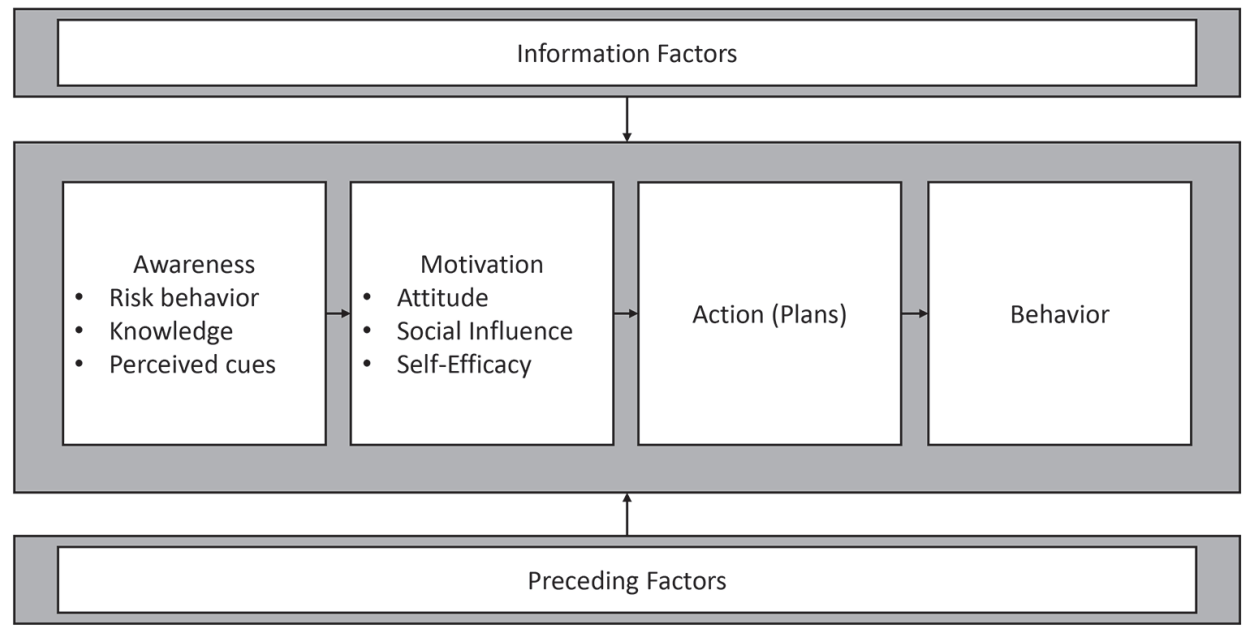

Figure 2. Simplified version of the I-Change Model, adapted from de Vries (2017) (105).

patients about smoking cessation and EBSCIs (advantage), but they may also consider the RA difficult to apply during a counseling session (disadvantage). The weighing of advantages and disadvantages results in a positive, neutral, or negative attitude toward a certain behavior. Previous research studies of the motivational factors related to the intention to adopt a smoking cessation intervention have found that attitude is a strong predictor of the intention to adopt an intervention $(32,115-117)$.

Social influence refers to users' perceptions of relevant other parties' opinions regarding the norms of a certain behavior (social norms), perceptions of pressure from other parties to perform a certain behavior (social pressure), or support from other parties to adopt a healthy behavior and perceptions of other parties' behavior (social modeling). In the case of the adoption of the StopWijzer RA, social influence refers to perceptions of colleague PCPs' opinions about and use of the RA in the PCS. Social support is less strongly associated with the intention to adopt an intervention than attitude, likely because PNs are often the only smoking cessation counseling point of contact within their individual practice (54).

Self-efficacy describes an individual's perceptions, expectations, and experiences of confidence and difficulty in performing a desired behavior, including under difficult circumstances (e.g., if the PCP is busy or the patient is not motivated). Some studies have found an association between high self-efficacy and a high intervention adoption rate (54, 114-118); however, other studies have found no such relationship (32). The combined influences of attitude, social support, and self-efficacy determine an individual's intention to change a certain behavior.

A study of the willingness of PCPs to actively refer patients to other evidence-based cessation strategies rather than provide face-to-face counseling themselves has not yet 


\section{CHAPTER 1}

been conducted. However, such a study could be valuable because PNs may be more willing or able than PCPs to apply the RA. To investigate the feasibility of the RA for adoption in daily practice, promoting and hindering factors for adoption of the intervention among PCPS in the Dutch PCS were analyzed (see Chapter 5). Prior research has indicated that PCPS sometimes lack the skill and time to assist patients with smoking cessation, and they are not often reimbursed for such activities $(119,120)$; therefore, potential applications of the RA outside of the PCS were also investigated.

\section{ALTERNATIVE APPLICATIONS}

It is challenging to reach smokers, motivate them to quit, and educate them about EBSCIs. Additionally, smokers may find it difficult to make decisions regarding the different EBSCI options $(121,122)$ due to a lack of understanding of the available options and their effectiveness. Therefore, smokers may profit from a PCP's knowledge, skills, and support in smoking cessation. To facilitate the decision process among smokers who are ready to quit, shared decision-making could be applied. In a shared decision-making process, at least two parties (e.g., the smoker and the PCP) jointly take active steps to reach a decision that they both support and agree on (123). The process of shared decision-making often requires multiple steps, including providing clear information, answering patient questions, sharing and discussing the decision (which requires a strong and open patient-caregiver bond) and, ultimately, reaching agreement between both parties (124). Adequately applying shared decision-making principles requires a significant amount of time and communication skills, neither of which are often readily available to PCPs (125).

Another way to inform smokers about their EBSCl options without requiring intensive PCP guidance is to engage patients in an informed decision-making process in which the decision-making responsibility rests entirely with the patient (126). In an informed decisionmaking process, individuals must obtain information about all relevant details of the EBSCls, including cost and effectiveness, and they must weigh the advantages and disadvantages based on their own personal priorities (127). The information can be provided in different ways (e.g., verbal, textual, or visual), provided that the information is clear, easy to understand, and unbiased (e.g., all aspects of the situation are explained). However, making a decision can still be a difficult or stressful process even with sufficient information and skills, because the smoker must assess which options best fit their values and preferences (128).

A decision aid can be deployed to support smokers in their individual decision-making processes. Decision aids are intended to aid the informed decision-making process by providing the user with all relevant information. Such aids often include value clarification exercises or methods that are aimed to help the user evaluate a wide range of options in their own specific context to determine which option best fits their needs $(127,129,130)$. 
Decision aids are often deployed to support patients in making clinical decisions in which the options radically differ (e.g., in terms of cost, effectiveness, or side effects; (127). However, decision aids have most often been used for treatment or screening decisions, and little is known about the potential effectiveness of such aids to support decision-making regarding treatment or interventions to change individual health-related behaviors (see Chapter 6).

Previous studies have found that online interventions may be particularly successful at reaching target groups of smokers who visit their PCPs less often (e.g., individuals who are young and comparatively healthy), and such interventions are also cheaper to implement and distribute than others $(131,132)$. The PCS is considered an entry point to reach smokers; however, the recruitment rate in the PCS of both PN and smoker participants for scientific research has been lower than expected $(31,132-134)$. An additional usability study was conducted among a sample of smokers recruited through the Internet to examine applicability of the RA in an (online) stand-alone form (see Chapter 7).

\section{AIM AND OUTLINE OF THE DISSERTATION}

The aim of this dissertation is to increase the use of EBSCls among smokers who are willing to undertake a quit attempt. The first part of the dissertation examines the potential of a referral aid intended to increase the use of smoking cessation interventions for smoking patients within the PCS. The second part of the thesis explores potential future applications of this referral aid outside of the PCS in the form of a decision aid.

\section{Part I: The potential of a referral aid in the primary care setting}

Chapter 2 presents the findings of a Delphi study that provides an overview of PCP and researchers' knowledge and viewpoints about the effectiveness and use of EBSCls in the field of smoking cessation. The objectives of this study were to obtain an overview of: 1) the most important criteria for SCI recommendations, 2) PCP and researchers' perceptions of the effectiveness of SCls, 3 ) important factors to consider when counseling different (high-risk) groups of smokers, and 4) PCP and researchers' perceptions of the use of e-cigarettes as an $\mathrm{SCl}$. This study examined the possibility of using a referral aid to promote the use of EBSCIs.

Chapter 3 describes the study protocol of the RA (de StopWijzer), including the trial design and the testing of the effectiveness and cost-effectiveness of the study.

Chapter 4 reports the evaluation results regarding the process and effectiveness of the RA among smoking patients who were recruited by PNs in the Dutch PCS. The aim of the process evaluation was to investigate: 1) the recruitment rate and reach of the participants, 2 ) the usage of the RA materials, and 3) the appreciation of the RA. The aim of the effectiveness evaluation was to examine the effects of the RA on 1) the promotion and 
usage of EBSCls and 2) the abstinence and smoking behavior of participants.

Chapter 5 provides an evaluation of factors associated with the intention to adopt an RA for EBSCls. This chapter includes an assessment of the differences between PCPs who did and did not intend to adopt the referral aid as part of their provision of smoking cessation counseling in the PCS.

\section{Part II: Future applications and possibilities}

Chapter 6 presents a scoping review of scientific and grey literature on decision aids to support behavioral decision-making (e.g., diet, physical activity, sleep, substance use, and smoking cessation). The literature review identifies existing decision-making processes and aids used in health promotion, positive behavioral effects, and areas for improvement (e.g., effective elements of intervention development).

Chapter 7 describes a free-standing usability study of the RA among smokers who were recruited through an online survey panel. This study examines the usability of the RA among a large sample of smokers to test whether an adapted version of the RA would be suitable as a stand-alone self-help decision tool. The thesis ends with a reflection on all studies conducted (see General Discussion) and a description of the implications for practice and further research (see Impact Paragraph). 
General introduction 
PART ONE 
The potential of a referral aid in the primary care setting 


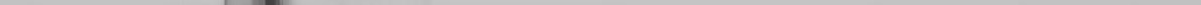




\section{CHAPTER 2}

Do professional perspectives on evidence-based smoking cessation methods align?

A Delphi study among researchers and healthcare professionals

This chapter has been published as:

Zijlstra, D. N., Hoving, C., Bolman, C. A. W., Muris, J. W. M. \& De Vries, H. (2021).

Do professional perspectives on evidence-based smoking cessation methods align? A Delphi study among researchers and healthcare professionals. Health Education Research. 


\begin{abstract}
The use of evidence-based smoking cessation interventions (SCls) can significantly increase the number of successful smoking cessation attempts. To obtain an overview of the knowledge and viewpoints on the effectiveness and use of SCls, a three-round online Delphi study was conducted among researchers and primary care professionals (PCPS). The four objectives of this study are to gain an overview of 1) the criteria important for recommending SCls, 2) the perceptions of both groups on the effectiveness of SCls, 3) the factors to consider when counseling different (high-risk) groups of smokers and 4) the perceptions of both groups on the use of e-cigarettes as an $\mathrm{SCl}$. We found a high level of agreement within groups on which smoker characteristics should be considered when recommending an SCI to smokers. We also found that PCPs display a lower degree of consensus on the effectiveness of SCls. Both groups see value in the use of special protocols for different (high-risk) groups of patients, but the two groups did not reach consensus on the use of e-cigarettes as a means to quit. Making an inventory of PCPs' needs regarding SCls and their usage may provide insight into how to facilitate a better uptake in the primary care setting.
\end{abstract}




\section{BACKGROUND}

Globally, smoking continues to be a leading cause of preventable morbidity and premature death (135). Although in the Netherlands the prevalence of smoking has decreased, $21.7 \%$ of adults (3.0 million people) still smoked in 2019. (136). Each year, around one-third of Dutch smokers report making at least one serious (at least 24 hours of no smoking) quit attempt (136). However, only a small percentage of smokers manages to quit long-term $(137,138)$.

Each year, around two-thirds of Dutch smokers visit their primary care practice (37). As such, Dutch primary care providers (PCPs) are well-positioned to initiate smoking cessation. In practice, the provision of smoking cessation support in Dutch primary care practices has to a large extent shifted from general practitioners (GPs) to trained practice nurses (PNs) $(42,114)$, who counsel and treat patients on an independent basis but operate under the responsibility of a GP (139) and rely to a large extent on the use of evidence-based guidelines and protocols (139).

In the Netherlands, the Dutch College of General Practitioners (Nederlands Huisartsen Genootschap), is responsible for developing national smoking cessation guidelines for the primary care setting (53), similar to for example the ' 5 As'(Ask, Advise, Assess, Assist and Arrange) (55).

Although a majority of PNs reports using evidence-based guidelines to structure their smoking cessation counseling (SCC) (114), important counseling elements such as increasing patients' motivation and removing cessation barriers are not always implemented $(37,61,114,140)$ PNs list several psychological (e.g., low self-efficacy to increase patient motivation) and practical barriers (e.g., difficulties in providing patients with relevant and up-to-date information) which prevent them from (fully) adhering to the guidelines (114). Another study found several barriers, such as SCC being too time-consuming and insufficient reimbursements for treating smoking patients without smoking-related illnesses (61). Similar and other barriers have been found in the primary care settings in other countries $(120,141)$.

These guidelines can be used by PCPs to structure consultations with smoking patients and discuss a range of smoking cessation interventions (SCls), focusing not only on behavioral counseling by PCPs (e.g., face-to-face counseling), but also discussing behavioral counseling outside of the primary care setting (e.g., eHealth or counseling in groups) and pharmacological interventions (e.g., nicotine replacement therapy; NTR) (86) Although a wide range of SCls with a strong evidence base is available (142), these interventions often remain underused (12). The use of evidence-based interventions to support smoking cessation can significantly increase the success rate of quit attempts (86).

This study was conducted as part of a needs assessment in the development of a referral aid which aims to support PCPs in their referral of smoking patients to SCls. To this end, we were interested in exploring the knowledge and viewpoints on the effectiveness and use of SCls among PCPs. , To our knowledge, no prior research has been conducted 
on these topics. Therefore, the first objective of this study is to gain an overview of criteria (both patient and intervention related) that are perceived to be important to consider when recommending an $\mathrm{SCl}$ to individual smoking patients. The second objective is to gain an overview of perceptions on the effectiveness of existing SCls. As smokers who visit PCPs often display smoking-related complaints such as asthma or COPD, the third objective is to gain an overview of criteria that are important to consider when counseling different (high-risk) groups of smoking patients. Other high-risk groups of smokers, as also indicated in the Dutch guidelines for smoking cessation, are pregnant smokers, smokers with a low social economic status (low-SES) and smokers with a low motivation to quit. The fourth and last objective pertains to the use of e-cigarettes. As e-cigarettes have not been proven to be an effective and safe smoking cessation aid, their use as an $\mathrm{SCl}$ is discouraged by the Dutch smoking cessation guidelines. However, to our knowledge, no research has been done on PCPs adherence to this recommendation and therefore our last objective is to gain an overview of perceptions on the use of e-cigarettes as a means to quit.

As smoking cessation is a complex health issue which is not only tackled by the primary care setting, we decided to include not only PCPs but also researchers from the field of tobacco control. Other similar structured studies have suggested that researchers are sometimes more able to identify overarching themes and bring a unique perspective $(143,144)$. Studying the perceptions of researchers and PCPs simultaneously may give a unique insight into the challenges of the current daily practice, and provide solutions to eliminate certain barriers, while also allowing us to pinpoint potential evidence-practice gaps (145). For this purpose, we used a three-round Delphi study to identify topics that are related to SCls and on which of those consensus does or does not exist among two different sets of experts.

\section{METHODS}

\subsection{Study Design}

The Delphi method is a technique for structuring communication in order to derive consensus on certain subjects for which scientific evidence is limited or conflicting by involving a panel of knowledgeable experts or individuals $(146,147)$. The three rounds of this Delphi study were conducted in the period from October 2017 to April 2018. Participants who completed both the second and third round were awarded a $€ 20$ gift voucher.

\subsubsection{First round}

Through a database search in PsycINFO, PubMed and Google Scholar, 63 researchers (national and international) were identified who had (co-)authored at least five papers on a topic related to smoking cessation in the field of health promotion, behavior change and/ or addiction in the previous five years (convenience sampling). 
The Dutch Healthcare Chart (www.zorgkaartnederland.nl), a review site of Dutch care providers and care facilities, was used to identify 21 PCPs (both GPs and PNs) who were employed in a primary care practice at the time of the study and who regularly (i.e., at least once a week) offered smoking cessation advice and counseling (i.e., actively assisting patients in a quit attempt keeping with the Dutch guidelines for smoking cessation). Only providing a brief cessation advice was used as an exclusion criterion. The questionnaires for the researchers were formulated in English and the questionnaires for the PCPs were formulated in Dutch (in all three rounds). The questionnaires were otherwise identical.

The first-round questionnaire consisted of 15 open-ended questions covering five main topics (in accordance with the study's objectives). Participants were each asked to 1) list patient characteristics that should be taken into account when recommending an SCI to an individual patient (patient characteristics), 2) list criteria that should be met by an SCI when recommending an $\mathrm{SCl}$ to an individual patient in order for it to be most effective (intervention characteristics), 3) list existing SCls they perceive to be effective, 4) list factors that should be taken into account when counseling different (high-risk) groups of smoking patients and 5) to voice their opinion on the use of e-cigarettes as a means to quit.

The responses to the open-ended questions were qualitatively analyzed by two researchers, using the Framework method (148) to merge individual responses into closedended statements and questions that were used as input for the second-round questionnaire. Duplicate items were deleted, and semantically similar items were merged. Inter-rater correlation was then calculated using Cohen's Kappa $(\mathrm{K})$. This resulted in an intercoder reliability of $99 \%$ (percentage of agreement) and a $\mathrm{K}=0.71$, indicating a substantial level of agreement between both researchers (149).

\subsubsection{Second round}

All participants who had completed the first round were invited to participate in the second round. An additional 215 researchers were identified using the same strategy as used in the first round. An additional 174 PCPs were identified through the "Dutch Register for Qualified Smoking Cessation Professionals" (Kwaliteitsregister Stoppen met Roken) (DRQSCP) (150). All 409 potential participants were then invited via email to participate in the second and third round.

The second-round questionnaire consisted of 63 closed-ended statements (see appendix - Table 2), which were based on the responses to the open-ended questions from the first round. For all questions and statements, answers were given on a 7-point Likert Scale (depending on the type of item $1=$ strongly disagree to $7=$ strongly agree, $1=$ not important at all to $7=$ very important, or $1=$ not effective at all to $7=$ very effective).

For each of the 63 items, each group's level (depending on the type of item) of agreement was analyzed by calculating the median score (Mdn) and each group's level of consensus was analyzed by calculating the interquartile range (IQR). A cut-off point of a 


\section{CHAPTER 2}

median score of $\geq 6$ was used. The IQR represents the level of consensus by calculating the difference between the $25^{\text {th }}$ and $75^{\text {th }}$ percentiles, with a smaller value indicating a smaller data spread and a higher level of consensus. An IQR $\leq 1$ is considered to be indicative of good consensus on a 7-point scale (151).

\subsubsection{Third round}

All 78 participants who had completed the second round were invited to participate in the third round, using the same procedure as used in the second round.

The third-round questionnaire consisted of the items from the second round on which consensus had not yet been reached (IQR $\geq 1$ ) in the second round. For each item, the group median and IQR from the second round was presented to the participants.

For each item, the median score and IQR was calculated. The between-group consensus was analyzed using Wilcoxon signed-rank sum tests, as the data was not normally distributed.

\section{RESULTS}

Of the 84 potential participants who were approached, 20 completed the first round $(24 \%$ response rate); 10 in each group (see Figure 1 for an overview of the recruitment process and the response rates). In the first round, the PCPs group consisted solely of PNs, who provided on average 8.2 hours of smoking cessation advice and counseling each month. The researchers group varied in experience level, ranging from junior researchers to full professors (See Table 1).

In the first round, the participants identified 12 patient characteristics (e.g., the patient's preference for an $\mathrm{SCl}$ ) and 13 intervention characteristics (e.g., the intervention continues over a longer period of time) which they deemed important when recommending an SCI to individual patients. They also listed 13 different smoking cessation interventions and 22 factors that should be taken into account when counseling four different (high-risk) groups of patients. Lastly, three different statements could be derived from participants' opinions on the use of e-cigarettes as a means to quit (e.g., e-cigarettes can be recommended as a smoking cessation intervention but not as the most preferred option).

These 63 items identified in the first round were converted into closed-ended statements and statements and included in the second-round questionnaire. In the second round, 27 researchers and 51 PCPs (both GPs and PNs) participated. The researchers group then reached consensus (IQR $\leq 1)$ on $37(59 \%)$ items and the PCPs group reached consensus on $45(71 \%)$ items (all items are listed in appendix - Table 2).

In the third round, 20 researchers and 35 PCPs (GPs and PNs) participated. Consensus was then reached by the researchers' group on 55 (87\%) items and by the PCPs group on 
$53(84 \%)$ items. Finally, between-group consensus ( $\mathrm{sig} \geq 0.05)$ was reached on $39(62 \%)$ items. The results of the Wilcoxon signed-rank sum tests can be found in appendix - Table 3. Detailed results per topic are discussed below.

Table 1. Characteristics of the participants

\begin{tabular}{|c|c|c|c|}
\hline & Round 1 & Round 2 & Round 3 \\
\hline \multicolumn{4}{|l|}{ Current occupation of health professionals } \\
\hline General practitioner (\%) & $0(0.0)$ & $23(45.1)$ & $14(40.0)$ \\
\hline Practice nurse (\%) & $10(100.0)$ & $28(54.9)$ & $21(60.0)$ \\
\hline Mean monthly hours of providing smoking cessation advice (SD) & $8.2(3.1)$ & $5.6(8.2)$ & $4.7(4.5)$ \\
\hline \multicolumn{4}{|l|}{ Current occupation of researchers } \\
\hline Junior researchers (\%) & $6(60.0)$ & $13(48.1)$ & $6(30.0)$ \\
\hline Assistant professor (\%) & $3(30.0)$ & $4(14.8)$ & $5(25.0)$ \\
\hline Associate professor (\%) & $0(0.0)$ & $6(22.2)$ & $4(20.0)$ \\
\hline Full professor (\%) & $1(10.0)$ & $4(14.8)$ & $5(25.0)$ \\
\hline \multicolumn{4}{|l|}{ Field of expertise of researchers* } \\
\hline Smoking behavior and tobacco use & 3 & $21(77.8)$ & $16(79.2)$ \\
\hline (Development of) Tobacco interventions & 7 & $14(51.9)$ & $13(61.9)$ \\
\hline (Development of) Health promotion interventions & 3 & $11(40.7)$ & $11(52.4)$ \\
\hline Other, but relevant for this study** & 1 & $5(18.5)$ & $0(0.0)$ \\
\hline
\end{tabular}

* Researchers were able to mark more than one field of expertise

** E.g., reaching low socioeconomic status groups, lung cancer screening trials, development of tobacco control policy and guideline development

\subsection{Patient characteristics that should be taken into account when choosing recommending an SCI (patient characteristics}

The researchers group reached consensus $(\mathrm{IQR} \leq 1)$ on the level of importance of all twelve listed characteristics in the second round. Of the twelve characteristics, six were rated as important or very important $(M d n \geq 6)$ (see appendix - Table 2). Over the course of rounds two and three, the PCPs group also reached consensus on the level of importance of all twelve items. With the exception of the patient's level of nicotine addiction, the PCPs group rated the same items as important or very important as the researchers group. Betweengroup consensus was reached on eight items ( $67 \%$ consensus), with three characteristics regarding the smoker's preference, experience with previous attempts and motivation rated as very important $(\mathrm{Mdn} \geq 6)$ (see appendix - Table 3 ). 


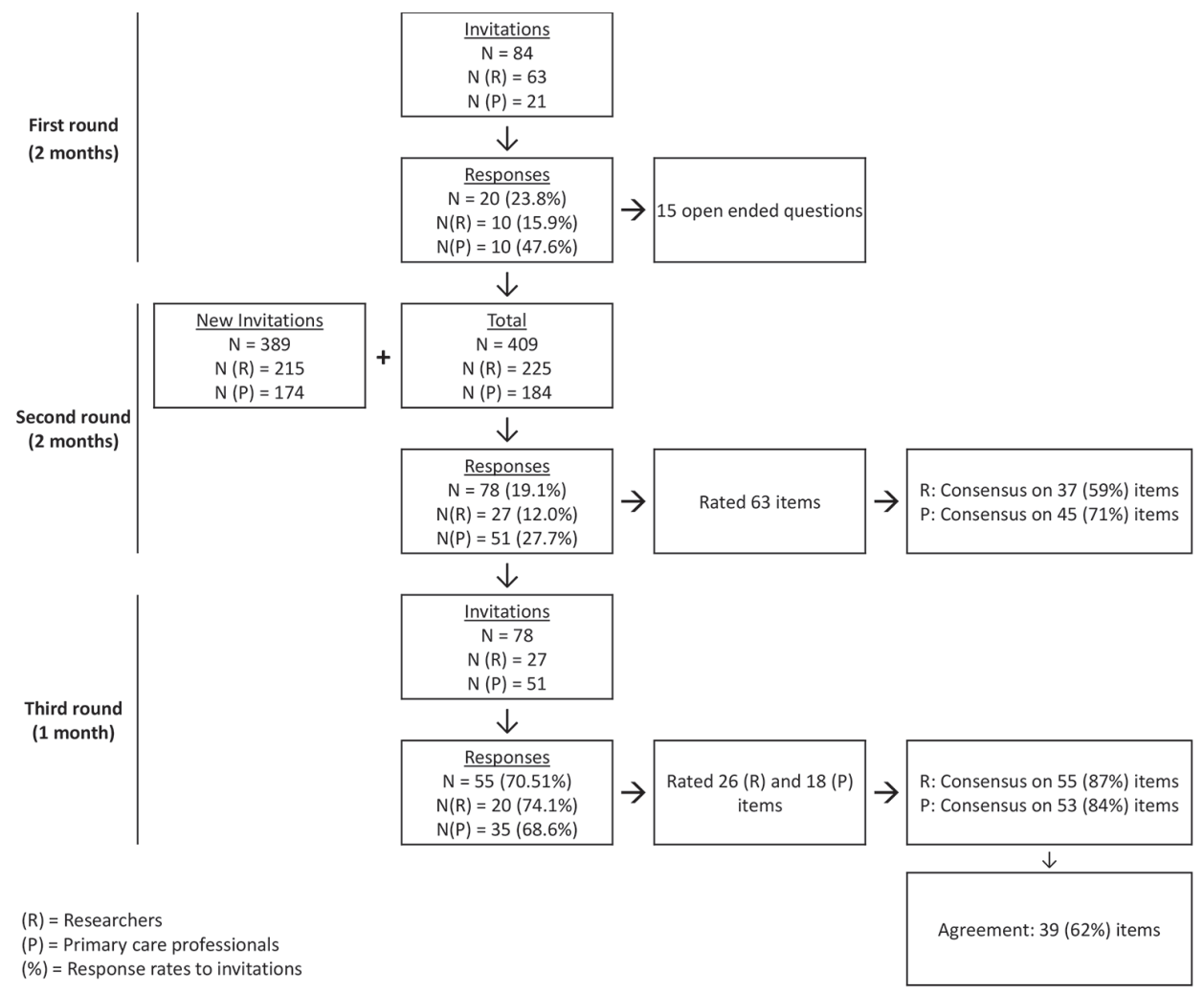

Figure 1. Overview of the recruitment process.

\subsection{Criteria that should be met by an $\mathrm{SCl}$ when choosing recommending an $\mathrm{SCl}$ for to an individual patient (intervention characteristics}

The researchers group reached consensus on the level of importance of twelve of the thirteen listed criteria over the course of rounds two and three; no consensus was reached on the inclusion of highly detailed information on smoking cessation in the intervention. Seven criteria were rated as important or very important ( $M d n \geq 6)$. The PCPs group also reached consensus on the level of importance of twelve of the thirteen items over the course of rounds two and three (all except the item regarding the length of the intervention). Four items were rated as important or very important. Between-group consensus was reached on nine items $(69 \%)$ in which high motivation scored as the most important item (Mdn = 6.5) and the intervention including fewer session as the least important (Mdn = 4.5). 


\subsection{How is the effectiveness of existing SCls perceived?}

The researchers group reached consensus on the level of effectiveness of ten of the thirteen listed SCls over the course of rounds two and three. Four of these were rated as effective or very effective (pharmacotherapy, NRT, brief cessation advice by a healthcare professional without additional interventions, and counseling in groups) ( $\mathrm{Mdn} \geq 6$ ).

The PCPs group reached consensus on the level of effectiveness of eight of the thirteen items over the course of rounds two and three. Only two items were rated as effective or very effective: (1) pharmacotherapy, and (2) brief cessation advice by a health-care professional without additional interventions. Combining the results on importance and consensus, we can conclude that consensus was only reached on the latter. Between-group consensus was reached on seven out of thirteen interventions, with pharmacotherapy scoring the highest on effectiveness $(M d n=6)$ and e-cigarettes the lowest $(\operatorname{Mdn} \leq 3)$.

\subsection{Factors that should be taken into account when counseling different (high-risk) groups of smoking patients. \\ Participants were asked to rate the level of importance of 22 factors divided over 4 (high- risk) groups of smoking patients.}

\subsubsection{Patients with smoking-related complaints:}

The researchers group reached consensus on the level of importance of five of the six listed factors over the course of rounds two and three and rated these factors as important or very important $(M d n \geq 6)$. The PCPs group reached consensus on the level of importance of all six items in round 2 and rated the same five items as important or very important as the researchers. Between-group consensus was reached on four items with the most important factors being that the smoker should be informed about his or her health risk (both Mdn = 6.5) $(67 \%$ consensus).

\subsubsection{Pregnant patients:}

Both groups reached consensus on the importance of five of the six listed factors (consensus was reached in round two); neither group reached consensus on providing pregnant patients with the same cessation support as non-pregnant patients. Except for this factor, both groups rated the other five factors as important or very important ( $\mathrm{Mdn} \geq$ 6). Between-group consensus was reached on two items with the most important factor being that the smoker should be informed of the risks of smoking during pregnancy (Mdn $\geq 6.5$ ) (33\% consensus).

\subsubsection{Patients with a low SES:}

The researchers group reached consensus on the level of importance of three of the five listed factors over the course of rounds two and three. These factors were also rated as 
important or very important $(M d n \geq 6)$. The PCPs group reached consensus on the level of importance of four of the five items over the course of rounds two and three and also rated these as important. Between-group consensus was reached on all items, with 'the smoker should be informed about his or her health risk' scoring the highest (Mdn $\geq 6.5)$ (100\% consensus).

\subsubsection{Patients with a low motivation to quit:}

Both groups reached consensus on the level of importance of four of the five listed factors (consensus was reached in round two) and rated those factors as important or very important ( $M d n \geq 6$ ). Between-group consensus was reached on four items, with the item on focusing the counseling on increasing motivation scoring the highest ( $M d n \geq 6.0)(80 \%$ consensus).

\subsection{The use of e-cigarettes as a means to quit}

The researchers group reached consensus on their level of agreement on only one of the three statements on the use of e-cigarettes as a means to quit (consensus was reached in round two): informing patients fully about the risks of e-cigarettes before talking about them as an SCl. This statement was also the only one on which agreement with the statement was reached. The group of PCPs did not reach consensus on the statements. Between-group consensus was reached on one statement, namely, recommending e-cigarettes as a means to quit but not as the most preferred option.

\section{DISCUSSION}

\subsection{Main findings}

The four objectives of this study were to gain an overview of 1) the criteria important for recommending SCls, 2) the perceptions on the effectiveness of SCls, 3) the criteria important for counseling different (high-risk) groups of smokers and 4) the perceptions on the use of e-cigarettes. These topics will be discussed below.

First, consensus within both groups was exceptionally high on the level of importance of the different criteria for recommending an SCI to individual patients. Other studies have also found that the socio-economic status and smokers' experience with previous cessation attempts play a significant role in successful smoking cessation $(152,153)$. It is also known that raising the smokers motivation to quit, for example through the use of motivational interviewing techniques, during SCC can facilitate successful smoking cessation $(154,155)$. The Dutch guidelines (53) also advise PCPs to discuss previous quit attempts and to inquire after the reasons why these failed in order to adapt treatment accordingly. 
Secondly, the PCPs group only reached consensus on the level of effectiveness of one $\mathrm{SCl}$ : a brief cessation advice by a healthcare professional without additional interventions. A brief quit advice by a health-care professional has been shown to significantly increase smoking abstinence, regardless of the patient's level of motivation (57). This corresponds with the first step of the Dutch smoking cessation guidelines. However, the combination of two or more evidence-based SCls increases the chance of a successful quit attempt $(156,157)$. The use of multiple SCls can help to increase smoking abstinence while also providing a wider range of options for smokers who are not able to visit their primary care practice or who have other preferences for SCC. The use of multiple SCIs can also take the form of in-practice counseling combined with an out-practice intervention such as eHealth or supplemental NRT.

Neither group rated the five non-evidence-based interventions (e-cigarettes, acupuncture, laser therapy, relaxation exercises and quitting without any form of cessation support) to be effective. Noted should be, however, that the level of effectiveness of three of these SCls (i.e., acupuncture, laser therapy, and quitting without any form of cessation support) was rated higher in the PCPs group than in the researcher's group. A possible explanation is that PCPs' vision on the effectiveness is based on positive (personal) experiences. Further studies should investigate whether this is the case, how this vision can be adjusted and how to best discuss non-evidence-based interventions with patients.

Thirdly, we can conclude that both groups see some value in providing additional cessation support to the four (high-risk) groups of patients. As smoking prevalence is higher among disadvantaged groups, and disadvantaged smokers may face higher exposure to tobacco's harms, they might have different needs related to cessation support $(16,117,158)$ and the necessity of providing additional support to pregnant patients is widely recognized as smoking is associated with risks for both the patient and the unborn child (159). Both groups indicated that smokers not motivated to quit should also be targeted, with the PCPs group stating that they find it important to increase motivation and use motivational interview techniques $(154,155)$.

Lastly, concerning the use of e-cigarettes, almost no consensus within or between the groups was reached. Possible explanations may be that research is still inconclusive on the use of e-cigarettes as a means to quit (160), that the quality and composition of e-cigarettes is highly variable (161), and that no concrete long-term effects in terms of effectiveness, safety and addiction are known (162-164).

\subsection{Practice implications}

First, with regards to high-risk groups of smokers, our results indicated that both groups found it important to provide additional support to high-risk groups of patients. Referring high-risk groups, such as older smokers (165) or patients with multimorbidity diseases (166), to extended cognitive behavioral therapy increases the chances of a successful quit attempt, 
so this additional support is very valuable. Yet, in practice, PCPs do not always manage to pay extra attention to these group because of a lack of self-efficacy or time $(33,54)$ or a lower motivation among these type of patients (167-169). As stated earlier, motivational interview techniques $(154,155)$ can help PCPs to increase motivation as well as convince smokers of their increased health risk (especially during pregnancy). Although the Dutch guidelines for smoking cessation mention low motivation, smoking related diseases and low-SES, specific support directions are only provided for pregnant smokers. By adding support directions for the other high-risk groups, including information on the use of SCls among high-risk groups (e.g., information on interventions designed for smokers with low language skills or interventions specifically targeted to pregnant woman), PCPs will be better able to support these groups, which may lead to higher quit percentages within these groups.

Secondly, while researchers reached consensus on the effectiveness on ten out of thirteen listed SCIs, PCPs only reached consensus on eight. In addition, as mentioned before, PCPs tend to rate the effectiveness of several non-evidence-based SCls higher than the researchers' group, which may indicate an evidence-practice gap. An evidence-practice gap sometimes is associated with a lack of knowledge or formal training in CSS (170). Despite the fact that most Dutch PCPs are registered in the DRQSCP (150), this does not necessarily prevents misconceptions about the effectiveness of SCls and this may imply the need for training programs or supplemental materials in order to fully acquaint PCPS with SCls. To our knowledge, no prior research has been conducted on the perceptions of the effectiveness of SCls among GPs and other PCPs.

Lastly, communication with PCPs on recent scientific findings may also reduce the uncertainty surrounding cigarettes, breaching the evidence-practice gap $(145,171)$. While the Dutch national smoking cessation guidelines advise against the use of e-cigarettes (172), they do not elaborate on how to approach patients who express a desire to use them as an SCl. Until a clear outcome on the use of e-cigarettes as an $\mathrm{SCl}$ is reached, a consensus on how to respond to these patients may aid PCPs in their counseling.

\subsection{Limitations}

A possible limitation is the response rate, especially among the researchers. Although these percentages are low, they are in line with those reported in similar Delphi studies or unsolicited questionnaires (173). We included international researchers to realize a large and varied sample of expertise in order to obtain a large spectrum of responses. One may argue that this could lower the generalizability to the Dutch context; yet, as the effectiveness of the SCls is very similar across countries, and international data are often used in the Netherlands in communication on the effectiveness of SCls, we believe that the level of distortion caused by this choice is probably very low.

We tried to include both GPs and PNs in the PCP group, but the first round only PNs were included. All GPs from our first round declined participation by stating that they themselves did not provide much SCC and referred us to their practice's PNs $(42,174)$. By 
recruiting PCPs via the DRQSCP (150), we successfully managed to include a more diverse range of PCPs in our second and third round. Including a wide variety of participants is a strength of the study.

It may be possible that selectivity has occurred within the group of PNs, as participants who might have a particular interest in, or a strong opinion on this topic are more likely to participate. However, when inviting PCPs we tried to include a balanced mix of occupations of PCPs who provided active SCC, to ensure a heterogenic group. This may strengthen generalizability, mainly for the national setting, but perhaps also for a part for other countries, as primary care guidelines often are similar (for example the ' $5 \mathrm{As}^{\prime}(55)$ ) even if the setting or the execution may slightly differ (e.g., SCC not being provided by specially trained PNs but by more general educated GPs)

\section{CONCLUSIONS}

This systematic exploration and consensus study focused on obtaining an overview of the knowledge and viewpoints on the effectiveness and use of SCls different smoking cessation experts. The four objectives of this study were to gain an overview of 1) the criteria important for recommending an $\mathrm{SCl}, 2$ ) the perceptions on the effectiveness of SCls, 3) the criteria important for counseling different (high-risk) groups of smokers and 4) the perceptions on the use of e-cigarettes as a means to quit. Based on a three-round Delphi-study, we found a high agreement among researchers and PCPs on which patient characteristic should be taken into account when choosing a fitting $\mathrm{SCl}$ for individual patients (e.g., taking into account the patient's needs and previous cessation attempts). We also found that PCPs display a lower degree of consensus on the effectiveness of SCls. Both researchers and PCPs see value in the use of special protocols for high-risk groups of patients, but the two groups did not reach consensus on the use of e-cigarettes as a means to quit. Making an inventory of PCPs' needs regarding SCls and their usage may provide insight into how to facilitate a better uptake in the primary care setting. 


\section{APPENDIX}

Table 2. Results per item of the second- and third-round of 'research' and 'health professionals'

Topic 1. Patient characteristics that should be taken into account when recommending an $\mathrm{SCl}$ (patient characteristics)

The smoker's preference for a specific (type of) intervention

The extent to which the characteristics of an intervention meet the smoker's needs

The smoker's background (such as educational level or health literacy skills)

The smoker's personal characteristics (such as age, gender, or overall lifestyle)

The (type of) intervention(s) the smoker has previously used

The number of quit attempts that the smoker has previously undertaken

The level of success of the smoker's previous cessation attempts

The time elapsed since the smoker's last smoking cessation attempt

The difficulties the smoker experienced in previous cessation attempts

The smoker's motivation to quit smoking

The smoker's level of nicotine addiction

The financial costs of using the intervention

Topic 2. Criteria that should be met by an $\mathrm{SCl}$ when recommending an $\mathrm{SCl}$ for an individual patient (intervention characteristics)

When the smoker is highly motivated to quit

When the intervention meets the smoker's needs as perceived by the healthcare professional

When the intervention matches the smoker's preferences

When the intervention is tailored to that what the smoker already knows about smoking cessation

When the intervention supports the smoker in developing self-control regarding smoking and smoking cessation

When the intervention includes more rather than fewer sessions.

When the intervention continues over a longer period of time

When the intervention includes highly detailed information on smoking cessation

When the intervention is used by the smoker as it is meant to be (intervention fidelity)

When an independent RCT study shows that the intervention significantly increases the likelihood of smoking cessation over 6 months or longer when compared to usual care

When an independent RCT study shows that the intervention significantly increases the number of quit attempts when compared to usual care

When the intervention is certified as being effective (for example by national smoking cessation associations)

When the intervention is recommended by national guidelines for tobacco cessation

\section{Topic 3. How is the effectiveness of existing SCls perceived?}

Pharmacotherapy

E-cigarettes 


\begin{tabular}{|c|c|c|c|}
\hline \multicolumn{4}{|c|}{ Research experts } \\
\hline Second round & & Third round & \\
\hline Mdn & IQR & Mdn & IQR \\
\hline
\end{tabular}

\begin{tabular}{|c|c|c|c|c|c|}
\hline \multicolumn{6}{|c|}{ PCPs } \\
\hline \multicolumn{3}{|c|}{ Second round } & \multicolumn{3}{|c|}{ Third round } \\
\hline $\mathbf{N}$ & Mdn & IQR & $\mathbf{N}$ & Mdn & IQR \\
\hline
\end{tabular}

$\begin{array}{lll}27 & 6.5 & \mathbf{1} \\ 27 & 6.0 & \mathbf{1} \\ 27 & 5.5 & \mathbf{1} \\ 27 & 5.5 & \mathbf{1} \\ 27 & 6.0 & \mathbf{1} \\ 27 & 5.5 & \mathbf{1} \\ 27 & 5.5 & \mathbf{1} \\ 27 & 5.0 & \mathbf{1} \\ 27 & 6.0 & \mathbf{1} \\ 27 & 6.0 & \mathbf{1} \\ 27 & 6.0 & \mathbf{1} \\ 27 & 5.5 & \mathbf{1}\end{array}$

$\begin{array}{lll}51 & 6.0 & \mathbf{1} \\ 51 & 6.0 & \mathbf{1} \\ 51 & 5.0 & \mathbf{1} \\ 51 & 5.5 & \mathbf{1} \\ 51 & 6.0 & \mathbf{1} \\ 51 & 5.5 & \mathbf{1} \\ 51 & 5.5 & \mathbf{1} \\ 51 & 5.0 & 2 \\ 51 & 6.5 & \mathbf{1} \\ 51 & 6.5 & \mathbf{1} \\ 51 & 5.5 & \mathbf{1} \\ 51 & 5.0 & 2\end{array}$

$\begin{array}{lll}27 & 6.5 & 1\end{array}$

$27 \quad 5.0 \quad 1$

$\begin{array}{lll}27 & 6.0 & 1\end{array}$

$27 \quad 5.0 \quad 2$

$\begin{array}{lll}27 & 6.0 & 1\end{array}$

$\begin{array}{lll}19 & 5.5 & 0\end{array}$

$\begin{array}{lll}50 & 6.5 & 1\end{array}$

$\begin{array}{lll}50 & 6.0 & 1\end{array}$

$\begin{array}{lll}50 & 6.0 & 0\end{array}$

$27 \quad 4.0 \quad 3$

$27 \quad 5.0 \quad 2$

$27 \quad 4.0 \quad 2$

$27 \quad 5.5 \quad 2$

$\begin{array}{lll}27 & 6.0 & 2\end{array}$

19

$\begin{array}{lll}19 & 5.0 \quad 1\end{array}$

$\begin{array}{lll}19 & 4.5 \quad 2\end{array}$

$\begin{array}{lll}19 & 6.0 & 1\end{array}$

$\begin{array}{lll}19 & 6.0 & 0\end{array}$

$\begin{array}{lll}27 & 5.0 & 2\end{array}$

$\begin{array}{lll}19 & 5.5 & 1\end{array}$

$\begin{array}{lll}27 & 6.0 & 1\end{array}$

$\begin{array}{lll}27 & 6.0 & 1\end{array}$

$\begin{array}{lll}26 & 6.0 & 2\end{array}$

$\begin{array}{lll}19 & 6.0 & 1\end{array}$

$\begin{array}{lll}19 & 3.0 & 2\end{array}$
$50 \quad 5.5 \quad 1$

$\begin{array}{lll}50 & 6.0 & 0\end{array}$

$\begin{array}{lll}50 & 4.0 & 1\end{array}$

$\begin{array}{lll}50 & 5.0 & 2\end{array}$

$50 \quad 4.5 \quad 1$

$\begin{array}{lll}50 & 5.5 & 1\end{array}$

$\begin{array}{lll}49 & 5.5 & \mathbf{1}\end{array}$

$\begin{array}{lll}49 & 5.0 & 1\end{array}$

$\begin{array}{llllll}49 & 5.0 & 2 & 32 & 5.5 & \mathbf{1}\end{array}$

$\begin{array}{lll}49 & 5.5 & \mathbf{1}\end{array}$

$\begin{array}{lll}49 & 6.0 \quad 2\end{array}$

$\begin{array}{lll}32 & 6.0 & 2\end{array}$

$49 \quad 3.0 \quad 3$ 


\section{CHAPTER 2}

Table 2. continued

Freely available nicotine replacement therapy (for example patches or chewing gum)

Acupuncture

Laser therapy

Brief cessation advice by a healthcare professional without additional interventions

Brief cessation advice by a healthcare professional in combination with pharmacotherapy

Behavioral counseling - face-to-face

Behavioral counseling - via telephone

Behavioral counseling - in groups

Behavioral counseling - eHealth

Relaxation exercises (for example mindfulness or yoga)

Quitting without any form of counseling or other resources

Topic 4. Factors that should be taken into account when counseling different (high-risk) groups of smoking patients

Smokers with smoking related complaints or conditions

The smoker should be informed about his or her health risk

The smoker should be informed about the health risks for others in their surroundings

When a smoker is motivated to quit, treatment should start as soon as possible

Counseling should be based on motivational interviewing techniques

Counseling should be tailored to the smoker's individual health problems

This group of smokers should receive the same cessation support as smokers without complaints or conditions

Smoking pregnant women

The smoker should be informed about her health risk as well as the risks for the unborn child

When a smoker is motivated to quit, treatment should start as soon as possible

The smoker's partner should be encouraged to provide cessation support

The smoker's partner should also be encouraged to quit smoking

The smoker should be informed of the risks of smoking during pregnancy

This group of smokers should receive the same cessation support as non-pregnant smokers

Smokers with a low SES

The smoker should be informed about his or her health risk

The smoker should be informed of the money they could save by quitting

Support should be focused on planning and performing alternative behaviors (coping planning)

Counseling should be based on motivational interviewing techniques

This group of smokers should receive the same cessation support as smokers with an average SES

Smokers with a low motivation to quit

Counseling should focus first on increasing motivation (quitting should be attempted after motivation has increased) 
Research experts

\begin{tabular}{lllllll}
\hline \multicolumn{2}{l}{ Second round } & & & \multicolumn{3}{l}{ Third round } \\
\cline { 1 - 3 } & Mdn & IQR & & N $\quad$ Mdn IQR
\end{tabular}

\begin{tabular}{|c|c|c|c|c|c|}
\hline $\mathbf{N}$ & Mdn & IQR & $\mathbf{N}$ & Mdn & IQR \\
\hline 26 & 6.0 & 2 & 19 & 6.0 & 1 \\
\hline
\end{tabular}

$\begin{array}{llllll}26 & 2.5 & 2 & 19 & 2.0 & 1\end{array}$

$\begin{array}{llllll}26 & 2.5 & 2 & 19 & 1.5 & 1\end{array}$

$\begin{array}{llllll}26 & 6.0 & 2 & 19 & 6.5 & 1\end{array}$

$\begin{array}{llllll}26 & 5.0 & 2 & 19 & 5.0 & 0\end{array}$

$\begin{array}{llllll}26 & 6.0 & 2 & 19 & 6.0 & 2\end{array}$

$26 \quad 5.5 \quad 1$

$\begin{array}{lll}26 & 6.0 & 1\end{array}$

$26 \quad 5.5 \quad 1$

$\begin{array}{lll}26 & 4.5 & 1\end{array}$

$\begin{array}{lll}26 & 3.0 \quad 3\end{array}$

\begin{tabular}{|c|c|c|c|c|c|}
\hline \multicolumn{6}{|c|}{ PCPs } \\
\hline \multicolumn{3}{|c|}{ Second round } & \multicolumn{3}{|c|}{ Third round } \\
\hline $\mathbf{N}$ & Mdn & IQR & $\mathbf{N}$ & Mdn & IQR \\
\hline 49 & 5.5 & 1 & & & \\
\hline 49 & 4.0 & 2 & 32 & 5.5 & 2 \\
\hline 49 & 3.5 & 1 & & & \\
\hline 49 & 6.0 & 2 & 32 & 6.0 & 1 \\
\hline 49 & 5.0 & 2 & 32 & 5.5 & 2 \\
\hline 49 & 5.0 & 2 & 32 & 5.5 & 2 \\
\hline 49 & 5.5 & 1 & & & \\
\hline 49 & 5.5 & 1 & & & \\
\hline 49 & 5.0 & 1 & & & \\
\hline 49 & 4.5 & 1 & & & \\
\hline 49 & 4.0 & 2 & 32 & 4.5 & 3 \\
\hline
\end{tabular}

$\begin{array}{ll}25 & 6.5 \\ 25 & 6.0 \\ 25 & 7.0 \\ 25 & 5.5 \\ 25 & 6.0 \\ 25 & 4.0\end{array}$

$\begin{array}{lll}25 & 7.0 & \mathbf{0} \\ 25 & 7.0 & \mathbf{0} \\ 25 & 6.5 & \mathbf{0} \\ 25 & 7.0 & \mathbf{0} \\ 25 & 7.0 & \mathbf{0} \\ 25 & 4.0 & 3\end{array}$

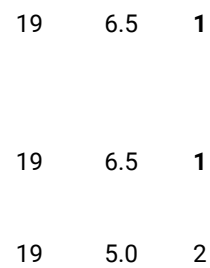

$\begin{array}{lll}48 & 6.5 & \mathbf{1} \\ 48 & 6.0 & \mathbf{0} \\ 48 & 6.0 & \mathbf{1} \\ 48 & 6.5 & \mathbf{1} \\ 48 & 6.5 & \mathbf{1} \\ 48 & 4.5 & \mathbf{1}\end{array}$

$\begin{array}{lll}25 & 6.5 & 1\end{array}$

$\begin{array}{lll}25 & 6.0 & 1\end{array}$

$\begin{array}{lll}25 & 6.0 \quad 2\end{array}$

$\begin{array}{lll}25 & 6.0 \quad 2\end{array}$

$19 \quad 6.0 \quad 1$

$19 \quad 6.0 \quad 2$

$25 \quad 4.0 \quad 3$

$\begin{array}{lll}19 & 4.5 & 3\end{array}$

$\begin{array}{lll}25 & 6.0 & 1\end{array}$

$\begin{array}{lll}48 & 6.5 & \mathbf{1} \\ 48 & 6.5 & \mathbf{1} \\ 48 & 6.5 & \mathbf{1} \\ 48 & 6.5 & \mathbf{1} \\ 48 & 6.5 & \mathbf{1} \\ 48 & 4.0 & 2\end{array}$

$\begin{array}{lll}48 & 6.5 & 1\end{array}$

$\begin{array}{llllll}48 & 6.0 & 2 & 32 & 6.0 & 1\end{array}$

$\begin{array}{llllll}48 & 6.0 & 2 & 32 & 6.0 & 0\end{array}$

$\begin{array}{lll}48 & 6.0 \quad 1\end{array}$

$48 \quad 4.5 \quad 2$

$32 \quad 4.5 \quad 3$ 


\section{CHAPTER 2}

Table 2. continued

Healthcare professionals should use motivational interview techniques

The smoker should be informed about his or her health risk

The smoker should be informed about the health risk for others in their environment

This group of smokers should receive the same cessation support as smokers who are highly motivated

\section{Topic 5. The use of e-cigarettes as a means to quit}

The healthcare provider should discourage using e-cigarettes as an aid for smoking cessation

The healthcare provider should inform the smoker fully about the use and risks of e-cigarettes before talking about them as a form of smoking cessation intervention

The caregiver can recommend e-cigarettes as an aid for smoking cessation, but not as the most preferred option 


\begin{tabular}{|c|c|c|c|c|c|c|c|c|c|c|c|}
\hline \multicolumn{6}{|c|}{ Research experts } & \multicolumn{6}{|c|}{ PCPs } \\
\hline \multicolumn{3}{|c|}{ Second round } & \multicolumn{3}{|c|}{ Third round } & \multicolumn{3}{|c|}{ Second round } & \multicolumn{3}{|c|}{ Third round } \\
\hline $\mathbf{N}$ & Mdn & IQR & $\mathbf{N}$ & Mdn & IQR & $\mathbf{N}$ & Mdn & IQR & $\mathbf{N}$ & Mdn & IQR \\
\hline 25 & 6.0 & 1 & & & & 47 & 6.5 & 1 & & & \\
\hline 25 & 6.5 & 1 & & & & 47 & 6.0 & 1 & & & \\
\hline 25 & 6.0 & 1 & & & & 47 & 6.0 & 1 & & & \\
\hline 25 & 4.5 & 4 & 19 & 5.0 & 2 & 47 & 4.5 & 2 & 32 & 5.0 & 2 \\
\hline 25 & 4.0 & 4 & 19 & 4.0 & 2 & 49 & 5.0 & 2 & 32 & 5.0 & 2 \\
\hline 25 & 6.0 & 1 & & & & 49 & 5.0 & 2 & 32 & 5.5 & 2 \\
\hline 25 & 5.5 & 2 & 19 & 5.0 & 2 & 49 & 4.0 & 3 & 32 & 4.0 & 2 \\
\hline
\end{tabular}


Table 3. Consensus between the groups of research experts and healthcare professionals

Topic 1. Patient characteristics that should be taken into account when recommending an $\mathrm{SCl}$ (patient characteristics)

The smoker's preference for a specific (type of) intervention

The extent to which the characteristics of an intervention meet the smoker's needs

The smoker's background (such as educational level or health literacy skills)

The smoker's personal characteristics (such as age, gender, or overall lifestyle)

The (type of) intervention(s) the smoker has previously used

The number of quit attempts that the smoker has previously undertaken

The level of success of the smoker's previous cessation attempts

The time elapsed since the smoker's last smoking cessation attempt

The difficulties the smoker experienced in previous cessation attempts

The smoker's motivation to quit smoking

The smoker's level of nicotine addiction

The financial costs of using the intervention

Topic 2. Criteria that should be met by an $\mathrm{SCl}$ when recommending an $\mathrm{SCl}$ for an individual patient (intervention characteristics)

When the smoker is highly motivated to quit

When the intervention meets the smoker's needs as perceived by the healthcare professional

When the intervention matches the smoker's preferences

When the intervention is tailored to that what the smoker already knows about smoking cessation

When the intervention supports the smoker in developing self-control regarding smoking and smoking cessation

When the intervention includes more rather than fewer sessions.

When the intervention continues over a longer period of time

When the intervention includes highly detailed information on smoking cessation

When the intervention is used by the smoker as it is meant to be (intervention fidelity)

When an independent RCT study shows that the intervention significantly increases the likelihood of smoking cessation over 6 months or longer when compared to usual care

When an independent RCT study shows that the intervention significantly increases the number of quit attempts when compared to usual care

When the intervention is certified as being effective (for example by national smoking cessation associations)

When the intervention is recommended by national guidelines for tobacco cessation

\section{Topic 3. How is the effectiveness of existing SCls perceived?}

Pharmacotherapy

E-cigarettes

Freely available nicotine replacement therapy (for example patches or chewing gum)

Acupuncture

Laser therapy

Brief cessation advice by a healthcare professional without additional interventions

Brief cessation advice by a healthcare professional in combination with pharmacotherapy

Behavioral counseling - face-to-face

Behavioral counseling - via telephone 


\section{N}

78

78

78

78

78

78

78

61

78

78

78

61

77

77

77

70

77

54

70

70

70

69

69

61

76

54

69

69

54

54

54

54

69

75
6.5

6.0

5.5

5.5

6.0

5.5

5.5

5.0

6.0

6.0

6.0

5.5

6.5

5.0

6.0

5.0

6.0

4.5

5.0

4.5

6.0

5.0

6.0

6.0

6.5

6.0

3.0

6.0

2.0

1.5

6.5

5.0

6.0

5.5
6.0

6.0

5.0

5.5

6.0

5.5

5.5

5.5

6.5

6.5

5.5

5.0

6.5

6.0

6.0

5.5

6.0

4.5

4.5

5.5

5.5

4.5

5.5

5.5

5.5

6.0

3.0

5.5

5.5

3.5

6.0

5.5

5.5

5.5
Wilcoxon W

.251

1841.0

1992.0

1972.0

868.5

896.0

939.5

940.0

1995.5

2009.0

1791.0

1802.0

988.0

.411

657.5

1911.0

.000

.646

925.0

.127

1886.5

.461

1044.5

.925

986.5

.459

1945.5

.958

951.5

.262

1728.0

.071

900.0

.112

1676.0

.017

1644.0

.005

1839.0

.786

912.0

.385

1717.5

.092

589.0

.000

$\mathbf{5 8 0 . 0}$

.000

1705.0

.066

1795.0

.446

1525.0

.000

1744.5

.172 


\section{CHAPTER 2}

Table 3. continued

Behavioral counseling - in groups

Behavioral counseling - eHealth

Relaxation exercises (for example mindfulness or yoga)

Quitting without any form of counseling or other resources

Topic 4. Factors that should be taken into account when counseling different (high-risk) groups of smoking patients

Smokers with smoking related complaints or conditions

The smoker should be informed about his or her health risk

The smoker should be informed about the health risks for others in their surroundings

When a smoker is motivated to quit, treatment should start as soon as possible

Counseling should be based on motivational interviewing techniques

Counseling should be tailored to the smoker's individual health problems

This group of smokers should receive the same cessation support as smokers without complaints or conditions

\section{Smoking pregnant women}

The smoker should be informed about her health risk as well as the risks for the unborn child

When a smoker is motivated to quit, treatment should start as soon as possible

The smoker's partner should be encouraged to provide cessation support

The smoker's partner should also be encouraged to quit smoking

The smoker should be informed of the risks of smoking during pregnancy

This group of smokers should receive the same cessation support as non-pregnant smokers

Smokers with a low SES

The smoker should be informed about his or her health risk

The smoker should be informed of the money they could save by quitting

Support should be focused on planning and performing alternative behaviors (coping planning)

Counseling should be based on motivational interviewing techniques

This group of smokers should receive the same cessation support as smokers with an average SES

Smokers with a low motivation to quit

Counseling should focus first on increasing motivation (quitting should be attempted after motivation has increased)

Healthcare professionals should use motivational interview techniques

The smoker should be informed about his or her health risk

The smoker should be informed about the health risk for others in their environment

This group of smokers should receive the same cessation support as smokers who are highly motivated

\section{Topic 5. The use of e-cigarettes as a means to quit}

The healthcare provider should discourage using e-cigarettes as an aid for smoking cessation

The healthcare provider should inform the smoker fully about the use and risks of e-cigarettes before talking about them as a form of smoking cessation intervention

The caregiver can recommend e-cigarettes as an aid for smoking cessation, but not as the most preferred option 


\begin{tabular}{|c|c|c|c|c|}
\hline $\mathbf{N}$ & $\begin{array}{l}\text { Mdn } \\
\text { research }\end{array}$ & $\begin{array}{l}\text { Mdn } \\
\text { PCP }\end{array}$ & Wilcoxon W & Sig. \\
\hline 75 & 6.0 & 5.5 & 1626.5 & .006 \\
\hline 60 & 5.5 & 5.0 & 1647.5 & .012 \\
\hline 75 & 4.5 & 4.5 & 1854.5 & .930 \\
\hline 54 & 3.0 & 4.0 & 753.0 & .007 \\
\hline 68 & 6.5 & 6.5 & 1659.5 & .135 \\
\hline 73 & 6.0 & 6.0 & 1633.5 & .070 \\
\hline 73 & 7.0 & 6.0 & 1414.0 & .000 \\
\hline 68 & 6.5 & 6.5 & 739.0 & .020 \\
\hline 73 & 6.0 & 6.5 & 907.0 & .818 \\
\hline 68 & 5.0 & 4.5 & 872.0 & .516 \\
\hline 73 & 7.0 & 6.5 & 1609.0 & .015 \\
\hline 73 & 7.0 & 6.5 & 1597.0 & .007 \\
\hline 73 & 6.5 & 6.5 & 1625.0 & .047 \\
\hline 73 & 7.0 & 6.5 & 1589.5 & .012 \\
\hline 73 & 7.0 & 6.5 & 1680.0 & .150 \\
\hline 54 & 3.5 & 4.0 & 1737.0 & .643 \\
\hline 73 & 6.5 & 7.0 & 1703.5 & .345 \\
\hline 59 & 6.0 & 6.0 & 1755.0 & .794 \\
\hline 54 & 6.0 & 6.0 & 1775.5 & .995 \\
\hline 68 & 6.0 & 6.0 & 779.5 & .071 \\
\hline 54 & 4.5 & 4.0 & 896.5 & .733 \\
\hline 72 & 6.0 & 6.5 & 890.0 & .768 \\
\hline 72 & 6.0 & 6.0 & 839.5 & .346 \\
\hline 72 & 6.5 & 6.0 & 1558.5 & .043 \\
\hline 72 & 6.0 & 6.0 & 1578.5 & .087 \\
\hline 54 & 5.0 & 4.5 & 1694.5 & .799 \\
\hline 54 & 4.0 & 5.0 & 760.5 & .039 \\
\hline 59 & 6.0 & 5.5 & 1573.5 & .002 \\
\hline 54 & 5.0 & 4.0 & 1704.5 & .121 \\
\hline
\end{tabular}




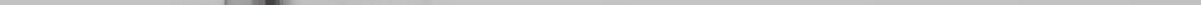




\section{CHAPTER 3}

A referral aid for smoking cessation interventions in primary care: study protocol for a randomized controlled trial

This chapter has been published as:

Zijlstra, D. N., Muris, J. W. M. , Bolman, C. A. W., Elling, J. M., Knapen, V. E.R.A., \& de Vries, $H$. (2021). A referral aid for smoking cessation interventions in primary care: study protocol for a randomized controlled trial. Primary Health Care Research \& Development, 22. 


\section{ABSTRACT}

Background: To expedite the use of evidence-based smoking cessation interventions (EBSCls) in primary care and to thereby increase the number of successful quit attempts, a referral aid was developed. This aid aims to optimize the referral to and use of EBSCls in primary care and to increase adherence to Dutch guidelines for smoking cessation.

Methods: Practice nurses (PNs) will be randomly allocated to an experimental condition or control condition and will then recruit smoking patients who show a willingness to quit smoking within 6 months. PNs allocated to the experimental condition will provide smoking cessation guidance in accordance with the referral aid. Patients from both conditions will receive questionnaires at baseline and after 6 months. Cessation effectiveness will be tested via multilevel logistic regression analyses. Multiple imputation as well as intention to treat analysis will be performed. Intervention appreciation and level of informed decision making will be compared using analysis of (co)variance. Predictors for appreciation and informed decision making will be assessed using multiple linear regression analysis and/or structural equation modeling. Finally, a cost-effectiveness study will be conducted.

Discussion: This paper describes the study design for the development and evaluation of an information and decision tool to support PNs in their guidance of smoking patients and their referral to EBSCls. The study aims to provide insight into the (cost) effectiveness of an intervention aimed at expediting the use of EBSCls in primary care. 


\section{INTRODUCTION}

Smoking remains the highest contributor to substance-attributable mortality (175). In the Netherlands, around 20.000 people die from firsthand or secondhand smoke inhalation each year (176). Consequently, in 2018, the Dutch government created the National Prevention Act which, among other things, aims to create a smoke-free generation in 2040 (22). In 2018, 36.9\% of Dutch smokers attempted to quit (101). However, only 5\% of those who quit remains abstinent after 12 months $(137,177)$.

The use of an evidence-based smoking cessation intervention (EBSCI) can double the chance of successful smoking cessation after 12 months (86). EBSCls come in two forms: behavioral counseling and supplementations. Behavioral counseling can consist of: faceto-face counseling by a health care professional (HCP), such as a general practitioner (GP) or a practice nurse (PN) or trained stop coach outside the GP-setting $(44,57,62-65)$, tailored online counseling (eHealth) $(69,178)$, telephone counseling (179) and group counseling (74). However, only $25-30 \%$ of smokers report using behavioral counseling methods (88, 89). The effectiveness of behavioral counseling can be increased through supplementation with nicotine replacement therapy such as nicotine gum or patches (available over the counter at pharmacies, drugstores and large supermarkets)(78) or pharmacotherapy (7577) of which the latter can only be prescribed in the Netherlands via the GP-setting to patients indicating they are willing to make use of them (52).

The general practice setting is a gateway to reach and advise smokers; most Dutch smokers visit their GP yearly (180) and smokers have a high level of trust in their GP (181). Theoretically, a good fit between the treatment and the patient's needs and preferences improves the patient's chances successfully quitting smoking. Discussing intervention options, their characteristics and a subsequently referral to cost-effective EBSCIs, such as eHealth interventions (70) can assist smokers in finding the best way to quit in accordance to their needs, while also lowering the time burden of smoking cessation counseling during (chronic care) consultations.

The Dutch smoking cessation guidelines for general practices instruct HCPs to actively offer smokers cessation treatment and to refer smokers to EBSCls that fit patients' needs and preferences (52). Up till ten years ago, smoking cessation support was predominantly provided by the GP. Nowadays, in most Dutch practices, smoking patients are referred to a PN (182). PNs are specialized in chronic care (42), which mostly consists of lifestyle change guidance. GPs and PNs usually use an evidence-based health counseling protocol similar to the 5-As (Ask, Advise, Assess, Assist, and Arrange) Tobacco Cessation guideline (177) as also prescribed by the Dutch smoking cessation guidelines (52) and previous studies in the GP setting $(44,183)$.

However, PNs' adherence to smoking cessation guidelines and the referral of patients to fitting EBSCls is sub-optimal $(114,184)$. This may result from PNs unfamiliarity with 


\section{CHAPTER 3}

EBSCIs, which hinders them in confidently discussing options with patients (114). Other barriers may be a high workload, a shortage of resources or an unfavorable perception of the usability of cessation guidelines $(114,185)$.

In order to expedite the use of EBSCIs in primary care and to thereby increase the number of successful quit attempts, a referral aid was developed. This aid aims to optimize the referral to and use of EBSCls in primary care and to increase adherence to Dutch guidelines for smoking cessation. This paper aims to describe the development of the referral aid, as well as the design of the associated effectiveness and cost effectiveness studies.

\section{METHOD}

\subsection{Ethical Approval}

The medical ethics committee of the University Hospital Maastricht and Maastricht University evaluated the research proposal and indicated that no medical ethical clearance for this study was needed according to the rules of the Medical Research Involving Human Subjects Act (WMO - 2018-1038). The study was registered at the Netherlands Trial Register (NL7020, https://www.trialregister.nl/trial/7020).

\subsection{Study Design}

The aim is to conduct a multi-site two-group parallel-randomized controlled trial involving an experimental condition and a control condition. Patients in the control condition will receive care as usual, which usually includes at least a mandatory brief smoking cessation advice and can be supplemented with counseling based on an evidence-based health counseling protocol similar to 5-As (Ask, Advise, Assess, Assist, and Arrange) Tobacco Cessation guideline (177) as also described in the Dutch guidelines for smoking cessation (52) and previous studies in the GP setting $(44,183)$ in the way the individual PN sees fit. In addition to the usual care, Patients in the experimental condition will receive guidance and referral by the PN in accordance with the referral aid in order to select an EBSCI that fits their needs and preferences, which acts as an expansion on the Assist and Arrange steps from the 5-A protocol (177). The chosen EBSCI can either be administered by the PN (i.e., face-to-face counseling) or coordinated by the PN (e.g., eHealth, telephone counseling).

Randomization will occur on practice level, to prevent bias between on the PN-level or the patients-level. General practices will be randomly allocated to either the control condition or the experimental condition in a 1:1 ratio. Randomization will take place via a random number generator which creates a string of numbers ( $1=$ control condition, $2=$ experimental condition) which will be allocated to general practices in order of registration. Patients are allocated based on their GP practice allocation. By allocation on practice level, PNs cannot 
accidently bias patients from the control group with information intended for the experimental group. As PNs from the experimental condition are provided with an intervention and PNs from the control condition are only asked to provide care as usual, blinding of the PNs is impossible. Patients will be semi-blinded, as they are unaware of the procedure of any other group than the one they attend.

Patients have to answer two questionnaires - at baseline and follow-up. This can be done on paper or online. Patients receive a link to the questionnaire or a paper version after they have been registered by the PN. The follow-up measurement will take place 6 months after the baseline questionnaire has been answered. The study design is illustrated in Figure 1.

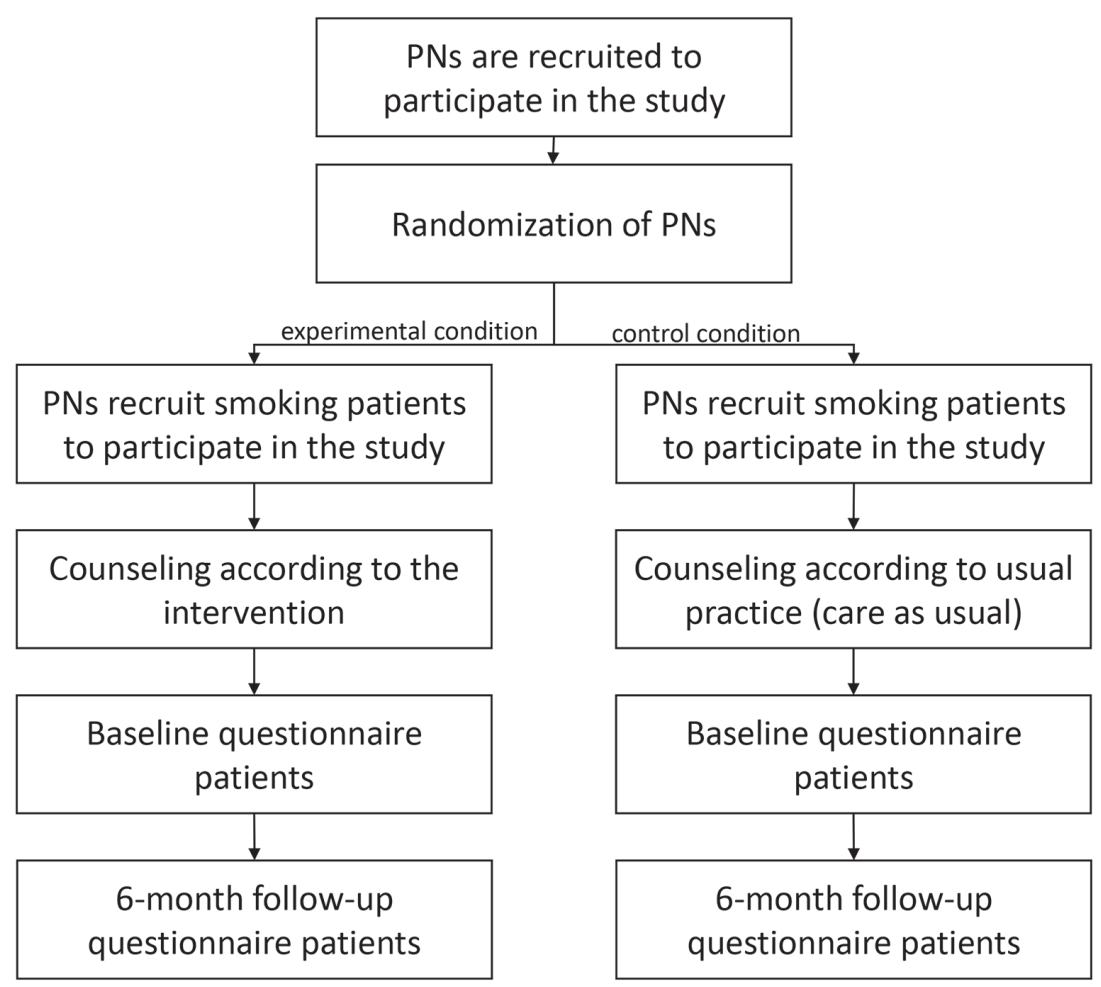

Figure 1. Flowchart of the study design.

\subsection{Recruitment of Practice Nurses}

From January 2019 until May 2020, PNs in the Netherlands will be approached to participate in the study. The task of the PNs will be twofold - recruiting smokers and referral to EBSCls in accordance with the intervention's method. 
An information package including a study invitation letter and an intervention summary will be sent to general practices spread over the Netherlands. Dutch primary care associations who support the study will also distribute the information letters to their associate general practices through their own communication channels. To gauge the interest in participating in the study, approached practices will be contacted via telephone after two weeks. Health care professionals expressing an interest in participating will be sent a more detailed guideline for the study and will be asked to sign a study participation form. PNs are requested to recruit 10 to 20 patients each. To prevent attrition and stimulate active recruitment by the $\mathrm{PNs}$, participating $\mathrm{PNs}$ who recruit at least five patients will receive a remuneration of $€ 100$. Inclusion criteria will be that PNs are employed by one or more general practices in the Netherlands and that they indicate that they provide smoking cessation counseling as such.

\subsection{Recruitment of Smokers}

From May 2019 until May 2020, participating PNs are requested to inquire about the smoking status of all their patients with smoking related complaints. Patients who report to be a smoker (no set minimum requirements), will be asked to participate in the study. If they agree to participate, they will receive brief guidance and referral advice dependent on the condition in which the PN has been assigned. Written informed consent will be obtained from all participants. Smokers will be rewarded a gift voucher of $€ 10$ for participating if they answer both questionnaires.

Inclusion criteria will be that patients smoke tobacco products, are at least 18 years old, and able to read and understand Dutch. Patients who only use e-cigarettes / e-cigars are not eligible to participate.

\subsection{Intervention: The referral aid}

The referral aid is named 'StopWijzer', which can be translated as either stop-guide or stopwiser. The content of the referral aid is based on a needs assessment consisting of a literature review (e.g. $(31,69,105))$, individual semi-structured interviews among General Practitioners (GPs) $(n=5)$, practice nurses (PNs) $(n=20)$ and smokers $(n=9)$, a Delphi study on the referral to EBSCls (not published yet) and the input of an advisory board consisting of experts representing various Dutch smoking cessation related organizations of whom six were actively involved

The PNs in the experimental condition will receive an intervention's manual to aid them in discussing smoking cessation with their patients and to help them select an EBSCI that fits the patient's needs and preferences. Smoking cessation interventions which are included in the referral aid are 1) face-to-face counseling (44), 2) counseling via internet (eHealth) $(69,178), 3)$ telephone counseling $(179), 4)$ group counseling $(74), 5)$ pharmacotherapy and 6) nicotine replacement therapy. It is strongly recommended to only offer pharmacotherapy and nicotine replacement therapy in combination with a form of behavioral counseling, as 
is also counseled by the Dutch smoking cessation guidelines (52).

The use of non-evidence-based methods such as acupuncture and the use of e-cigarettes is also discussed in the referral aid as smokers might inquire about the effectiveness of these methods. Th referral aid actively encourages PNs to not recommend these methods and stimulates them to advance the aforementioned EBSCls as suitable alternatives.

The PN starts with inquiring about the patients' smoking habits and his or her interest in undertaking a smoking cessation attempt. They also inform the patient about the referral aid and the underlying study (see also Figure 2).

Patients who agree to participate in the study will be counseled in accordance with the referral aid. Firstly, the PN inquires about earlier cessation attempts and smoking cessation methods patients may have used during these attempts. Secondly, the PN informs the patient about the available EBSCls and their advantages and disadvantages based on the information provided in the intervention's manual. If necessary, the manual also allows PNs to provide information on possible reimbursements by health insurers. Thirdly, the PN and patient discuss which EBSCI best fits the patient's needs and preferences. Fourthly, the smoker selects an EBSCI and chooses a cessation date. Lastly, depending on the EBSCI chosen by the patient, PNs schedule at least one follow-up appointment in 2-5 weeks, in order to evaluate and, if necessary, to select another EBSCI.

If the smoker is not (yet) interested in participating, PNs are advised to give the smoker a flyer to take home. This flyer contains information about participating in the study and a summary of the different EBSCls. This way, smokers may be stimulated to consider smoking cessation at a later point of time. If the smoker is uncertain about participating, PNs are advised to, in addition to handing out the aforementioned flyer, schedule a follow-up meeting or telephone call with the smoker for further discussion on participation in the study.

\subsection{Intervention materials}

Materials will consist of a small (letterbox sized) package which will be sent via post and a website (www.stopwijzer.nu). Taking into account the potential of low health literacy of patients, the materials have been written in clear and comprehensible language in accordance with the applicable Dutch guidelines (Language level B1) (186). The main component is an instruction manual for using the referral aid. This manual consists of the following elements (see also Figure 3):

1. an introduction, which explains the goals and relevance of the intervention and gives a brief overview of the different EBSCls and the other elements of the referral aid;

2. instructions in using the referral aid, which include a roadmap of the most important steps and a summary in the form of a flowchart;

3. an overview of possible reimbursements of EBSCl's by health insurers with a calculation tool to help patients provide insight into how much money they can save by quitting smoking; 
4. an overview of the different EBSCIs, in the following order: face-to-face counseling, eHealth, counseling via telephone, group counseling, nicotine replacement therapy, pharmacotherapy and non-evidence based 'cessation' methods (acupuncture (187), laser therapy (188), auriculotherapy (189), hypnoses (190) and e-cigarettes (191));

5. guidelines for following up the initial consultation;

6. some concluding remarks and room for taking notes.

\subsubsection{Flowchart handout}

Two of the elements of the instruction manual, a summary of the referral aid and a summary of health insurer's reimbursements policies, are printed on A5 carton handouts in order to be used by PNs as a quick reminder (see also Figure 4).

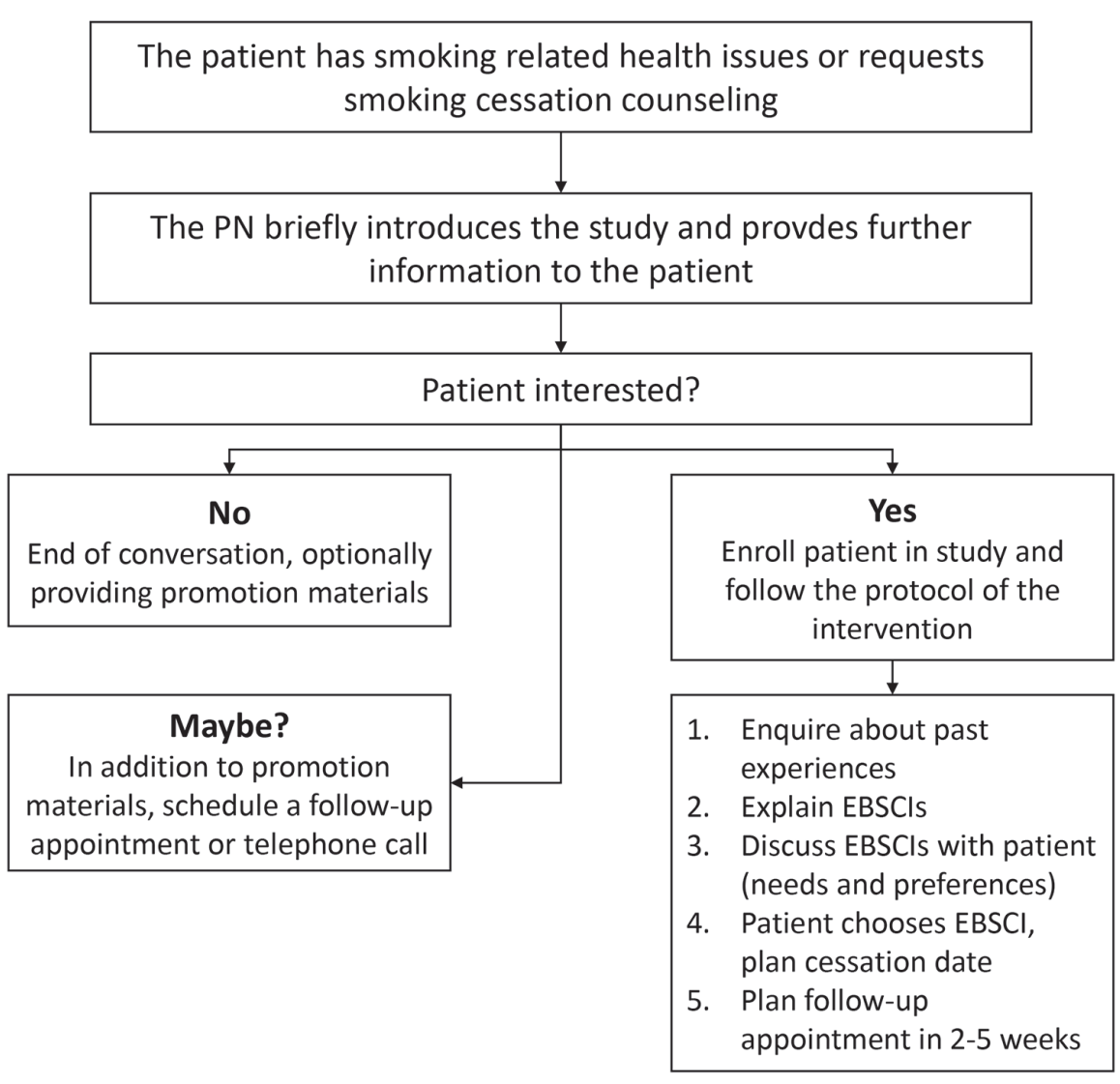

Figure 2. Flowchart referral aid 


\section{INLEIDING}

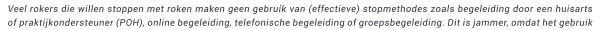

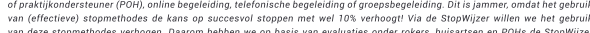
van deze sit
ontwikkeld.

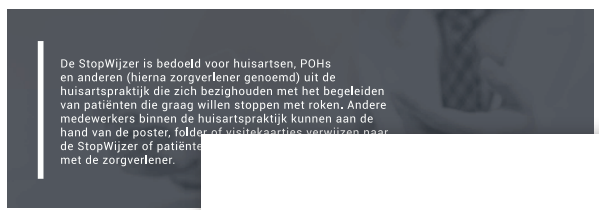

WAT IS DE STOPWIJZER

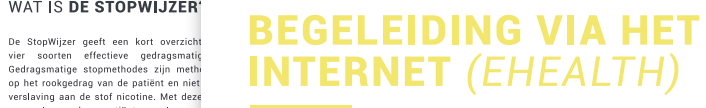

verslaving aan de stof nicotine. Met deze zorgverleners hum patienten volgens,
inzichten effectief ondersteunen bij het st De StopWijer is gemaakt op basis van een
evaluatie. De zorgvertener helpt de roke

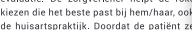
een bepaalde methode (of een combinatie deze meer gemotiverd het stoptraject
kans op en sucessolle stoppoging.

De volgende gedrags

StopW $W_{\text {ijer toegelicht: }}$
1. Stoppen door beged

persoonlijke stopcoact:

2. Stoppen door effectieve begeleiding $v$

4. Stoppen via effectieve groepsbegele

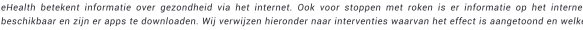
bij het 'Loket Gezond Leven' staan geregistreerd als effectief., Deze methodes maken ook vask gebruik van de stappen die bij de

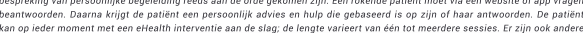

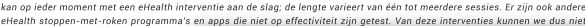
eHealth stoppen-met-roken programma's en apps die niet op effectiviteit zijn getest. van deze interventies kunnen we dus niter

UW RoL

Als de patieint middels begeleiding via het

bespreekt u samen de voor- en nadelen var
Dok bespreekt 4 war de patiënt terecht $k$

via het internet en hoe dit in zijn werk gaa
vragen zelf thuis te kijken en contact op

samen een aanbieder uitzoeken. U $\mathrm{kep}$.
vervolgatspraken plannen om met de pat de stoppoging gatt, hoe de begeleiding ge

\section{NA HET GESPREK}

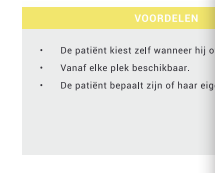

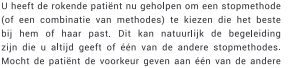

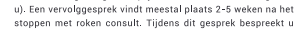
AANBiEders EFFECTIEVE TrAiningen GE

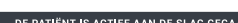
mogelijkhed
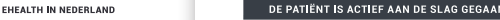

DE PATIENT HEEFT NOG GEEN ACTIE ONDERNOMEN

Stoppen met roken $2.0,1$ sessic
$\rightarrow$ http $/ /$ www. health-alert

Steun bij stoppen, minimal 3 sessies (gra

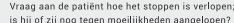

Vraag de patiënt expliciet naar zijn of haar

ervaringen met de gekozen hulpmiddelent.

Bespreek samen of de patient hierbij nog hulp

Als de patient niet op het consult is verschenen
kunt u proberen de patient via andere wemen kunt u proberen de patient via andere wegen
te bereiken f(bivoorbeeld tele foonich of email). Herinner de patiênt eraan dat jullie een

Jellinek (gratis)
$\rightarrow$ http://www.zelfhulptabak.n//Portal

Figure 3. Brief overview content interventions' manual 


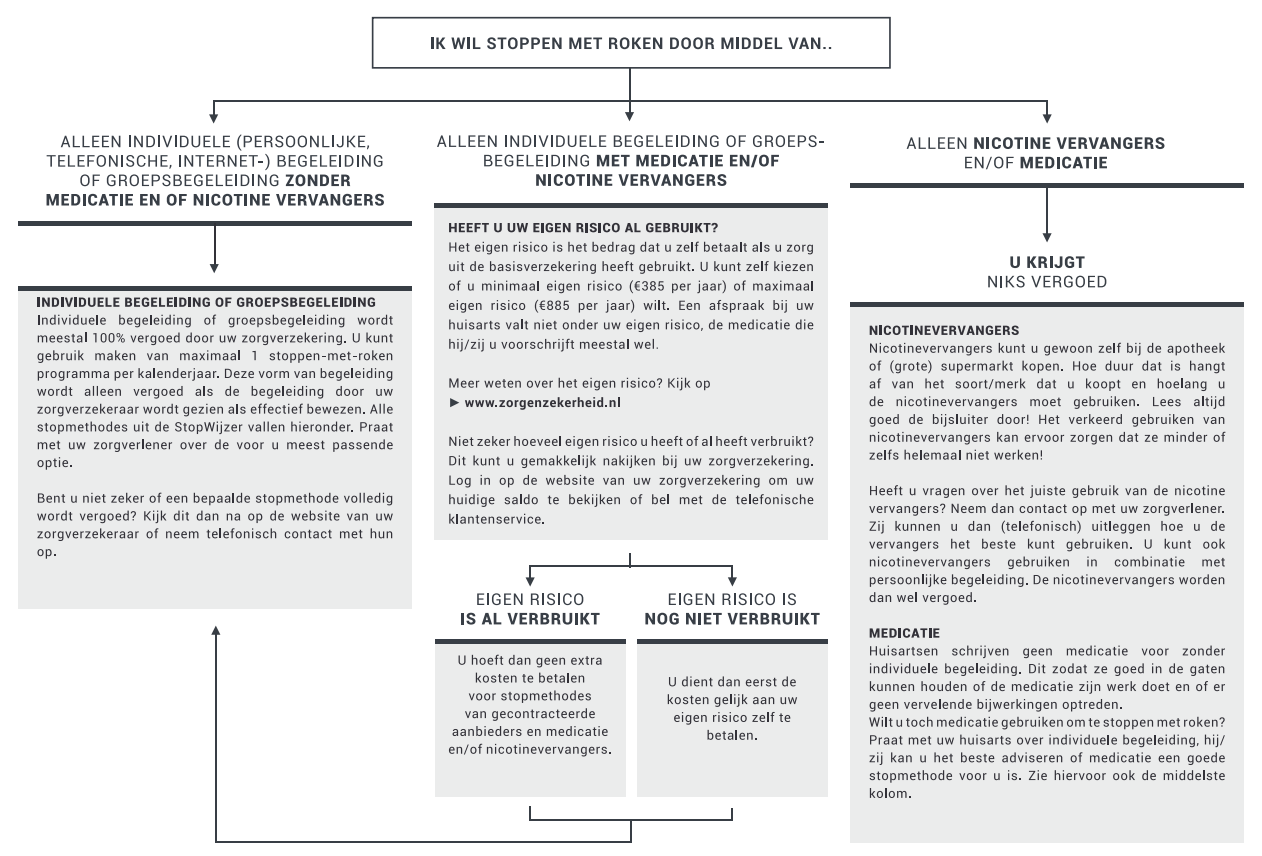

Figure 4. Flowchart handout

\subsubsection{Placemat}

PNs also receive a decision matrix printed on a plastic desk pad. The desk pad can be used as a reminder for the $\mathrm{PN}$ or as a conversation tool during a consultation with a smoking patient (see also Figure 5). The matrix lists the EBSCls mentioned above and gives an outline of their target groups, strengths and weaknesses, effectiveness, and costs. The matrix can also be accessed via the project's website.

\subsubsection{Flyers}

PNs will receive flyers on which the information on EBSCls is summarized and which list the contact information of the research team and a link to the project's website.

\subsubsection{Promotion materials}

In addition, the package also will contain a stack of business cards with the contact information of the research team and a link to the project's website. Also enclosed will be a poster which is to be used to promote the project in the practice's waiting room. A digital version of the poster which van be displayed on waiting room screens will be sent via email. Lastly, a pen and a notebook featuring the project's logo will be included as a small reminder. 


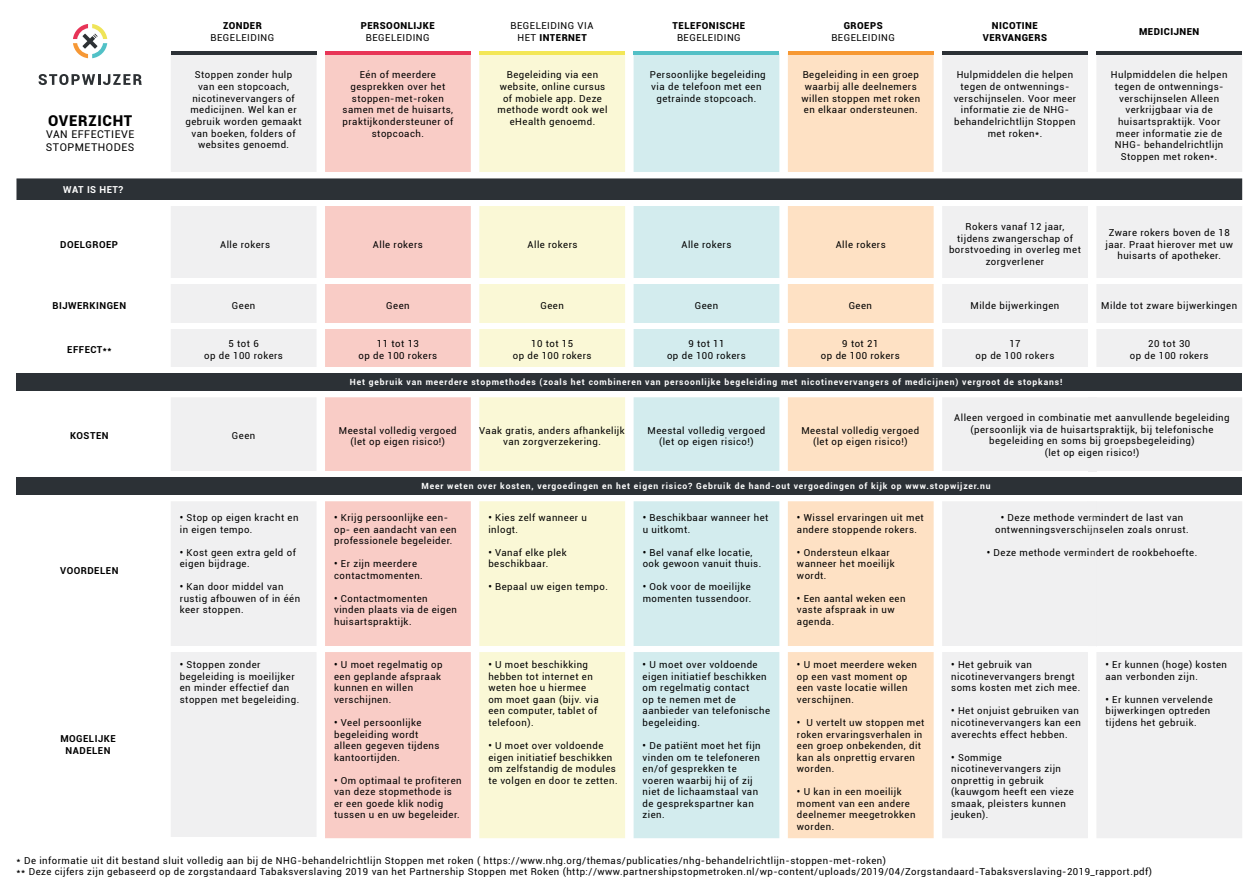

Figure 5. Decision matrix, provided in the form of a desk pad and available online.

\subsubsection{Website}

The project's website will consist of a general part and a password-protected part. PNs from both the experimental and the control condition will be able to register new patients in the password-protected part. PNs from the experimental condition can furthermore access digital copies of the manual and other materials (e.g., the placemat, handouts, and flyer). Patients from the experimental condition can access materials delivering the same information as they receive from their PN. PNs and patients form the experimental condition can access a frequently asked questions (FAQ) page tailored to their needs. Smokers from the control condition can only access the general part of the website, which will contain general information about the project and the study

\subsection{Prompts to promote intervention use and to prevent questionnaire attrition}

PNs will be sent a newsletter once a month in order to keep them informed of the progress of the study and to remind them of their participation. PNs who lag in recruitment will be approached personally via telephone call or practice visit. Further, PNs will be sent personal postcards during the holiday season or when they have recruited their fifth participant. Participants will be able to contact the research team via the project's website or via email or telephone. 


\section{CHAPTER 3}

Additionally, to the questionnaires we sent directly to the patients, we also sent out paper questionnaires to the general practices, to be delivered to patients by the PN, that intend to reduce attrition in the period between the initial meeting and receiving the questionnaire via post or email. Smokers can complete these paper questionnaires directly after their meeting with a PN, for example in the practice's waiting room. Pre-addressed envelopes including postage are provided with all paper questionnaires.

\subsection{Usability testing}

A prototype version of the intervention was tested by five HCPs in order to identify any ambiguities within the intervention. Open interviews were held in which the HCPs reviewed the materials together with the primary researcher and the HCPs' comments and opinions were used as input for the final intervention. Most changes were minor and related to terminology, for example using the term HCP instead of PN in materials to not exclude other possible participants. Other comments focused more on design elements such as making a more distinct distinction between EBSCls and non-evidence-based interventions using visual techniques.

\subsection{Data collection}

\subsubsection{Measures}

\subsubsection{Tobacco abstinence}

The primary outcome of the study will be 7-day point prevalence abstinence measured at six month follow-up from baseline $(192,193)$. Secondary outcomes are 24-hour point prevalence abstinence and 3-month prolonged abstinence (193). Assessing 6-month prolonged abstinence is not possible due to the study design, since follow-up measurement will take place 6 months after answering the baseline questionnaire irrespective of the date the participant quit smoking. Prolonged abstinence will be assessed by asking patients whether they have refrained from smoking tobacco since their last quit attempt allowing for a 2-week grace period during which the participant could smoke 1 to 5 cigarettes. Furthermore, patients will be asked whether and how often they have tried to quit smoking (i.e., no cigarette for 24 hours) during the intervention period. If patients indicate to smoke at follow-up, they will also be asked how many cigarettes on average they smoke per day. Because of minimal personal contact with the research staff and reduced possible contact with HCPs because of Covid-19, biochemical validation will not be possible. Therefore, all tobacco abstinence measures will be assessed at 6-month follow-up using self-report with an added 'bogus pipeline' question ('Do you object if we come to do a saliva test to check your smoking status?) to reduce socially desirable responses by including the threat of biochemical testing $(194,195)$. Previous studies suggest that the difference between self-reported abstinence rates and those verified with biochemical validation is negligible (196-198). The number of cigarettes smoked on average per day will be measured at baseline as well. 


\subsubsection{Smoking cessation method chosen}

Patients will be asked which EBSCls they have used in any previous smoking cessation attempts and to grade the methods they have used on a scale ranging from $1=$ very bad to 10 = very good. The following EBSCls constitute the response options: face-to-face counseling, eHealth, telephone counseling, group counseling, pharmacotherapy, and nicotine replacement therapy. If a participant uses a smoking cessation method that is not included in the response options, it will be possible to indicate this entering free text. The smoking cessation method selected will be assessed at 6-month follow-up.

\subsubsection{Quality of life and health care costs}

Quality of life measures EuroQol (199) and ICECAP (200) will be used to measure the incremental costs per quality adjusted life year (QALY). Health care costs (e.g., productivity losses, medical consumption and consumption of informal care) will be measured via the iMTA medical Consumption Questionnaire (iMCQ (201). The valuation of costs will be based on the latest Dutch standards, which include for example hourly wage of HCPs and standardized costs for consults in the GP setting $(202,203)$. Quality of life and health care costs will be assessed both at baseline and at 6-month follow-up.

\subsubsection{Informed decision making}

Decisional conflict (e.g. "I feel I have made an informed choice") will be assessed via 16 items on a 5 -point Likert scale $(1=$ strongly disagree; 5 = strongly agree) $(204,205)$. Decisional conflict will be assessed at 6-month follow-up.

\subsubsection{Contact between PN and smoker}

The contact between the PN and the smoker will be evaluated because a constructive and empathic relationship between $\mathrm{PN}$ and smoker is an important factor for intervention success (206). Firstly, patients have to indicate which topics have been discussed during the guidance and referral advice (e.g., "He/she (the PN) has asked you how motivated you are to stop smoking" ( $0=$ yes; $1=$ no). Secondly, the relationship between smoker and PN will be assessed via six items (e.g. "During a conversation about quitting smoking, I have the feeling that my caregiver is offering me choices") on a 5-point Likert scale ( 1 = strongly disagree; 5 = strongly agree). The contact between the PN and the smoker will be assessed both at baseline and at 6-month follow-up.

\subsubsection{Appreciation of the intervention}

After assessing which intervention materials have been noticed by the patients (e.g. "Have you seen the intervention's poster in the waiting room of your general practice?; 0 = yes; 1 = no), appreciation of the materials (e.g. "I think the StopWijzer materials are understandable") will be assessed via four 5-point Likert items ( 1 = strongly disagree; 5 = strongly agree). 


\section{CHAPTER 3}

Moreover, patients will be asked to grade the intervention materials ( 1 = very bad; $10=$ very good) and patients and for comments and suggestions entering free text. The appreciation of the intervention will be assessed at 6-month follow-up.

\subsubsection{Demographics, smoking characteristics, health status, and health literacy}

Demographics will be assessed via age, gender $(0=$ male; $1=$ female), nationality $(0=$ other nationality; 1 = Dutch nationality) education level ( 1 = low: no education, primary, or basic vocational school; 2 = medium: secondary vocational school or high school; 3 = high: higher vocational school or university) and occupation of the principal wage earner of the household.

Motivation to quit smoking (e.g. 193, 207) will be assessed via four items. Three items (e.g., "I am planning to quit smoking") use 7-point Likert scales ( 1 = certainly not; 7 = certainly yes). One item assesses whether smokers want to quit smoking including the time frame ( 1 = yes, within one month; 2 = yes, within three months; 3 = yes, within six months; 4 = yes, within one year; $5=$ yes, but not within one year; and $6=$ no, I do not plan to quit smoking).

The intention to use a specific smoking cessation method will be assessed via 20 items (e.g., "In order to stop smoking, I can best make use of nicotine replacement therapy"). All items use be 7-point Likert items ( 1 = certainly not to 7 = certainly yes). The questions were developed for this study based on the I-Change model, which aims at explaining motivational and behavioral change via integrating various social-cognitive theories (105).

The current use of e-cigarettes will be assessed via one item ("Do you use e-cigarettes?"; 1 = no; 2 = yes, without nicotine; 3 = yes, with nicotine).

Cigarette dependence will be assessed via the Fagerström Test for Cigarette Dependence $(208,209)$. The six items of the scale will be converted into an overall score ranging from 0 to 10 . The dependence level is classified as $0-2=$ low; $3-4=$ moderate; $5-6$ = strong; and 7-10 = very strong.

The health status of the smoker (e.g. "Do you have type 2 diabetes?") will be assessed for six diseases ( $0=$ "yes"; $1=$ "no"): COPD, cancer, type 2 diabetes, cardiovascular diseases, asthma, and depression (210).

Health literacy (e.g. "How often do you get help with reading letters or folders of your GP, the hospital or other health care services?") will be assessed via three items ( 1 = never; 5 = always (211). All variables described in this paragraph will be assessed at baseline.

\subsubsection{Sample size}

A power analysis for logistic regression was conducted using $G^{\star}$ Power version 3.1 (212). Considering an effect size (odds ratio) of 0.30 , a power of .80 , and an alpha of .05 , a total sample size of 292 patients will be required. The effect size was calculated for a $10 \%$ difference between control condition and experimental condition. Seven-day point prevalence abstinence was estimated to be $5 \%$ in the control condition and $15 \%$ in the experimental condition. 
Assuming that patients are nested within general practices with an average cluster size of five patients, a total sample size of 300 patients will be required. Based on earlier smoking cessation studies in general practices, intra cluster correlation (ICC) was set at .01 . We will aim to recruit 60 PNs that need to recruit an average of 10 patients each. Considering a dropout rate of $50 \%$, there will be five patients per GP-practice that filled out the baseline questionnaire, totaling in 300 patients. In order to account for drop out at 6 months follow-up, multiple imputation will be conducted applying Multivariate Imputation via Chained Equations (MICE) in R $(213,214)$.

\subsubsection{Data analysis}

All analyses will be performed following the intention-to-treat principle. To account for missing observations in the 6-month follow-up questionnaire, multiple imputations will be conducted applying Multivariate Imputation via Chained Equations (MICE) in R $(213,214)$.

Firstly, descriptive analyses will be conducted to describe the sample characteristics. Secondly, logistic regression will be used to analyze attrition, including baseline factors and condition as predictors. Thirdly, if sample size allows, multilevel logistic regression analyses will be performed to assess differences between conditions in 7-day point prevalence abstinence, 24-hour point prevalence abstinence, and 3-month prolonged abstinence. Fourthly, analyses of variance will be performed to test for differences in decisional conflict and appreciation of the intervention materials between conditions.

The economic evaluation will involve the performance of a combination of a costeffectiveness analysis (CEA) and a cost-utility analysis (CUA) of data collected during the baseline and 6-month follow-up measurement (see: quality of life and health care costs). In a CEA, effects are presented in clinical outcomes (here: additional quitters). In the CEA, the incremental cost-effectiveness ratio (ICER) will be expressed as the incremental costs per additional quitter (measured as 6 months Point Prevalence Abstinence (PPA)). The primary outcomes measure for the CUA will be QALYs, measured via EuroQol (199) and ICECAP (200). The economic evaluation will be performed from a health care and societal perspective implying that all relevant costs and outcomes will be considered. Intervention costs, healthcare costs, patient, and family costs (in a subsample), and costs outside the health care sector will be assessed.

\section{DISCUSSION}

\subsection{Potential strengths of the study}

The first potential strength of the study is the use of a random allocation of PNs via a computer algorithm to mitigate possible biases within general practice settings as PNs working within the same general practice setting but allocated to different conditions may 
have an impact on the implementation of the study. Secondly, the intervention was pilot tested among a group of potential users, both PNs and smokers, and experts from the field to test the usability and to remove ambiguities. Thirdly, the GP setting was used as a gateway to reach the target group as most smokers visit their general practice yearly (180) and have a high level of trust in their GP (181) which makes it more accessible than specialty centers at the hospital level. Fourthly, the intervention consists of a summary of various already proven effective smoking cessation methods, which are often underused at the moment. This study may help to increase their uptake. Fifthly, a cost-effectiveness study will be performed, which will provide an estimation of the additional costs and benefits of the intervention as compared to care as usual. Sixthly, in order to take into account, the potential of low health literacy of patients, the materials have been written in clear and comprehensible language in accordance with the applicable Dutch guidelines. Lastly, using the intervention takes hardly any additional time, making it a perfect fit in the timeslots their usual consultation sessions. This makes it easier for PNs to participate in the study.

\subsection{Potential limitations}

Firstly, practice nurses provided less smokers than expected, leading to a longer inclusion period and omission of also a 12 month follow up as stated in our trial register, (NL7020/ NTR7218). Yet, a 6 months study follow up is still an acceptable period for assessing treatment effectiveness (215).

Secondly, although we aim to include all eligible smoking patients who visit the participating general practices, there is a risk of selection bias by PNs. PNs may tend to invite more smokers who have already shown a willingness to stop smoking or who are deemed to be more easily motivated to participate, as also seen in other studies $(54,114$, 216). The conversational guidelines found in the intervention's manual and flowchart handout are aimed to induce PNs to include all types of smokers.

Thirdly, because smokers often just have one face-to-face meeting with a PN and we use mostly online questionnaires at the six months mark, we may face a high attrition rate. This is usual for studies that use online questionnaires (217-219). We tried to overcome this by sending additional questionnaire packages so that patients could answer the questionnaire on site if desired.

In order to try to prevent attrition at the follow-up assessment, we will also provide a shortened questionnaire including three of the aforementioned questions on abstinence (7-day point prevalence abstinence, 24-hour point prevalence abstinence, and 3-month prolonged abstinence) and one question asking the patients about EBSCIs used during their cessation attempt. This shortened questionnaire can be administered via email or telephone.

Lastly, the efficacy of counseling treatment is also dependent on contextual factors, such as the patient-counselor relationship (220). However, due to the nature of this study, targeting the use of evidence-based smoking cessation methods, assessing these contextual factors was beyond the scope of this study. 


\section{CONCLUSION}

This paper describes the study design for the development and evaluation of an information and decision tool to support PNs in the guidance of smoking patients and the referral to evidence-based smoking cessation interventions. The results of this study aim to provide insight into the (cost) effectiveness of an intervention aimed at promoting the use of more evidence-based smoking strategies, arriving at a more personalized referral decision and the best way to communicate them to smoking patients. The behavioral effectiveness, as well as the cost-effectiveness, will be reported on in later papers. 


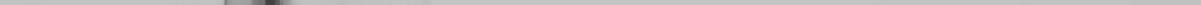




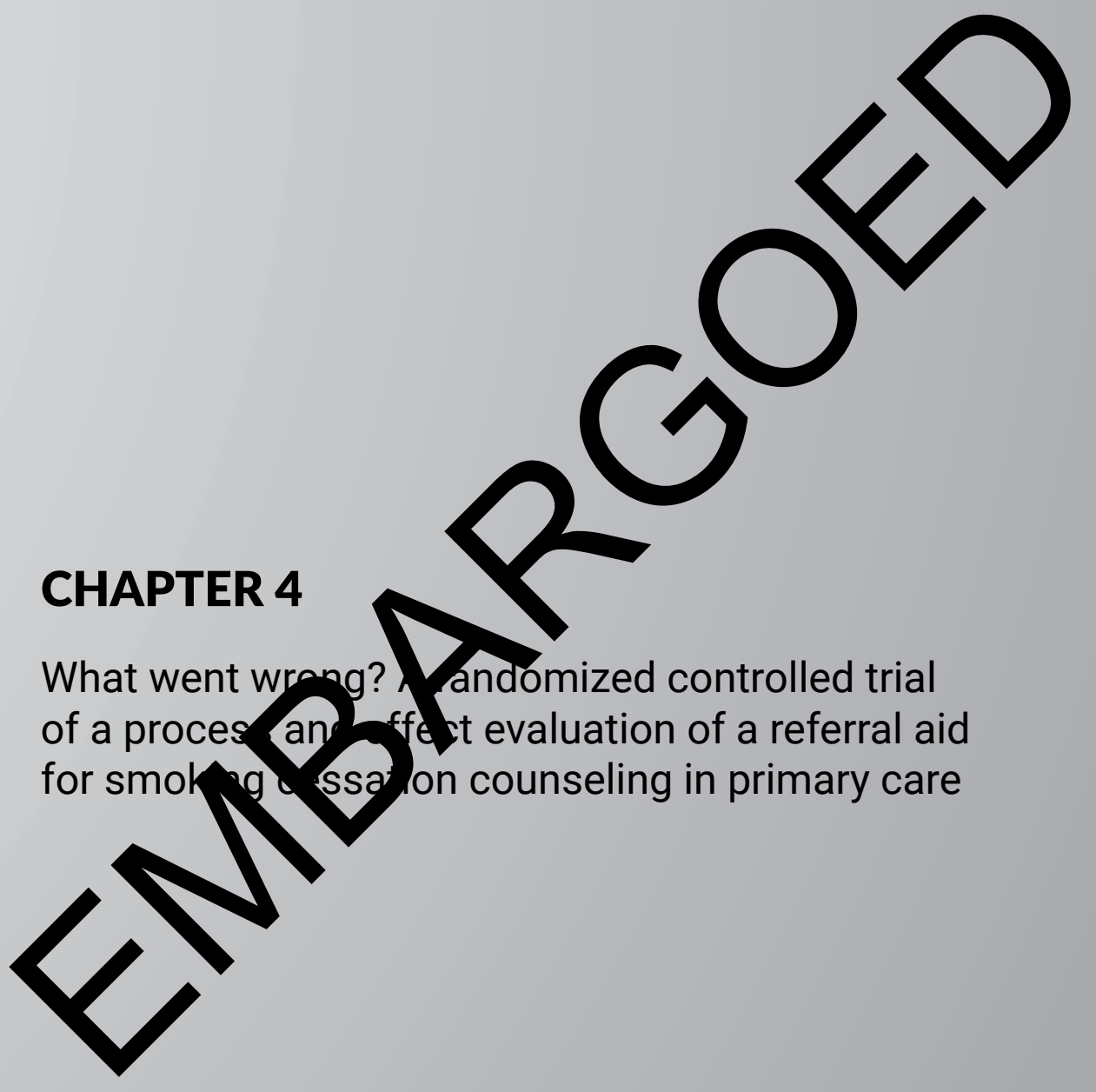

This chapter has been submitted for publication as:

Zijlstra, D.N., Muris, J. W. M., Bolman, C. A. W. \& De Vries, H. (2021).

What went wrong? A randomized controlled trial of a process and effect evaluation of a referral aid for smoking cessation counseling in primary care. 


\section{CHAPTER 5}

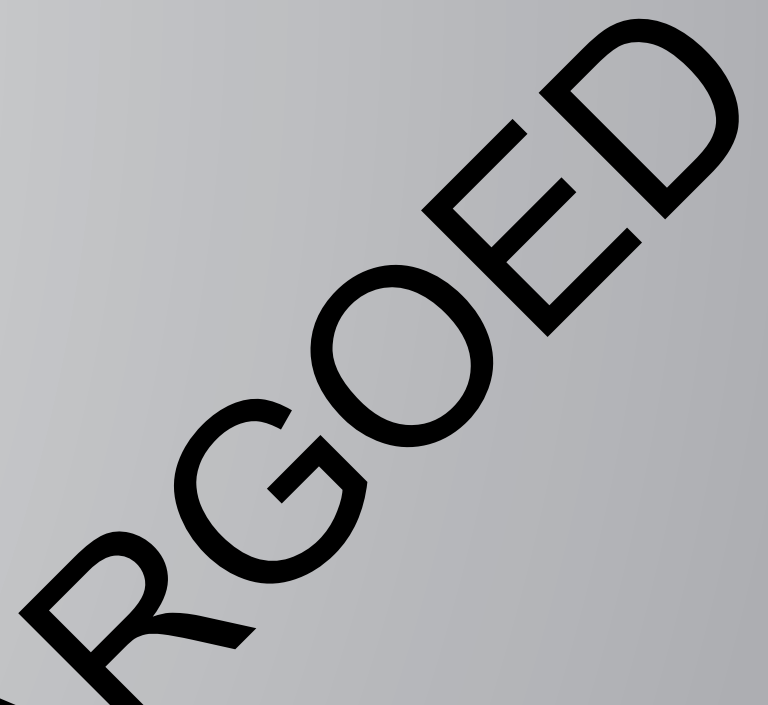

How to convine m primary care professionals to adopt a lue lin Facilitates a ba riers

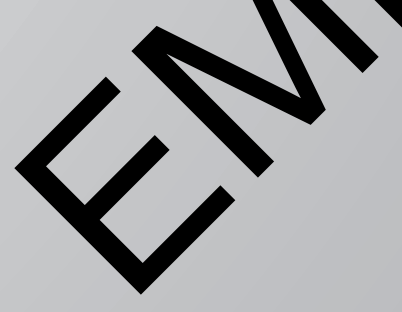

This chapter has been submitted for publication as:

Zijlstra, D. N., Bolman, C. A. W., Muris, J. W. M. \& De Vries, H. (2021).

How to convince more primary care professionals to adopt a valued smoking cessation tool: Facilitators and barriers. 
PART TWO 
Future applications and possibilities 


\section{CHAPTER 6}

Decision aids to facilitate decision making around behavior change in the field of health promotion: A scoping review

This chapter has been published as:

Gültzow, T., Zijlstra, D. N., Bolman, C. A. W., de Vries, H., Dirksen, C. D., Muris, J. W. M. Muris, Smit, E. S. \& Hoving, C. (2021). Decision aids to facilitate decision making around behavior change in the field of health promotion: A scoping review. Patient Education and Counseling. 


\section{ABSTRACT}

Objective: To broadly synthesize literature regarding decision aids (DAs) supporting decision making about diet, physical activity, sleeping, and substance use a scoping review was performed.

Methods: Multiple sources were used: (1 Scientific literature searches, (2 excluded references from a Cochrane review regarding DAs for treatments and screenings, and (3 results from additional searches. Interventions had to ( 1 support informed decision making and ( 2 provide information and help to choose between at least two options. Two researchers screened titles and abstracts. Relevant information was extracted descriptively.

Results: Thirty-five scientific articles and four DAs (grey literature) were included. Results were heterogeneous. Twenty-nine (94\%) studies described substance use DAs. All DAs offered information and value and/or preference clarification. Many other elements were included (e.g., goal-setting). DA's effects were mixed. Few studies used standardized measures, e.g., decisional conflict $(n=4,13 \%)$. Some positive behavioral effects were reported: e.g., smoking abstinence $(n=1)$.

Conclusions: This research shows only some positive behavioral effects of DAs. However, studies reported heterogeneous results/outcomes, impeding knowledge synthesis. Areas of improvement were identified, e.g., establishing which intervention elements are effective regarding health behavior decision making.

Practice DAs can potentially be beneficial in supporting people to change health Implications: behaviors - especially regarding smoking. 


\section{INTRODUCTION}

Noncommunicable diseases continue to be the leading cause of deaths worldwide, inflicting heavy economic burden (259). These diseases' main modifiable risk factors (i.e., blood pressure, blood glucose, cholesterol, and weight) are heavily influenced by individual health behaviors, e.g., tobacco use, physical activity (PA), diet, alcohol use and sleep (duration) (260-263). The occurrence of noncommunicable diseases can therefore be greatly reduced by changing these preventive health-related behaviors (for the sake of readability, we will use this term when referring to tobacco use, PA, diet, alcohol use, and sleep (duration) together).

In all these areas people face decision-making situations, such as deciding to check how well one is meeting behavioral recommendations or deciding whether or not to engage in actions to change an unhealthy behavior. In addition to these decisions, people are confronted with decisions between different possible actions to change their behavior, e.g., people wishing to stop smoking can choose between several effective cessation aids (66, $71,72,264,265)$.

When multiple options exist and persons need to identify their own values (i.e., how (un)desirable certain options' characteristics are (266)) and preferences (i.e., how (un) desirable certain options themselves are taking values into account (266)), decisions are referred to as "preference-sensitive" (127). This type of decision requires that people weigh the benefits and harms of each option on basis of their own values and preferences, since no option is objectively better than others (127). In practice, it requires lay persons to gather available evidence, evaluate its quality and incorporate this information to assess which options fit their values and preferences best - tasks which can be difficult (128).

People facing such preference-sensitive decisions about preventive health-related behaviors may profit from support in their decision-making process, for instance by using decision aids (DAs). DAs are typically used to inform users about available options and their respective characteristics (e.g., effect, time investment and availability) in a balanced manner and help users to choose options that are value- and preference-concordant (127, 267 ), in other words they help users to make informed decisions (268). DAs structure the decision-making process with the help of value clarification methods (VCMs, previously also referred to as value clarification exercises or VCEs) (129) - which can be implicit (i.e., not including overt activity) or explicit (i.e., including overt activity) (269). Such DAs, when applied to treatment or screening decisions (e.g., decisions about cancer treatment options), have shown to have a positive impact on knowledge, accuracy of risk perception, valuesconcordant choices, decisional conflict, feelings of being undecided, costs and the number of people making a decision (127). However, it is unclear whether this promising approach to decision support can also help individuals make informed decisions about preventive health-related behaviors. 


\section{CHAPTER 6}

The most comprehensive knowledge synthesis in the field of DAs excluded studies conducted around DAs focusing on lifestyle (127). However, a systematic review by Moyo et al. (270) has shown that DAs could be a promising approach to smoking cessation, as have individual studies (e.g., (91)). Currently, there is a lack of concrete knowledge of DAs in the broader area of preventive health-related behaviors. To the best of our knowledge, no knowledge synthesis of any kind has been carried out to fill this knowledge gap. We therefore do not know for which preventive health-related behaviors DAs actually exist. In the recent past, studies have been carried out to examine intervention elements (271) of DAs in general and the theoretical basis (272) of treatment and screening DAs in more detail. Effects of DAs focused on treatment and screening decisions are also routinely synthesized in the aforementioned comprehensive knowledge synthesis in the form of a Cochrane review (127) and at least one systematic review has investigated DAs costeffectiveness in general (273). However, all of this information is not available regarding DAs aimed at making decisions about changing preventive health-related behaviors specifically.

Consequently, our aim was to broadly synthesize existing literature in the form of a scoping review by reviewing information regarding DAs supporting informed decision making about these behaviors, focusing on their characteristics, intervention elements, theoretical foundations and (cost-)effectiveness. The synthesized knowledge will be of value to guide future research directions, but also to inform (clinical) practice and to better understand the usefulness of DAs that focus on preventive health-related behavior change.

\section{METHODS}

The methodological framework developed by Arksey \& O'Malley (274), the Joanna Briggs Institute Reviewers' Manual (275) and the Preferred Reporting Items for Systematic Reviews and Meta-Analyses Extension for Scoping Reviews (PRISMA-ScR) (276) guided the study protocol - which can be found on the Open Science Framework (https://osf.io/9xkbv/) (277). However, one change was made: We decided to gather descriptive data instead of quantitative data as the heterogeneity of the results hindered us to conduct quantitative analyses. This made it impossible to calculate Cohen's kappa (however, other measures were taken to ensure reliability, see 2.2 Study and DA selection). Consequently, the data are therefore presented descriptively in text and/or tabular form. The completed PRISMA-ScR checklist can be found in Appendix A.

\subsection{Information sources}

Multiple sources were used to gather data: (1 Scientific literature search results, (2 the excluded publications from the Cochrane review on DAs for people facing health treatment 
or screening decisions (127) (mentioned in the introduction) and (3 results from additional searches, such as a literature search on Google Scholar and a grey literature search on the Ottawa Hospital Research Institute Patient Decision Aid inventory (DALI) (278).

\subsubsection{Scientific literature searches}

Systematic literature searches were conducted in three relevant databases (i.e., PubMed, PsycINFO, and CINAHL) with search strings related to the aforementioned behaviors combined with "decision aid" (for the full overview see tables B.1 and B.2 in Appendix B) in October 2018. Terms were included to exclude papers that focus on policy decision making as the focus of this scoping review was on individual decision making. Searches were restricted to publications pertaining to humans (again, due to the focus on individual human decision making) between January 2008 and October 2018 (to synthesize the most recent literature). Search strategies were specified to each database and discussed with a scientific information specialist.

\subsubsection{Excluded publications from the Cochrane review}

As noted in the introduction, the most comprehensive knowledge synthesis in the field of DAs (the systematic review by Stacey et al. (127)) excluded articles describing DAs focusing on lifestyle - hence, those which were of interest for this scoping review. Therefore, all of those excluded publications were retrieved.

\subsubsection{Additional searches}

Using Google Scholar, we applied a systematic search (see table B.3 in Appendix B for the search strings). Publications within the first 50 hits were screened for each search string. Again, this search was limited to the last 10 years (January 2008 and October 2018). We also created a Google Scholar Alert to inform us of any other relevant publications. Subsequently, we searched through the DALI (278) using all the search terms described above.

Finally, we tried to identify any DAs (in development) that were missed. For this purpose additional strategies were: (1 Cross-referencing included articles and articles only selected for full text screening (see 2.2 Study and DA selection, e.g., (270)), (2 checking the publications from first authors of included articles, (3 using Google Scholar's "related articles"-function and (4 using our existing professional network (e.g., by making use of newsletters of professional associations) and contacting authors of known DAs in development or with currently unpublished findings.

\subsection{Article and DA selection}

Retrieved titles and abstracts were screened by TG and DZ by using the following inclusion criteria: Articles had to describe interventions that (1 supported informed decision making 


\section{CHAPTER 6}

in relation to preventive health-related behaviors and ( 2 provided information about the decision at hand and helped to choose between at least two options (e.g., by including VCMs) $(279,280)$. Articles describing (clinical) treatment DAs were excluded. Inconsistencies between the two reviewers were resolved by discussion. If an agreement could not be reached, $\mathrm{CH}$ helped to come to a conclusion. The selected full articles were assessed by DZ and TG, after which TG extracted all relevant information descriptively which was charted within an Excel spreadsheet developed a priori. After completion of the data extraction by TG, DZ reviewed $10 \%$ of the articles to ensure reliability. Inconsistencies were discussed between TG and DZ. The same procedure was applied to the DAs not found in scientific literature (i.e., grey literature), except for a change in author responsibilities, i.e., DZ initially abstracting the data and TG reviewing $10 \%$. The charting of the information was based on the Cochrane review on treatment and screening DAs (see Appendix C) (127). Authors of the included articles were not contacted to clarify or add information.

\section{RESULTS}

\subsection{Scientific literature}

\subsubsection{Descriptives and study characteristics}

Through this scoping review 35 articles (281-315) were identified, including four study protocols $(289,296,299,302)$. It was not possible to determine the exact number of DAs described in the 35 articles due to a lack of clear identification of DAs by name or other distinguishing characteristic in the majority of the articles. Therefore, the units of analysis for this scoping review were individual studies (not DAs) with the exception of protocol papers which were analyzed together with their associated effect papers. More than half of the studies were of American origin ( $n=16,52 \%)(282,289,294-298,304-309,312-315)$. The main focus was on substance use $(n=29,94 \%)(283-315)$ with 11 DAs solely focusing on smoking $(35 \%)(302-309,311-313,315)$. All studies described DAs that included both information provision and value clarification or described such DA content without explicitly using the terms. All developed DAs contained a multitude of other intervention elements, such as personal stories (306) or encouragement to set a quit date $(302,303)$. For an overview of the included articles see table 1 , for an overview of intervention elements see table 2, and for a flow diagram depicting the selection process see figure 1 . 


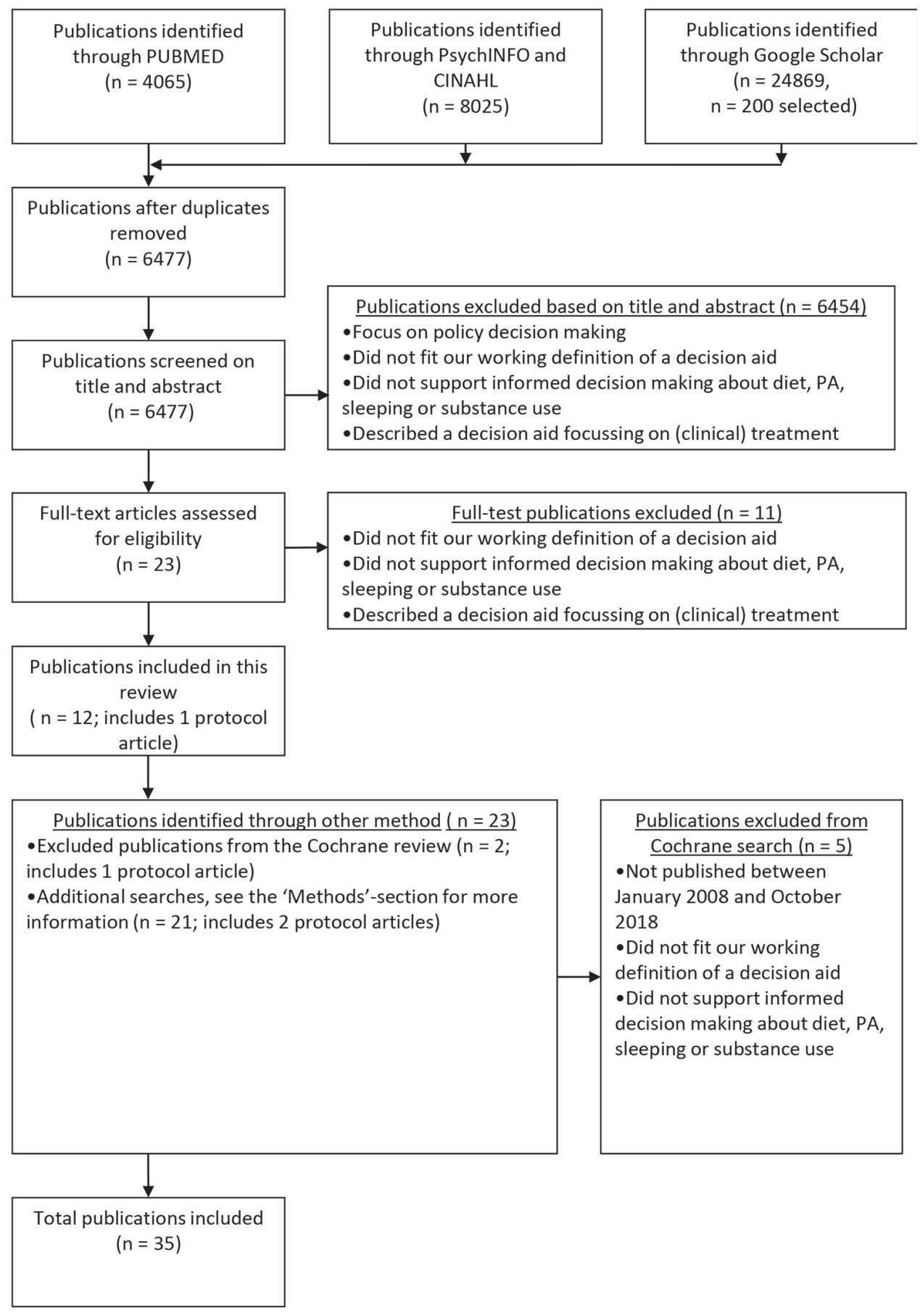

Figure 1. Flow diagram. 
CHAPTER 6

Table 1. Article characteristics

\begin{tabular}{|c|c|c|c|c|}
\hline Article & $\begin{array}{l}\text { Study design/ } \\
\text { methodology }\end{array}$ & Study population & Study aims and purposes & Country of origin \\
\hline $\begin{array}{l}\text { Cupples et al. } \\
\text { (2018) (281) }\end{array}$ & $\begin{array}{l}\text { Mixed method } \\
\text { feasibility study }\end{array}$ & $\begin{array}{l}\text { Patients aged } \geq 18 \text { years with (or at } \\
\text { risk of) coronary heart disease }(\mathrm{CHD})\end{array}$ & $\begin{array}{l}\text { To test the feasibility of using a novel, } \\
\text { paper-based decision tool, to } \\
\text { facilitate shared decision making } \\
\text { (SDM, between HP and patient) in the } \\
\text { process of initiating behavior change } \\
\text { for cardiovascular disease (CVD) } \\
\text { prevention among patients with, or at } \\
\text { high risk of, CHD in general practice }\end{array}$ & $\begin{array}{l}\text { United Kingdom } \\
\text { (UK) }\end{array}$ \\
\hline $\begin{array}{l}\text { Geller et al. (2012) } \\
(282)\end{array}$ & $\begin{array}{l}\text { Not explicitly } \\
\text { mentioned, probably } \\
\text { pre-post pilot study }\end{array}$ & $\begin{array}{l}\text { Older ethnically diverse population } \\
\text { adults visiting two community } \\
\text { housing sites in Hawaii }\end{array}$ & $\begin{array}{l}\text { To test the implementation of a } \\
\text { decisional balance sheet PA program } \\
\text { and fruit and vegetable program, } \\
\text { specifically describing the efficiency } \\
\text { and effectiveness of the programs } \\
\text { adapted for older adults residing in } \\
\text { community living homes }\end{array}$ & $\begin{array}{l}\text { United States of } \\
\text { America (USA) }\end{array}$ \\
\hline $\begin{array}{l}\text { Hirsch et al. (2010) } \\
(283)\end{array}$ & $\begin{array}{l}\text { Pragmatic cluster } \\
\text { randomized } \\
\text { controlled trial } \\
\text { (CRT) }\end{array}$ & $\begin{array}{l}\text { Patients who had their cholesterol } \\
\text { levels measured during a period of } 4 \\
\text { weeks }\end{array}$ & $\begin{array}{l}\text { To evaluate the satisfaction level of } \\
\text { both patients and physicians in a } \\
\text { reciprocal relationship of SDM using } \\
\text { a structured tool for cardiovascular } \\
\text { prevention contrasted to the results } \\
\text { of a control group }\end{array}$ & Germany \\
\hline $\begin{array}{l}\text { Hirsch et al. (2011) } \\
(285)\end{array}$ & $\begin{array}{l}\text { Mixed method } \\
\text { evaluation study }\end{array}$ & $\begin{array}{l}\text { German patients that visited their GP } \\
\text { and had to make a decision which } \\
\text { was covered by the decision aid (DA) }\end{array}$ & $\begin{array}{l}\text { To evaluate the acceptance of SDM } \\
\text { with reference to an interactive, } \\
\text { transactional, and evidence-based } \\
\text { library of DAs by patients and } \\
\text { physicians in the primary care context }\end{array}$ & Germany \\
\hline $\begin{array}{l}\text { Hirsch et al. (2011) } \\
(284)\end{array}$ & Pragmatic CRT & $\begin{array}{l}\text { Patients who had their cholesterol } \\
\text { levels measured during a period of } 4 \\
\text { weeks }\end{array}$ & $\begin{array}{l}\text { To evaluate methodological } \\
\text { difficulties in calculating the } \\
\text { correspondence between patient and } \\
\text { physician satisfaction ratings and to } \\
\text { show the relevance for SDM research }\end{array}$ & Germany \\
\hline $\begin{array}{l}\text { Hirsch et al. (2012) } \\
(287)\end{array}$ & $\begin{array}{l}\text { Mixed method } \\
\text { evaluation study }\end{array}$ & $\begin{array}{l}\text { German patients that visited their GP } \\
\text { and had to make a decision which } \\
\text { was covered by the DA }\end{array}$ & $\begin{array}{l}\text { To evaluate associations between the } \\
\text { use of an interactive, transactional, } \\
\text { and evidence-based library of DAs } \\
\text { and communication and decision } \\
\text { making in patients and physicians in } \\
\text { the primary care context }\end{array}$ & Germany \\
\hline $\begin{array}{l}\text { Hirsch et al. (2012) } \\
(286)\end{array}$ & $\begin{array}{l}\text { Mixed method } \\
\text { evaluation study }\end{array}$ & $\begin{array}{l}\text { German patients that visited their GP } \\
\text { and had to make a decision which } \\
\text { was covered by the DA }\end{array}$ & $\begin{array}{l}\text { To evaluate the uptake of an } \\
\text { interactive, transactional, and } \\
\text { evidence-based library of DAs and its } \\
\text { association to decision making in } \\
\text { patients and physicians in the primary } \\
\text { care context }\end{array}$ & Germany \\
\hline $\begin{array}{l}\text { Hirsch et al. (2012) } \\
(288)\end{array}$ & $\begin{array}{l}\text { Not explicitly } \\
\text { mentioned, analyses } \\
\text { of log data }\end{array}$ & $\begin{array}{l}\text { German patients that visited their GP } \\
\text { and had to make a decision which } \\
\text { was covered by the DA }\end{array}$ & $\begin{array}{l}\text { To examine user interactions of } \\
\text { primary-care physicians and their } \\
\text { patients with the electronic library of } \\
\text { DAs used during consultations, on the } \\
\text { basis of log data }\end{array}$ & Germany \\
\hline $\begin{array}{l}\text { Koelewijn-van Loon } \\
\text { et al. (2008) } \\
\text { (Protocol paper) \& } \\
\text { Koelewijn-van Loon } \\
\text { et al. }(2009) \text { (Effect } \\
\text { paper) }(289,290)\end{array}$ & CRT & $\begin{array}{l}\text { Adult patients eligible for } \\
\text { cardiovascular risk management who } \\
\text { met one or more of the following } \\
\text { criteria blood pressure } \geq 140 \mathrm{~mm} \mathrm{Hg} \\
\text { or receiving treatment for high blood } \\
\text { pressure; total cholesterol } \geq 6.5 \\
\text { mmol/L or receiving treatment for } \\
\text { high cholesterol; smoker aged } \geq 50 \\
\text { years (men) or } \geq 55 \text { years (women); } \\
\text { diabetes; a positive family history of } \\
\text { cardiovascular disease; and visible } \\
\text { obesity (based on the physician's } \\
\text { opinion) }\end{array}$ & $\begin{array}{l}\text { To investigated whether a nurse-led } \\
\text { intervention in primary care had a } \\
\text { positive effect on lifestyle and } 10 \text {-year } \\
\text { cardiovascular risk }\end{array}$ & $\begin{array}{l}\text { Netherlands and the } \\
\text { UK }\end{array}$ \\
\hline
\end{tabular}




\begin{tabular}{llll} 
Behavior (general) & Behavior (specific) & DA Delivery & $\begin{array}{l}\text { Duration to complete Sources of funding } \\
\text { the DA }\end{array}$ \\
\hline $\begin{array}{l}\text { Dietary behavior \& physical } \\
\text { activity (PA) }\end{array}$ & Not described & $\begin{array}{l}\text { Paper-based, used during } \\
\text { consultation with their general } \\
\text { practitioner (GP) }\end{array}$ & $\begin{array}{l}\text { Approximately 15 } \\
\text { minutes (whole } \\
\text { consultation) }\end{array}$
\end{tabular}

$\begin{array}{ll}\text { Dietary behavior \& PA } & \begin{array}{l}\text { Increasing PA and/or (daily) } \\ \text { fruit and vegetable } \\ \text { consumption }\end{array}\end{array}$

$\begin{array}{lll}\begin{array}{l}\text { Combination of paper-based } \\ \text { materials and group } \\ \text { discussions, delivered in }\end{array} & 30-40 \text { minutes } & \begin{array}{l}\text { The National Cancer } \\ \text { Institute (USA) }\end{array}\end{array}$

$\begin{array}{ll}\text { fruit and vegetable } & \text { materials and group } \\ \text { consumption } & \text { discussions, delivered in }\end{array}$

community housing sites,

used in groups (see other

included elements for more

information)

Included multiple

cardiovascular prevention

strategies,

three of which were preventive smoking (cessation)

(dietary behavior, PA \&

substance use)

Modular library that contained

multiple DAs: The DA for

cardiovascular prevention was

the only one that focused on

preventive health-related

behaviors, it included dietary

behavior, PA \& substance use

Included multiple

cardiovascular prevention

strategies,

three of which were preventive

health-related behaviors

(dietary behavior, PA \&

substance use)

Modular library that contained

multiple DAs: The DA for

cardiovascular prevention was

the only one that focused on

preventive health-related

behaviors, it included dietary

behavior, PA \& substance use

Modular library that contained multiple DAs: The DA for

cardiovascular prevention is

the only one that focused on

preventive health-related

behaviors, it included dietary

behavior, PA \& substance use

Modular library that contained multiple DAs: The DA for cardiovascular prevention was

the only one that focused on

preventive health-related

behaviors, it included dietary

behavior, PA \& substance use

Substance use, dietary

behavior \& PA
Ambiguous, but in all

likelihood the same as in

Krones et al. (292): Eating fish

$2 x$ per week (or Omega-3 fatty

acids), exercise $2-3 x$ per week

$>30$ minutes, smoking

(cessation)

Ambiguous, but in all

likelihood the same as in

Krones et al.(292): Eating fish

$2 x$ per week (or Omega- 3 fatty

acids), exercise 2-3x per week

$>30$ minutes, smoking

(cessation)

Ambiguous, but in all

likelihood the same as in

Krones et al. (292): Eating fish

$2 x$ per week (or Omega- 3 fatty

acids), exercise 2-3x per week

$>30$ minutes, smoking

(cessation)

Ambiguous, but in all

likelihood the same as in

Krones et al. (292): Eating fish

$2 x$ per week (or Omega-3 fatty

acids), exercise 2-3x per week

$>30$ minutes), smoking

(cessation)

Ambiguous, but in all

likelihood the same as in

Krones et al. (292): Eating fish

$2 x$ per week (or Omega- 3 fatty

acids), exercise $2-3 x$ per week

$>30$ minutes, smoking

(cessation)

Smoking, alcohol use,

saturated fat intake, fruit, and

vegetable consumptions \& PA
Paper-based, used during

consultation at the GPs

Not reported

Federal Ministry of

Education and

Research (Germany)

$\begin{array}{lll}\text { Digital-based, used during } & \text { Not reported } & \text { Federal Ministry of } \\ \text { consultation at the GP } & & \text { Education and } \\ & \text { Research (Germany) }\end{array}$

$\begin{array}{lll}\text { Ambiguous, but in all } & \text { Not reported } & \text { Federal Ministry of } \\ \text { likelihood paper-based, used } & & \text { Education and } \\ \text { during consultation at the GPs } & & \text { Research (Germany) }\end{array}$

$\begin{array}{lll}\text { Digital-based, used during } & \text { Not reported } & \text { Federal Ministry of } \\ \text { consultation at the GPs } & & \text { Education and } \\ & \text { Research (Germany) }\end{array}$

$\begin{array}{lll}\begin{array}{l}\text { Digital-based, used during } \\ \text { consultation at the GPs }\end{array} & \text { Approximately } 8 & \text { Federal Ministry of } \\ \text { minutes on average } & \text { Education and } \\ \text { Research (Germany) }\end{array}$

$\begin{array}{lll}\begin{array}{l}\text { Digital-based, used during } \\ \text { consultation at the GPs }\end{array} & \text { Approximately } 8 & \text { Federal Ministry of } \\ & \text { minutes on average } & \text { Education and } \\ & & \text { Research (Germany) }\end{array}$

Paper-based, delivered during Not reported (for the Netherlands a primary care consultation, DA alone) Organisation for had to be read at home Health Research and (between two consultations)
Development (ZonMw,

Netherlands) and

Maastricht University

(Netherlands) 
CHAPTER 6

Table 1. Continued

\begin{tabular}{|c|c|c|c|c|}
\hline Article & $\begin{array}{l}\text { Study design/ } \\
\text { methodology }\end{array}$ & Study population & Study aims and purposes & Country of origin \\
\hline $\begin{array}{l}\text { Koelewijn-van Loon } \\
\text { et al. (2010) (291) }\end{array}$ & CRT & $\begin{array}{l}\text { Adult patients eligible for } \\
\text { cardiovascular risk management who } \\
\text { met one or more of the following } \\
\text { criteria blood pressure } \geq 140 \mathrm{~mm} \mathrm{Hg} \\
\text { or receiving treatment for high blood } \\
\text { pressure; total cholesterol } \geq 6.5 \\
\mathrm{mmol} / \mathrm{L} \text { or receiving treatment for } \\
\text { high cholesterol; smoker aged } \geq 50 \\
\text { years (men) or } \geq 55 \text { years (women); } \\
\text { diabetes; a positive family history of } \\
\text { cardiovascular disease; and visible } \\
\text { obesity (based on the physician's } \\
\text { opinion) }\end{array}$ & $\begin{array}{l}\text { To investigate the short-term effect of } \\
\text { their nurse-led intervention on } \\
\text { patients' risk perception and lifestyle, } \\
\text { in comparison with usual nurse-led } \\
\text { care }\end{array}$ & $\begin{array}{l}\text { Netherlands and the } \\
\text { UK }\end{array}$ \\
\hline $\begin{array}{l}\text { Krones et al. (2008) } \\
(292)\end{array}$ & Pragmatic CRT & $\begin{array}{l}\text { Patients who had their cholesterol } \\
\text { levels measured during a period of } 4 \\
\text { weeks }\end{array}$ & $\begin{array}{l}\text { To evaluate the effectiveness of the } \\
\text { DA as judged by patients }\end{array}$ & $\begin{array}{l}\text { Germany and } \\
\text { Austria }\end{array}$ \\
\hline $\begin{array}{l}\text { Krones et al. (2010) } \\
(293)\end{array}$ & Pragmatic CRT & $\begin{array}{l}\text { Patients in whom discussion of } \\
\text { preventive measures seemed } \\
\text { indicated }\end{array}$ & $\begin{array}{l}\text { To assess the feasibility and outcome } \\
\text { of measuring the theory of planned } \\
\text { behavior (TPB) in patients receiving } \\
\text { routine counselling versus } \\
\text { counselling with a DA during primary } \\
\text { care consultation on cardiovascular } \\
\text { risk prevention }\end{array}$ & $\begin{array}{l}\text { Germany and } \\
\text { Austria }\end{array}$ \\
\hline $\begin{array}{l}\text { Sheridan et al. } \\
\text { (2013) (Protocol } \\
\text { paper) \& Keyserling } \\
\text { et al. }(2014) \text { (Effect } \\
\text { paper) }(296,298)\end{array}$ & $\begin{array}{l}\text { Comparative } \\
\text { effectiveness trial }\end{array}$ & $\begin{array}{l}\text { Patients at participating practices } \\
\text { (seen for an office visit within the } \\
\text { past } 2 \text { years), age } 35-79 \text {, and at high } \\
\text { risk for } \mathrm{CHD} \text { (angina, MI, or } \mathrm{CHD} \\
\text { death) defined by a Framingham risk } \\
\text { score of } \geq 10 \% \text { or known CVD }\end{array}$ & $\begin{array}{l}\text { To assess the effectiveness, } \\
\text { acceptability, and cost-effectiveness } \\
\text { of a combined lifestyle and } \\
\text { medication intervention to reduce } \\
\text { CHD risk offered in counselor- } \\
\text { delivered and web-based formats }\end{array}$ & USA and Singapore \\
\hline $\begin{array}{l}\text { Tinsel et al. (2017) } \\
\text { (Protocol paper) \& } \\
\text { Tinsel et al. (2018) } \\
\text { (effect paper) (299, } \\
300)\end{array}$ & $\begin{array}{l}\text { Two-arm, } \\
\text { randomized, } \\
\text { controlled pilot } \\
\text { study }\end{array}$ & $\begin{array}{l}\text { Patients with at least one } \\
\text { cardiovascular risk factor } \\
\text { (hypertension, hypercholesteremia, } \\
\text { diabetes, arteriosclerosis, smoking, } \\
\text { obesity, high stress level or drug } \\
\text { prescription against hypertension, } \\
\text { high cholesterol) }\end{array}$ & $\begin{array}{l}\text { To test the intervention regarding its } \\
\text { usability, acceptance, and potential } \\
\text { effects in primary care and to test the } \\
\text { feasibility of the randomized study } \\
\text { design }\end{array}$ & Germany \\
\hline $\begin{array}{l}\text { Van Steenkiste et al. } \\
(2008)(301)\end{array}$ & $\begin{array}{l}\text { Cross-sectional } \\
\text { study }\end{array}$ & $\begin{array}{l}\text { Patients (aged } 40-75 \text { years) without } \\
\text { established CVD who were at high, or } \\
\text { at potentially high-cardiovascular risk }\end{array}$ & $\begin{array}{l}\text { To assess patients' responsiveness } \\
\text { to a decision support tool for primary } \\
\text { prevention of CVDs }\end{array}$ & Netherlands \\
\hline $\begin{array}{l}\text { BinDhim et al. } \\
\text { (2014) (Protocol } \\
\text { paper) \& BinDhim } \\
\text { etl al. }(2018) \text { (Effect } \\
\text { paper) }(302,303)\end{array}$ & $\begin{array}{l}\text { Automated, } \\
\text { double-blind } \\
\text { randomized } \\
\text { controlled trial } \\
(\mathrm{RCT})\end{array}$ & $\begin{array}{l}\text { Self-selected adult ( } \geq 18 \text { years old) } \\
\text { daily smokers from the USA, } \\
\text { Australia, Singapore, and the UK }\end{array}$ & $\begin{array}{l}\text { To test the efficacy of an interactive } \\
\text { smoking cessation DA app compared } \\
\text { with a smoking cessation static } \\
\text { information app on quit rates }\end{array}$ & $\begin{array}{l}\text { Saudi Arabia and } \\
\text { Australia }\end{array}$ \\
\hline $\begin{array}{l}\text { Brunette et al. } \\
(2011)(304)\end{array}$ & Quasi experiment & $\begin{array}{l}\text { Adult smokers with severe mental } \\
\text { illnesses who were receiving } \\
\text { supported housing and } \\
\text { comprehensive psychiatric services } \\
\text { at two settings within a large, urban, } \\
\text { psychosocial rehabilitation center }\end{array}$ & $\begin{array}{l}\text { To test the effectiveness of the first } \\
\text { version of their motivational tool }\end{array}$ & USA \\
\hline
\end{tabular}




$\begin{array}{lllll}\text { Behavior (general) } & \text { Behavior (specific) } & \text { DA Delivery } & \begin{array}{l}\text { Duration to complete } \\ \text { the DA }\end{array} & \begin{array}{l}\text { Sources of funding } \\ \text { Substance use, dietary }\end{array} \\ \begin{array}{llll}\text { Sehoking, alcohol use, } \\ \text { saturated fat intake, fruit, and } \\ \text { vegetable consumptions \& PA }\end{array} & \begin{array}{l}\text { Paper-based, delivered during } \\ \text { a primary care consultation, } \\ \text { had to be read at home } \\ \text { (between two consultations) }\end{array} & \begin{array}{l}\text { Not reported } \\ \end{array} & & \begin{array}{l}\text { Netherlands } \\ \text { Organisation for } \\ \text { Health Research and } \\ \text { Development (ZonMw } \\ \end{array} \\ & & \text { Netherlands) and } \\ & & \text { Maastricht University } \\ & & \text { (Netherlands) }\end{array}$

\begin{tabular}{|c|c|c|c|c|}
\hline $\begin{array}{l}\text { Included multiple } \\
\text { cardiovascular prevention } \\
\text { strategies, three of which were } \\
\text { preventive health-related } \\
\text { behaviors (dietary behavior, PA } \\
\text { \& substance use) }\end{array}$ & $\begin{array}{l}\text { Eating fish } 2 x \text { per week (or } \\
\text { Omega- } 3 \text { fatty acids), exercise } \\
2-3 x \text { per week }>30 \text { minutes, } \\
\text { smoking (cessation) }\end{array}$ & $\begin{array}{l}\text { Ambiguous, but in all } \\
\text { likelihood paper-based, used } \\
\text { during consultation at the GPs }\end{array}$ & Not reported & $\begin{array}{l}\text { Federal Ministry of } \\
\text { Education and } \\
\text { Research (Germany) }\end{array}$ \\
\hline $\begin{array}{l}\text { Included multiple } \\
\text { cardiovascular prevention } \\
\text { strategies, } \\
\text { three of which were preventive } \\
\text { health-related behaviors } \\
\text { (dietary behavior, PA \& } \\
\text { substance use) }\end{array}$ & $\begin{array}{l}\text { Ambiguous, but in all } \\
\text { likelihood the same as in } \\
\text { Krones et al.(292): Eating fish } \\
2 x \text { per week (or Omega- } 3 \text { fatty } \\
\text { acids), exercise } 2-3 x \text { per week } \\
>30 \text { minutes, smoking } \\
\text { (cessation) }\end{array}$ & $\begin{array}{l}\text { Ambiguous, but in all } \\
\text { likelihood paper-based, used } \\
\text { during consultation at the GPs }\end{array}$ & Not reported & $\begin{array}{l}\text { Ambiguous, but in all } \\
\text { likelihood the same as } \\
\text { in Krones et al. (292): } \\
\text { Federal Ministry of } \\
\text { Education and } \\
\text { Research (Germany) }\end{array}$ \\
\hline $\begin{array}{l}\text { Included multiple } \\
\text { cardiovascular prevention } \\
\text { strategies, three of which were } \\
\text { preventive health-related } \\
\text { behaviors (dietary behavior, PA } \\
\text { \& substance use) }\end{array}$ & $\begin{array}{l}\text { Changing diet (e.g., eating } \\
\text { polyunsaturated fats rather } \\
\text { than reducing total fat } \\
\text { content), increasing PA, } \\
\text { smoking (cessation) }\end{array}$ & $\begin{array}{l}\text { Digital-based, used with the } \\
\text { assistance of a health } \\
\text { counselor }\end{array}$ & Not reported & $\begin{array}{l}\text { U.S. Centers for } \\
\text { Disease Control and } \\
\text { Prevention (USA) and } \\
\text { National Institutes of } \\
\text { Health (USA) }\end{array}$ \\
\hline $\begin{array}{l}\text { Included multiple } \\
\text { cardiovascular prevention } \\
\text { strategies, three of which were }\end{array}$ & $\begin{array}{l}\text { Ambiguous, but in all } \\
\text { likelihood smoking, PA, alcohol } \\
\text { use, changing diet and }\end{array}$ & $\begin{array}{l}\text { Paper-based, received at the } \\
\text { GP }\end{array}$ & Not reported & $\begin{array}{l}\text { German Heart } \\
\text { Foundation (Germany) }\end{array}$ \\
\hline
\end{tabular}

preventive health-related changing sleeping behavior

behaviors (PA, dietary

behavior, substance use, sleep-related behaviors)

Included multiple

cardiovascular prevention
strategies, probably three of

which were preventive

Paper-based, was presented 22 minutes (SD 12 Ambiguous, but in all

likelihood smoking, PA, alcohol during a consultation at the minutes) likelihood The

use and changing diet GP, participants were asked to

Netherlands

health-related behaviors

complete it at home

(dietary behavior, PA \&

substance use)

Organization for

Health Research and

Development (ZonMw, Netherlands) (316)

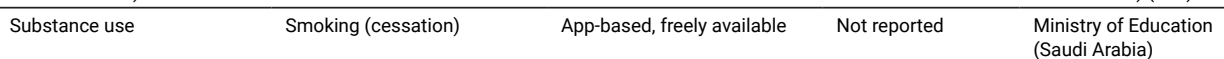


CHAPTER 6

Table 1. Continued

\begin{tabular}{llll}
\hline Article & $\begin{array}{l}\text { Study design/ } \\
\text { methodology }\end{array}$ & Study population & Study aims and purposes \\
\hline $\begin{array}{l}\text { Brunette et al. } \\
(2013)(305)\end{array}$ & RCT & $\begin{array}{l}\text { Daily smokers with a mood or } \\
\text { psychotic disorder with persisting } \\
\text { functional disability, but without other } \\
\text { current substance dependence }\end{array}$ & $\begin{array}{l}\text { To assess whether a single session } \\
\text { of a computerized motivational } \\
\text { decision support system with carbon } \\
\text { monoxide and health checklist } \\
\text { feedback would lead to higher rates } \\
\text { of initiating smoking cessation }\end{array}$ \\
& & $\begin{array}{l}\text { treatment than a version of the } \\
\text { system with health checklist } \\
\text { feedback alone (no carbon monoxide } \\
\text { feedback) }\end{array}$
\end{tabular}

\begin{tabular}{|c|c|c|c|c|}
\hline $\begin{array}{l}\text { Brunette et al. } \\
(2015)(306)\end{array}$ & $\begin{array}{l}\text { Pre-post pilot study, } \\
\text { with a randomly } \\
\text { selected control } \\
\text { group (for which not } \\
\text { all measures were } \\
\text { assessed) }\end{array}$ & $\begin{array}{l}\text { Safety net clinic patients between } \\
18-70 \text { years who smoked four } \\
\text { cigarettes or more per day }\end{array}$ & $\begin{array}{l}\text { To assess whether this web-based, } \\
\text { motivational, decision-support } \\
\text { system could engage smokers who } \\
\text { were not motivated to use treatment } \\
\text { in a primary care 'safety net' clinic } \\
\text { that serves disadvantaged people }\end{array}$ & USA \\
\hline $\begin{array}{l}\text { Cupertino et al. } \\
(2010)(307)\end{array}$ & $\begin{array}{l}\text { Pre-test, post-test } \\
\text { assessment with no } \\
\text { control group }\end{array}$ & $\begin{array}{l}\text { Underserved, low-literacy smokers } \\
\text { ( } 46.7 \% \text { Latinos) }\end{array}$ & $\begin{array}{l}\text { To assess the feasibility and } \\
\text { preliminary outcomes of a } \\
\text { computerized DA to improve } \\
\text { knowledge and utilization of smoking } \\
\text { cessation resources among } \\
\text { underserved, low-literacy smokers }\end{array}$ & USA \\
\hline $\begin{array}{l}\text { Ferron et al. (2011) } \\
(308)\end{array}$ & $\begin{array}{l}\text { Mixed method } \\
\text { usability test }\end{array}$ & $\begin{array}{l}\text { Convenience sample of smokers } \\
\text { between the age of } 18 \text { and } 65\end{array}$ & $\begin{array}{l}\text { To test the usability of the } \\
\text { intervention }\end{array}$ & USA \\
\hline $\begin{array}{l}\text { Ferron et al. (2012) } \\
(309)\end{array}$ & $\begin{array}{l}\text { Secondary analysis } \\
\text { of data from an RCT }\end{array}$ & $\begin{array}{l}\text { Adult smokers with serious mental } \\
\text { illness who were receiving care at an } \\
\text { urban psychiatric rehabilitation center }\end{array}$ & $\begin{array}{l}\text { To study whether cognitive } \\
\text { functioning, clinical characteristics } \\
\text { and computer experience predict time } \\
\text { spent using a web-based DA and } \\
\text { whether these variables predict the } \\
\text { main proximal outcome, engagement } \\
\text { in smoking cessation treatment, and } \\
\text { other quit behaviors }\end{array}$ & USA \\
\hline
\end{tabular}

\begin{tabular}{|c|c|c|c|c|}
\hline $\begin{array}{l}\text { Hollen et al. (2013) } \\
\text { (310) }\end{array}$ & Prospective RCT & $\begin{array}{l}\text { Adolescents ( } 14-19 \text { years) survivors } \\
\text { of childhood cancer who had a } \\
\text { history of cancer diagnosed between } \\
\text { birth and } 12 \text { years but had been } \\
\text { disease-free for at least } 5 \text { years (no } \\
\text { treatment during the past } 2 \text { years) }\end{array}$ & $\begin{array}{l}\text { To test a DA for adolescent survivors } \\
\text { of childhood cancer that is aimed at } \\
\text { difficult decisions related to engaging } \\
\text { in substance use risk behaviors }\end{array}$ & USA \\
\hline $\begin{array}{l}\text { Lee et al. (2016) } \\
\text { (311) }\end{array}$ & CRT & $\begin{array}{l}\text { Adult ( } \geq 18 \text { years old) smokers } \\
\text { visiting an outpatient clinic of a } \\
\text { Department of Family Medicine and a } \\
\text { Health Screening Center }\end{array}$ & $\begin{array}{l}\text { To develop a culturally tailored DA for } \\
\text { smoking cessation and to evaluate its } \\
\text { effect on deciding to use smoking } \\
\text { cessation medication }\end{array}$ & Republic of Korea \\
\hline $\begin{array}{l}\text { McDonnell et al. } \\
(2014)(312)\end{array}$ & $\begin{array}{l}\text { Prospective, } \\
\text { one-group repeated } \\
\text { measures design }\end{array}$ & $\begin{array}{l}\text { Smokers (at least } 21 \text { years) } \\
\text { motivated to quit that were scheduled } \\
\text { for surgery for a suspicious thoracic } \\
\text { mass or known cancer, with a } \\
\text { household family member that also } \\
\text { smoked and was also motivated to } \\
\text { quit }\end{array}$ & $\begin{array}{l}\text { To test the feasibility of a } \\
\text { multidisciplinary, multicomponent, } \\
\text { theory-based DA }\end{array}$ & USA \\
\hline
\end{tabular}




\begin{tabular}{|c|c|c|c|c|}
\hline Behavior (general) & Behavior (specific) & DA Delivery & $\begin{array}{l}\text { Duration to complete } \\
\text { the DA }\end{array}$ & Sources of funding \\
\hline Substance use & Smoking (cessation) & $\begin{array}{l}\text { Digital-based, used together } \\
\text { with a research assistant }\end{array}$ & $30-90$ minutes & $\begin{array}{l}\text { U.S. Department of } \\
\text { Education, National } \\
\text { Institute on Disability } \\
\text { and Rehabilitation } \\
\text { Research } \\
\text { (USA); the Substance } \\
\text { Abuse and Mental } \\
\text { Health Services } \\
\text { Administration, Center } \\
\text { for Mental Health } \\
\text { Services and } \\
\text { Consumer Affairs } \\
\text { Program (USA) and } \\
\text { the Bristol-Myers } \\
\text { Squibb Foundation } \\
\text { (USA) }\end{array}$ \\
\hline Substance use & Smoking (cessation) & $\begin{array}{l}\text { Digital and web-based, used } \\
\text { with a research assistant } \\
\text { present }\end{array}$ & $45-90$ minutes & $\begin{array}{l}\text { Dartmouth SYNERGY } \\
\text { (USA) }\end{array}$ \\
\hline Substance use & Smoking (cessation) & $\begin{array}{l}\text { Digital-based, delivered in } \\
\text { safety-net clinics and } \\
\text { community health fairs }\end{array}$ & Not reported & $\begin{array}{l}\text { The Healthcare } \\
\text { Foundation of Greater } \\
\text { Kansas City (USA) }\end{array}$ \\
\hline Substance use & Smoking (cessation) & $\begin{array}{l}\text { Digital and web-based, used } \\
\text { with a researcher present }\end{array}$ & $\begin{array}{l}47 \text { minutes } \\
(S D=24.6) \text { in the third } \\
\text { and final version }\end{array}$ & $\begin{array}{l}\text { The Foundation for } \\
\text { Informed Medical } \\
\text { Decision Making } \\
\text { (USA) }\end{array}$ \\
\hline Substance use & Smoking (cessation) & $\begin{array}{l}\text { Digital-based, delivered in a } \\
\text { clinic office with research staff } \\
\text { present }\end{array}$ & $\begin{array}{l}32.12-190.3 \text { minutes } \\
(M=92.27, S D=32.77)\end{array}$ & $\begin{array}{l}\text { U.S. Department of } \\
\text { Education, National } \\
\text { Institute on Disability } \\
\text { and Rehabilitation } \\
\text { Research } \\
\text { (USA); the Substance } \\
\text { Abuse and Mental } \\
\text { Health Services } \\
\text { Administration, Center } \\
\text { for Mental Health } \\
\text { Services and } \\
\text { Consumer Affairs } \\
\text { Program (USA) and } \\
\text { the Bristol-Myers } \\
\text { Squibb Foundation } \\
\text { (USA) }\end{array}$ \\
\hline Substance use & $\begin{array}{l}\text { Smoking, alcohol } \\
\text { consumption, and illicit drug } \\
\text { use }\end{array}$ & $\begin{array}{l}\text { As the DA consistent of } \\
\text { multiple components, it was } \\
\text { delivered in multiple ways: See } \\
\text { other included elements (table } \\
\text { 2) for more information }\end{array}$ & $\begin{array}{l}\text { Different modules } \\
\text { varied in length, from } \\
10 \text { to } 60 \text { minutes, the } \\
\text { whole intervention } \\
\text { involved } \\
\text { approximately } 7.5 \\
\text { contact hours } \\
\text { (including } \\
\text { measurements) }\end{array}$ & $\begin{array}{l}\text { National Institute of } \\
\text { Nursing Research } \\
\text { (USA) }\end{array}$ \\
\hline Substance use & Smoking (cessation) & $\begin{array}{l}\text { Video-based (presented on a } \\
\text { tablet computer), was watched } \\
\text { before a consultation at a } \\
\text { department of family medicine }\end{array}$ & 7 minutes & Pfizer (USA) \\
\hline Substance use & Smoking (cessation) & $\begin{array}{l}\text { As the DA consistent of } \\
\text { multiple components, it was } \\
\text { delivered in multiple ways: See } \\
\text { other included elements (table } \\
\text { 2) for more information }\end{array}$ & $\begin{array}{l}\text { Different modules } \\
\text { varied in length, } \\
\text { face-to-face visits } \\
\text { lasted about } 45 \\
\text { minutes, while } \\
\text { optional booster } \\
\text { sessions lasted less } \\
\text { than } 15 \text { minutes }\end{array}$ & $\begin{array}{l}\text { The American Cancer } \\
\text { Society (USA) }\end{array}$ \\
\hline
\end{tabular}


CHAPTER 6

Table 1. Continued

\begin{tabular}{|c|c|c|c|c|}
\hline Article & $\begin{array}{l}\text { Study design/ } \\
\text { methodology }\end{array}$ & Study population & Study aims and purposes & Country of origin \\
\hline $\begin{array}{l}\text { McDonnell et al. } \\
(2016)(313)\end{array}$ & $\begin{array}{l}\text { Prospective, } \\
\text { one-group repeated } \\
\text { measures, } \\
\text { mixed-method } \\
\text { feasibility study }\end{array}$ & $\begin{array}{l}\text { Smokers (at least } 21 \text { years) } \\
\text { motivated to quit that were scheduled } \\
\text { for surgery for a suspicious thoracic } \\
\text { mass or known cancer, with a } \\
\text { household family member that also } \\
\text { smoked and was also motivated to } \\
\text { quit }\end{array}$ & $\begin{array}{l}\text { To determine the feasibility and } \\
\text { acceptability of a multidisciplinary, } \\
\text { theory-based DA, for patients } \\
\text { scheduled to undergo thoracic } \\
\text { surgery and for their family members } \\
\text { who smoke }\end{array}$ & USA \\
\hline $\begin{array}{l}\text { Rhee et al. (2008) } \\
(314)\end{array}$ & Prospective RCT & $\begin{array}{l}\text { Rural adolescents ( } 14-20 \text { years old) } \\
\text { with asthma without learning } \\
\text { disabilities }\end{array}$ & $\begin{array}{l}\text { To determine the feasibility of the } \\
\text { decision-making program for } \\
\text { adolescents with asthma and to } \\
\text { conduct preliminary testing of the } \\
\text { following hypothesis: Adolescents } \\
\text { receiving the intervention, framed } \\
\text { within the context of engaging in risk } \\
\text { behaviors and asthma and its } \\
\text { treatment, would report improved } \\
\text { quality decision making, reduced risk } \\
\text { motivation, and reduced risk } \\
\text { behaviors at } 2,4 \text {, and } 6 \text { months } \\
\text { post-intervention compared with the } \\
\text { control group and to examine whether } \\
\text { intervention effects would vary by } \\
\text { gender or race }\end{array}$ & USA \\
\hline $\begin{array}{l}\text { Sheridan et al. } \\
(2010)(294)\end{array}$ & $\mathrm{RCT}$ & $\begin{array}{l}\text { Convenience sample of men and } \\
\text { women from a registry of participants } \\
\text { interested in decision support testing } \\
\text { between ( } \geq 45 \text { years old) who were } \\
\text { likely to be at moderate to high risk of } \\
\text { heart diseases }\end{array}$ & $\begin{array}{l}\text { To determine whether adding an } \\
\text { explicit VCM* to a DA on heart } \\
\text { disease prevention improved } \\
\text { decision-making outcomes, including } \\
\text { decisional conflict, intent for } \\
\text { screening, perceived value } \\
\text { concordance, and self-efficacy }\end{array}$ & USA \\
\hline $\begin{array}{l}\text { Sheridan et al. } \\
\text { (2011) (295) }\end{array}$ & RCT & $\begin{array}{l}\text { Patients between the ages of } 40-79 \\
\text { years presenting for routine care with } \\
\text { no prior history of cardiovascular } \\
\text { disease, diabetes mellitus, or other } \\
\text { serious medical condition; and were } \\
\text { at moderate or high risk of CHD over } \\
10 \text { years }\end{array}$ & $\begin{array}{l}\text { To test the feasibility of delivering the } \\
\text { intervention in clinical practice and } \\
\text { the effect of the intervention on } \\
\text { important efficacy outcomes }\end{array}$ & USA \\
\hline $\begin{array}{l}\text { Sheridan et al. } \\
\text { (2014) (297) }\end{array}$ & RCT & $\begin{array}{l}\text { Patients between the ages of } 40-79 \\
\text { years presenting for routine care with } \\
\text { no prior history of cardiovascular } \\
\text { disease, diabetes mellitus, or other } \\
\text { serious medical condition; and were } \\
\text { at moderate or high risk of CHD over } \\
10 \text { years }\end{array}$ & $\begin{array}{l}\text { To further understand earlier found } \\
\text { effects }\end{array}$ & USA \\
\hline $\begin{array}{l}\text { Warner et al. (2015) } \\
(315)\end{array}$ & $\begin{array}{l}\text { Randomized, } \\
\text { two-group pilot } \\
\text { study }\end{array}$ & $\begin{array}{l}\text { Smoking patients ( } \geq 18 \text { years old) } \\
\text { scheduled for elective surgery }\end{array}$ & $\begin{array}{l}\text { To develop and pilot test a DA to } \\
\text { increase patient involvement in } \\
\text { decisions regarding smoking } \\
\text { behavior of cigarette smokers } \\
\text { scheduled for elective surgery }\end{array}$ & USA \\
\hline
\end{tabular}

Note. Articles are sorted thematically, alphabetically, and chronologically. Ambiguous information was not verified with the original authors. *Called a value clarification exercise (VCE) in their article 


\begin{tabular}{|c|c|c|c|c|}
\hline Behavior (general) & Behavior (specific) & DA Delivery & $\begin{array}{l}\text { Duration to complete } \\
\text { the DA }\end{array}$ & Sources of funding \\
\hline Substance use & Smoking (cessation) & $\begin{array}{l}\text { As the DA consistent of } \\
\text { multiple components, it was } \\
\text { delivered in multiple ways: See } \\
\text { other included elements (table } \\
\text { 2) for more information }\end{array}$ & Not reported & $\begin{array}{l}\text { The American Cancer } \\
\text { Society (USA) and the } \\
\text { Oncology Nursing } \\
\text { Society Foundation } \\
\text { (USA) }\end{array}$ \\
\hline Substance use & $\begin{array}{l}\text { Smoking, alcohol } \\
\text { consumption, and illicit drug } \\
\text { use }\end{array}$ & $\begin{array}{l}\text { As the DA consistent of } \\
\text { multiple components, it was } \\
\text { delivered in multiple ways: See } \\
\text { other included elements (table } \\
\text { 2) for more information }\end{array}$ & $\begin{array}{l}\text { Different modules } \\
\text { varied in length, from } \\
10 \text { to } 90 \text { minutes }\end{array}$ & $\begin{array}{l}\text { National Institute of } \\
\text { Nursing Research } \\
\text { (USA) }\end{array}$ \\
\hline $\begin{array}{l}\text { Included multiple } \\
\text { cardiovascular prevention } \\
\text { strategies, only one of which } \\
\text { was a preventive health-related } \\
\text { behavior (substance use) }\end{array}$ & Smoking (cessation) & $\begin{array}{l}\text { Digital and web-based, } \\
\text { participants got access to } \\
\text { either the DA with or without } \\
\text { an explicit } \mathrm{VCM}^{\star} \text { alongside a } \\
\text { hypothetical scenario }\end{array}$ & $\begin{array}{l}\text { Without explicit VCM } \\
=5 \text { minutes (range } 1 \\
\text { to } 12 \text { minutes), with } \\
\text { explicit VCM = } 11 \\
\text { minutes (range } 4 \text { to } \\
21 \text { minutes) }\end{array}$ & $\begin{array}{l}\text { The American Heart } \\
\text { Association (USA), the } \\
\text { National Heart Lung } \\
\text { and Blood Institute } \\
\text { (USA), and the } \\
\text { National Cancer } \\
\text { Institute (USA) }\end{array}$ \\
\hline $\begin{array}{l}\text { Included multiple } \\
\text { cardiovascular prevention } \\
\text { strategies, only one preventive } \\
\text { health-related behavior } \\
\text { (substance use) }\end{array}$ & Smoking (cessation) & $\begin{array}{l}\text { Digital and web-based, used in } \\
\text { one university internal } \\
\text { medicine practice, before a } \\
\text { consultation }\end{array}$ & $\begin{array}{l}12 \text { minutes (range: } \\
1-45 \text { minutes) }\end{array}$ & $\begin{array}{l}\text { The American Heart } \\
\text { Association (USA), the } \\
\text { National Heart Lung } \\
\text { and Blood Institute } \\
\text { (USA), and the } \\
\text { National Cancer } \\
\text { Institute (USA) }\end{array}$ \\
\hline $\begin{array}{l}\text { Included multiple } \\
\text { cardiovascular prevention } \\
\text { strategies, } \\
\text { only one of which was a } \\
\text { preventive health-related } \\
\text { behavior (substance use) }\end{array}$ & Smoking (cessation) & $\begin{array}{l}\text { Digital and web-based, used in } \\
\text { one university internal } \\
\text { medicine practice, before a } \\
\text { consultation }\end{array}$ & $\begin{array}{l}12 \text { minutes (range: } \\
<1-45 \text { minutes) }\end{array}$ & $\begin{array}{l}\text { The American Heart } \\
\text { Association (USA), the } \\
\text { National Heart Lung } \\
\text { and Blood Institute } \\
\text { (USA), and the } \\
\text { National Cancer } \\
\text { Institute (USA) }\end{array}$ \\
\hline Substance use & Smoking (cessation) & $\begin{array}{l}\text { Paper-based, delivered in an } \\
\text { examination room of a } \\
\text { preoperative evaluation center } \\
\text { by clinicians that regularly } \\
\text { staff the center }\end{array}$ & 5-10 minutes & $\begin{array}{l}\text { The National Cancer } \\
\text { Institute (USA) }\end{array}$ \\
\hline
\end{tabular}


CHAPTER 6

Table 2. Intervention elements included

\begin{tabular}{|c|c|c|c|}
\hline \multirow[t]{2}{*}{ Article } & \multicolumn{3}{|c|}{ Intervention elements } \\
\hline & $\begin{array}{l}\text { Information } \\
\text { provision }\end{array}$ & $\begin{array}{l}\text { Value and/or } \\
\text { preference } \\
\text { clarification } \\
\text { (explicit or implicit) }\end{array}$ & Other \\
\hline $\begin{array}{l}\text { Cupples et al. } \\
(2018)(281)\end{array}$ & Yes & Yes, explicit & $\begin{array}{l}\text { Questions regarding barriers and facilitators, goal setting, problem } \\
\text { solving, action planning, practical and emotional social support }\end{array}$ \\
\hline $\begin{array}{l}\text { Hirsch et al. (2010) } \\
(283)\end{array}$ & Yes & $\begin{array}{l}\text { Yes, ambiguous if } \\
\text { explicit or implicit }\end{array}$ & $\begin{array}{l}\text { Calculation of individual absolute risk for stroke and/or myocardial } \\
\text { infarction, exploration of subjective risk, assessment of individual risk } \\
\text { factors, risk comparison to the population with identical sex and age, } \\
\text { planning course of action }\end{array}$ \\
\hline $\begin{array}{l}\text { Hirsch et al. (2012) } \\
\text { (286) }\end{array}$ & Yes & $\begin{array}{l}\text { Yes, ambiguous if } \\
\text { explicit or implicit }\end{array}$ & $\begin{array}{l}\text { Discussion of the individual risk, discussion of treatment options and } \\
\text { plan for future actions }\end{array}$ \\
\hline $\begin{array}{l}\text { Hirsch et al. (2012) } \\
\text { (287) }\end{array}$ & Yes & $\begin{array}{l}\text { Yes, ambiguous if } \\
\text { explicit or implicit }\end{array}$ & $\begin{array}{l}\text { Discussion of the individual risk, discussion of treatment options and } \\
\text { plan for future actions }\end{array}$ \\
\hline $\begin{array}{l}\text { Hirsch et al. (2012) } \\
(288)\end{array}$ & Yes & $\begin{array}{l}\text { Yes, ambiguous if } \\
\text { explicit or implicit }\end{array}$ & $\begin{array}{l}\text { Discussion of the individual risk, discussion of treatment options and } \\
\text { plan for future actions }\end{array}$ \\
\hline $\begin{array}{l}\text { Koelewijn-van Loon } \\
\text { et al. (2008) \& } \\
\text { Koelewijn-van Loon } \\
\text { et al. (2009) (289, } \\
290)\end{array}$ & Yes & Yes, explicit & $\begin{array}{l}\text { The DA was one part of an intervention mix, the other parts being: Risk } \\
\text { assessment, graphical risk communication tool, (adapted) motivational } \\
\text { interviewing }\end{array}$ \\
\hline $\begin{array}{l}\text { Krones et al. (2010) } \\
(293)\end{array}$ & Yes & $\begin{array}{l}\text { Yes, ambiguous if } \\
\text { explicit or implicit }\end{array}$ & $\begin{array}{l}\text { Ambiguous, but in all likelihood the same as in Krones et al. (292): } \\
\text { Calculation of individual absolute risk for stroke and/or myocardial } \\
\text { infarction, exploration of subjective risk, assessment of individual risk } \\
\text { factors, risk comparison to the population with identical sex and age, } \\
\text { planning course of action }\end{array}$ \\
\hline $\begin{array}{l}\text { Sheridan et al. } \\
\text { (2013) (Protocol } \\
\text { paper) \& Keyserling } \\
\text { et al. }(2014) \text { (Effect } \\
\text { paper) }(296,298)\end{array}$ & Yes & Yes, implicit & $\begin{array}{l}\text { The DA was one part of an intervention mix and included: Calculation of } \\
\text { participants' CDH risk, showing participants how much their CHD risk } \\
\text { might be reduced by one or more of the following: Changes in diet, } \\
\text { increased PA, smoking cessation, initiation of aspirin (for men only), or } \\
\text { initiation or intensification of treatment with statins or hypertension } \\
\text { medication; encouragement to choose risk-reducing strategies, the } \\
\text { other part being: Either counselor-delivered and web-based intervention } \\
\text { sessions that included } 4 \text { intensive sessions (each up to } 60 \text { min in } \\
\text { duration depending on participants' individual pace in the web or } \\
\text { counselor-delivered sessions) at monthly intervals, followed by } 3 \\
\text { maintenance sessions (each 15-30 minutes in duration) delivered at } 2 \\
\text { month intervals, the intensive sessions included content related to } \\
\text { self-assessment of lifestyle and barriers, tips to circumvent } \\
\text { self-identified barriers, creation of first steps toward self-identified } \\
\text { actionable goals, the content of maintenance sessions was tailored } \\
\text { according to participants' success in adhering to their chosen risk } \\
\text { reducing strategy or strategies, which were assessed at the beginning of } \\
\text { the first maintenance visit. Messages focused on the following basic } \\
\text { topics: Relapse prevention, problem solving and lessons for long-term } \\
\text { maintenance, all participants received ancillary resources including a } \\
\text { cookbook, pedometers, and physical activity logs for self-monitoring of } \\
\text { exercise and an illustrated community resource guide that specified } \\
\text { local resources for healthy eating (e.g., farmers markets) and physical } \\
\text { activity (e.g., walking trails) }\end{array}$ \\
\hline
\end{tabular}


Table 2. Continued

\begin{tabular}{|c|c|c|c|}
\hline \multirow[t]{2}{*}{ Article } & \multicolumn{3}{|c|}{ Intervention elements } \\
\hline & $\begin{array}{l}\text { Information } \\
\text { provision }\end{array}$ & $\begin{array}{l}\text { Value and/or } \\
\text { preference } \\
\text { clarification } \\
\text { (explicit or implicit) }\end{array}$ & Other \\
\hline $\begin{array}{l}\text { Tinsel et al. (2017) } \\
\text { (Protocol paper) \& } \\
\text { Tinsel et al. (2018) } \\
(299,300)\end{array}$ & Yes & $\begin{array}{l}\text { Yes, ambiguous if } \\
\text { explicit or implicit }\end{array}$ & $\begin{array}{l}\text { The DAs were one part of the intervention, the other parts being: Two } \\
\text { printed booklets which contained the DAs but also self-monitoring } \\
\text { elements such as protocols; a homepage with further information about } \\
\text { cardiovascular risks and diseases and structured consultations by GPs } \\
\text { which include risk calculation (at the start and after } 4 \text { months); SDM and } \\
\text { goal setting; support individual action planning and self-monitoring. The } \\
\text { control group received everything except the brochures. }\end{array}$ \\
\hline $\begin{array}{l}\text { Van Steenkiste et } \\
\text { al. (2008) (301) }\end{array}$ & Yes & Yes, implicit & $\begin{array}{l}\text { The DA was given to patients at a first consultation after which they } \\
\text { could complete it and come back for a second consultation, the DA also } \\
\text { included: Risk charts for CVD prevention, case histories, smokers were } \\
\text { questioned about their smoking behavior, worksheet to summarize } \\
\text { patient's risk assessment, preferences for risk reduction and invitation } \\
\text { to participate in the decision-making process on personal } \\
\text { cardiovascular risk management plan }\end{array}$ \\
\hline $\begin{array}{l}\text { BinDhim et al. } \\
\text { (2014) (Protocol } \\
\text { paper) \& BinDhim } \\
\text { etl al. }(2018)(\text { Effect } \\
\text { paper) }(302,303)\end{array}$ & Yes & Yes, implicit & $\begin{array}{l}\text { Intervention group: Compulsory notification (e.g., daily motivational } \\
\text { messages), quitting diaries, (visual) quitting benefits tracker; Control } \\
\text { group: No other elements }\end{array}$ \\
\hline $\begin{array}{l}\text { Brunette et al. } \\
(2011)(304)\end{array}$ & Yes & Yes, explicit & $\begin{array}{l}\text { (Optional) tutorial on how to use a computer mouse, users could choose } \\
\text { to receive more elaborate information, video-recorded narrator who } \\
\text { identified as smoker, a smoking assessment (incl. carbon monoxide } \\
\text { meter) followed by feedback, video of a smoker that used a nicotine } \\
\text { patch during a cessation attempt, printout report that included: } \\
\text { Summary of smoking level, individual pros and cons of smoking, } \\
\text { treatment interests and a referral to a smoking cessation specialist, } \\
\text { sign-up sheet for meeting with smoking cessation specialist }\end{array}$ \\
\hline $\begin{array}{l}\text { Cupertino et al. } \\
(2010)(307)\end{array}$ & Yes & Yes, explicit & $\begin{array}{l}\text { Presentation of information in two languages (English and Spanish), } \\
\text { bilingual narrator, smoking behaviors query, combination of video and } \\
\text { audio, involvement of well-known community members, printed three } \\
\text { page tailored printout that included: Summary of reported reason for } \\
\text { quitting, level of interest in quitting, treatment preferences, personalized } \\
\text { recommendations for behavior change, for participants that were } \\
\text { interested in stopping smoking: A cessation plan, for participants that } \\
\text { were not interested in stopping smoking: Small changes to stop } \\
\text { smoking, prompt to discuss smoking cessation with a health care } \\
\text { provider, report and tips for health care providers, fax referral form for a } \\
\text { quit line, for participants that were interested in using medication: } \\
\text { Provision of nicotine patches or a coupon and prescription for } \\
\text { bupropion }\end{array}$ \\
\hline $\begin{array}{l}\text { Ferron et al. (2011) } \\
(308)\end{array}$ & $\begin{array}{l}\text { Ambiguous, but in } \\
\text { all likelihood yes }\end{array}$ & $\begin{array}{l}\text { Ambiguous, but in } \\
\text { all likelihood yes; } \\
\text { ambiguous if } \\
\text { explicit or implicit }\end{array}$ & $\begin{array}{l}\text { Ambiguous, but in all likelihood the same elements as the DA mentioned } \\
\text { in Brunette et al. ( } 304 \text { ): (Optional) tutorial on how to use a computer } \\
\text { mouse, users could choose to receive more elaborate information, } \\
\text { video-recorded narrator who identified as smoker, a smoking } \\
\text { assessment (incl. carbon monoxide meter) followed by feedback, video } \\
\text { of a smoker that used a nicotine patch during a cessation attempt, } \\
\text { printout report that included: Summary of smoking level, individual pros } \\
\text { and cons of smoking, treatment interests and a referral to a smoking } \\
\text { cessation specialist, sign-up sheet for meeting with smoking cessation } \\
\text { specialist }\end{array}$ \\
\hline
\end{tabular}


CHAPTER 6

Table 2. Continued

\begin{tabular}{|c|c|c|c|}
\hline \multirow[t]{2}{*}{ Article } & \multicolumn{3}{|c|}{ Intervention elements } \\
\hline & $\begin{array}{l}\text { Information } \\
\text { provision }\end{array}$ & $\begin{array}{l}\text { Value and/or } \\
\text { preference } \\
\text { clarification } \\
\text { (explicit or implicit) }\end{array}$ & Other \\
\hline $\begin{array}{l}\text { Hollen et al. (2013) } \\
(310)\end{array}$ & Yes & Yes, explicit & $\begin{array}{l}\text { There were five modules on: Decision making (a 17-minute video on } \\
\text { decision making in general based on Janis and Mann's conflict model of } \\
\text { decision making), smoking (a 11-minute, video on why some teens start } \\
\text { smoking and why it is hard to stop), alcohol/drug use (a 10-minute } \\
\text { videos about alcohol use), an interactive substance use module (a } 30-60 \\
\text { minute interactive practice in how to handle difficult situations with } \\
\text { substance use), and a health status module (15-minute discussion with } \\
\text { an health professional), they also provided one-on-one counseling } \\
\text { sessions, telephone calls for people with a high risk and web-based } \\
\text { support }\end{array}$ \\
\hline $\begin{array}{l}\text { McDonnell et al. } \\
\text { (2016) (313) }\end{array}$ & Yes & Yes, explicit & $\begin{array}{l}\text { The DA was one part of the intervention and included: Brief } \\
\text { decision-making tutorial (incl. a graphical handout and a CD), the other } \\
\text { parts being: Brief smoking cessation counselling by a surgeon or other } \\
\text { team member, a smoking cessation program booklet plus four } \\
\text { face-to-face sessions and up to six optional booster sessions via the } \\
\text { telephone and/or online, stress management mediation CD, and } \\
\text { medication management }\end{array}$ \\
\hline $\begin{array}{l}\text { Sheridan et al. } \\
(2010)(294)\end{array}$ & Yes & $\begin{array}{l}\text { Yes, both (tested } \\
\text { the added value of } \\
\text { an additional } \\
\text { explicit } \mathrm{VCM}^{\star} \text { ) }\end{array}$ & $\begin{array}{l}\text { Same elements as in Sheridan et al. (295), except for the tailored } \\
\text { adherence messages that were not included in this study }\end{array}$ \\
\hline $\begin{array}{l}\text { Sheridan et al. } \\
(2011)(295)\end{array}$ & Yes & Yes, explicit & $\begin{array}{l}\text { The DA was one part of the intervention and included: Calculation of } \\
\text { patients' overall risk of CHD events in the next } 10 \text { years, encouragement } \\
\text { to choose risk-reducing strategies, and coaching to communicate their } \\
\text { decisions with their physicians for this audio clips about ways to } \\
\text { overcome common communication barriers were provided, the other } \\
\text { part being: Tailored adherence messages to help patients to circumvent } \\
\text { self-identified barriers and gain the resources and skills for adherence }\end{array}$ \\
\hline $\begin{array}{l}\text { Sheridan et al. } \\
\text { (2014) (297) }\end{array}$ & Yes & Yes, explicit & $\begin{array}{l}\text { The DA was one part of the intervention and included: Calculation of } \\
\text { patients' overall risk of CHD events in the next } 10 \text { years, encouragement } \\
\text { to choose risk-reducing strategies, and coaching to communicate their } \\
\text { decisions with their physicians for this audio clips about ways to } \\
\text { overcome common communication barriers were provided and a } \\
\text { summary of their DA session to initiate discussion with their provider, } \\
\text { the other part being: Tailored adherence messages to help patients to } \\
\text { circumvent self-identified barriers and gain the resources and skills for } \\
\text { adherence }\end{array}$ \\
\hline $\begin{array}{l}\text { Warner et al. (2015) } \\
\text { (315) }\end{array}$ & Yes & Yes, implicit & $\begin{array}{l}\text { Simple graphic illustrating the effects of smoking on the body, and a } \\
\text { motivational phrase }\end{array}$ \\
\hline
\end{tabular}

Note. Articles are sorted thematically, alphabetically, and chronologically. Ambiguous information was not verified with the original authors. *Called a value clarification exercise (VCE) in their article 


\subsubsection{Theoretical foundations}

Twenty-two studies (71\%) (281-283, 285, 286, 292-295, 297, 299-306, 309-314) reported using theoretical frameworks, most commonly to identify relevant outcome measures $(n=15$, $48 \%)(283,285,286,292-295,297,301-305,309,310,314)$. Janis' and Mann's conflict theory of decision making was used most often $(n=6,19 \%)(294,297,310,312-314)$ - however, largely the same researchers were involved. An overview over the theoretical foundations can be seen in table 3 .

\subsubsection{Effectiveness and cost-effectiveness of the identified DAs}

Most effects where tested in either a cluster $(n=7,23 \%)(283,284,289-291,311)$ or a randomized controlled trial $(n=8,26 \%)(294,295,297,302,303,305,309,310,314)$. In this result section null effects are defined as insignificant findings that reflect neither an increase nor a decrease.

Table 3. Use of theories

\begin{tabular}{lll}
\hline Has a theory been used at all? & Yes & $n=22$ \\
& Not reported & $n=9$
\end{tabular}

Note. *In some studies, multiple theories have been used for multiple purposes. Therefore, the absolute amount exceeds 31 . 


\section{CHAPTER 6}

\subsubsection{Effects on the attributes of the choice made}

In six studies, knowledge (19\%) was assessed $(292,297,300,303,305,315)$, but only one (297) reported a significant increase in knowledge as compared to baseline measurement. In three studies (10\%), null effects were reported regarding knowledge $(292,305,315)$. All other studies examined knowledge only as part of another overarching concept $(300,303)$, e.g., informed choice. Effects on risk perception were examined in two studies $(6 \%)(291$, 297), both found an increase in appropriateness of risk perceptions, however in one study the effects disappeared after correction for baseline characteristics (291) and in the other effects were not compared to a control group (297). Value-congruency was tested in four studies $(13 \%)(293,294,297,303)$. In one of those studies value-congruency was not examined in isolation (303). Sheridan et al. (294) found that adding an explicit VCM (called a VCE in their article) did not increase value-consistency. In one study an increased attitude towards the chosen option (i.e., "actual" value-consistency) was reported that was compared to a control group (293), while in another study positive effects on perceived valueconsistency that were not compared to a control group were reported (297). The one article that reported on the measurement of regret reported a significant positive effect (i.e., a decrease in regret) (292).

\subsubsection{Effects on the attributes of the decision-making process}

The most commonly investigated attribute was patient-practitioner communication $(\mathrm{n}=12$, $39 \%)(284-286,291,297,301,304,305,307,309,312,315)$. However, mixed effects were found: Decrease in communication $(n=1)(307)$, increase in communication $(n=2,6 \%)(297$, 304), increase in satisfaction with the communication $(n=1)(291)$, and null effects $(n=1)$ (315). Other studies mainly reported descriptive characteristics, e.g., that most of the exposed patients were satisfied (285). In four (13\%) out of seven (23\%) studies in which participation in decision making was investigated positive effects compared to a control group were found $(283,285,292,315)$ (the majority came from similar researchers), in one study null effects were reported (297). Positive effects were found regarding decisional conflict, assessed in four studies $(13 \%)(294,297,303,315)$; null effects were only reported in one study (294) on the added value of an explicit VCM. Positive effects were both observed compared to a control group $(n=2,6 \%)(303,315)$, and not compared to a control group $(n=1)(297)$. While the proportion of undecided people was reported in six studies $(19 \%)(281,285-287,293,315)$ (again, the majority came from similar researchers), only in one the effect was tested (293). They found a positive effect compared to a control group (293). No study reported effects on decisional satisfaction.

\subsubsection{Effects on behavior}

In $18(58 \%)$ articles an assessment on the impact of the DA on behavior was reported (281, $282,285,286,290,291,298,300,303-307,309,311,312,314,315)$. 


\subsection{Dietary behavior}

In one study in which differences between two study groups were tested, positive effects due to the interventions on fat and vegetable intake were found (290). However, effects on fat and vegetable intake were not replicated in multilevel analyses (290). In another study with a control group (same researchers) null effects for fat, fruit and vegetable consumption were found (291), while in another study negative effects on overall diet were reported (300). In one study mixed effects in terms of fruit and vegetable intake were reported (282) which were not compared to a control group. In this study two different versions of a DA were tested: One targeting PA, the other fruit and vegetable intake (282). Interestingly, only the version targeting PA resulted in an increase in fruit and vegetable consumption, the fruit and vegetable version resulted in a small decrease in fruit and vegetable consumption (282). In another study the same DA was compared alongside counseling or a web-based lifestyle intervention (thus, both study arms received the same DA): Positive effects were found for fat quality, fruit, and vegetable intake (298). One article simply reported that diet changed without further details (281).

\subsection{Physical activity (PA)}

In two of the three studies $(6 \%)(290,291)$ comparing effects to a control group no effects on PA were found (same researchers), the one that did (300) was a pilot study that only reported descriptive analyses. Within the study that tested two different versions of the same DA (one for PA, one for fruit and vegetables): Positive effects regarding PA were found in both groups (282). Strikingly, the effects were stronger in the non-PA version. In another study without control group positive effects on weekly PA time and sedentary behavior were found (281), negative effects were found for minutes of PA and daily number of steps (281). The study that compared the effects of the DA alongside counseling or a web-based lifestyle intervention found positive effects for weekly walking time and daily number of steps. However, the effect for weekly walking time was only observed in the counselor group (298).

\subsection{Substance use}

In studies including a control group positive effects on smoking cessation aid uptake $(n=3$, $10 \%)(303,304,306)$ and smoking abstinence $(n=1)(303)$ were found, while null effects were found on perioperative smoking behavior $(n=1)(315)$, smoking cessation medication $(n=1)(311)$, smoking abstinence $(n=3,10 \%)(290,291,311)$ and smoking, alcohol and illicit drug uptake $(n=1)$ (314). Only in one study that included a control group negative effects regarding smoking were found, however positive effects on alcohol consumption were found as well (300). Interestingly, in one study both an effect on smoking cessation aid uptake and abstinence was found, but they researchers did not find that the DA's effect on abstinence was mediated by the quitting method (303). In another study (290) a difference 


\section{CHAPTER 6}

between intervention and control group was found, however the difference was already present at baseline. In the one study without control group, positive effects were found on smoking cessation aid uptake and number of cigarettes, while negative effects were found on planning of a quit date and talking to health-care providers about smoking cessation (307). Other effects that were found: Adding carbon monoxide feedback to a DA did not make it more effective $(n=1)(305)$, a DA for dyads (patient plus family member) seemed to be more effective for patients' quitting behavior than family members' quitting behavior $(n=1)(312)$ and in the study (298) that compared the effects of the DA alongside counseling or a web-based lifestyle intervention positive effects for smoking were found in both groups.

\subsubsection{Effects on adherence to the chosen option}

Adherence was assessed in four (13\%) studies $(281,295,303,315)$. Three (10\%) compared the effects to a control group; one reported null (315), one positive effects (i.e., increased adherence) (295), and one reported that $97.7 \%$ adhered to their chosen option regardless of the assigned group (303).

\subsubsection{Effects on economic impact}

Cost-effectiveness was assessed in one study, however not the cost-effectiveness of the DA itself was tested but rather of a counseling or a web-based intervention used next to the DA (298).

\subsubsection{Effects on health outcomes}

Health status was assessed in five studies $(16 \%)(290,292,295,298,300)$, both null $(n=3$, $10 \%)(290,292,300)$ and positive effects $(n=2,6 \%)(295,298)$ (both from similar research teams) were found. Quality of life (298) and anxiety (291) were only assessed once, in both cases significant improvements were found. No study reported effects on depression and emotional distress.

\subsection{Results grey literature}

The initial search into the DALI resulted in 10 DAs (dietary behavior $n=5$ and substance use $n=5$ ). Only four DAs were still available online at the time of the search (317-320). All DAs stemmed from the same developer (www.healthwise.org), a nonprofit organization aimed at providing digital health education. All DAs shared a similar design. Theory application was not described.

All DAs made use of information provision and explicit elements to clarify values and preferences. Other elements were personal stories, a knowledge quiz, and a summary. Duration to complete the DAs was not reported.

The DAs were not reported in any scientific publications. No effects were reported. An overview of currently online accessible DAs can be seen in table 4 . 
Table 4. DA characteristics grey literature

\begin{tabular}{lll}
\hline Name & Behavior (general) & Behavior (specially) \\
\hline $\begin{array}{l}\text { Healthwise: Quitting Smoking: } \\
\text { Should I Use Medicine? (317) } \\
\text { Healthwise: Obesity: }\end{array}$ & Substance use & Smoking (cessation) \\
$\begin{array}{l}\text { Should I Use a Diet Plan to Lose Weight? (318) } \\
\text { Healthwise: Weight Management: }\end{array}$ & Dietary behavior & Diet \\
$\begin{array}{l}\text { Should I Use Over-the-Counter Diet Aids? (319) } \\
\text { Healthwise: Sleep Apnea: }\end{array}$ & Dietary behavior & Use of diet aids \\
Should I Have a Sleep Study? (320) & Sleep-related behaviors & General sleep management \\
\hline
\end{tabular}

\section{DISCUSSION AND CONCLUSION}

\subsection{Discussion}

With this scoping review we aimed to synthesize the literature on DAs that focus on preventive health-related behaviors by reviewing available information regarding their characteristics, intervention elements, theoretical foundations and (cost-)effectiveness. We identified 35 scientific papers describing DA development and/or evaluation and four DAs that focus on preventive health-related behaviors in the grey literature. We will focus on three key areas in this discussion: (1 Characteristics and intervention elements of identified DAs, ( 2 theoretical foundations of the identified DAs, and ( 3 effectiveness of the identified DAs.

\subsubsection{Characteristics and intervention elements of the identified DAs}

Identified DAs focused most often on substance use, primarily smoking. This could be due to the fact that smoking cessation trajectories show similarities with clinical treatment and screening trajectories, which is where the majority of DAs traditionally have been applied (127). For example, one of the options that is regularly named in smoking cessation DAs is pharmacological support (e.g.. (303)).

DAs were often combined with additional intervention elements. Therefore, it was difficult to ascertain the impact of the DA independent from these other components, as the additional components often had their basis in behavioral change theories, rather than informed decision making. Consequently, tested outcomes varied widely among studies, limiting the current evidence base for any behavior- or decision-related outcome.

Future studies should examine which intervention elements are effective regarding informed decision making in the area of preventive health-related behaviors. Furthermore, studies should be conducted to disentangle which intervention elements can be deployed to support which processes. To this end, however, consensus should be reached on which outcomes are relevant to be tested in studies investigating DAs that focus on preventive health-related behaviors. This would not only allow different intervention elements to be tested using the same criteria but would also enable developers of DAs that focus on preventive health-related behaviors to develop DAs that are even more rooted in evidence 


\section{CHAPTER 6}

than current DAs. Ultimately, this could result in a taxonomy as used in behavior change (321) which clearly describes the purpose of most often applied intervention elements. Theoretical work to understand VCMs' effects and how those effects can be accomplished have recently been undertaken $(271,322)$.

\subsubsection{Theoretical foundations of the identified DAs}

Around $70 \%$ of the studies reported that they used a theory, most commonly to identify relevant outcome measures. Multiple studies used theories such as the Self-Determination Theory (323) or the Theory of Planned Behavior (112); theories meant to explore motivation or behavior (change). We also found studies that used decision-making-focused theories, such as the Conflict Theory of Decision Making (122), however these are not explicitly designed to support people in changing behavior. Given the dual purpose of DAs that focus on preventive health-related behaviors, insights from multiple theories should be used to develop these DAs.

There are two possible approaches to integrate insights from both areas when developing DAs that focus on preventive health-related behaviors: (1 Developers could flexibly integrate insights from multiple theories on respectively behavioral change and informed decision making as proposed by Peters \& Crutzen (324), (2 or attempts could be made to establish an integrative framework that can be applied in multiple (unrelated) DA development projects. The second approach could be particularly helpful for developers that are not familiar with both research fields.

\subsubsection{Effectiveness of the identified DAs}

Studies reported positive effects such as uptake of effective smoking cessation aids and smoking abstinence, however interpretation is somewhat difficult as not all studies followed an RCT protocol and as we could not synthesize the effects quantitatively. Also, a formal analysis of the quality of the evidence has not taken place in this scoping review as this form of knowledge synthesis (often) does not include quality assessments in the same form as systematic reviews (276). However, our findings are in line with a systematic review (270) in which it was found that smoking cessation DAs can be effective, but that there was major heterogeneity within studies and DAs. Beneficial effects were also identified regarding PA and nutritional behavior, however, due to the relatively low numbers of studies and the mixed findings found in the included studies, no clear conclusions can be drawn at this time.

Interestingly, the majority of the identified studies failed to report effects on decisional outcomes. Future studies should investigate how DAs that focus on preventive healthrelated behaviors affect those decisional outcomes as well and how these outcomes relate to behavior (change). Insights from Self-Determination Theory (323), for example, would suggest that the offering of choices (i.e., what DAs do inherently) can support individuals in becoming autonomously motivated towards self-chosen options, which in turn can lead to greater behavioral maintenance $(325,326)$. 


\subsection{Limitations}

A possible limitation was the focus on studies as the units of analysis rather than individual DAs. However, not all studies that referred to similar DAs clearly described how they related to one another, which made it impossible to report results per DA. To minimize the impact of this on our results, we highlighted if studies were conducted by the similar author(s). Another possible limitation would be that we decided to exclude all treatment DAs, including those aimed at preventing secondary diseases or complications (e.g., cardiovascular disease due to diabetes mellitus). However, our working definition of DAs that focus on preventive health-related behaviors has only focused on primary disease prevention and we are convinced that DAs aimed at primary, secondary and tertiary prevention should be explored separately. Hence, the focus on DAs that focus on primary prevention.

\subsection{Future research directions}

Based on the discussion above, we have identified three main areas of interest for further research: (1 Establishing which intervention elements are effective regarding decision making in the domain of preventive health-related behaviors, and for which processes, (2 strengthening the integration between theoretical insights from behavior change and informed decision making, by either adopting a flexible approach or by establishing an integrative framework, and (3 conducting more randomized trials to enable systematic reviews and meta-analyses in order to draw stronger conclusions regarding behavioral and decisional outcomes and how those relate to one another.

\subsection{Practice Implications}

While scoping reviews do not allow for strong conclusions to be drawn (compared to other forms of knowledge syntheses), our results show that DAs can potentially be beneficial in supporting people to change preventive health-related behaviors - especially regarding smoking (particularly when taken together with other evidence (270). As such, DAs might be one potential approach to counteract the rise of noncommunicable diseases. However, further research is needed to substantiate this.

\subsection{Conclusions}

This study was the first attempt to broadly synthesize knowledge regarding DAs aimed at preventive health-related behavioral decisions. Findings regarding the effects on behavior were potentially promising, especially regarding smoking (particularly when taken together with other evidence (270)). However, while certain beneficial effects could be identified, interpretation was hindered by heterogenous reporting. Certain areas of improvement were identified, such as establishing which intervention elements are effective regarding decision making in the domain of preventive health-related behaviors. 


\section{APPENDIX A - PREFERRED REPORTING ITEMS FOR SYSTEMATIC REVIEWS AND META-ANALYSES EXTENSION FOR SCOPING REVIEWS (PRISMA-SCR) CHECKLIST}

Table A.1. PRISMA-ScR Checklist

\begin{tabular}{|c|c|c|c|}
\hline Section & Item & PRISMA-ScR Checklist item & $\begin{array}{l}\text { REPORTED ON } \\
\text { PAGE \# }\end{array}$ \\
\hline \multicolumn{4}{|l|}{ Title } \\
\hline Title & 1 & Identify the report as a scoping review. & 106/Title page \\
\hline \multicolumn{4}{|l|}{ Abstract } \\
\hline $\begin{array}{l}\text { Structured } \\
\text { Summary }\end{array}$ & 2 & $\begin{array}{l}\text { Provide a structured summary that includes (as applicable): } \\
\text { background, objectives, eligibility criteria, sources of evidence, } \\
\text { charting methods, results, and conclusions that relate to the } \\
\text { review questions and objectives. }\end{array}$ & 107/Abstract \\
\hline \multicolumn{4}{|l|}{ Introduction } \\
\hline Rationale & 3 & $\begin{array}{l}\text { Describe the rationale for the review in the context of what is } \\
\text { already known. Explain why the review questions/objectives } \\
\text { lend themselves to a scoping review approach. }\end{array}$ & 108-109 \\
\hline Objectives & 4 & $\begin{array}{l}\text { Provide an explicit statement of the questions and objectives } \\
\text { being addressed with reference to their key elements (e.g., } \\
\text { population or participants, concepts, and context) or other } \\
\text { relevant key elements used to conceptualize the review } \\
\text { questions and/or objectives. }\end{array}$ & 109 \\
\hline \multicolumn{4}{|l|}{ Methods } \\
\hline $\begin{array}{l}\text { Protocol and } \\
\text { Registration }\end{array}$ & 5 & $\begin{array}{l}\text { Indicate whether a review protocol exists; state if and where it } \\
\text { can be accessed (e.g., a Web address); and if available, } \\
\text { provide registration information, including the registration } \\
\text { number. }\end{array}$ & 109 \\
\hline Eligibility criteria & 6 & $\begin{array}{l}\text { Specify characteristics of the sources of evidence used as } \\
\text { eligibility criteria (e.g., years considered, language, and } \\
\text { publication status), and provide a rationale. }\end{array}$ & 110 \\
\hline Information sources & 7 & $\begin{array}{l}\text { Describe all information sources in the search (e.g., } \\
\text { databases with dates of coverage and contact with authors to } \\
\text { identify additional sources), as well as the date the most } \\
\text { recent search was executed. }\end{array}$ & $109-110$ \\
\hline Search & 8 & $\begin{array}{l}\text { Present the full electronic search strategy for at least } 1 \\
\text { database, including any limits used, such that it could be } \\
\text { repeated. }\end{array}$ & $\begin{array}{l}\text { 109-110, } \\
\text { appendix B }\end{array}$ \\
\hline $\begin{array}{l}\text { Selection of sources } \\
\text { of evidence }\end{array}$ & 9 & $\begin{array}{l}\text { State the process for selecting sources of evidence (i.e., } \\
\text { screening and eligibility) included in the scoping review. }\end{array}$ & $110-111$ \\
\hline $\begin{array}{l}\text { Data charting } \\
\text { Process }\end{array}$ & 10 & $\begin{array}{l}\text { Describe the methods of charting data from the included } \\
\text { sources of evidence (e.g., calibrated forms or forms that have } \\
\text { been tested by the team before their use, and whether data } \\
\text { charting was done independently or in duplicate) and any } \\
\text { processes for obtaining and confirming data from } \\
\text { investigators. }\end{array}$ & $\begin{array}{l}\text { 110-111, } \\
\text { appendix C }\end{array}$ \\
\hline Data items & 11 & $\begin{array}{l}\text { List and define all variables for which data were sought and } \\
\text { any assumptions and simplifications made. }\end{array}$ & Appendix C \\
\hline
\end{tabular}


Table A.1. Continued

\begin{tabular}{|c|c|c|c|}
\hline Section & Item & PRISMA-ScR Checklist item & $\begin{array}{l}\text { REPORTED ON } \\
\text { PAGE \# }\end{array}$ \\
\hline $\begin{array}{l}\text { Critical appraisal of } \\
\text { individual sources of } \\
\text { evidence }\end{array}$ & 12 & $\begin{array}{l}\text { If done, provide a rationale for conducting a critical appraisal } \\
\text { of included sources of evidence; describe the methods used } \\
\text { and how this information was used in any data synthesis (if } \\
\text { appropriate). }\end{array}$ & Not applicable \\
\hline Synthesis of results & 13 & $\begin{array}{l}\text { Describe the methods of handling and summarizing the data } \\
\text { that were charted. }\end{array}$ & 109 \\
\hline \multicolumn{4}{|l|}{ Results } \\
\hline $\begin{array}{l}\text { Selection of } \\
\text { Sources of evidence }\end{array}$ & 14 & $\begin{array}{l}\text { Give numbers of sources of evidence screened, assessed for } \\
\text { eligibility, and included in the review, with reasons for } \\
\text { exclusions at each stage, ideally using a flow diagram. }\end{array}$ & Figure 1 \\
\hline $\begin{array}{l}\text { Characteristics of } \\
\text { sources of } \\
\text { Evidence }\end{array}$ & 15 & $\begin{array}{l}\text { For each source of evidence, present characteristics for which } \\
\text { data were charted and provide the citations. }\end{array}$ & $111-130$ \\
\hline $\begin{array}{l}\text { Critical appraisal } \\
\text { within sources of } \\
\text { evidence }\end{array}$ & 16 & $\begin{array}{l}\text { If done, present data on critical appraisal of included sources } \\
\text { of evidence (see item 12). }\end{array}$ & Not applicable \\
\hline $\begin{array}{l}\text { Results of } \\
\text { Individual sources of } \\
\text { evidence }\end{array}$ & 17 & $\begin{array}{l}\text { For each included source of evidence, present the relevant } \\
\text { data that were charted that relate to the review } \\
\text { questions and objectives. }\end{array}$ & $111-130$ \\
\hline Synthesis of results & 18 & $\begin{array}{l}\text { Summarize and/or present the charting results as they relate } \\
\text { to the review questions and objectives. }\end{array}$ & $111-130$ \\
\hline \multicolumn{4}{|l|}{ Discussion } \\
\hline $\begin{array}{l}\text { Summary of } \\
\text { Evidence }\end{array}$ & 19 & $\begin{array}{l}\text { Summarize the main results (including an overview of } \\
\text { concepts, themes, and types of evidence available), link to the } \\
\text { review questions and objectives, and consider the relevance } \\
\text { to key groups. }\end{array}$ & $130-132$ \\
\hline Limitations & 20 & Discuss the limitations of the scoping review process. & 132 \\
\hline Conclusions & 21 & $\begin{array}{l}\text { Provide a general interpretation of the results with respect to } \\
\text { the review questions and objectives, as well as potential } \\
\text { implications and/or next steps. }\end{array}$ & 133 \\
\hline \multicolumn{4}{|l|}{ Funding } \\
\hline Funding & 22 & $\begin{array}{l}\text { Describe sources of funding for the included sources of } \\
\text { evidence, as well as sources of funding for the scoping } \\
\text { review. Describe the role of the funders of the scoping review. }\end{array}$ & $\begin{array}{l}\text { 106/Title page, } \\
\text { Table } 1\end{array}$ \\
\hline
\end{tabular}




\section{APPENDIX B - SEARCH STRINGS}

Table B.1. Search Strings for PubMed

\begin{tabular}{|c|c|}
\hline Behavior & Search terms \\
\hline $\begin{array}{l}\text { Dietary } \\
\text { behavior }\end{array}$ & 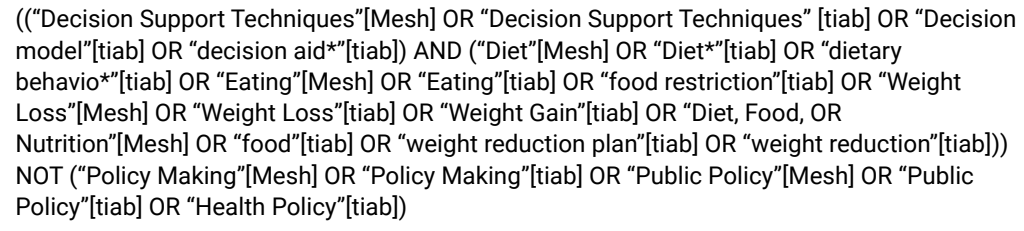 \\
\hline $\begin{array}{l}\text { Physical } \\
\text { activity }\end{array}$ & 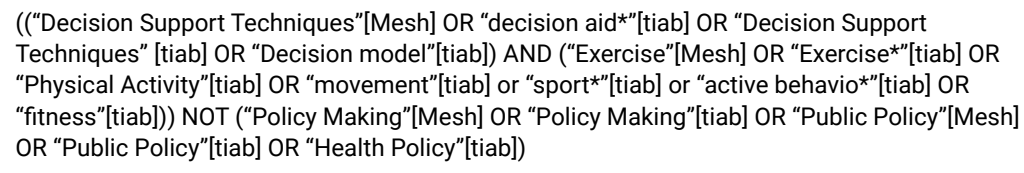 \\
\hline $\begin{array}{l}\text { Sleep-related } \\
\text { behaviors }\end{array}$ & 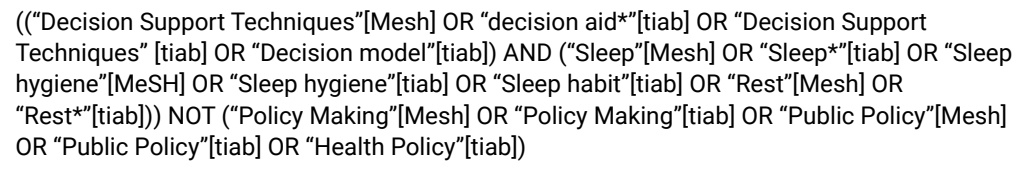 \\
\hline Substance use & 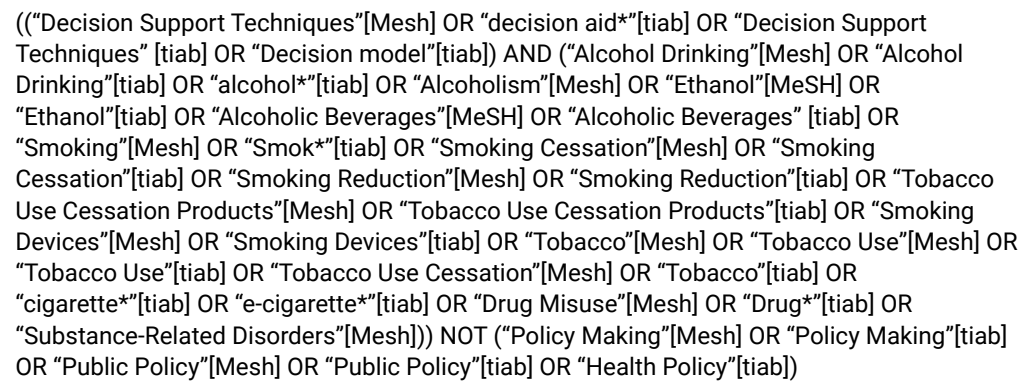 \\
\hline
\end{tabular}


Table B.2. Search Strings for PsycINFO and CINAHL

\begin{tabular}{|c|c|}
\hline Behavior & Search terms \\
\hline $\begin{array}{l}\text { Dietary } \\
\text { behavior }\end{array}$ & 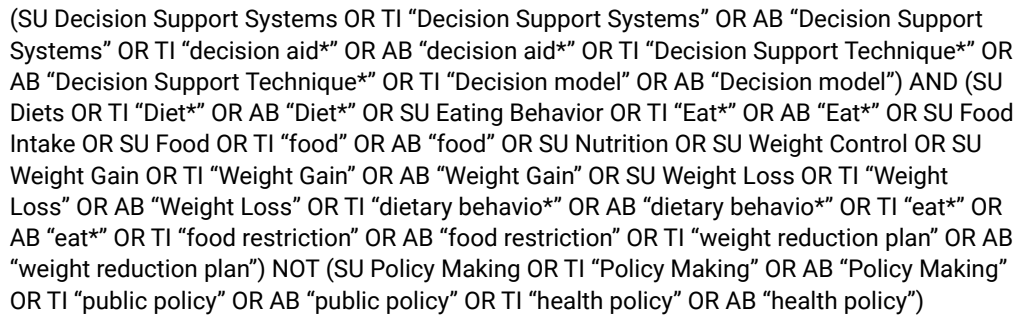 \\
\hline $\begin{array}{l}\text { Physical } \\
\text { activity }\end{array}$ & 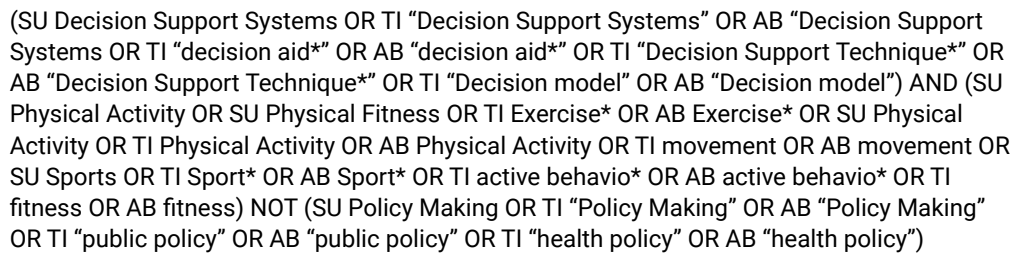 \\
\hline $\begin{array}{l}\text { Sleep-related } \\
\text { behaviors }\end{array}$ & 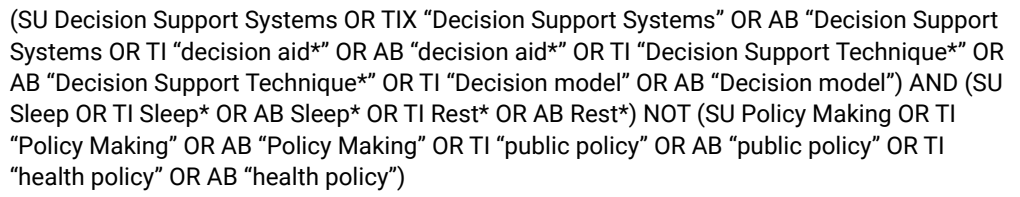 \\
\hline Substance use & 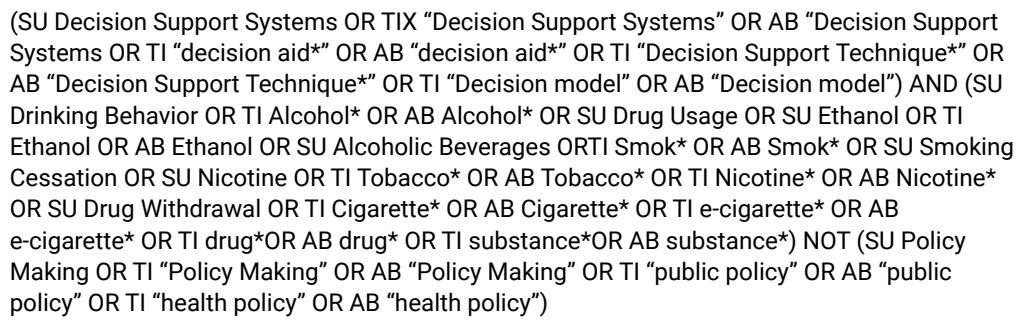 \\
\hline
\end{tabular}

Table B.3. Search Strings for Google Scholar

\begin{tabular}{|c|c|}
\hline Behavior & Search terms \\
\hline $\begin{array}{l}\text { Dietary } \\
\text { behavior }\end{array}$ & $\begin{array}{l}\text { ((“Decision Support Techniques” OR “Decision model” OR “decision aid*”) AND (“Diet*” OR } \\
\text { "Eat* OR “food*” OR “Weight*”)) -policy }\end{array}$ \\
\hline $\begin{array}{l}\text { Physical } \\
\text { activity }\end{array}$ & $\begin{array}{l}\text { (("Decision Support Techniques" OR “Decision model" OR "decision aid*”) AND (“Exercise*” } \\
\text { OR "Physical Activity" OR "move*” or "sport*” or "active behavio*" OR "fitness")) -policy }\end{array}$ \\
\hline $\begin{array}{l}\text { Sleep-related } \\
\text { behaviors }\end{array}$ & $\begin{array}{l}\text { ((“Decision Support Techniques" OR “Decision model” OR "decision aid*”) AND (“Sleep*” OR } \\
\text { "Rest*”)) -policy }\end{array}$ \\
\hline Substance use & 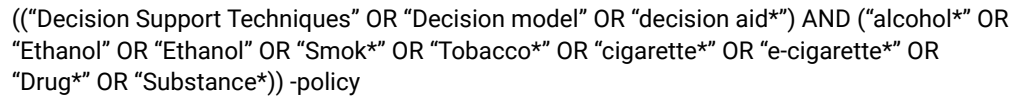 \\
\hline
\end{tabular}




\section{APPENDIX C - EXTRACTED INFORMATION}

Table C.1. Extracted information

\begin{tabular}{|c|c|}
\hline Questions & Sub questions \\
\hline \multicolumn{2}{|l|}{$\begin{array}{l}\text { On which behavior did the DA (under study) focus, } \\
\text { both in general (e.g., dietary behavior) and specifically } \\
\text { (e.g., weight loss)? }\end{array}$} \\
\hline \multicolumn{2}{|l|}{ How was the (studied) DA delivered to the user? } \\
\hline \multicolumn{2}{|l|}{ How long did it take to complete the DA (under study)? } \\
\hline Of which elements did the DA (under study) consist? & $\begin{array}{l}\text { - Did the DA contain information provision elements? } \\
\text { Did the DA contain elements to clarify values and } \\
\text { preferences? } \\
\text { - Were those elements explicit or implicit? } \\
\text { - Which other intervention elements were employed? }\end{array}$ \\
\hline $\begin{array}{l}\text { Was the DA (under study) scientifically published (and } \\
\text { was certain necessary information described)? }\end{array}$ & $\begin{array}{l}\text { - If it was, what was/were the: } \\
\text { o study design and methodology? } \\
\text { o study population? } \\
\text { o aims/purposes? } \\
\text { o origin/country of origin? } \\
\text { o author(s)? } \\
\text { o year of publication? }\end{array}$ \\
\hline How were theories used? & $\begin{array}{l}\text { - Specifically: } \\
\text { o Has a theory been used at all? } \\
\text { o From which field does the theory come? } \\
\text { o Which theory has been used specifically and how } \\
\text { was it used? } \\
\text { o Was there an impact on the outcomes through the } \\
\text { use of theory? }\end{array}$ \\
\hline $\begin{array}{l}\text { What were the effects on the attributes of the choice } \\
\text { made? }\end{array}$ & $\begin{array}{l}\text { - Specifically, the effects on: } \\
\text { o knowledge? } \\
\text { o accurate risk perceptions? } \\
\text { o value congruency? } \\
\text { o regret? }\end{array}$ \\
\hline $\begin{array}{l}\text { What were the effects on the attributes of the } \\
\text { decision-making process? }\end{array}$ & $\begin{array}{l}\text { - Specifically, the effects on: } \\
\text { o decisional conflict? } \\
\text { o proportion undecided? } \\
\text { o decisional satisfaction? } \\
\text { o patient-practitioner communication, if applicable? } \\
\text { o participation in decision making, if applicable? }\end{array}$ \\
\hline What were the effects on behavior? & $\begin{array}{l}\text { - Specifically, the effects on: } \\
\text { o (actual) behavior after the choice has been made? } \\
\text { o adherence to chosen option (time of adherence)? }\end{array}$ \\
\hline What were the results regarding economic impact? & $\begin{array}{l}\text { - Specifically, the effects on: } \\
\text { o costs? } \\
\text { o cost effectiveness? }\end{array}$ \\
\hline What were the effects on health outcomes? & $\begin{array}{l}\text { - Specifically, the effects on: } \\
\text { o health status? } \\
\text { o quality of life? } \\
\text { o anxiety? } \\
\text { o depression? } \\
\text { o emotional distress? }\end{array}$ \\
\hline
\end{tabular}


Scoping review 


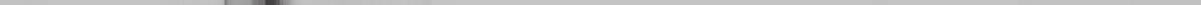




\section{CHAPTER 7}

The usability of an online tool to promote the use of evidence-based smoking cessation interventions

This chapter has been published as:

Zijlstra, D. N., Bolman, C. A., Muris, J. W., \& de Vries, H. (2021). The Usability of an Online Tool to Promote the Use of Evidence-Based Smoking Cessation Interventions. International Journal of Environmental Research and Public Health, 18(20), 10836. https://doi.org/10.3390/ijerph182010836 


\section{ABSTRACT}

Introduction: To increase usage of evidence-based smoking cessation interventions (EBSCIs) among smokers, an online decision aid (DA) was developed. The aims of this study were 1 ) to conduct a usability evaluation; 2 ) to conduct a program evaluation and evaluate decisional conflict after using the DA and 3) to determine the possible change in the intention to use EBSCls before and directly after reviewing the DA.

Methods: A cross-sectional study was carried out in September 2020 by recruiting smokers via the internet $(\mathrm{N}=497)$. $T$-tests and chi-square tests were conducted to test the differences between smokers who differed in perceived usability of the DA on the program evaluation and in decisional conflict. The possible changes in intention to use EBSCls during a cessation attempt before and after reviewing the DA were tested using t-tests, the McNemar test and $\chi^{2}$ analysis.

Results: Participants evaluated the usability of the DA as moderate (MU; $n=393$, $79.1 \%$ ) or good (GU; $n=104,20.9 \%$ ) GU smokers rated higher on all elements of the program evaluation and experienced less decisional conflict but also displayed a higher intention to quit. After reviewing the DA, participants on average had a significantly higher intention to use more EBSCls, in particular in the form of eHealth.

Conclusions: Recommendations to make the DA more usable could include tailoring, using video-based information and including value clarification methods. Furthermore, a hybrid variant in which smokers can use the DA independently and with the guidance of a primary care professional could aid both groups in choosing a fitting EBSCI option. 


\section{INTRODUCTION}

With eight million deaths occurring worldwide as a result of several types of cancer, cardiovascular diseases and respiratory diseases (6), smoking is the most important cause of preventable death $(6,10)$. In the Netherlands alone, this represents approximately 20,000 mortality cases (11) but also results in other losses for society, such as work loss because of illness or absence and higher healthcare costs (12). Beyond added costs, smoking is also one of the factors responsible for greater inequality between high socioeconomic status (SES) and low-SES households (13), as people from low-SES households are more likely to smoke but have fewer material and social resources (327). Therefore, a decrease in smoking prevalence is a significant goal for the Dutch public health domain (22).

Currently, approximately $20.2 \%$ of the Dutch population smokes (9). Among lesseducated groups, this percentage is higher, at $23.9 \%$ (9). In 2020, $35.6 \%$ of all Dutch smokers made a serious attempt at quitting (9). However, only 3-5\% of smokers who attempt to quit without help succeed in their first effort (137), and, on average, as many as 30 or more quit attempts may be required before smokers are successful for longer than 12 months (138). To help smokers in their smoking cessation attempts, several evidence-based smoking cessation interventions (EBSCls) have been developed. EBSCls are proven to double the likelihood of successful smoking cessation (86).

There are two principal forms of EBSCls: behavioral and pharmacological support. Behavioral support can include face-to-face counseling by a healthcare professional (HCP) in the GP setting, such as a general practitioner (GP) or a practice nurse (PN). Other forms of behavioral support outside the GP setting include counseling by a trained stop coach outside the GP-setting $(44,57,62-65)$, tailored online counseling known as eHealth (69, 178), telephone counseling (179) and group counseling (74). Effectiveness rates of behavioral support range between one and three percent for very brief advice on quitting $(57,242)$ by a GP and seven to 14 percent for more extensive forms of counseling, in contrast to unassisted quit attempts $(73,74)$. Pharmacological support includes nicotine replacement therapy (NRT; e.g., nicotine gum or patches) and pharmacotherapy. NRT has an effectiveness rate of $50-60 \%$, if correctly used, in comparison with no treatment or a placebo (79). For pharmacotherapy, the effectiveness rate ranges from 55 to $77 \%$ in comparison with no treatment or a placebo $(80,81)$. A combination of behavioral and pharmacological support is recommended and required when the smoker wants to be entitled to reimbursement from a health insurer $(22,52,53)$. In addition to EBSCls, there is also non-evidence-based cessation assistance, for which no firm evidence base has yet been found. Examples of non-evidence-based cessation assistance include acupuncture, laser therapy and electrostimulation (85). These options are not usually reimbursed by health insurers. Providing smokers with information and guidance to help them decide which $\mathrm{EBSCl}$ would best fit their needs and preferences might increase their involvement 
in and commitment to their own treatment processes $(103,104)$. However, only $25-30 \%$ of smokers report having used behavioral counseling methods $(88,89)$.

Reaching smokers, motivating them to quit and educating them on EBSCls are difficult to achieve. The primary care setting (PCS) offers an entry point for reaching smokers, as most people who smoke visit their PCS yearly, often for related complaints such as asthma and $\operatorname{COPD}(39,96,328)$. Within the PCS, practice nurses (PNs) provide the majority of smoking cessation counseling (182), based on the Dutch guideline for smoking cessation care (DGSCC) $(52,53)$. This guideline is based on an evidence-based counseling method, the minimal intervention smoking cessation strategy (MIS) (44), which is similar to the internationally-used $5 \mathrm{~A}$ protocol of ask, advise, asses, assist, and arrange (177). However, PNs do not always adhere to these guidelines, particularly the assist and arrange aspects, in which they are asked to provide smoking cessation counseling or refer smokers to other EBSCIs. This might be due to a lack of knowledge or low self-efficacy related to helping their patients make informed decisions $(44,52,225)$. An overview of effective evidencebased smoking cessation tools might help counselors and smokers decide on the most preferred cessation method (127).

The usability of such an overview among PNs and smoking patients willing to quit smoking has been explored in earlier research, revealing a high appreciation for but low uptake of the materials $(228,253)$. However, due to a low recruitment rate during the present randomized controlled trial, which evaluated interventions among smokers recruited via the PCS (253), this study retested the usability of the materials among a larger group of smokers to explore whether the materials with minimal modifications were suitable to be offered as an online intervention. This study explored the perceptions of smokers by using an adapted standalone version of the decision aid (DA), which could be accessed online without the assistance of PNs. To explore whether the DA was also suitable for use without the guidance of PNs, the first aim of this study was to assess the overall usability of the standalone version of the DA. To assess the factors that could possibly be associated with smokers' views on usability, the second aim was to compare groups who differed in their usability score by focusing on their evaluations of the program and their levels of decisional conflict in making a choice. As the main aim of the DA was to increase the use of EBSCIs, the third aim of this study was to explore a possible change in the intention to use EBSCls during a potential cessation attempt. This was achieved by measuring intention to use EBSCIs before and directly after reviewing the DA.

\section{METHOD}

\subsection{Study design and procedure}

A cross-sectional study was carried out in September 2020 via an online research recruitment agency (www.panelclix.nl). The recruitment agency provided a database with 
potential participants who could decide for themselves whether they would take part in the study. After accepting the study invite, participants were automatically transferred to the online questionnaire. At the start of the questionnaire, participants received a brief explanation of the aim of the intervention, followed by the first part of the questionnaire. After filling in the first part of the questionnaire, the participants were asked to review the DA materials, followed by the second part of the questionnaire (all questions are described below). If they completed the entire questionnaire, they received compensation from the recruitment agency. Inclusion criteria were as follows: 1) participants were above the age of $18 ; 2$ ) participants had smoked (primarily cigarettes) in the past seven days and 3) participants were able to understand Dutch and had the necessary internet literacy skills to use the DA. Intention to stop smoking was not an inclusion criteria, but participants had to be willing to consider smoking cessation options. Informed consent was provided prior to the start of the questionnaire by asking if participants wanted to take part in the study and whether they gave the research team permission to use their data for scientific research.

\subsection{Materials}

The DA was named "StopWijzer," which can be translated as either "stop-guide" or "stopsmarter," and it was based on a needs assessment consisting of a literature review (e.g., $(31,69,105)$, individual semi-structured interviews among general practitioners (GPs) ( $n=$ $5)$, practice nurses (PNs) $(n=20)$ and smokers $(n=9)$, a Delphi study on referral to EBSCls $(52,225)$ and the input of an advisory board consisting of experts representing various Dutch smoking cessation related organizations, six of which were actively involved. After the intervention was pilot-tested, the DA was originally deployed to be used in primary care $(228,253)$.

In keeping with the DGSCC $(52,53)$, EBSCls included in the DA were 1) face-to-face counseling (44); 2) counseling via internet (eHealth) $(69,178) ; 3)$ telephone counseling (179); 4) group counseling (74); 5) pharmacotherapy and 6) nicotine replacement therapy. Participants were strongly recommended to use pharmacotherapy and nicotine replacement therapy only in combination with a form of behavioral counseling, as also described in the DGSCC $(52,53)$.

Using non-evidence-based methods, such as acupuncture and e-cigarettes, was also discussed in the DA to address potential questions by smokers about their effectiveness, risks, costs, and availability. The DA discouraged use of these non-evidence-based methods and promoted using EBSCIs as suitable alternatives.

\subsubsection{DA components}

The online DA consisted of the following elements (see also (228)

1. an introduction, which explained the goals and relevance of the DA and summarized the EBSCls and the other elements of the DA: (see Figure 1). 
2. an overview of the different EBSCIs, in the following order: face-to-face counseling; eHealth; counseling via telephone; group counseling; nicotine replacement therapy; pharmacotherapy and the non-evidence-based "cessation" methods of acupuncture (85), laser therapy (188), auriculotherapy (189), hypnosis (190) and e-cigarettes (49);

3. an overview of possible reimbursements of EBSCls by health insurers, with a calculation tool to help patients provide insight into how much money they could save by quitting smoking.

4. The website also contained an overview of the options, which could also be download. The overview listed the EBSCls mentioned above and gave an outline of their target groups, strengths and weaknesses, effectiveness, and costs (see Figure 2).

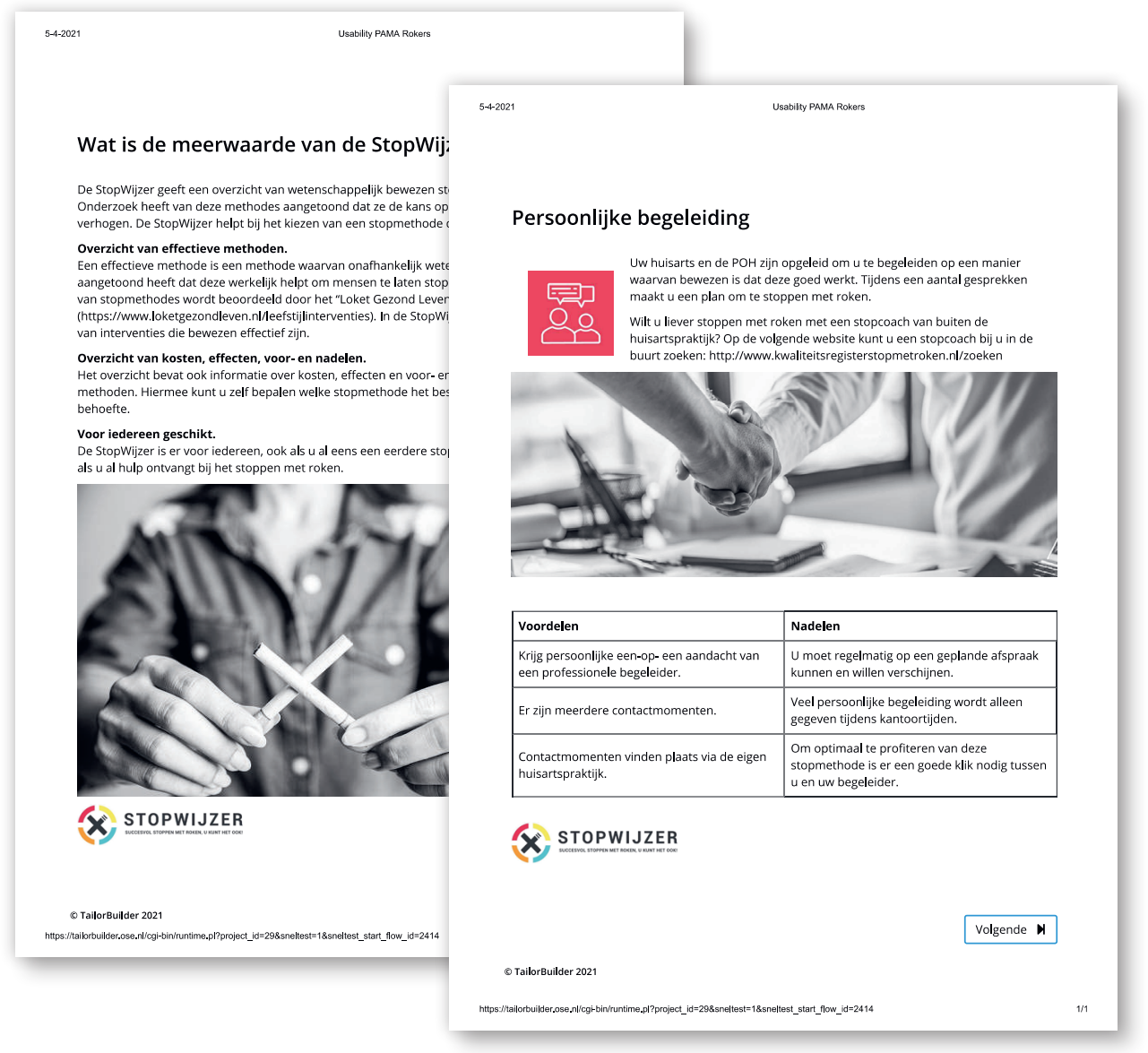

Figure 1. Excerpts from the DA website 


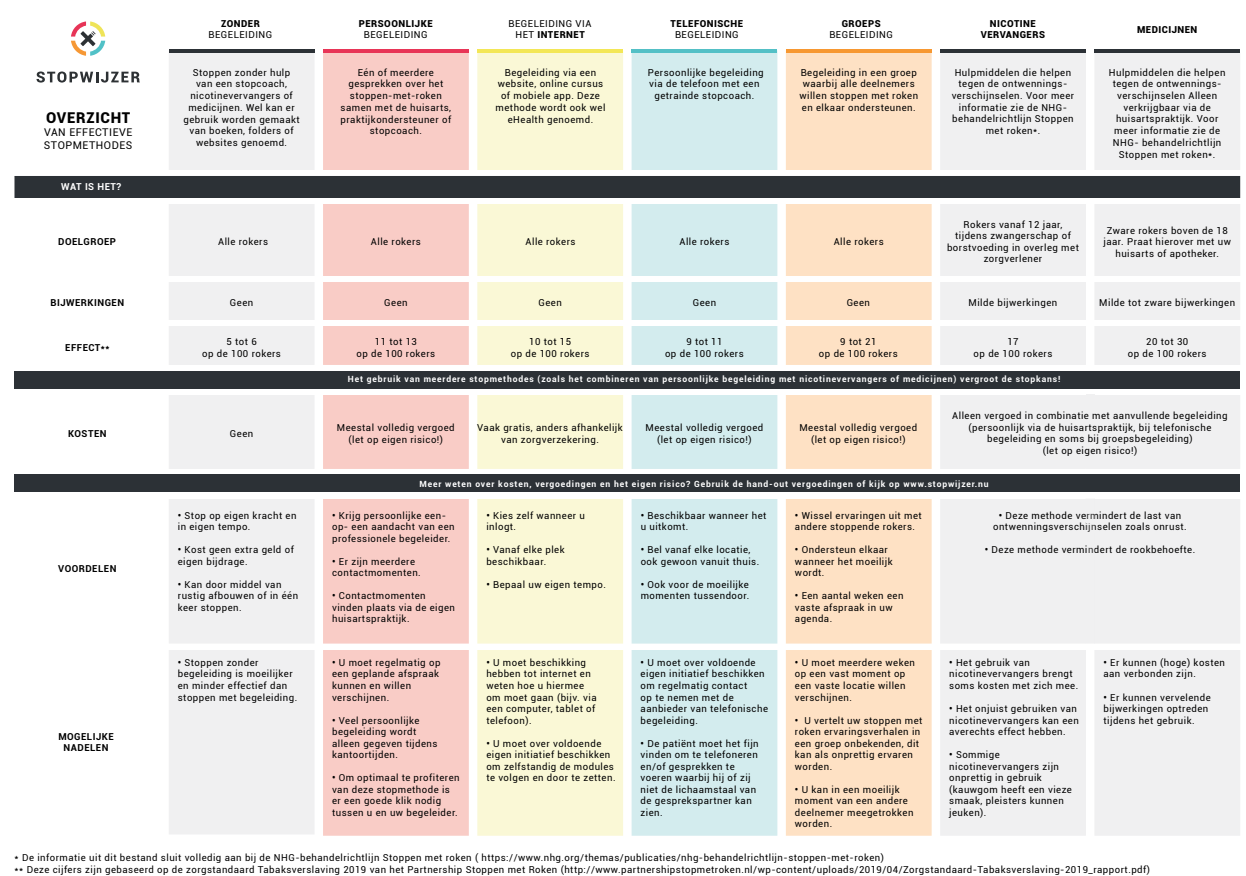

Figure 2. Decision overview (option grid)

\subsection{Measurements}

In terms of demographic variables, we asked the participants about their gender $(0=$ man, 1 = woman), age and highest completed education level ( $1=$ low; $3=$ high).

Smoking behavior was measured with two items asking, "How many regular cigarettes and/or rolling tobacco do you smoke on average per day?" and, "Have you used an e-cigarette in the past 7 days?" ( 1 = no; 2 = yes, with nicotine; 3 =yes, without nicotine; 4 = yes, but I don't know with or without nicotine).

Smoking addiction was measured by using the six items on the Fagerström test for nicotine dependence (FTND), such as, "Do you smoke more often in the first hours after getting up or do you smoke more often during the other hours of the day?". The answers were converted to an overall sum score in which $0=$ not addicted and $10=$ highly addicted (209). Previous quit attempts were measured by asking whether the participant had tried to quit smoking in the past year.

Intention to quit was measured on a five-point Likert scale with one item asking participants if they had the intention to quit ( $1=$ no, definitely not; $5=$ yes, definitely).

Readiness to quit smoking was measured on a six-point scale with one item asking participants whether they intended to quit smoking within a certain period of time $(6=$ yes, within the next month; 5 = yes, within 1-3 months; 4 = yes, within 4- 6 months; $3=$ yes, within 1 year; 2 = yes, within $1-5$ years; 1 = yes, but not within the next 5 years $)(329,330)$. 


\subsubsection{Usability, program evaluation and decisional conflict}

Two items were used to verify whether participants 1) looked at and 2) read the DA materials ( 1 = all the materials; 5 = none of the materials).

The usability of the DA was measured by using the system usability scale (SUS) (331), consisting of the sum of 10 items (e.g., "I found the DA complex"), which could be rated on a five-point Likert scale ( 1 = strongly disagree; 5 = strongly agree), forming a sum score from $0=$ bad usability to $100=$ good usability (Cronbach's $a=.66$ ).

The program evaluation was measured by seven constructs of program evaluation, as also used in previous research (30). Each construct originally consisted of three items measured on a five-point scale ( $1=$ totally disagree; $5=$ totally agree). Negatively-worded items were reverse coded. Table 1 summarizes the concepts measured, example questions and their internal consistency. Based on an unsatisfactory Cronbach's alpha score, one item was deleted from the comprehension subscale. In addition, the adaptation and doseinflicted subscales were omitted from the final scale.

The program evaluation was supplemented with one item enquiring whether participants would recommend the DA to other people willing to stop smoking ( $1=$ totally disagree; 5 = totally agree) and one item asking the participant to rate the overall DA on a scale from one to 10 .

Decisional conflict was measured with the decisional conflict scale (DCS) $(238,332)$, consisting of 16 items (e.g. "I feel I have made an informed choice") on a five-point Likert scale ( $1=$ strongly disagree; $5=$ strongly agree). Table 1 summarizes the concepts measured, example questions and their internal consistency.

\subsubsection{Intention to use EBSCls}

The main goal of the DA was to promote the use of EBSCIs in order to potentially increase their use among smokers when undertaking a quit attempt. Therefore, at the start of the questionnaire and directly after reviewing the DA, participants were asked if they intended to use an $\mathrm{EBSCl}$ if they decided to quit smoking. Participants were presented with 10 options (face-to-face via GP; face-to-face via PN; face-to-face via stop coach; eHealth; in groups; via telephone; NRT; pharmacotherapy; non-evidence-based methods and none), to which their response was measured on a dichotomous scale $(0=$ no; $1=$ yes $)$.

\subsection{Data analysis}

Descriptive statistics were used to describe the characteristics of the recruited participants. Participants were divided into two groups based on their scoring of the usability of the DA using the SUS. As a SUS score above 68 is considered above average for web-based interventions (333), this score was used as a cutoff mark between groups: moderate usability (MU) (mean SUS between 51 and 68) and good usability (GU) (mean SUS above 68). $T$-tests and Chi-square tests were conducted to test the differences between both 
Table 1. Constructs of the program evaluation scale and decisional conflict scale

\begin{tabular}{|c|c|c|}
\hline & Example questions & Cronbach's a \\
\hline \multicolumn{3}{|c|}{ Program evaluation scale constructs } \\
\hline Attention & 'The DA held my attention' & .81 \\
\hline Comprehension & 'In my opinion the DA was clear' & $.81^{1}$ \\
\hline Adaptation & 'The DA applied to me personally' & $.44^{2}$ \\
\hline Appreciation & 'The DA was interesting' & .81 \\
\hline Processing & 'The DA contains good tips on the best way to quit smoking' & .87 \\
\hline Dose infliction & $\begin{array}{l}\text { 'The DA provides a nice overview of the available evidence-based } \\
\text { smoking cessation methods' }\end{array}$ & $.46^{2}$ \\
\hline Persuasion & 'The DA was convincing' & .80 \\
\hline Complete scale & - & .93 \\
\hline \multicolumn{3}{|c|}{ Decisional conflict scale constructs } \\
\hline & By using the $\mathrm{DA}, \ldots$. & \\
\hline Uncertainty & I know what the best choice is for me & .84 \\
\hline Informed & I know which options are available to me & .85 \\
\hline Value clarity & I am clear about which benefits matter most to me & .84 \\
\hline Support & I have enough support to make a choice & .75 \\
\hline Effective decision & I am satisfied with my choice & .78 \\
\hline Complete scale & - & .94 \\
\hline
\end{tabular}

${ }^{1}$ With one item deleted from scale

${ }^{2}$ Subscale was omitted in total program evaluation scale

groups on program evaluation and decisional conflict after reviewing the DA's materials. For the intention to use EBSCls, changes were examined before reviewing the materials (pre-test) and after reviewing the materials (post-test), both in the MU and in the GU group. A paired-sample $t$-test was used to test the pre- and post- difference in the total number of EBSCls that participants intended to use. The McNemar test was used to assess the intention to use individual forms of EBSCls (yes/no), pre- and post- reviewing the materials. To assess whether the intention to use EBSCls after reviewing the materials differed significantly between the MU and GU groups, $\Delta$-scores were constructed indicating the differences pre- and post-reviewing the materials. These scores were compared by means of a $t$-test (total number of EBSCIs) and $\chi^{2}$ analysis (individual EBSCIs).

\section{RESULTS}

\subsection{Study sample characteristics}

The recruitment resulted in 497 participants, most of whom evaluated the DA as moderately usable (MU; $n=393 ; 79.1 \%$ ). Participants were on average 41 years of age, slightly more 
often male than female, had mostly a medium-to-high level of education, had a low-tomoderate level of nicotine addiction, smoked an average of 12.5 cigarettes per day, and generally did not use e-cigarettes (Table 2). Although both groups indicated readiness to quit, in the group of GU smokers this intention was significantly higher. However, smokers from the GU group were not significantly more ready to quit, as both groups indicated being ready to quit within six to 12 months on average.

Table 2. Characteristics of the sample including smoking behavior

\begin{tabular}{|c|c|c|c|c|c|c|}
\hline Study sample characteristics & $\begin{array}{l}\text { Total } \\
(\mathrm{N}=497)\end{array}$ & $\begin{array}{l}\text { MU } \\
(n=393)\end{array}$ & $\begin{array}{l}\text { GU } \\
(n=103)\end{array}$ & $\mathbf{T}$ & $\mathbf{X}^{2}$ & $\begin{array}{l}P \\
\text { value }\end{array}$ \\
\hline Age (years), mean (SD) & $\begin{array}{l}41.23 \\
(13.9)\end{array}$ & $\begin{array}{l}41.06 \\
(13.9)\end{array}$ & $\begin{array}{l}41.96 \\
(12.6)\end{array}$ & -0.597 & & .551 \\
\hline Female, n (\%) & $225(45.3)$ & $172(43.8)$ & $53(51.5)$ & & 1.947 & .163 \\
\hline Educational level, n (\%) & & & & & 0.174 & .916 \\
\hline Low & 59 (11.9) & $47(12)$ & $11(10.7)$ & & & \\
\hline Medium & $229(46.1)$ & $180(45.8)$ & $49(47.6)$ & & & \\
\hline High & $209(42.1)$ & $166(42.2)$ & $43(41.7)$ & & & \\
\hline FTND score ${ }^{1}$, mean (SD) & $4.24(2.4)$ & $4.32(2.5)$ & $3.94(2.4)$ & 1.412 & & .159 \\
\hline Number of cigarettes smoked/day, mean (SD) & $12.51(7.7)$ & $12.56(7.8)$ & $12.38(7.1)$ & 0.215 & & .830 \\
\hline Use of e-cigarettes, n (\%) & & & & & 4.421 & .219 \\
\hline No & $306(61.6)$ & $246(62.6)$ & $59(57.3)$ & & & \\
\hline Yes, without nicotine & $40(8.0)$ & $35(8.9)$ & $5(4.9)$ & & & \\
\hline Yes, with nicotine & $144(29.0)$ & $107(27.2)$ & $37(35.9)$ & & & \\
\hline Yes, do not know with or without nicotine & $7(1.4)$ & $5(1.3)$ & $2(1.9)$ & & & \\
\hline Previous quit attempt undertaken, n (\%) & $309(62.2)$ & $248(63.1)$ & $61(59.2)$ & & .414 & .520 \\
\hline Intention to quit ${ }^{2}$ & $3.97(0.9)$ & $3.88(0.9)$ & $4.29(0.8)$ & -4.334 & & .000 \\
\hline Readiness to quit ${ }^{3}$ & $3.19(1.3)$ & $3.20(1.9)$ & $3.12(1.4)$ & 0.579 & & .563 \\
\hline
\end{tabular}

${ }^{1}$ Range $1-10,0=$ not addicted; $10=$ highly addicted

$21=$ no, definitely not; 5 = yes, definitely

${ }^{3} 1=$ yes, not within the next 5 years; $6=$ yes, within the next month

\subsection{Program evaluation \& decisional conflict}

Both groups mostly appreciated the DA's being comprehensive but expressed least appreciation for the extensive amount of information that the DA contained. Participants from the GU group scored significantly higher on all factors of the program evaluation scale $(P<.001)$, indicating that they found the DA more attractive, understandable, suited to their own needs, useful, valuable in making their decision and persuasive, in comparison to the MU group (Table 3). They also found the level of information provided by the DA of better dosed than the MU group. Participants from the GU group also indicated significantly more 
Table 3. Comparison of mean scores on usability, program evaluation, recommendation to others and grading mark of MU and GU smokers.

\begin{tabular}{llllll}
\hline & $\begin{array}{l}\text { Total } \\
(\mathbf{N}=\mathbf{4 9 7})\end{array}$ & $\begin{array}{l}\text { MU } \\
(\mathbf{n = 3 9 3})\end{array}$ & $\begin{array}{l}\text { GU } \\
(\mathbf{n = 1 0 3 )}\end{array}$ & T & P value \\
\hline Program evaluation scale ${ }^{2}$ & $2.42(0.4)$ & $3.47(0.6)$ & $4.27(0.5)$ & -12.674 & .000 \\
$\quad$ Attention subscale & $3.47(0.8)$ & $3.30(0.8)$ & $4.12(0.7)$ & -9.835 & .000 \\
Comprehension subscale & $3.94(0.7)$ & $3.77(0.7)$ & $4.59(0.6)$ & -11.301 & .000 \\
Comprehension: difficult & $3.79(1.0)$ & $3.60(0.9)$ & $4.53(0.8)$ & -9.191 & .000 \\
Adaptation: fitted situation & $3.45(0.9)$ & $3.32(0.9)$ & $3.97(0.8)$ & -6.581 & .000 \\
Adaptation: lacked information & $3.19(1.0)$ & $3.05(0.9)$ & $3.72(1.0)$ & -6.605 & .000 \\
Adaptation: to general & $3.50(1.0)$ & $3.36(0.9)$ & $4.05(1.0)$ & -6.435 & .000 \\
Appreciation subscale & $3.59(0.8)$ & $3.43(0.8)$ & $4.20(0.6)$ & -9.386 & .000 \\
process subscale & $3.53(0.8)$ & $3.37(0.7)$ & $4.16(0.6)$ & -9.741 & .000 \\
Dose subscale & $3.76(0.8)$ & $3.59(0.8)$ & $4.46(0.5)$ & -11.126 & .000 \\
Dose: much information & $3.77(0.8)$ & $2.69(1.0)$ & $3.57(1.2)$ & -7.518 & .000 \\
Persuasion subscale & $3.74(0.7)$ & $3.57(0.7)$ & $4.38(0.5)$ & -9.051 & .000 \\
Recommendation ${ }^{3}$ & $3.75(0.9)$ & $3.55(0.8)$ & $4.52(0.6)$ & -10.606 & .000 \\
Mark [1 - 10] & & $7.27(1.3)$ & $8.56(0.9)$ & -11.531 & .000 \\
\hline
\end{tabular}

${ }^{1} 0=$ low system usability, $100=$ high system usability

21 = totally disagree, $5=$ totally agree

${ }^{3} 1=$ would not recommend, $5=$ would recommend

often that they would recommend the DA to others who were willing to undertake a smoking cessation attempt and gave the DA a significantly higher mark on a scale from one to 10 , namely an 8.6 (good to very good).

Participants from the GU group reported significantly less decisional conflict, both overall and for the subscales, in comparison with participants from the MU group (Table 4). Both groups reported feeling the most conflicted by a feeling of uncertainty (e.g., "I feel sure about what to choose"). For the MU group, their score on this scale exceeded the cutoff point of 37.5 , which is associated with decision delay or feeling unsure about implementation (334). Smokers from the GU group reported being the least conflicted on their level of being informed, but all their scores fell below the cutoff point of 25 (334), indicating that they perceived themselves as having an adequate overview of the options available to them after reviewing the DA materials (60). The MU group of smokers experienced the least conflict about their level of effective decision making (e.g., "I feel like I have made an informed choice"), although their score did not meet the cutoff point of less than 25 , indicating no substantial certainty to their level of decision making. 
Table 4. Comparison of mean scores on decisional conflict of MU and GU smokers

\begin{tabular}{llllll}
\hline & $\begin{array}{l}\text { Total } \\
(\mathbf{n = 4 9 7 )}\end{array}$ & $\begin{array}{l}\text { MU } \\
(\mathbf{n = 3 9 3 )}\end{array}$ & $\begin{array}{l}\text { GU } \\
(\mathbf{n = 1 0 3})\end{array}$ & T & P value \\
\hline Decisional conflict scale, Mean (SD) & $31.73(14.8)$ & $35.56(13.3)$ & $17.13(10.6)$ & 13.060 & .000 \\
$\quad$ Uncertainty subscale & $34.17(18.0)$ & $38.13(16.7)$ & $19.09(14.2)$ & 10.583 & .000 \\
$\quad$ Informed subscale & $29.60(17.9)$ & $34.01(16.2)$ & $12.78(13.5)$ & 12.224 & .000 \\
Value clarity subscale & $32.88(17.9)$ & $36.70(16.4)$ & $18.28(15.7)$ & 10.218 & .000 \\
Support subscale & $32.34(17.6)$ & $36.28(16.7)$ & $17.31(12.3)$ & 10.792 & .000 \\
Effective decision subscale & $30.18(15.0)$ & $33.40(13.9)$ & $17.90(12.7)$ & 10.255 & .000 \\
\hline
\end{tabular}

$15=$ no decisional conflict, $100=$ a lot of decisional conflict

\subsection{Intention to use EBSCls}

The third aim of this study was to explore a possible change in the intention to use EBSCls during a potential cessation attempt. Participants in both groups reported an overall and significant higher intention to use more EBSCls after they had reviewed the DA materials, in comparison to their intention before reviewing the materials. Regarding individual forms of EBSCls, this difference was specifically significant for their intention to use eHealth. The intention of the participants to not use any EBSCI when making a quit attempt significantly decreased. No differences were found regarding the usage of non-EBSCls (NEBSCIs).

Furthermore, participants from the GU group had showed a significantly higher increase in intention to use more EBSCls eHealth after reviewing the materials in comparison with the MU group. 
Tabel 5. Comparison of the intention to use EBSCls measured before and after reviewing the DA

\begin{tabular}{|c|c|c|c|c|c|}
\hline & \multicolumn{2}{|c|}{ MU (n=393) } & \multicolumn{2}{|l|}{ GU $(n=103)$} & \multirow{2}{*}{$\begin{array}{l}\text { Comparison of } \\
\text { changes } \\
\text { between MU } \\
\text { and GU }\end{array}$} \\
\hline & Pre & Post & Pre & Post & \\
\hline $\begin{array}{l}\text { Intention amount of EBSCI } \\
\text { to use, mean (SD) }{ }^{1}\end{array}$ & $1.47(1.1)$ & $1.59(1.1)^{\star \star}$ & $1.89(1.4)$ & $1.91(1.1)$ & NS \\
\hline \multicolumn{6}{|l|}{ Behavioural counseling, \% (n) } \\
\hline via GP & $10.2(40)$ & 9.9 (39) & $17.5(18)$ & $17.5(18)$ & NS \\
\hline via PN & $15.8(62)$ & $18.3(72)$ & $24.3(25)$ & $23.3(24)$ & NS \\
\hline via stop coach & $12.7(50)$ & $16.0(63)$ & $15.5(16)$ & $15.5(16)$ & NS \\
\hline eHealth & $9.9(39)$ & $16.3(64)^{\star \star \star}$ & $12.6(13)$ & $30.1(31)^{\star \star \star}$ & $\Delta \mathrm{GU}>\Delta \mathrm{MU} * \star$ \\
\hline in groups & $7.1(28)$ & $8.1(32)$ & $1.9(2)$ & $4.9(5)$ & NS \\
\hline via telephone & $7.6(30)$ & 9.9 (39) & $6.8(7)$ & $10.7(11)$ & NS \\
\hline NRT & $25.2(99)$ & $24.9(98)$ & $47.6(49)$ & $40.8(42)$ & NS \\
\hline Pharmacotherapy & $13.0(51)$ & $15.8(62)$ & $25.2(26)$ & $23.3(24)$ & NS \\
\hline $\mathrm{NEBSCl}^{2}$ & $8.7(34)$ & $7.9(31)$ & $11.7(12)$ & $8.7(9)$ & NS \\
\hline None & $37.2(146)$ & $31.8(125)^{\star \star \star}$ & $26.2(27)$ & $16.5(17)^{\star \star}$ & NS \\
\hline
\end{tabular}

${ }^{1}$ other category excluded

${ }^{2}$ e.g., acupuncture, hypnotherapy, or laser therapy

* $P<0.05, * \star P<0.01, * \star * P<0.001$

\section{DISCUSSION}

The aims of this study were to 1 ) investigate the overall usability; 2) compare groups who rated the DA with, respectively, a moderate and good usability on their evaluation of the program and 3 ) to explore a potential change in the intention to use EBSCls before and directly after reviewing the DA.

With regard to the first objective, the results suggest that most participants found the DA moderately usable in the form in which it was presented, whereas smokers willing to quit scored the DA's usability as good. Although both groups had an intention to quit smoking, this intention was significantly higher in the participants from the GU group. A higher intention to quit might also indicate greater interest in the materials, given that according to socio-cognitive models such as the health belief model (335), the theory of planned behavior (112) and the I-change model (105), a person's beliefs about the effectiveness and perceived benefits-among other factors, such as perceived susceptibility, severity, and barriers-might regulate a person's interest in a behavior change. Furthermore, research has shown that smokers contemplating quitting within the next six months, but not within the coming month (336), might benefit the most from information about the intended behavior and from self-efficacy-enhancing information (337). Therefore, smokers from the GU group may have regarded the information as more 
relevant for them, which might have resulted in more information retention and absorption and a higher usability score.

The second aim of this study was to compare groups who scored the DA with moderate and good usability on their evaluation of the program (measured by a program evaluation scale, willingness to recommend the DA to others and scoring the program with an overall mark ranging from one to 10) and their level of decisional conflict. Both groups differed on all aspects, which gives indication for a possible relationship between usability, program evaluation and DCS; these factors also displayed a significant but medium correlation in relation to each other. As the DCS measures the perceived conflict in the decision-making process, more conflicted feelings might also regulate the level of usability and appreciation of the DA. Further research is needed to explore the possible relationship between these three concepts in order to provide more in-depth insight into these connections. Both groups found the DA to be comprehensive, although they also indicated that the materials contained an extensive amount of information. Extensive information can be effective for higher educated users, such as those in our sample, as they may benefit from the processing of more in-depth information (338). However, to also reach less-educated groups of users, it is important in stimulating comprehension and attracting attention that this information be made accessible, and these aspects of the DA were less well-rated in this study. Overall, the DA was positively received, with both groups giving it a satisfactory grade.

Regarding decisional conflict, both groups expressed a high level of uncertainty about how to make the actual decision for an EBSCI (e.g., "I feel sure about what is the EBSCI for me"), even though they also reported that they had an adequate overview of the available EBSCls. This might indicate that even though the participants felt informed about the EBSCIs, they were not sure how to make a balanced decision that aligned with their own preferences. As DAs are designed to aid in the informed decision-making process, they should not only provide all relevant information on the available options but also include values clarification exercises or methods (e.g., exercises aimed at helping users evaluate a wide range of options in their own specific contexts) to determine which of the options is most fitting to their needs $(127,129,130)$. Another explanation for this might be that not all smokers had the intention to quit at the time of reviewing the materials and did not yet, therefore, think deeply about this part of the decision-making process.

The third aim of this study was to explore a possible change in the intention to use EBSCls during a potential cessation attempt by measuring intention before and directly after reviewing the DA. A slight but significant increase was found in the total number of EBSCls that participants intended to use. The number of participants willing to use eHealth after reviewing the DA materials also increased. Although systematic research about the (cost-)effectiveness of existing eHealth interventions is still scarce $(70,71)$, available studies that report on its effectiveness are positive $(67-69,178,339-341)$. The demand for eHealth interventions, as found in this study, necessitates a greater supply of validated (i.e., 
evidence-based, and effective) eHealth interventions. Furthermore, since there are also numerous internet interventions available that are not evidence-based (70), the potential establishment of a certification by which smokers could recognize validated eHealth interventions might further increase the willingness to use eHealth, as this would help the smoker in the decision-making process.

The results also indicated a significant decrease in the number of participants who stated that they would make a cessation attempt without the help of EBSCls. This finding is consistent with the aim of the DA, as EBSCls are proven to double the likelihood of successful smoking cessation (86). A significant decrease in the intention to use other non-evidence-based smoking cessation interventions such as acupuncture and laser therapy was not found (85). As research has shown that smokers use NEBSCls almost as often as they use EBSCls (91), more attention should be paid to understanding why ineffective methods are still preferred by some smokers and which information they may need to steer them away from these options.

\subsection{Potential strengths and limitations of the study}

One of the strengths of this research was the use of validated questionnaires to measure the relevant constructs. Another strength was the inclusion of a large proportion of smokers who were willing but not yet ready to quit (those in the contemplation phase), in contrast to other studies that usually include self-selected smokers who were ready to make a quit attempt. This factor yielded the added advantage that smokers were likely not to have sought information on EBSCls prior to the study or had decided on a form of EBSCI beforehand. However, this also included a limitation, as smokers with no intention to quit might look for other information during that phase. However, all smokers were informed of the aim of the study in advance and were instructed to take on the mindset of someone who was willing to quit smoking within a short period before and after reviewing the materials and during the questionnaire.

The second limitation was that the DA was primarily developed to be used with the aid of a PCP, such as a PN, in the PCS $(228,253)$. The content of the DA, however, was developed using a theoretical grounding based on relevant constructs from previous studies $(31,52$, $69,105)$, a needs assessment in the form of a Delphi study (225) and the input of an advisory board. The DA used in this study was adapted by rewriting the materials to fit within the smokers' frame of reference, taking into account patients' potentially low health literacy and rewriting the information using clear and comprehensible language, in accordance with the applicable Dutch guidelines (Language level B1) (186).

The third limitation was using a cross-sectional design (342) instead of a more longitudinal design, such as an randomized controlled trial, as was used in previous research on the DA materials (253). Therefore, conclusions on the effectiveness of the intervention in a real-life setting could not be drawn. However, as the main aim of this study was to 


\section{CHAPTER 7}

explore the usability of the materials, this study serves as a pilot test for potential further development of the DA materials.

The last limitation was the use of an online research agency, which resulted in the recruitment of a relatively highly educated participant sample. An additional consequence might be that participants only took part for the compensation they would receive from this agency and, therefore, did not complete the questionnaire carefully. This was guarded against by including a warning that participants who did not fill out the questionnaire would not receive a reward. The researchers also screened the data for time of completion and to exclude participants who fell below the average completion time, but this did not result in the exclusion of any participant.

\subsection{Practical implications}

As almost $80 \%$ of the group of participants rated the materials of the DA only moderately usable, the researchers can cautiously conclude that the materials in their current form are not usable as a standalone DA. To adapt the DA in a way that best fits its potential users, qualitative studies such as read-aloud interviews or pilot groups could aid in pinpointing concrete facilitators and barriers for the usage and reception of the DA. To draw conclusions on the effectiveness of the DA on EBSCl usage and effectiveness, randomized controlled trials conducted in ways described in similar research (343) are recommended. In order to decrease the amount of information within the DA, information provided to users could be tailored to their prior knowledge or levels of interest $(341,344)$. A further communication strategy to also reach a greater number of less-educated smokers might be including more video-based information, as previous studies suggested the advantages of using videobased over text-based communication $(69,345,346)$. Last, as participants in this study indicated that they found it difficult to make a firm decision, the use of values clarification methods could aid in steering the decision-making process by helping users explore their preferences $(127,129,266)$.

Furthermore, based on the findings of this study and their own experiences with the DA in the primary care setting (253), the researchers suggest that the utilization of a hybrid version (i.e., blended care) that could be used both within the PCS and as a standalone option could be a feasible option for further development of the DA. As mentioned above, PCPs in the PCS work with the DGSCC $(52,53)$, which are based on the $5 \mathrm{~A}$ protocol (i.e., ask, advise, assess, assist and arrange) (177). However, as time within the PCS is very limited, an abbreviated version of this protocol has been proposed, the ask-advise-refer (AAR) strategy (242), which can be used to structure very brief advice by a PCP and has already been proven in Dutch cardiac wards (117). PCPs can use the DA as a reference during the referral part of this strategy, while smokers can use the online materials to further explore the available EBSCI options after their consultation with the PCP. Another advantage of adapting the DA into a hybrid variant is that it may benefit from internet interventions' 
broad reach but could also have the advantage of the higher adherence rate of interventions used in healthcare settings (132). Another advantage of so-called blended care is that it allows the combination of personal attention and synchronous communication with the online advantages of high accessibility $(50,347)$. Given that the primary care setting prominently reaches smokers who are more motivated to quit $(132,348)$, using a mass media approach might reach a greater absolute number of smokers, even those who are still in a (pre-)contemplating phase (132), as was also the case in this study.

\section{CONCLUSION}

As the use of EBSCls can double the likelihood of a successful smoking cessation attempt, this study investigated the usability of a DA aimed at increasing the use of EBSCls. As the DA was originally designed to be used in general practice with the guidance of a PN, the aim of this study was to explore the usability of an adapted standalone version of the aid among a large group of smokers. Most participants found the DA only moderately usable, although those who intended to quit found it more usable. Participants who found the usability of the RA to be good rated higher on all elements concerning the evaluation of the DA, including the recommendation to others and overall mark, and experienced less decisional conflict. Furthermore, after reviewing the DA, participants on average had a significantly higher intention to use more EBSCls, in particular in the form of eHealth. Recommendations to make the DA more usable and well-received among a broader group of smokers could include tailoring, transforming text-based information into video-based information and including values clarification methods. Furthermore, as the DA was found to be only moderately usable in the standalone version, a hybrid variant that would allow smokers to use the DA both on their own and with the guidance of PCPs could aid both groups in choosing a fitting EBSCl option. 


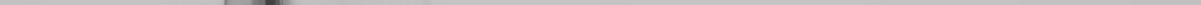




\section{CHAPTER 8}

General discussion 


\section{CHAPTER 8}

The overall aim of this dissertation was to explore the potential of a referral aid (RA) regarding evidence-based smoking cessation interventions (EBSCls) in the primary care setting (PCS). The goal of the RA was to increase the number of referrals to EBSCls by educating primary care professionals (PCPs), particularly practice nurses (PNs), about the effectiveness of these interventions and by facilitating the referral process. In Section 1 of this chapter, the main findings of all studies described in this dissertation are summarized and discussed. This section is divided into Part I, which explores the potential of RA in the PCS, and Part II, which explores further applications of the RA partially outside of the PCS. We then turn to methodological and practical considerations of the studies described in Section 2, followed by implications and recommendations for future research in Section 3. Finally, general conclusions are presented in Section 4.

\section{SUMMARY OF THE MAIN FINDINGS}

\section{Part I: The potential of an RA for the PCS}

The first part of the dissertation examined the potential of an RA intended to increase the use of smoking cessation interventions for smoking patients within the PCS.

\subsection{Health-care professionals benefitted from an overview of available EBSCIs in their doctor-patient communication}

Chapter 2 described a systematic exploration and consensus (Delphi) study, which focused on obtaining an overview of the knowledge, experiences, and viewpoints of smoking cessation experts (researchers and PCPs) on the effectiveness and use of EBSCls. Although a wide range of EBSCIs with a strong evidence base are already available to refer to in daily practice (142), referral by PCPs to these EBSCls is often limited and interventions often remain underused (12). The use of evidence-based interventions to support smoking cessation can significantly increase the success rate of quit attempts (86). We conducted this study to identify the existing knowledge and perceptions of PCPs and smoking cessation researchers regarding the effectiveness of EBSCls as well as to explore possible gaps in that knowledge. Both groups were in high agreement on what patient characteristics should be considered when choosing an appropriate EBSCIs, the most crucial of which were considering the patient's needs and previous cessation attempts; furthermore, both groups scored highly on agreement concerning the use of special protocols for high-risk groups of patients. However, these groups did not reach a consensus on the effectiveness and value of e-cigarettes as a means of quitting. Furthermore, we found a lower degree of consensus regarding the effectiveness of EBSCls among PCPs. We therefore concluded that identifying the needs of PCPs with respect to EBSCls and their use can provide insights into how to promote higher intervention uptake in primary care. Furthermore, information 
on the effectiveness and use of EBSCIs should be made available in a format that is easily referable for PCPs. Based on these findings, we developed an RA for the PCS with information on EBSCls, including their effectiveness, advantages, disadvantages, mode of use, and costs. The aim of the RA was to promote the use of EBSCls among smokers who want to quit smoking and thus to increase smoking cessation success rates.

\subsection{Caregivers were relatively positive about the use of an RA for smoking cessation interventions in primary care, but adherence and usage did not reflect this}

Previous studies have found that PNs' adherence to smoking cessation guidelines (54, 58-60), particularly the step that entails the referral of patients to EBSCls, is suboptimal $(31,58,59)$. Therefore, we developed an RA to aid PNs in adhering to this step. The newly developed RA was named "StopWijzer," which translates to "Stop guide" or "Stop smarter." Chapter 3 described the study design of the development and evaluation of this information and decision tool for supporting PNs in guiding smoking patients and referring them to EBSCIs. The RA was then tested in the PCS through a randomized controlled trial (RCT). The process of recruitment among both PNs and smokers was tracked, as was intervention appreciation, level of informed decision making, and cessation effectiveness. As described in Chapter 4, recruitment of both PNs and smokers resulted in low numbers of participants. In the PN group, only 73 approached PNs were willing to participate, which was a small percentage (4.4\%) of those approached. Furthermore, of those who participated, 20 PNs did not recruit a single patient. Ultimately, 285 smokers were recruited to participate in the study. Of the total 285 participants registered by the PNs, 157 (55\%) filled in the baseline questionnaire. There was also a high dropout between the baseline questionnaire and the 6-month follow-up questionnaire (nearly $48.1 \%$ ), which is quite common in patient trials with minimal (and prominently online) personal contact with the research team (349). Although PNs and smokers were relatively positive about the referral and rated the materials an 8 (smokers) and an 8.6 (PNs), the aid was not used intensively, and no significant effects on the discussion and use of EBSCIs nor on abstinence could be found when comparing these outcomes with the control condition, in which smokers did not receive an RA. Furthermore, we assessed whether the range of EBSCls outlined in the decision aid (DA) might induce or reduce decisional conflicts among patients. The findings revealed that the RA did not result in additional decisional conflict in the experimental group. Although Chapter 3 also proposed a plan for conducting a cost-effectiveness study, this sub-study was not included in Chapter 4 due to the low response rate and a lack of effects in smoking cessation. Since the RA was well-received by both PNs and smokers and a higher number of EBSCls were used in the experimental condition (especially in the form of eHealth, group counseling, and nicotine replacement therapy, where this increase was significant), the RA is potentially suitable for implementation in the PCS. We concluded that further research should determine how to facilitate the adoption of the RA within the PCS as well as how to better involve PNs and smokers in recruitment to an RCT. 


\subsection{Although the RA was positively received by PCPs, intention to adopt was low}

To explore facilitating factors and barriers that influence the potential willingness of PCPs to adopt the RA in its current form in their daily practice, we conducted a cross-sectional study among PCPs. To recruit a sufficient sample of PCPs, and to ensure that all PCPs with the same level of experience with the RA, we had to include PCPs who had not yet worked with the RA in previous studies. Our findings were presented in Chapter 5 , which described the factors underlying the PCPs' intention to adopt the RA determined by assessing the differences between PCPs in charge of smoking counseling in the general practice with or without the intention to adopt. Although appreciation in both groups was high (both groups scored the RA materials higher than an 8), most PCPs did not intend to adopt the RA. Nonadopters had a more negative attitude toward the RA (i.e., they perceived fewer advantages and more disadvantages), experienced less social support, had low self-efficacy, and faced barriers such as a lack of time and skills. Recommendations for facilitating the adoption of the RA in the PCP are as follows: First, the RA itself should be improved through a second round of co-creation focused on the adoptability of the tool in practice; second, the added value of referring patients to EBSCls should be better communicated through implementing the RA in smoking cessation training for PCPs. Making the RA part of this training could also increase PCPs' attitude, social support, self-efficacy, and perceived skills regarding the use of the RA.

\section{Part II: Future applications and possibilities}

Because a lack of time for using the materials in practice was a frequently reported barrier in Chapter 5 as well as in previous research (61), we sought to explore the usefulness of a standalone or hybrid version of the RA. Thus, the RA could be used in the way that the original RA was intended, but also by smokers in a standalone version (with no counseling by a PCP) or as a strategy for preparing smokers for their counseling session with a PCP, which could also help to reduce potential time barriers for PCPs. To explore this further, we examined the use of DAs for making a decision about a health behavior and conducted a usability study with a large group of smokers outside of the PCS, investigating whether the RA materials were usable without guidance from the PCP.

\subsection{Although most DAs included behavior change elements, only some reported behavioral effects}

To explore the potential of interventions designed to aid the decision-making process aimed at behavior change, we conducted a scoping review (Chapter 6). The aim of the scoping review was to broadly synthesize literature regarding DAs for supporting decision making about diet, physical activity, sleeping, and substance use. We found that all included DAs offered information about the behavior; approximately $70 \%$ of the studies reported that they used behavioral explanation and change theories such as self-determination theory 
(350) or the theory of planned behavior (112), value and/or preference clarification models, and many other elements (e.g., goal-setting) to assist users in making a choice. However, effects were mixed and only a few studies used standardized measures, such the decisional conflict scale, to measure outcomes. Some positive behavioral effects were reported, especially on smoking cessation (as demonstrated by previous research (270)). We concluded that DAs are potentially beneficial for supporting people to change health behaviors, including and especially smoking cessation.

\subsection{The RA seemed useful in a standalone variant}

As the number of smoking patients recruited in our main study (described in Chapters 3 and 4) was very low, we were unable to fully investigate the usability of the RA because of our limited sample of participating smokers. To draw meaningful conclusions on the actual usability of the RA materials among smokers, and to simultaneously examine whether the RA had potential to be used as a standalone intervention (taking more of a DA form), we conducted an additional usability study among smokers. By using an online research recruitment agency, we included 497 smokers from the general population. Intention to quit smoking was not an inclusion criterion. The materials for the DA used in this study were adapted by rewriting the materials to fit the smokers' frame of reference (e.g., the information was directed at a smoker rather than a PCP). Smokers from this sample were instructed to review the materials and subsequently evaluate them directly before and after reviewing the materials by means of an online questionnaire. They were asked to give their opinion on the RA in case they made a quit attempt. The aims of this study (as described in Chapter 6) were as follows: (1) to conduct a usability evaluation; (2) to conduct a program evaluation (on whether the program holds the attention, is comprehensive, adapts to user needs, is appreciated, is easily processed, holds a fitting amount of information, and is persuasive) and to evaluate decisional conflict after using the RA; and (3) to determine a possible change in intention to use EBSCls before and directly after reviewing the DA. Most smokers only evaluated the DA as moderately usable $(n=393$, $79.1 \%$ ). Smokers who rated the usability as good scored higher on all elements of the program evaluation and experienced less decisional conflict, but also displayed a higher intention to quit. After reviewing the DA, participants on average had a significantly higher intention to use EBSCls, and specifically indicated being more willing to use eHealth. Recommendations for making the DA more usable and well-received among a broader group of smokers are as follows: First, the DA could provide more targeted communication by tailoring the content specifically to the user's prior knowledge or interests (337). This may decrease the amount of information provided per user and increase relevance and information processing $(341,344)$. Second, text-based information could be transformed into video-based information to make the information more understandable and absorbable, as described in other studies for a similar research sample $(69,345)$. Third, value 


\section{CHAPTER 8}

clarification methods should be included to support smokers in the decision process without the help of PNs $(127,129,266)$. Furthermore, the DA was evaluated as useful by smokers who were ready to quit; this tool may be useful for guiding their smoking cessation process. Yet, smokers who were less ready to quit found the DA only moderately usable and further development is thus relevant. A more in-depth exploration of the needs of this particular target group is required as well as a more thorough co-creation process that involves them. A potential outcome could be a hybrid variant where smokers can use the DA on their own and with the guidance of PNs, which could aid both groups in choosing a fitting EBSCI option. This could represent a feasible future application.

\section{METHODOLOGICAL AND PRACTICAL CONSIDERATIONS}

As evidenced by numerous international and national studies in the field of smoking cessation in primary care, recruitment, and implementation of research in the PCS is a challenging task. During our studies, we encountered several methodological and practical considerations regarding research conducted among PNs in the field of smoking cessation, which are outlined in the following subsections.

\subsection{Facilitating recruitment and adherence of PCPs in research}

PNs were introduced in the PCS more than 25 years ago (41). The aims of this new profession within the PCS were to reduce the workload of GPs and to provide protocol-based patient education, disease management and prevention, and lifestyle counseling for chronic conditions $(42,351,352)$. This led to a shift in responsibilities, where the GP was still the first point of contact for smokers as they often focus on providing brief quitting advice and referring smokers who want to quit to the PN for more intensive counseling. However, a study by the Netherlands Institute for Health Services Research (Nivel) demonstrated that, because of the more intensive care that PNs provide, PNs now also suffer from a high workload $(42,353)$. This problem negatively affected the recruitment of PNs and patients in our research. This was not only the case for the main part of the study (Chapters 3 and 4) but also for the other studies among PNs and PCPs (Chapters 2 and 5) and during other communication attempts with this group (during the developmental process and during the main study).

\subsubsection{Getting into contact with the PCS}

To recruit a sufficient sample of PNs in our studies, we employed multiple methods of approaching them (described in Chapter 3 and 4 ) based on earlier projects in the PCS (28, $31,65,69,252,354)$. However, none of these methods were particularly effective as only 73 out of all 1663 approached PNs (4.4\%) were willing to participate in our study. Initial 
attempts to contact PNs by telephone were sometimes cut off by the practice operator or assistant, and as there was a very low response rate to our emails (less than 1\%), this was the only route for direct contact with the target group. In the other studies where PCPs had to be recruited, response rates varied between $27.7 \%$ (in the largest round of the Delphi study described in Chapter 2) and 34\% (recruitment of new PCPs for the adoption study, as described in Chapter 5), and they were recruited by sending emails to the general practice where the PCP worked. These response rates can be explained by the time and effort required for participation being lower than in our main study. When contact was made with PNs, the reasons they provided for not participating in our main study concerned having other priorities than smoking cessation interventions and research, lacking time, being on special leave, or being otherwise disposed. A few PNs mentioned that they had already participated in other studies or were presently participating in a study concerning smoking cessation and were therefore not eager to participate in our study. This is similar to responses that GPs give when asked to participate in studies, with the addition that GPs also report a gap between the general practice setting and research, which was also described in Chapter 2 of this dissertation (355). Although this is not uncommon for research studies conducted in the PCS $(31,134,233-235)$, it did lead to a lower statistical power and generalizability of the results obtained in this study (236).

\subsubsection{Managing expectations}

One way to fit participation in research into the daily routine of PNs is to manage expectations in advance, such as by informing potential participants extensively about all of the content and timelines of the elements of the study; thus, they would be able to make an educated assessment of whether they are able to participate in the study and what it entails (237). During the recruitment process of this study, potential participating PNs were informed about the actions that they were expected to perform with respect to the RA and the research by means of an information letter. In this letter, we also outlined the timeframe of the study and provided a scenario of what working with the RA might likely look like in practice based on estimates from experts involved in the pilot test. However, to include a more accurate description of the actions, more thorough pilot testing had to be conducted among PNs who actually worked with the materials. Thus, a more accurate estimate and more detailed pinpoints could be provided in the information letter, for example, regarding how to ensure that PNs would not forget the study during their daily routine.

\subsubsection{Nonusage attrition}

Thoroughly informing the PNs of the actions that they are required to perform for the purposes of the study might also help to decrease nonusage attrition, which our study also suffered from. Nonusage attrition refers to participants remaining in the study but not actively participating. In our study, a proportion of the PNs (27.4\%) did not recruit any 


\section{CHAPTER 8}

smokers during the trial and thus did not use the RA (219). When PNs in our main study were asked why their adherence was low (by means of qualitative questions asked in the study, as described in Chapter 5), they named barriers such as lacking time, forgetting to bring up the materials, and lacking patients, which are known reported barriers to program implementation $(356,357)$. As many PNs indicated that their nonusage attrition stemmed from forgetting to use the RA during their counseling sessions, we attempted to use periodic emails and phone calls as reminders of their participation in the study. Although reminders have been proven to be effective at facilitating behavior change, providing too many reminders can be perceived as excessive interference and can reduce their effectiveness as PNs tend to block them (358). To counteract this, we attempted to increase engagement by, for example, inviting PNs to send in tips and tricks for recruitment to our monthly newsletter (359). We aimed to keep nonusage attrition to a minimum by making the recruitment of smoking participants as easy as possible, which we did most crucially by providing sufficiently informative materials to patients to inform them about the study. These materials could be discussed during the consultation or taken home by the patients to return to at a later date.

\subsubsection{The role of reimbursement}

In all of the studies described in this dissertation that dealt with the recruitment of PCPs, we offered reimbursement in the form of gift cards to stimulate participation. In the case of the studies described in Chapters 2 and 5, participants received a gift card of a fixed value after completing the study. In our main study, to prevent attrition and stimulate active recruitment by the PNs, the amount of money that PNs could earn was directly in line with the number of patients they recruited (ranging from $€ 20$ for recruiting at least one participant to $€ 100$ for recruiting at least five participants). However, this approach was not particularly effective, as 20 PNs did not recruit a single patient and PNs who did recruit patients only recruited an average of five smokers each (no differences existed between the experimental and control conditions). Other methods that we used to stimulate PNs to recruit smoking patients are discussed in Section 2.2.

In sum, to facilitate the recruitment of PNs and their adherence during the study, in addition to minimizing the tasks associated with the research, it is important to thoroughly inform them of the time commitment expected of them. Doing so will enable them to accurately assess whether participating is feasible within their daily routine. Financial incentives as rewards for participation did not seem to be effective in our study. In addition, to facilitate the research becoming part of the PNs' daily routine, regular reminders should be sent to them to keep the research fresh in their minds. Further research should be conducted to determine the most appropriate number of reminders and the format in which they should be sent to PNs. 


\subsection{Facilitating recruitment and adherence of smoking participants in research}

As mentioned previously, recruitment of smokers during the RCT was also lower than expected (see Chapter 4). As PNs do not pause their routine to perform actions purely for research (e.g., explaining the rationale behind a questionnaire or filling in the questionnaire during the time that could be used for cessation counseling), to facilitate the recruitment of smokers, researchers should aim to make this process as easy as possible. Although we used minimal exclusion criteria to include a large and varied range of patients, the recruitment rate by PNs remained low. Furthermore, a recent study indicated that equipping PCPs with the knowledge and skills required to refer patients and motivating health care provides to discuss various counseling options with patients can be potentially successful strategies for reaching smokers to provide smoking cessation counseling (360). The main reasons that the PNs gave for the low recruitment were as follows: (1) not seeing any smoking patients during the study; (2) lacking the time to recruit smoking patients; and (3) mostly encountering smokers who are not motivated to quit or to participate in research.

\subsubsection{Encountering smokers}

First, we found the PNs' statements that they did not encounter smoking patients during the recruitment stage of the RCT (a period of 6-12 months, depending on when the PNs entered the study) to be somewhat peculiar, as this would not be expected given the prevalence of smoking in the Netherlands and the frequency with which Dutch smokers visit their GP (37). One explanation may therefore be that the PNs meant that they did not encounter smoking patients to whom they had not already provided brief cessation advice, probably without resulting in an intention to quit, or whom they otherwise would encounter more often during counseling sessions (61). An example of the latter are smokers with COPD who visit the PCS regularly for routine COPD management checkups (361). Because of their regular contact with PNs, smokers with COPD tend to receive more advice on smoking cessation than smokers without COPD. However, research suggests that they do not undertake more attempts to quit, which might demotivate PNs to continually provide them with smoking cessation advice and counseling (361). In addition, a study conducted among smokers with respiratory disease discovered an "advice limit," after which smokers got tired of the repeated messaged and "blocked" the advice out (362). However, as most smokers find it difficult to become motivated to quit without the guidance of a PCP, such as that provided by the RA, this is a missed opportunity $(363,364)$.

Using a more passive form of recruitment might be suitable for smokers who have already received cessation advice at an earlier date but were at the time not yet ready to quit smoking. Placing recruitment and advertising materials in the general practice without the active recruitment efforts of a PCP has been proven effective for recruitment in another study (64). During the recruitment phase of the main study (described in Chapters 3 and 4), we already used such materials in the RCT by providing PCS (digital) posters and flyers, 


\section{CHAPTER 8}

which could be exhibited in the waiting room. As we did not investigate the way in which smokers were notified of the RCT in depth, we are not able to report the success rate of these materials. Furthermore, although this method would not reach smokers who are unwilling to quit smoking, it would allow scientists and PNs to reach a broader group of patients without actively putting in time and energy themselves.

\subsubsection{Addressing the limited time frame}

With regards to the limited time frame PNs could be spending on the recruitment process during their daily routine, we tried to limit the time burden for both PNs and patients as much as possible. We provided questionnaire packages (containing an information letter, the baseline questionnaire, and an envelope with return postage) to all participating PCSs so they could be handed out to smokers who did not have the Internet or a form of technology to fill in the online questionnaires. We also provided the PCSs with business cards so they could redirect smokers with questions concerning the research to the research team. To register smokers for the study, we required only their name, date of birth, and email address and/or phone number from the patient to be submitted by the PCP through our website (submitting the information by email or telephone was also possible but was only used sporadically). Most smokers chose to provide us with their email address, which we used to give them automatic access to the information on the closed section of the website without having to sign up separately. Patients who did not make use of the paperbased questionnaires also received their questionnaires by email, which allowed the research team to send multiple reminders using an automated system. The online questionnaires were designed to be viewed on multiple platforms ( $\mathrm{PC}$, smartphone, or tablet), making it easy for participants to fill them in (365). By requesting only minimal contact information, we hoped to reduce the privacy concerns of potential participating smokers. However, this also had drawbacks as it left us with minimal patient contact information, which made follow-up contact more difficult since we did not have the ability to communicate more personally than by email, which we could only do by name $(349,365$, 366). A potentially high level of perceived anonymity might also have made it easier for patients to drop out of the trial (219).

\subsubsection{Readiness to quit}

Lastly, a large proportion (67\%) of our sample of smokers was ready to quit within 1 month. Only a small percentage (smaller than $1 \%$ ) was only willing to quit within 6-12 months, or even after a longer time period. This is in line with previous research, which suggested that patients who are not yet sufficiently motivated to quit often do not receive the same level of smoking cessation counseling as those who do, and might therefore not have been asked to participate in the study by the PN (61). Although the RA described in this dissertation might be used as a new approach to open the conversation about smoking cessation, we 
suspect that the RA would not be highly effective in groups of smokers who do not have the intention to quit smoking yet. This is because the main focus of the aid is not on motivating smokers to quit smoking (steps $1 \mathrm{t} / \mathrm{m} 4$ of the DGSCC) but rather on the discussion of EBSCls in particular (step 5 of the DGSCC) $(52,53)$. Therefore, instead of focusing on include nonmotivated smokers, an alternative and perhaps more fruitful strategy might be to recruit motivated smokers and keep patients motivated to continue participation, not only in the intervention but also in the entire study (6 months after). Other research found that motivation to quit smoking, indirect recruitment methods, and longer follow-up assessments are associated with a lower retention rate (348). We attempted to increase retention by promising smokers a financial incentive for participating in the study and filling in both questionnaires (baseline and 6 months). Another method for making smoking patients feel that they are participating is by sending them more reminders about their participation in the study (e.g., by making use of social media outlets or newsletters) (64). Another method of recruiting motivated smokers is by attempting to form a personal relationship using a personal approach, thus increasing goodwill with smoking patients (61), such as through scheduling regular follow-up meetings with their PN. Thus, PNs could assume the role of case manager, discussing progress and additional actions when required. In this role, they are also able to intervene earlier when a quit attempt proves unsuccessful. However, due to several factors, such as keeping the workload and questionnaires as brief as possible among others, we were unable to track to what extent these follow-up meetings were executed and what form they took. Toward the end of the main study, the COVID 19 pandemic increased the workload of PCPs further, and therefore, we suspect that this part of the research was not implemented in most cases.

\subsubsection{Facilitating the recruitment of smokers with smoking-related complaints or those from lower SES groups}

As mentioned earlier, smokers with smoking-related complaints often visit the PCS (37, 222); however, this is a difficult population to motivate to quit smoking. Smokers with COPD, for example, experience higher levels of cigarette dependence and depression, while also having lower levels of self-efficacy. They report the same number of quit attempts despite receiving more triggers from their environment to undertake a cessation attempt (e.g., more social support) $(361,367)$. This results in a negative attitude toward smoking cessation among this group, which consequently makes it a frustrating group for PNs to counsel. This can have far-reaching consequences for giving cessation advice and providing additional counseling to this group, such as stigmatization and a lower provision of smoking cessation counseling $(368,369)$. A recent systematic review revealed a combination of mediation with behavioral support to be the only effective treatment (370). To address smokers with smoking-related complaints compared with smokers in the general population, the RA can be adapted for this group by providing extra information on making the most use of a 


\section{CHAPTER 8}

combination of EBSCls or information about any side effects or possible mismatches with commonly prescribed medications. Additional guidance should be provided in the form of specifically targeting their lower levels of self-efficacy, using a social-support group, and adjusting their attitudes toward making another quit attempt.

Because of the preconceived purpose of the study, combined with a strict time period for the development of the RA and the Dutch nationality of the research team, the materials comprising the RA were only available in Dutch. However, as described in Chapter 5 , not all patients had sufficient command of the Dutch language. This might have been a threshold for non-native speakers of Dutch, patients with a migrant background, or lower educated (illiterate) patients. Although these groups of patients often have a lower socioeconomic status (SES), they also suffer from higher smoking rates and encounter more challenges in quitting (16). Potential language barriers also make them a difficult group for PNs to provide counseling to in accordance with the $\operatorname{DGSCC}(52,53)$, and most interventions seem to be effective among higher-SES rather than lower-SES smokers $(371,372)$. We aimed to facilitate this by providing the materials in clear and comprehensible language in accordance with the applicable Dutch guidelines (Language level B1) in order for them to be understandable for less-educated patients.

In conclusion, this section discussed three considerations for recruiting smokers in smoking cessation research and encouraging PNs to keep patients involved in the cessation process, namely (1) using passive recruitment strategies for smokers who have been informed at an earlier time about smoking cessation; (2) keeping the recruitment process of new patients as short as possible (for both PCPs and smokers) and asking for sufficient contact information from the smokers to keep in touch during the trial; and (3) focusing primarily on rerouting motivated smokers and keeping them in the study. Furthermore, the RA can play a role in reaching smokers from lower SES groups, but to recruit them into the study and benefit from the study materials, the materials need to be more customized, both for smokers and PNs, so that they better meet their specific needs and preferences.

\subsection{How to determine smoking cessation effects in research and in practice}

During the RCT, PNs were randomized at the practice level. This meant that participants from the same practice were randomized in the same treatment group to avoid spill-over from the different interventions between patients and different PNs working at the same practice (e.g., patients from the control condition receiving information from the materials distributed in the experimental condition). The power analysis that we conducted to calculate the sample size of the RCT was based on the intra-cluster correlation design mentioned earlier and on a 7-day point prevalence abstinence effect size of $10 \%$ between the control and experimental conditions. However, this design would require a larger sample of smoking participants ( $n=600$ during the recruitment phase, accounting for $50 \%$ dropout 
between recruitment and filling in of the baseline questionnaire, resulting in $n=300$ at baseline; see Chapter 3 for our power analysis), and therefore, would lead to a more complex design, logistics, and analysis. As we only managed to recruit $n=285$ patients, of whom only $n=157$ (55.1\%) filled in the baseline questionnaire during the recruitment period, we could not conduct a cluster randomized trial. It is also possible that selection bias occurred here, as those who agreed to participate in our study might have differed from those who declined to participate, for example, based on their motivation to quit smoking. Furthermore, based on the limited sample, we could not draw significant conclusions on the effects of the RA on smoking abstinence. We also measured decisional conflict to examine whether exposure to the RA may have affected users' decisional process. These two topics are discussed in more detail in the following subsections.

\subsubsection{Measuring smoking abstinence in small samples}

Our RCT found no differences in 7-day point prevalence abstinence and 6-month prolonged abstinence. Given the study design, in which care was provided by a PN in both conditions while it was supplemented by the RA in the experimental condition, whether it was realistic to expect a large difference in smoking cessation rates between the two conditions is questionable. Given the generally low success rates of smoking cessation attempts and to increase the motivation of both PNs and smokers, it may be valuable to reevaluate the measures used for determining when to count a cessation attempt as a (small) success when dealing with a small sample size, or to base effectiveness on reductions in smoking rather than on complete cessation. Future research might therefore consider examining effect measures other than smoking cessation, such as a decrease in the number of cigarettes smoked (per day), decrease in cigarette dependence as assessed via the Fagerström Test for Cigarette Dependence $(208,209)$, multiple serious quit attempts, a shift in willingness ('I intend to quit smoking') or readiness to change ('I intend to quit smoking within 6 months' (e.g. 193, 207), or perhaps even switching to e-cigarettes (fully or slowly phased), some of which have already been found to be prerequisites for a successful quit attempt (373). The change in motivation could be measured over time, for example, during subsequent consultations with their PNs. In the context of this study, the intention to use EBSCls could also be seen as a potential measure of effectiveness, as it was part of one of the aims of the study. By using multiple forms of measurements when evaluating an intervention such as the RA, more information about its overall effectiveness can be distilled. Finally, as the RA is aimed at improving the use of EBSCls, it may also be of particular interest to determine whether its effects are notable among smokers who do not use these ESCBIs. However, this will require a different experimental study.

During our RCT (Chapter 4), all tobacco abstinence measures were assessed through self-reporting as well as with a 'fake lead' question ('Do you object if we come and perform a saliva test to check your smoking status?'). This question would reduce socially desirable 


\section{CHAPTER 8}

responses by incorporating the threat of biochemical testing $(194,195)$. We chose this method of validating abstinence measures rather than biochemical measurements to minimize the effort required for participating in the study for both PNs and smokers. Voluntary appointments by smokers with their PNs are typically used for biochemical validation where a saliva sample is collected using a swab stick to test for the presence of cotinine. However, the response rate to these voluntary appointments in a similar study was low (28), and orchestrating the distribution of cotinine tests, collecting the results, and providing feedback raised doubts about their feasibility. Another way to conduct cotinine research is to collect samples from smokers' homes by, for example, sending a research assistant (69). This would require more sensitive information from participants, which could raise privacy concerns and another barrier to participation. In retrospect, however, both forms of validation would have been impossible because the COVID-19 pandemic overwhelmed PNs and did not allow personal contact. Moreover, previous studies have suggested that the difference between self-reported abstinence rates and those verified with biochemical validation is negligible (196-198). In conclusion, when measuring smoking withdrawal in small samples where contact between the researcher and smokers is minimal, the use of self-reporting is usually sufficient; nevertheless, efforts should be made to objectively measure reductions in smoking behavior or changes in motivation to obtain a full overview of the effectiveness of the intervention.

\subsubsection{Measuring decision making in the PCS}

Inviting smokers to contemplate the optimal or preferred method for quitting smoking rather than (simply) following the advice given in the PCS may in principle lead to increasing uncertainty about the how to quit. To explore the decisional process of choosing a fitting EBSCI option among smokers willing to make a cessation attempt, and also whether the tool facilitated this process, we used the decisional conflict scale (DCS) (334). The DCS measures personal perceptions of uncertainty when choosing options, which includes feelings of being uninformed, being unclear about personal values, and feeling unsupported in the decision-making process. When the decisional conflict measured by the scale is low, there is a higher probability that the decision will be implemented and that the decision maker (i.e., the smoker making a cessation attempt) will be satisfied with their decision. We used the DCS because, in addition to being a validated scale, it takes minimal time and effort to fill in, which agreed with our aim of keeping the study questionnaires as short as possible.

In our main study (Chapter 4), we found no differences between smokers in the experimental and control groups in terms of decisional conflict. Furthermore, both their scores were slightly above the cut-off point, which may indicate some level of conflict associated with decisional delay or uncertainty about implementation (334). In Chapter 7, where we measured decisional conflict among smokers who used the standalone version 
of the RA, decisional conflict was even higher, especially among those who rated the RA as moderately average. As we did not measure decisional conflict at the baseline level, we are unable to say what the actual changes of the RA in decisional conflict were. This means that the level of conflict could also be caused by the expanded supply of EBSCls, or by some forms of EBSCls, their use, and their availability not being sufficiently conveyed to the patients to enable them to make an unconflicted decision. To explore the decision-making process in more detail, further studies should apply more in-depth measures, such as (1) measuring the level of knowledge that smokers gained on the offer of EBSCls after discussing the RA and (2) measuring the perceived autonomy support that smokers experience from their PCP and the RA.

As described in Chapter 1, to make an informed decision, individuals (i.e., smoking patients undertaking a quit attempt) are required to gain information about all relevant details of the EBSCls, such as their cost and effectiveness. To measure whether the individuals succeeded in gaining this information, their knowledge on the subject must be explored. However, no consensus on how to measure knowledge related to decision-making was found in the literature, as there are no cut-off points available for being sufficiently informed $(374,375)$. The Patient Decision Aids Research Group attempted to create a questionnaire for measuring knowledge, which could be used as a template and needs to be tailored to the relevant subject (334). Although the scale has been used in several clinical applications (376-383), to our knowledge it has not yet been used in a setting similar to that of the research described in this dissertation, but it has potential to be adapted to the relevant context (334). Knowledge regarding EBSCls can increase smokers' attitude and self-efficacy regarding the use of EBSCls and might enhance adherence to the chosen form, as they are better informed about how to use EBSCls and what to expect. Measuring this knowledge could also be used to explore whether PNs explain EBSCls in the way that was envisioned in the RA and whether the materials of the RA are sufficiently clear for transferring this knowledge. Therefore, measuring if the RA help ensures a sufficiently high level of knowledge on EBSCls can provide additional information on the operation of the aid.

One of the strengths of the RA, compared with a more traditional DA that is often more focused on standalone use, was the guidance and support that PNs could provide to smokers during the decision-making process. As described in Chapter 1, PNs can take various roles in this process, ranging from more of a guiding role to a role more on the sidelines. The role that PNs might assume depends on the level of autonomy they attribute to their patients or that the patients themselves display. As (perceived) autonomy support has been proven effective in changing health behaviors (such as smoking cessation) (384, 385), the level at which the RA aids the PCP in providing autonomy support can play a role in the overall effectiveness of the RA. During the RCT, we measured the relationship between smoker and PN using the health care climate questionnaire (HCCQ) (386). To further explore the influence of perceived autonomy support and to take the added value of the information 


\section{CHAPTER 8}

of the RA into account, we recommend using a scale such as the Virtual Care Climate Questionnaire (VCCQ), which was developed for web-based health behavior change interventions (387).

In conclusion, to further explore the process of smokers regarding the decision to choose a well-suited EBSCl, measuring knowledge on EBSCls and the degree of autonomy support by both PNs and RA could also be useful options in the decision-making process.

\section{IMPLICATIONS AND RECOMMENDATIONS FOR FUTURE RESEARCH AND PRACTICE}

Since our study was not able to demonstrate the effectiveness of the intervention and the use of the program during the RCT was modest, a recommendation to implement the RA described in this thesis in practice would be misplaced. However, we wish to provide some recommendations for the field of health care, for the RA itself, and for the field of smoking cessation care (specifically in general practice) to strengthen the changes of successful and sustainable development, evaluation, adoption, and implementation in the future.

\subsection{Recommendations for primary care from a PCP perspective}

Previous qualitative findings have suggested that patients with low motivation to quit have a negative impact on PNs' level of self-efficacy, as they view the process of motivating smokers as part of their responsibility $(61,114)$. Although the Dutch smoking cessation guidelines (52) stipulate that all patients be routinely asked if they smoke, this does not always happen $(31,47,49,61)$. Smoking cessation care as part of an intervention or study can be made more efficient by streamlining the actions that PNs are expected to perform and by improving its integration into the PNs' usual routine. Therefore, our recommendations for facilitating research and intervention implementation in primary care are as follows: (1) make better use of co-creation principles during the development and implementation of the intervention and (2) simplify the use of the intervention for PNs to facilitate implementation of the RA. These recommendations are detailed in the following subsections.

\subsubsection{Potentially increasing participation and implementation using successful co-creation} As reported in Chapter 5, we found a low intention to adopt the RA among PNs, even though they reported high appreciation of the materials. This intention was associated with a lower attitude toward the RA (perceiving fewer advantages and more disadvantages), feelings of less social support, and low self-efficacy to use the materials in practice. Other reported barriers were a lack of time and skills. A potential method for motivating PNs to adopt the RA or a related intervention is to attempt to involve them more thoroughly in the process 
of intervention development and implementation. Although we involved potential end-users at different stages of the RA's development, for example, in the form of a Delphi Study (Chapter 2), individual interviews (discussed briefly in Chapter 3), at national congresses in the field of primary care and health promotion, and in testing the materials in a pilot ( $\mathrm{n}$ $\approx 10$, convenience sampling, briefly discussed in Chapter 3 ), the development process began on the basis of scientific research (388). This means that we might not have fully involved all end-users (patients and PNs) and other key stakeholder in the field throughout the process, limiting the effective translation of our research into practice and/or policy (389). This could have been avoided by facilitating more intensive co-creation.

Co-creation focuses on creating value with, and for, multiple stakeholders through regular interactions that can, over time, contribute to the creation of an end product with a high probability of being implemented (390). Research has indicated that implementing co-creation within the development of health-care innovations might (1) decrease the knowledge transition and/or implementation gap (as also described in Chapter 2) (145), (2) enhance involvement in the research, possibly resulting in a higher adherence and recruitment rate (see Chapter 4) (391); and (3) increase the acceptance and intention to adopt a new tool (as discussed in Chapter 5) (392).

However, true co-creation within a time-restricted and regulated sector such as health care is challenging, as PNs often have limited time to partake in tasks that fall outside their daily routine (such as co-creation sessions) (42). To fully incorporate their expertise, the views and experiences of the health-care field need to be adopted as a central starting point. We therefore recommend establishing a fixed group of smoking-cessation experts from both the PCS and research, specifically designed for creating and innovating (smoking cessation) interventions within the PCS. To ensure that a sufficient sense of community is achieved, we suggest involving parties who already have some form of connection or have previously undertaken initiatives together, as was also described in another research study on co-creation in primary care (393).

\subsubsection{Simplifying intervention use for $P N s$ to facilitate implementation}

Despite our efforts to ensure the RA materials' ease of use by providing handouts that summarized the materials and making all materials available online, the RA still contained a large amount of textual information and might have still been too complex to be conveyed to patients during the short time frame of a consultation. Implementing online materials provides more options such as only offering information that is relevant to the individual PN (e.g., (183)) or supplementing with video-based materials that explain the aim and use of the RA, a method that has also been proven to be effective among smoking patients (69), and which may also facilitate reaching higher levels of interactivity (394). In our studies, to spare the PNs as much as possible from completing questionnaires and other means of reporting, we chose not to measure the extent to which PNs went through these materials 


\section{CHAPTER 8}

and understood them, nor did we measure to what extent the materials were applied as intended. The extent to which PNs viewed and understood the prominently paper-based materials within this study therefore remains a black box. It is plausible that poor adherence to and implementation of intervention materials negatively affects a program's impact and possible effectiveness (395). This might not only relate to how the PNs conveyed the materials to their smoking patients during their consultations, but also more importantly to reading and using the materials as they were intended. A possible approach for cracking this black box is to make it easier to measure usage adherence. However, intensively tracking the use of materials, for example, by using triangulated measures (e.g., combining self-reported dose-provided scores of PNs and dose-received measured from a patient perspective) is rather time- and resource-intensive (396). Furthermore, tracking the use of materials can be better facilitated when they are only accessible electronically, for example, by using website tracking technology. Although previous research has reported low adherence when using online materials, another interesting possibility is to examine how, after the COVID-19 pandemic, PNs now view working with online materials, since the pandemic has forced people to be flexible with situations and materials.

As individual interviews with PNs before the development of the RA (briefly reported in Chapter 3) indicated, the PNs often stated that they had little time for peripheral issues not directly related to the delivery of care. Given the relatively high education of the PNs combined with their high workload, when providing the materials for the RA, we had to make a trade-off in terms of providing additional training elements (to elaborate on the use of the RA and to give PNs the option of training their motivational interviewing (MI) techniques; (397)) or explaining the use of the materials as concisely as possible and relying on the knowledge and skills of the users. By applying MI techniques, PNs aim to help patients identify and change unhealthy behaviors through supportive talk therapy based on the principles of cognitive behavioral therapy $(216,398)$. MI has been proven effective in motivating smokers to quit smoking (257), is included in the general educational program that PNs receive (42), and its use is endorsed by the Dutch Guidelines $(52,53)$. Previous research included training that entailed making specific plans for how to best counsel smokers and found it to be effective (252). However, based on the aforementioned tight schedules of the PCP, we did not provide them with comprehensive training regarding the use of the materials or provide extensive MI. Instead, we decided to limit the explanation of how to use the materials to a simple manual of approximately 20 pages, which was also available online, as valuable research has reported that PNs prefer e-learning programs over face-to-face training sessions $(114,399)$. Usually, training courses for this group of people fall under an accreditation program that allows them to complete these hours reimbursement-wise. The design and tight time schedule of this study did not allow us to obtain accreditation. However, previous research has revealed that PNs do not systematically apply these techniques (400-403) and PNs often report 
struggling with motivating smokers to participate in the study. Therefore, in retrospect, it might have been more effective to pay more attention to the role that the RA could play in motivating smokers (i.e., to quit and to partake in the study). We attempted to establish this through stage-based tailoring techniques, outlining different scenarios that PNs could deploy while reaching smokers based on their motivation phase (i.e., not motivated to quit, not motivated but willing to think about quitting, or willing to quit at that moment) (404). Thus, smokers who at the time have no quit intention should first be motivated to quit, while smokers who are already quit-motivated could be motivated to be counseled in accordance with the RA to be motivated to use EBSCIs. Therefore, to help PNs motivate smokers who are unwilling to quit, more attention to $\mathrm{Ml}$ and its techniques could be paid in the RA.

Lastly, providing smoking cessation counseling and referral to EBSCIs, whether as part of research or in daily care, should not feel like a burden. Therefore, instead of opting for more extensive counseling, as is described in the Dutch Guidelines $(52,53)$ and the similar internationally known 5 As (Ask, Advise, Assess, Assist, and Arrange) method of smoking cessation (55), the feasibility and effectiveness of a more brief version of cessation advice for nonmotivated smokers prior to extensive counseling may be explored as well. Research has concluded that brief cessation advice, for example, based on the Ask-Advise-Refer (AAR or the 3 As) (405) or the similar Very Brief Advice (VBA (62, $405)$ ), can result in effectiveness rates similar to those of intensive counseling $(61,64$, 406). Research also found that VBA was also positively received among smokers (407). The AAR strategy focuses less on motivating smokers to quit smoking and more on informing them of the possibilities available to help them when then eventually might be ready to undertake a cessation attempt (407). Because of this setup, this strategy requires less time, knowledge, and skills from the PCP him/herself, but the counseling part is performed by another health-care provider (e.g., a professional smoking cessation counselor) who has more time for it.

Therefore, we suggest that the RA described in this study is implemented in the Refer part of the AAR strategy. This can be done by explaining the available EBSCls to smokers during the referral phase or as a resource that smokers can refer to themselves after talking to their PCP. By implementing the DA in this way, the PCP can save time in counseling smokers who are ready to make a quit attempt. This will leave more time for counseling smokers who are not ready to quit or who need more counseling. Research showed that the number of referrals to EBSCls increased significantly after receiving 3.5 hours of training for a similar method (ABC method, ask-brief advice-cessation support; (406)). When smokers seem receptive to the conversation on smoking cessation, this could then be followed up with the more extensive $5 \mathrm{~A}$ strategy as also described in the Dutch Guidelines $(52,53)$ or another form of EBSCI. 


\section{CHAPTER 8}

\subsection{Recommendations for the RA from a smoker's perspective}

In line with our conclusions from Chapter 7, we also wish to propose some adjustments for making the existing RA more suitable for use as a standalone version, an example of which was described in comparable research (343). These recommendations are as follows: (1) make use of content and frame-tailoring to condense the amount of information and (2) include more informed decision-making principles to help smokers in the decisionmaking process.

Smokers from the usability study described in Chapter 7 indicated that they found the amount of information contained in the RA to be highly extensive. To condense the information presented by the RA and make it more specific to smokers' own preferences and needs, content tailoring (i.e., tailoring the content of the information to the existing knowledge and motivational characteristics of the smoker) can be employed. Content tailoring has already been proven effective in online smoking cessation interventions (341), and adapting the level of counseling to the motivational stage of patients, according to the Stage of Change (110), is recommended by the DGSCC for reaching different groups of smokers $(52,53)$. However, the guideline does not explain how PCPs should adapt their counseling to deal with the differences between the motivational stages of smokers. Previous research (404) found that smokers in the precontemplation stage benefit more from information about the advantages of quitting smoking and the perception of cessation support, whereas smokers in the contemplation and preparation stages benefit more from self-efficacy-enhancing information regarding a cessation attempt; therefore, further research could examine how this could be implemented in the RA.

In addition, content tailoring can be used to make the aid more usable for lower SES groups, such as those who are less educated, as it will enable the fitting of the aid's information to the preferred language. Furthermore, translating the RA's materials, especially those developed to be handed out to patients and the part of the website accessible to patients, into other languages such as Turkish, Arabic, and English - the most-spoken languages in the Netherlands next to Dutch - could assist in reaching these groups of smokers. Tailoring could also be applied to offer a suitable level of complexity for individual users. Thus, smokers who are interested in more in-depth information can obtain it, while smokers who prefer short and simple information will not be put off by long texts and difficult wording. Another communication strategy to reach more lower-educated smokers may be to include more information on video, as previous studies have demonstrated the benefits of video communication over text $(69,345)$.

As mentioned in Subsection 2.3.2, where we discussed our practical considerations regarding the measurement of the aid's decision-making process, and based on our findings in Chapter 7, we recommend paying more attention to supporting smokers in actively considering their own preferences before making a decision for an EBSCI that best fits their needs. As the EBSCls described in this dissertation and in the RA do not differ much in 
effectiveness, cost, or other characteristics, decisions regarding the use of EBSCls can be described as 'preference-sensitive' (127). To structure the preference-sensitive decisionmaking process, value-clarification methods can be used (129). Although the RA already includes a form of implicit value-clarification methods, by inviting users to take in the information and telling them to think it through, the use of explicit forms of value-clarification methods (such as making use of ranking systems to identify preferences) might be more effective (269). Through integrating these methods into the RA, we suspect that smokers would feel more stimulated to actively think about their own preferences for the use of an $\mathrm{EBSCl}$ when they use the intervention on their own.

Naturally, if these adjustments are made to the RA, it will be crucial to pilot test the materials by intensively using a wide range of smoking patients and 'healthy' smokers to ensure an adequate level of usability before testing the intervention by means of a trial.

Lastly, as previously mentioned, if the above-described recommendations were to be included in the DA, exploring the possibility of developing a hybrid variant (blended care; (408)) could be a next step for the RA. Such a variant could be used (1) as a standalone version, (2) to help smokers prepare for a consultation with a PCP, or (3) together with a PCP during counseling sessions. Through this, we would aim to combine the advantages of both faceto-face care and online web-based care $(347,409)$ as patient support offered over the Internet improves patients' self-management, especially when they are appropriately counseled by a PCP $(410,411)$. Another advantage of providing blended care is that it allows the combination of personal attention and synchronous communication with the online advantages of high accessibility $(347,412)$. Given that the PCS prominently reaches smokers who are more motivated to quit $(132,348)$, a mass media approach might reach a broader absolute number of smokers, and even those who are still in a (pre)contemplation phase (132).

Lastly, in Chapter 7, to reach a larger and broader sample of smokers, intention to quit smoking was not an inclusion criterion for participation. This allowed us to evaluate the RA among smokers who had most likely not yet actively thought about a potential cessation attempt and were possibly less informed about EBSCls than smokers with an intention to quit. However, this might also explain the large percentage of smokers who rated the materials as only moderately usable. To reach those smokers who are still contemplating quitting, an additional motivational element would need to be included in the RA, aimed at motivating smokers to quit, helping them set a quit date, and motivating them to use EBSCls during that cessation attempt. Mass media campaigns also make it possible to reach smokers outside of the PCS $(89,132,413)$, who can then use the RA in preparation for a consultation with a PCP. This could reduce the workload of the PCP in question (i.e., the time and energy spent informing smokers about EBSCIs). Whether this would also be a solution for this case as well as how best to combine PCP-led and online patient support should be subjects of future research. Such research should focus on the needs and preferences of smokers regarding the information they require about EBSCls, the talking 


\section{CHAPTER 8}

points they need when discussing EBSCls with the PCP, and how they want to be approached - if they want to be approached - if they do not want to quit immediately. This was seen in a study by Gultzöw et al. ((414)), who investigated smoker profiles and their influence on smokers' intention to use a digital standalone DA.

\subsection{Recommendations for smoking cessation in general}

As described in Chapter 1, tobacco use is a major contributor to smoking-related diseases, health-care costs, and the existing inequality between people of different SES. To counter these negative effects and decrease tobacco use, countless studies have been conducted that have examined the possibilities of illicit structural behavior change in the PCS (e.g., $(31,32))$ and the field of smoking cessation in general (e.g., (28-30, 33-36)). However, most smokers reached by these interventions already had the intention to quit smoking. This was also seen in our main study (Chapter 4). Therefore, as described in earlier research among COPD patients (367), we suspect it would be advantageous to tailor smoking cessation counseling to two groups - motivated smokers and smokers. This means that as long as smokers are not intrinsically motivated to stop smoking (i.e., motivation comes from within the person and is not controlled by an external reward or punishment), they will not be open to counseling or intervention, such as the RA, that can facilitate the smoking cessation process. We suspect that smokers at this stage may also benefit from extrinsic motivators, such as at the macro level. Since tobacco use is recognized as a policy problem by most countries and their governments, the Framework Convention Alliance (FCTC; www.fctc.org) was established $(415,416)$. Countries participating in this Convention are legally obliged to take measures that are in line with the obligations of the FCTC. Policies implemented by the Dutch government under this treaty and the National Prevention Agreement (22) include measures such as more nonsmoking zones, lower availability of cigarettes, and higher costs per pack (cost price and taxes). This strongly underscores the message that smoking has more disadvantages than advantages, which may help smokers to make the switch to a more intrinsic form of motivation. Research has demonstrated that PCPs are also positive about these types of measures, such as price increases and smoking bans, but they feel that their government is not doing enough to reduce smoking and is thus failing to facilitate successful smoking cessation efforts and reduce smoking prevalence (417). The meso level of society (e.g., the workplace) can also play a role, such as through providing financial incentives to motivate smokers to quit smoking, which was found to be effective in a study of employees who received a workplace smoking cessation program (25).

When smokers move from a state of nonmotivation to a more motivated state, either through extrinsic or intrinsic motivation, it is critical that they have access to the care they require to maximize the probability of a successful quit attempt. Increasing the affordability and accessibility of EBSCls will lower the threshold for smokers to make use of them. As 
described earlier, this will also require a proactive move on the side of health-care insurers, who will need to provide more means for both PNs (to provide smoking cessation care to their patients) and smokers (to use EBSCls without financial thresholds). One strategy to consider is increasing attention to policy aspects of smoking cessation care (i.e., reimbursement for providing smoking cessation care to smokers without smoking-related complaints) to ensure a greater chance of successful evaluation, adoption, and implementation of future interventions aimed at increasing the use of EBSCls or stimulating smoking cessation care within the PCS.

Finally, it is better to prevent people starting to smoke than to cure them after they have become addicted. To endorse this, the Dutch 'Toekomst visie huisartsenzorg 2022' (Future vision of general practitioner care 2022) (418) advocates the use of indicated and carerelated prevention. Indicated prevention means targeting individuals who are not officially diagnosed but are at a higher risk (so called 'healthy smokers') to prevent them actually developing smoking-related diseases (419). Care-related prevention targets individuals who developed a disease but aims to support their self-management and thereby reduce their burden and prevent further complications or progression of the disease (419). The RA described in this dissertation could be used for both forms of prevention and, with minor modifications, could tailor the information provided to both groups. Again, since we are talking about people who have already started smoking, from a prevention point of view the best approach is to engage society in universal prevention; that is, targeting people who may not even have started smoking and preventing them from starting (419). The Dutch campaign for the smoke-free generation is an example of this, and given the rising trend of young people still starting to smoke, it is a good way to prevent long-term addictive behavior that is difficult to resolve among the younger generation.

\subsubsection{Availability and accessibility of EBSCls}

A crucial factor in the usability of the RA is the availability of EBSCls. During the main study conducted for this dissertation (described in Chapters 3 and 4), a change occurred in the reimbursement system regarding EBSCls, namely that EBSCIs prescribed by a GP were no longer covered by the deductible. This change in the reimbursement system was initiated by the introduction of the National Prevention Agreement (Nationaal Preventieakkoord (22)) and involves evidence-based forms of behavioral support, NRT, and pharmacotherapy. The use of pharmacotherapy does not count toward the deductible if it is combined with behavioral counseling, which makes it a part of a smoking cessation program, as described in the $\operatorname{DGSCC}(22,52,53)$. This change in policy might lower the financial barriers to the use of EBSCls among smokers. This was also discovered in previous research conducted when EBSCls were first available for reimbursement but still counted against the deductible (94). Although this type of care is reimbursed under the compulsory basic insurance, different conditions apply to each health insurer. The most commonly used policy is the reimbursement of a maximum of one form of behavioral counseling (e.g., individual 


\section{CHAPTER 8}

counseling or group counseling, maximum number of sessions not described in the policy) with the possibility to pair this with a maximum of 3 months use of pharmacotherapy or NRT (as described, for example, on https://www.cz.nl/vergoedingen/stoppen-met-roken). Our recommendation is to further lower the barrier to free smoking cessation by also allowing multiple evidence-based cessation attempts per year, as the cost of EBSCIs could be a barrier to using them for individuals with limited financial resources (420). Doing so is important because the cost of continued smoking is likely to be higher to society and to health insurance than the cost of smoking cessation care.

Although partially forced to by the COVID-19 pandemic, people have started using technology and online services more in their daily lives and work. This has included replacing regular counseling sessions between PNs and smoking patients with (video) calls or email contact. However, the number of effective proven offerings of online smoking cessation care is low and they are often not structurally accessible. For example, although two systematic reviews have been conducted on the effectiveness of eHealth interventions, which identified a total of 121 literature reviews $(421,422)$, a similar review study on the availability of eHealth interventions in the Netherlands (70) reported only six online cessation interventions tested for their effectiveness in trials, none of which are currently widely available. Furthermore, at the time of writing, we could not find any Dutch mHealth interventions that have been tested for effectiveness. Although previous research from 5 years ago found that mHealth apps were rated as potentially inferior to eHealth versions in terms of usability and appreciation (423), times have changed rapidly. A review on this topic did conclude that mHealth interventions are a potentially useful starting point because of the increase in smartphone users (424). Our participants' need to use eHealth (see Chapter 7) calls for a greater supply of validated (i.e., evidence-based, and effective) eHealth interventions. Since there are also numerous Internet interventions available that are not evidence-based (70), the possible introduction of a certification that allows smokers to recognize validated eHealth interventions may further increase their willingness to use eHealth as it will assist their decision-making process.

Another example to illustrate the nonstructurally implemented offerings of EBSCIs is the offering of behavioral counseling in groups. Of the four agencies mentioned in the RA materials, only one offered group therapy at the time of writing. Smokers can choose between online sessions or meetings at a physical location. Physical meetings are only available in five of the 12 Dutch provinces, indicating low accessibility as a result of a long travel time. To provide smokers with a wider range of options with regard to physical group counseling and to facilitate PNs in their referral process, we recommend wider national coverage, as endorsed by the National Prevention Agreement (22). This can be facilitated by involving PNs from (teaching) hospitals, mental health-care institutions (GGZ), and addiction care services to spread the task of providing smoking cessation care. Other relevant stakeholders for developing and maintaining EBSCls are organizations that aim to advance smoking cessation care in Dutch general practice (e.g., Trimbos institute, Quit 
smoking Quality Register, Stop Smoking Partnership or Professional Association of Nurses [Beroepsvereniging Verzorgenden Verpleegkundigen; V\&VN]). Together, they could form a network comparable to the highly effective English network of stop-smoking services (SSSs), which provides both brief cessation counseling and intensive group sessions as well as other forms of behavioral support, possibly supplemented by medication (425). The realization of this transmural cooperation has already been partly set in motion by the renewed version of the care standard for tobacco addiction, which describes how this complex care can be organized within the Dutch care system (426).

\section{GENERAL CONCLUSIONS}

In this dissertation, we aimed to explore the potential of an RA, aiming to increase referral to EBSCls by educating PCPs about the effectiveness of these interventions and facilitating the referral process.

The studies described in this dissertation show that the RA was well appreciated among PCPs and that its use led to slightly more discussion and referral to EBSCls. The RA was also appreciated by smokers, especially those who are motivated to quit smoking. Although the RA introduced a wide range of EBSCls, this did not lead to decisional conflict among its users. However, no effects on smoking abstinence could be reported. This was most probably caused by both conditions receiving evidence-based counseling by their PN and the small study sample. Furthermore, the RA was not intensively used during the RCT, which was probably cased by barriers such as lack of time and a high workload. In this chapter we addressed some considerations regarding facilitating the recruitment of PCPs and smoking patients and their adherence to the RA. We also stated some recommendation for further research related to (1) the PCS (e.g., potentially increasing participation and improving implementation); (2) the RA described in this dissertation (e.g., developing a hybrid variant and supplementing the RA with various forms of motivational techniques, tailoring, video-based materials, and value-clarification exercises); and (3) smoking cessation in general (addressing the health insurance system and policy makers and improving the availability of and access to EBSCIs).

Overall, to increase the use of EBSCIS and implement the RA in the daily routine of PCPS, we can see the potential of the RA as described in this study in the PCs, for example as part of the Ask-Advise-Refer strategy. The available EBSCls should be explained to smokers during the refer phase, or the RA should be used as a reference that smokers can consult themselves after talking to their PCP, which could decrease active counseling time. In order to stimulate implementation, the RA could therefore be included in national smoking cessation trainings aimed at PCPs. Furthermore, the development of a hybrid variant can support the use of the RA in preparation for a consultation with a PCP or allow the smoker to make their own choice regarding the use of EBSCls in case the tool is offered online as a self-help tool. 
APPENDICES 
Impact paragraph Abbreviation list References

Summary

Samenvatting

Curriculum vitae

Publication list

Dankwoord 


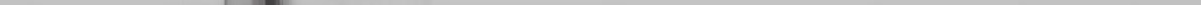




\section{IMPACT PARAGRAPH}

Research is only effective and can have an impact when its results can be used, either in practice or as a springboard, for further research. The aim of the studies described in this dissertation was to increase the referral of smoking patients to EBSCls. We wanted to achieve this by educating PCPs on the availability, usability, and effectiveness of EBSCIs and by facilitating the referral process to EBSCIs. We therefore developed a referral aid (RA) called the "StopWijzer" (which can be translated as "Stop guide" or "Stop smarter"). The referral aid (RA) did not change smoking cessation rates - which may be due to the fact that in both conditions effective care was provided by the PCPs and to the small sample size in the randomized controlled trial. The results did show that motivated smokers appreciated the RA suggesting that for implementation of such tools may a relevant impact on smokers motivated to quit to aid smoking cessation decision making.

\section{Research aim}

Our research showed us that PCPs expressed interested in receiving and using an overview of available EBSCIs, becaused they felt that they lacked knowledge on the subject, but also that they had little time in their counseling sessions to provide extensive counseling (Chapter 2). However, PCPs' use of the RA during our randomized controlled trial was low (described in chapter 3 ), resulting in a low recruitment rate of smoking patients (chapter 4). Therefore, we were unable to identify effects of the RA on smoking abstinence. Despite low adherence and high rate of attrition, the RA was received well by both PCPs and smoking patients and there was a trend toward more referral and use of EBSCls in the experimental condition. An additional study on the intention to adopt the RA among PCPs, as described in Chapter 5, confirmed this positive appreciation of the RA. However, it also revealed that a large proportion of the PCPs surveyed had no intention of adopting the RA, which was influenced by a more negative attitude toward the advantages of the RA caused by lower self-efficacy and perceived barriers such as a lack of time. Further research suggested that the use of (decision) aids that aim to facilitate the decision-making process underlying the choice between several EBSCls has the potential to bring about behavior change (i.e., smoking cessation - Chapter 6). Lastly, we tested the usability of an adapted standalone version of the RA among a group larger group of smokers (see Chapter 7). Most participants found the DA only moderately usable, though those who found it more usable often had a higher intention to quit. Based on the results of that study, recommendations to implement the RA for smokers motivated to quit were made. Additionally, recommendations to make the materials more usable and valuable for smokers not motivated to quite were made, such as motivational techniques, tailoring, using video-based information, and including value clarification methods. Furthermore, a hybrid variant was suggested where smokers could use the DA 


\section{APPENDICES}

independently and under the guidance of a PCP, which could aid both groups to choose an appropriate EBSCI option. Further research is needed to explore the possibilities of such a hybrid variant.

\section{Relevance of the results \\ Practical relevance}

First, as the RA was appreciated by smokers motivated to quit, implementation of such a guide may help this group to aid smoking cessation decision making. Second, the RA may also help PCPs to identify EBSCl's and to attune them smokers' needs. Yet, as its use in our RCT was still low, additional studies are needed on how to improve the RAs use in practice, e.g., by also including the RA in regular smoking cessation training programs. Third, as the RA was not optimally appreciated by smokers not motivated to quit, additional strategies to optimize the RA may be needed, such as including motivational elements tailored to the preferences and needs of this specific group.

\section{Scientific relevance}

We hoped that use of the RA could improve smoking cessation rates. Yet, we did not find this, potentially also because current care in both conditions of PCPs were sufficient. Testing the efficacy of the RA among those not using EBSCI's might be a next step, as one would hope that usage of the RA would lead to more use of EBSCIs. We also aimed to determine the potential factors facilitating and hindering the effectiveness and possible adoption and implementation of the RA. Although we were only able to provide some preliminary insights into the effects of the RA on smoking abstinence and EBSCI use, we were able to formulate conclusions about factors relevant for the daily practice in the PCS (e.g., the facilitators and barriers for using a DA within the limited timeframe of a counseling session). We also explored the possibilities of further developing the RA to make it possible to use it as a standalone version or as a part of 'blended care' (i.e., a combination of face-to-face counseling and web-based care), which can be used to reach a broader range of smokers willing to quit smoking.

\section{Societal relevance}

As tobacco use increases the risk of developing cancer and cardiovascular and pulmonary diseases, the burden of smoking in society remains enormous. The RA is of societal relevance, as it may help motivated smokers to find the most effective and preferred method to quit smoking, both among smokers individually as well as among smokers quitting with the aid of professionals. Additionally, it is of relevance as it provides PCPs a concise way to identify EBSCIs. Implementation of such tools in training programs of PCP's is thus recommended in order to facilitate identifying the relevant EBSCl's for smokers in their practice. By also paying special attention to groups with smoking-related complaints (which 
accounted for approximately $50 \%$ of our sample), we aimed to increase smoking cessation success rates in this group, thus contributing to the decrease of the health divide in society. Yet, in order to optimize societal use, further implementation strategies are needed to target more smokers, also those who are unmotivated to quit yet to quit smoking, to increase societal impact.

\section{Involving target groups}

We identified three major target groups who can draw lessons from the outcomes of our study: (1) the PCS, as represented by PCPs; (2) the research field of smoking cessation care in the PCS, as represented by scientific researchers; and (3) society, as represented by policy makers in the field of prevention and professionals in the field of health insurance.

\section{Involving the primary care setting}

The PCS plays an important role in providing smoking cessation care at the individual level. Not only does the PCS have a wide reach but also a unique position, possessing the skills and knowledge to offer smokers the support they need to undertake a successful cessation attempt. Here, the first step is asking each patient whether they smoke (ask), advising smokers to quit (advice), and providing support to smokers who want to undertake a quit attempt (refer) based on the Dutch guidelines for smoking cessation care in the PCS. The RA described in this dissertation played a role in the referral part of this strategy by providing PCPs with an overview of the available EBSCls. PCPs should also be aware of the vital role they play in engaging smokers with a lower SES or smoking-related complaints, as these group often have lower self-efficacy to ask for help.

To embed the findings of our research project within the PCS in a way that optimizes its impact, the active involvement of potential end-users during all phases of the development and diffusion of an intervention is required to ensure feasibility and effectiveness. Although we tried to include the PCS by using principles of co-creation (i.e., one-on-one interviews briefly mentioned in Chapter 3 and the Delphi study described in Chapter 2), to truly explore PCPs' needs and potential facilitators and barriers relevant for the RA, a more bottom-up approach may be needed. To enable true co-creation in further research, the constitution of a smoking cessation care working group may be considered. This group should include multiple potential end-users from the PCS, such as general practitioners and PNs, practice managers, and policy advisors from a wide array of PCSs, who should be involved in the various research phases whenever possible. Involving endusers from the start of a project would not only help to bridge the gap between daily practice and scientific research but may also facilitate motivation to adopt or to participate in associated research. 


\section{APPENDICES}

\section{Involving the scientific community}

To increase scientific impact, it is important to reach smoking cessation researchers in general and those in the field of smoking cessation care based in the PCS (i.e., those who develop smoking cessation interventions or guidelines with the aim of improving smoking cessation care in the PCS). At the time of writing, all studies included in this thesis had been submitted to or published in international peer-reviewed journals. Some findings described in this dissertation have also been presented and discussed at (inter)national congresses focused on smoking cessation, primary care, or decision making. Naturally, we will continue to try to involve researchers in the field of smoking cessation by reporting the findings of our studies via peer-reviewed and - when possible - open-access research journals. We recommend the field of research to actively look for solutions for the discovered barriers in this field. In addition to interventions targeting the PCS, we should also aim to increase the amount of evidence-based and structurally available EBSCIs, especially in the form of eHealth and mHealth.

Furthermore, the RA described in this dissertation and the insights provided by our research can perhaps be useful for the developers of the (Dutch) smoking cessation guidelines for the PCS. Although the guidelines currently recommend referring smokers to EBSCls, they do not specify how this can be done in effectively and efficiently. The RA described in this dissertation can be used to improve the information provided by the guidelines or as a foundation for more specific information dissemination.

\section{Involving society}

The last important group that needs to be included to make the RA more suitable for widespread implementation in daily practice, is policy makers in the field of prevention and health promotion and professionals in the field of health insurance. As the Dutch proverb goes, prevention is better than cure. Next to preventing the younger generation to start smoking, the measures described in the National Prevention Agreement are mostly aimed at discouraging smoking among existing 'healthy' smokers to prevent them from developing smoking-related complaints. Smokers who seek to quit smoking, including those who are still relatively healthy (i.e., have not yet developed smoking-related complaints), should be able to receive smoking cessation aid, either as counseling or in a different form, as also endorsed by the RA. Policy makers should aim to facilitate smoking cessation counseling in the PCS by, for example, increasing reimbursements for counseling smoking patients, even when they do not have smoking-related complaints. To lower the threshold for a successful quit attempt even further, smokers should be provided with unlimited access to EBSCls without it having to count towards the yearly deductible set by health insurers. Therefore, we recommend full reimbursement of evidence-based smoking cessation care and interventions to improve access to evidence-based help to quit and increase the use of EBSCls when undertaking a cessation attempt. 
In conclusion, our RA is one of the first attempts to guide both PCPs and smokers in identifying the optimal smoking cessation strategy for the smoker to quit smoking. Whereas the RA (also) targeted smokers who are not highly motivated to quit, it may be more practical and effective to use the RA for smokers who are motivated to quit. Despite indications showing positive evaluations concerning the RA's usability, strategies for identifying factors that facilitate its use by PCPs and smokers are essential to be able to demonstrate the beneficial effects of the use of such RAs on smoking cessation rates. 


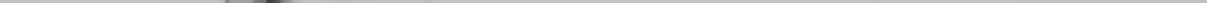




$\begin{array}{ll}\text { ABBREVIATION LIST } \\ \text { DA } & \text { decision aid } \\ \text { DGSCC } & \text { Dutch Smoking Cessation Guidelines } \\ \text { DRQSCP } & \text { Dutch Register for Qualified Smoking Cessation Professionals } \\ & \text { Kwaliteitsregister Stoppen met Roken } \\ \text { EBSCI } & \text { evidence-based smoking cessation intervention } \\ \text { GP } & \text { general practitioner } \\ \text { HCPs } & \text { health care professionals } \\ \text { ICM } & \text { integrated change model } \\ \text { NRT } & \text { nicotine replacement therapy } \\ \text { PCPS } & \text { primary care professionals } \\ \text { PCS } & \text { primary care setting } \\ \text { PN } & \text { practice nurse } \\ \text { RA } & \text { referral aid } \\ \text { RCT } & \text { randomized controlled trail } \\ \text { SCC } & \text { smoking cessation counseling } \\ \text { SCI } & \text { smoking cessation interventions } \\ \text { SES } & \text { social economic status } \\ \text { TPB } & \text { theory of planned behavior }\end{array}$




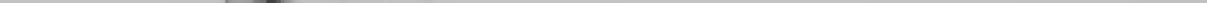




\section{REFERENCES}

1. Charlton A. Tobacco or health 1602: an Elizabethan doctor speaks. Health Educ Res. 2005;20(1):101-11.

2. Hill J. Cautions against the immoderate use of snuff. Founded on the known qualities of the tobacco plant; and the effects it must produce when this way taken into the body: R. Baldwin; J. Jackson; 1761.

3. Bayne-Jones S. Smoking and health: report of the Advisory Committee to the Surgeon General of the public health service. US Department of Health, Education, and Welfare, Public Health Service; 1964.

4. Meinsma L. Roken en risico's. Lochem, the Netherlands: De Tijdstroom; 1969.

5. Henningfield JE, Fagerstrom KO. Swedish Match Company, Swedish snus and public health: a harm reduction experiment in progress? Tob Control. 2001;10(3):253-7.

6. World Health Organization. WHO report on the global tobacco epidemic 2019: Offer help to quit tobacco use. 2019.

7. Eurobarometer. Attitudes of Europeans towards tobacco and electronic cigarettes. 2015.

8. Bruggink J-W. Ontwikkelingen in het aandeel rokers in Nederland sinds 1989. Tijdschrift voor gezondheidswetenschappen. 2013;91(4):234-40.

9. Bommele J, Willemsen M. Kerncijfers roken 2020. Trimbos-instituut: Utrecht, The Netherlands. 2021.

10. National Center for Chronic Disease Prevention and Health Promotion (US) Office on Smoking and Health. The health consequences of smoking -50 years of progress: a report of the Surgeon General. Atlanta: Centers for Disease Control and Prevention; 2014.

11. Polder JJ, van Gils PF, Kok L, Talhout R, Feenstra T. De rekening van roken. Tijdschrift voor Geneeskunde. 2017;161:D833.

12. Verdurmen J, Monshouwer K, Van Laar M, Van Bon-Martens M. Factsheet continu onderzoek rookgewoonten 2013. Utrecht: Trimbos-instituut; 2014.

13. Willemsen MC. Tobacco control policy in the Netherlands: between economy, public health, and ideology: Springer Nature; 2018.

14. Hilderink H, Verschuuren M. Volksgezondheid Toekomst Verkenning 2018: Een gezond vooruitzicht. Synthese. Bilthoven: Rijksinstituut voor Volksgezondheid en Milieu; 2018.

15. Nagelhout GE, de Vries H, Fong GT, Candel MJ, Thrasher JF, van den Putte B, et al. Pathways of change explaining the effect of smoke-free legislation on smoking cessation in The Netherlands. An application of the international tobacco control conceptual model. Nicotine \& tobacco research : official journal of the Society for Research on Nicotine and Tobacco. 2012;14(12):1474-82.

16. Hiscock R, Bauld L, Amos A, Fidler JA, Munafo M. Socioeconomic status and smoking: a review. Ann N Y Acad Sci. 2012;1248(1):107-23.

17. Hummel K, Willemsen MC, de Vries H, Monshouwer K, Nagelhout GE. Social Acceptance of Smoking Restrictions During 10 Years of Policy Implementation, Reversal, and Reenactment in the Netherlands: Findings From a National Population Survey. Nicotine \& tobacco research : official journal of the Society for Research on Nicotine and Tobacco. 2017;19(2):231-8.

18. Heijndijk S, Willemsen M. Dutch tobacco control: Moving towards the right track? FCTC shadow report 2014. Dutch Alliance for a Smokefree Society, The Hague, The Netherlands; 2015.

19. Verdonk-Kleinjan WM, Knibbe RA, Tan FE, Willemsen MC, de Groot HN, de Vries H. Does the workplacesmoking ban eliminate differences in risk for environmental tobacco smoke exposure at work? Health Policy. 2009;92(2-3):197-202.

20. Verdonk-Kleinjan WM, Rijswijk PC, Candel MJ, de Vries H, Knibbe RA. Agreement between self-reports and on-site inspections of compliance with a workplace smoking ban. Nicotine \& Tobacco Research. 2012;14(9):1121-5.

21. Gonzalez M, Glantz SA. Failure of policy regarding smoke-free bars in the Netherlands. The European Journal of Public Health. 2013;23(1):139-45.

22. Ministerie van Volkgezondheid Welzijn en Sport. Nationaal Preventieakkoord: Naar een gezonder Nederland. Den Haag: Ministry of Public Health, Welfare and Sport; 2018.

23. van Bladeren F, Muller G. Toward a Smoke-free Generation: The Dutch Strategy. American Society of Clinical Oncology; 2018.

24. Troelstra SA, Harting J, Kunst AE. Effectiveness of a Large, Nation-Wide Smoking Abstinence Campaign in the Netherlands: A Longitudinal Study. Int J Environ Res Public Health. 2019;16(3):378. 


\section{APPENDICES}

25. van den Brand FA, Nagelhout GE, Winkens B, Chavannes NH, van Schayck OCP. Effect of a workplace-based group training programme combined with financial incentives on smoking cessation: a cluster-randomised controlled trial. Lancet Public Health. 2018;3(11):e536-e44.

26. Cahill K, Lancaster T. Workplace interventions for smoking cessation. Cochrane Database Syst Rev. 2014(2):CD003440.

27. Fishwick D, Carroll C, McGregor M, Drury M, Webster J, Bradshaw L, et al. Smoking cessation in the workplace. Occupational medicine (Oxford, England). 2013;63(8):526-36.

28. Smit ES, de Vries $\mathrm{H}$, Hoving $\mathrm{C}$. The PAS study: a randomized controlled trial evaluating the effectiveness of a web-based multiple tailored smoking cessation programme and tailored counselling by practice nurses. Contemporary clinical trials. 2010;31(3):251-8.

29. Reinwand DA, Crutzen R, Elfeddali I, Schneider F, Schulz DN, Smit ES, et al. Impact of educational level on study attrition and evaluation of web-based computer-tailored interventions: results from seven randomized controlled trials. Journal of Medical Internet Research. 2015;17(10):e228.

30. Stanczyk NE, Bolman C, Muris JW, de Vries H. Study protocol of a Dutch smoking cessation e-health program. BMC Public Health. 2011;11(1):847.

31. de Ruijter D, Candel M, Smit ES, de Vries H, Hoving C. The effectiveness of a computer-tailored e-learning program for practice nurses to improve their adherence to smoking cessation counseling guidelines: randomized controlled trial. Journal of medical Internet research. 2018;20(5):e193.

32. Leitlein L, Smit ES, de Vries H, Hoving C. Factors influencing Dutch practice nurses' intention to adopt a new smoking cessation intervention. J Adv Nurs. 2012;68(10):2185-94.

33. Segaar D, Willemsen MC, Bolman C, De Vries H. Nurse adherence to a minimal-contact smoking cessation intervention on cardiac wards. Res Nurs Health. 2007;30(4):429-44.

34. Berndt N, Bolman C, Lechner L, Mudde A, Verheugt FW, de Vries H. Effectiveness of two intensive treatment methods for smoking cessation and relapse prevention in patients with coronary heart disease: study protocol and baseline description. BMC cardiovascular disorders. 2012;12(1):33.

35. Berndt N, Bolman C, Froelicher ES, Mudde A, Candel M, de Vries H, et al. Effectiveness of a telephone delivered and a face-to-face delivered counseling intervention for smoking cessation in patients with coronary heart disease: a 6-month follow-up. J Behav Med. 2014;37(4):709-24.

36. Meijer E, van der Kleij R, Segaar D, Chavannes N. Determinants of providing smoking cessation care in five groups of healthcare professionals: A cross-sectional comparison. Patient Educ Couns. 2019;102(6):1140-9.

37. Kleinjan M, Bommelé J, Verdurmen J, Van Laar M. Het bespreken van (stoppen met) roken door de huisarts en andere zorgverleners (tandartsen, medisch specialisten en verloskundigen). Utrecht: Trimbos-instituut; 2016.

38. Wendel-Vos G, Picavet H, van Gelder B, Tijhuis M, Droomers M. Meervoudig ongezond gedrag in Nederland: Een exploratie van risicogroepen en samenhang met omgeving, gezondheid en zorggebruik. Bilthoven: RIVM; 2008.

39. Centraal Bureau voor Statistiek. Zorggebruik verschilt per opleidingsniveau: CBS; 2014 [Available from: https://www.cbs.nl/nl-nl/nieuws/2014/46/zorggebruik-verschilt-per-opleidingsniveau.

40. Control CfD, Prevention. Physician and other health-care professional counseling of smokers to quit--United States, 1991. 1993. Report No.: 0149-2195 Contract No.: 44.

41. van Beek A. Ontstaan en ontwikkeling van de praktijkondersteuning. Tijdschrift voor praktijkondersteuning. 2013;8(2):48-50.

42. Heiligers P, Noordman J, Korevaar J, Dorsman S, Hingstman L, Van Dulmen A, et al. Kennisvraag: praktijkondersteuners in de huisartspraktijk (POH's), klaar voor de toekomst? Utrecht: NIVEL; 2012.

43. Pieterse ME, Seydel E, Wiegman O. Smoking cessation via the general practitioner: Development of a minimal contact intervention programme. Patient education and counseling. 1992;19(1):107-8.

44. Pieterse ME, Seydel ER, DeVries H, Mudde AN, Kok GJ. Effectiveness of a minimal contact smoking cessation program for Dutch general practitioners: a randomized controlled trial. Prev Med. 2001;32(2):182-90.

45. Hilberink SR, Jacobs JE, Bottema BJ, de Vries H, Grol RP. Smoking cessation in patients with COPD in daily general practice (SMOCC): six months' results. Prev Med. 2005;41(5-6):822-7.

46. de Vries H, Bakker M, Mullen PD, Van Breukelen $\mathrm{G}$. The effects of smoking cessation counseling by midwives on Dutch pregnant women and their partners. Patient Education and Counseling. 2006;63(1-2):177-87.

47. Segaar D, Bolman C, Willemsen M, De Vries H. Identifying determinants of protocol adoption by midwives: a comprehensive approach. Health Educ Res. 2007;22(1):14-26. 
48. Segaar D, Bolman C, Willemsen MC, Vries H. Determinants of adoption of cognitive behavioral interventions in a hospital setting: example of a minimal-contact smoking cessation intervention for cardiology wards. Patient Educ Couns. 2006;61(2):262-71.

49. Berndt N, Lechner L, De Vries H, Van Acker F, Mudde A, Froelicher E, et al. Effectiveness of two intensive smoking cessation interventions for the secondary prevention of coronary heart disease: benefits for patients with low socioeconomic status and low quit motivations. European Heart Journal. 2013;34(suppl 1):P2529-P.

50. Siemer L, Pieterse ME, Brusse-Keizer MG, Postel MG, Ben Allouch S, Sanderman R. Study protocol for a non-inferiority trial of a blended smoking cessation treatment versus face-to-face treatment (LiveSmokefreeStudy). BMC Public Health. 2016;16(1):1187.

51. Jacobs-van der Bruggen M, Donker G, Verkleij H, Baan C. Stoppen met roken: hoe pakken wij dat aan? Huisarts en wetenschap. 2007;50(5):330-5.

52. Chavannes N, Drenthen T, Wind L, Van Avendonk M, Van den Donk M, Verduijn M. NHG-Behandelrichtlijn Stoppen met roken. Utrecht: Nederlands Huisartsen Genootschap. 2017.

53. Stop Smoking Partnership. Guideline treatment tobacco addiction [richtlijn behandeling van tabaksverslaving]. Alphen aan den Rijn: Stop Smoking Partnership; 2019.

54. de Ruijter D, Smit ES, de Vries H, Hoving C. Dutch practice nurses' adherence to evidence-based smoking cessation treatment guidelines. Fam Pract. 2017;34(6):685-91.

55. Fiore MC, Jaén CR, Baker TB, Bailey WC, Benowitz NL, Curry SJ, et al. Treating tobacco use and dependence: 2008 update. 2008.

56. Rice VH, Stead LF. Nursing interventions for smoking cessation. Cochrane Database Syst Rev. 2008(1):CD001188.

57. Stead LF, Buitrago D, Preciado N, Sanchez G, Hartmann-Boyce J, Lancaster T. Physician advice for smoking cessation. Cochrane Database Syst Rev. 2013(5):CD000165.

58. Studts JL, Flynn SM, Dill TC, Ridner SL, Worth CT, Walsh SE, et al. Nurse practitioners' knowledge, attitudes, and clinical practices regarding treatment of tobacco use and dependence. The Journal for Nurse Practitioners. 2010;6(3):212-9.

59. Walters EL, Reibling ET, Wilber ST, Sullivan AF, Gaeta TJ, Camargo CA, Jr., et al. Emergency department provider preferences related to clinical practice guidelines for tobacco cessation: a multicenter survey. Academic emergency medicine : official journal of the Society for Academic Emergency Medicine. 2014;21(7):785-93.

60. Verbiest M, Brakema E, van der Kleij R, Sheals K, Allistone G, Williams S, et al. National guidelines for smoking cessation in primary care: a literature review and evidence analysis. NPJ Prim Care Respir Med. 2017;27(1):2.

61. van Rossem C, Spigt MG, Kleijsen JR, Hendricx M, van Schayck CP, Kotz D. Smoking cessation in primary care: Exploration of barriers and solutions in current daily practice from the perspective of smokers and healthcare professionals. Eur J Gen Pract. 2015;21(2):111-7.

62. Aveyard P, Begh R, Parsons A, West R. Brief opportunistic smoking cessation interventions: a systematic review and meta-analysis to compare advice to quit and offer of assistance. Addiction. 2012;107(6):1066-73.

63. Papadakis S, Cole AG, Reid RD, Coja M, Aitken D, Mullen KA, et al. Increasing Rates of Tobacco Treatment Delivery in Primary Care Practice: Evaluation of the Ottawa Model for Smoking Cessation. Ann Fam Med. 2016;14(3):235-43.

64. van Rossem C, Spigt M, Viechtbauer W, Lucas AE, van Schayck OC, Kotz D. Effectiveness of intensive practice nurse counselling versus brief general practitioner advice, both combined with varenicline, for smoking cessation: a randomized pragmatic trial in primary care. Addiction. 2017;112(12):2237-47.

65. Verbiest ME, Chavannes NH, Crone MR, Nielen MM, Segaar D, Korevaar JC, et al. An increase in primary care prescriptions of stop-smoking medication as a result of health insurance coverage in the Netherlands: population based study. Addiction. 2013;108(12):2183-92.

66. Rice VH, Heath L, Livingstone-Banks J, Hartmann-Boyce J. Nursing interventions for smoking cessation. Cochrane Database Syst Rev. 2017;12(12):CD001188.

67. de Josselin de Jong S, Candel M, Segaar D, Cremers HP, de Vries H. Efficacy of a Web-based computertailored smoking prevention intervention for Dutch adolescents: randomized controlled trial. J Med Internet Res. 2014;16(3):e82.

68. Elfeddali I, Bolman C, Candel MJ, Wiers RW, de Vries H. Preventing smoking relapse via Web-based computertailored feedback: a randomized controlled trial. J Med Internet Res. 2012;14(4):e109.

69. Stanczyk N, Bolman C, van Adrichem M, Candel M, Muris J, de Vries H. Comparison of text and video computer-tailored interventions for smoking cessation: randomized controlled trial. J Med Internet Res. 2014;16(3):e69. 


\section{APPENDICES}

70. Cheung KL, Wijnen B, de Vries H. A Review of the Theoretical Basis, Effects, and Cost Effectiveness of Online Smoking Cessation Interventions in the Netherlands: A Mixed-Methods Approach. J Med Internet Res. 2017;19(6):e230.

71. Taylor GMJ, Dalili MN, Semwal M, Civljak M, Sheikh A, Car J. Internet-based interventions for smoking cessation. Cochrane Database Syst Rev. 2017;9(9):CD007078.

72. Matkin W, Ordonez-Mena JM, Hartmann-Boyce J. Telephone counselling for smoking cessation. Cochrane Database Syst Rev. 2019;5(5):CD002850.

73. Stead LF, Carroll AJ, Lancaster T. Group behaviour therapy programmes for smoking cessation. Cochrane Database Syst Rev. 2017;3(3):CD001007.

74. McEwen A, West R, McRobbie H. Effectiveness of specialist group treatment for smoking cessation vs. oneto-one treatment in primary care. Addict Behav. 2006;31(9):1650-60.

75. Hurt RD, Sachs DP, Glover ED, Offord KP, Johnston JA, Dale LC, et al. A comparison of sustained-release bupropion and placebo for smoking cessation. The New England journal of medicine. 1997;337(17):1195202.

76. Hughes JR, Stead LF, Lancaster T. Nortriptyline for smoking cessation: a review. Nicotine \& tobacco research : official journal of the Society for Research on Nicotine and Tobacco. 2005;7(4):491-9.

77. Tonstad S, Tonnesen P, Hajek P, Williams KE, Billing CB, Reeves KR, et al. Effect of maintenance therapy with varenicline on smoking cessation: a randomized controlled trial. Jama. 2006;296(1):64-71.

78. Stead LF, Perera R, Bullen C, Mant D, Hartmann-Boyce J, Cahill K, et al. Nicotine replacement therapy for smoking cessation. Cochrane Database Syst Rev. 2012;11:CD000146.

79. Lindson N, Chepkin SC, Ye W, Fanshawe TR, Bullen C, Hartmann-Boyce J. Different doses, durations and modes of delivery of nicotine replacement therapy for smoking cessation. Cochrane Database Syst Rev. 2019;4(4):CD013308.

80. Hughes JR, Stead LF, Hartmann-Boyce J, Cahill K, Lancaster T. Antidepressants for smoking cessation. Cochrane Database Syst Rev. 2014(1):CD000031.

81. Cahill K, Lindson-Hawley N, Thomas KH, Fanshawe TR, Lancaster T. Nicotine receptor partial agonists for smoking cessation. Cochrane Database Syst Rev. 2016(5):CD006103.

82. Kotz D, Fidler JA, West R. Did the introduction of varenicline in England substitute for or add to the use of other smoking cessation medications? Nicotine \& Tobacco Research. 2011;13(9):793-9.

83. Stead LF, Koilpillai P, Fanshawe TR, Lancaster T. Combined pharmacotherapy and behavioural interventions for smoking cessation. Cochrane Database Syst Rev. 2016;3(3):CD008286.

84. Lancaster T, Stead LF. Individual behavioural counselling for smoking cessation. Cochrane Database Syst Rev. 2017;3(3):CD001292.

85. White AR, Rampes H, Liu JP, Stead LF, Campbell J. Acupuncture and related interventions for smoking cessation. Cochrane Database Syst Rev. 2011(1):CD000009.

86. West R, Raw M, McNeill A, Stead L, Aveyard P, Bitton J, et al. Health-care interventions to promote and assist tobacco cessation: a review of efficacy, effectiveness and affordability for use in national guideline development. Addiction. 2015;110(9):1388-403.

87. Zhu S-H, Melcer T, Sun J, Rosbrook B, Pierce JP. Smoking cessation with and without assistance: a populationbased analysis. American journal of preventive medicine. 2000;18(4):305-11.

88. Filippidis FT, Laverty AA, Mons $\mathrm{U}$, Jimenez-Ruiz $\mathrm{C}$, Vardavas $\mathrm{Cl}$. Changes in smoking cessation assistance in the European Union between 2012 and 2017: pharmacotherapy versus counselling versus e-cigarettes. Tobacco control. 2019;28(1):95-100.

89. Borland R, Li L, Driezen P, Wilson N, Hammond D, Thompson ME, et al. Cessation assistance reported by smokers in 15 countries participating in the International Tobacco Control (ITC) policy evaluation surveys. Addiction. 2012;107(1):197-205.

90. Hummel K, Nagelhout GE, Fong GT, Vardavas Cl, Papadakis S, Herbeć A, et al. Quitting activity and use of cessation assistance reported by smokers in eight European countries: Findings from the EUREST-PLUS ITC Europe Surveys. Tobacco induced diseases. 2018;16.

91. Willemsen MC, Wiebing M, van Emst A, Zeeman G. Helping smokers to decide on the use of efficacious smoking cessation methods: a randomized controlled trial of a decision aid. Addiction. 2006;101(3):441-9.

92. Marques-Vidal P, Melich-Cerveira J, Paccaud F, Waeber G, Vollenweider P, Cornuz J. High expectation in nonevidence-based smoking cessation interventions among smokers--the CoLaus study. Prev Med. 2011;52(34):258-61. 
93. Willemsen MC, Segaar D, van Schayck OC. Population impact of reimbursement for smoking cessation: a natural experiment in the $\mathrm{N}$ etherlands. Addiction. 2013;108(3):602-4.

94. Nagelhout GE, Willemsen MC, van den Putte B, de Vries H, Willems RA, Segaar D. Effectiveness of a national reimbursement policy and accompanying media attention on use of cessation treatment and on smoking cessation: a real-world study in the Netherlands. Tob Control. 2015;24(5):455-61.

95. Rijksoverheid. Kan ik hulp krijgen als ik wil stoppen met roken? 2021 [Available from: https://www.rijksoverheid.nl/onderwerpen/roken/vraag-en-antwoord/kan-ik-hulp-krijgen-als-ik-wil-stoppen-met-roken.

96. Springvloet L, Van Laar M. Roken onder volwassenen: kerncijfers 2016. Utrecht: Nationaal Expertisecentrum Tabaksontmoediging; 2017.

97. Vogt F, Hall S, Marteau TM. Understanding why smokers do not want to use nicotine dependence medications to stop smoking: qualitative and quantitative studies. Nicotine \& Tobacco Research. 2008;10(8):1405-13.

98. Vogt F, Hall S, Marteau TM. Examining why smokers do not want behavioral support with stopping smoking. Patient education and counseling. 2010;79(2):160-6.

99. Weinstein ND, Slovic P, Gibson G. Accuracy and optimism in smokers' beliefs about quitting. Nicotine \& Tobacco Research. 2004;6(Suppl_3):S375-S80.

100. Hammond D, McDonald P, Fong G, Borland R. Do smokers know how to quit? Knowledge and perceived effectiveness of cessation assistance as predictors of cessation behaviour. Addiction. 2004;99(8):1042-8.

101. Bommele J, Willemsen M. Kerncijfers roken 2018. Trimbos-instituut: Utrecht, The Netherlands. 2019.

102. Trimbos-instituut. Stoppen met roken: feiten en cijfers 2018 [Available from: https://www.trimbos.nl/kennis/ stoppen-met-roken/stoppen-met-roken-feiten-en-cijfers.

103. Friedrichs A, Spies $M$, Härter $M$, Buchholz A. Patient preferences and shared decision making in the treatment of substance use disorders: a systematic review of the literature. PloS one. 2016;11(1):e0145817.

104. Hoving C, Visser A, Mullen PD, van den Borne B. A history of patient education by health professionals in Europe and North America: from authority to shared decision making education. Patient Educ Couns. 2010;78(3):275-81.

105. de Vries $\mathrm{H}$. An integrated approach for understanding health behavior; the I-change model as an example. Psychol Behav Sci Int J. 2017;2(2):555-85.

106. Rogers EM. Diffusion of preventive innovations. Addict Behav. 2002;27(6):989-93.

107. Bartholomew LK, Parcel GS, Kok G. Intervention mapping: a process for developing theory- and evidencebased health education programs. Health education \& behavior : the official publication of the Society for Public Health Education. 1998;25(5):545-63.

108. Lorencatto F, West R, Seymour N, Michie S. Developing a method for specifying the components of behavior change interventions in practice: the example of smoking cessation. J Consult Clin Psychol. 2013;81(3):528-44.

109. Bandura A. Social foundations of thought and action. Englewood Cliffs, NJ: Prentice Hall; 1986. 617 p.

110. Prochaska JO, DiClemente CC. Stages and processes of self-change of smoking: toward an integrative model of change. J Consult Clin Psychol. 1983;51(3):390-5.

111. Janz NK, Becker MH. The Health Belief Model: a decade later. Health Educ Q. 1984;11(1):1-47.

112. Ajzen I. The theory of planned behavior. Organizational Behavior and Human Decision Processes. 1991;50(2):179-211.

113. Fishbein M, Ajzen I. Belief, attitude, intention, and behavior: An introduction to theory and research: Philosophy and Rhetoric; 1977.

114. de Ruijter D, Smit E, de Vries H, Goossens L, Hoving C. Understanding Dutch practice nurses' adherence to evidence-based smoking cessation guidelines and their needs for web-based adherence support: results from semistructured interviews. BMJ open. 2017;7(3):e014154.

115. Bolman $\mathrm{C}$, de Vries $\mathrm{H}$, Mesters I. Factors determining cardiac nurses' intentions to continue using a smoking cessation protocol. Heart \& Lung. 2002;31(1):15-24.

116. Hoving $\mathrm{C}$, Mudde AN, de Vries $\mathrm{H}$. Intention to adopt a smoking cessation expert system within a self-selected sample of Dutch general practitioners. European journal of cancer prevention. 2006;15(1):82-6.

117. Berndt NC, Bolman C, de Vries H, Segaar D, van Boven I, Lechner L. Smoking cessation treatment practices: recommendations for improved adoption on cardiology wards. J Cardiovasc Nurs. 2013;28(1):35-47.

118. Verbiest ME, Crone MR, Scharloo M, Chavannes NH, van der Meer V, Kaptein AA, et al. One-hour training for general practitioners in reducing the implementation gap of smoking cessation care: a cluster-randomized controlled trial. Nicotine \& tobacco research : official journal of the Society for Research on Nicotine and Tobacco. 2014;16(1):1-10. 


\section{APPENDICES}

119. Stead M, Angus K, Holme I, Cohen D, Tait G, Team PER. Factors influencing European GPs' engagement in smoking cessation: a multi-country literature review. The British journal of general practice : the journal of the Royal College of General Practitioners. 2009;59(566):682-90.

120. Brotons $C$, Bjorkelund C, Bulc M, Ciurana R, Godycki-Cwirko M, Jurgova E, et al. Prevention and health promotion in clinical practice: the views of general practitioners in Europe. Prev Med. 2005;40(5):595-601.

121. Thompson-Leduc $P$, Turcotte $S$, Labrecque M, Legare F. Prevalence of clinically significant decisional conflict: an analysis of five studies on decision-making in primary care. BMJ Open. 2016;6(6):e011490.

122. Janis IL, Mann L. Decision making: A psychological analysis of conflict, choice, and commitment: Free Press; 1977.

123. Charles C, Gafni A, Whelan T. Shared decision-making in the medical encounter: what does it mean?(or it takes at least two to tango). Social science \& medicine. 1997;44(5):681-92.

124. Stewart M. Studies of health outcomes and patient-centred communication. Thousand Oaks: Sage Publications; 1995.

125. Elwyn G, Edwards A, Kinnersley P. Shared decision-making in primary care: the neglected second half of the consultation. The British journal of general practice : the journal of the Royal College of General Practitioners. 1999;49(443):477-82.

126. Gafni A, Charles $C$, Whelan T. The physician-patient encounter: The physician as a perfect agent for the patient versus the informed treatment decision-making model. Social Science \& Medicine. 1998;47(3):347-54.

127. Stacey D, Legare F, Lewis K, Barry MJ, Bennett CL, Eden KB, et al. Decision aids for people facing health treatment or screening decisions. Cochrane Database Syst Rev. 2017;4(4):CD001431.

128. Montori VM, LeBlanc A, Buchholz A, Stilwell DL, Tsapas A. Basing information on comprehensive, critically appraised, and up-to-date syntheses of the scientific evidence: a quality dimension of the International Patient Decision Aid Standards. BMC Med Inform Decis Mak. 2013;13 Suppl 2(2):S5.

129. Fagerlin A, Pignone M, Abhyankar P, Col N, Feldman-Stewart D, Gavaruzzi T, et al. Clarifying values: an updated review. BMC Med Inform Decis Mak. 2013;13 Suppl 2(2):S8.

130. Elwyn G, O'Connor A, Stacey D, Volk R, Edwards AG, Coulter A, et al. International Patient Decision Aids Standards (IPDAS) Collaboration. Developing a quality criteria framework for patient decision aid: online international Delphi consensus process. British Medical Journal. 2006;333(7565):417-9.

131. Geraghty AWA, Torres LD, Leykin Y, Pérez-Stable EJ, Muñoz RF. Understanding attrition from international internet health interventions: a step towards global eHealth. Health Promotion International. 2012;28(3):442-52.

132. Smit ES, Hoving C, Cox VC, de Vries H. Influence of recruitment strategy on the reach and effect of a webbased multiple tailored smoking cessation intervention among Dutch adult smokers. Health Educ Res. 2012;27(2):191-9.

133. Stanczyk N, De Vries H, Candel M, Muris J, Bolman C. Effectiveness of video-versus text-based computertailored smoking cessation interventions among smokers after one year. Preventive medicine. 2016;82:42-50.

134. Vluggen S, Candel M, Hoving C, Schaper NC, de Vries H. A Web-Based Computer-Tailored Program to Improve Treatment Adherence in Patients With Type 2 Diabetes: Randomized Controlled Trial. Journal of medical Internet research. 2021;23(2):e18524.

135. Gakidou E, Afshin A, Abajobir AA, Abate $\mathrm{KH}$, Abbafati C, Abbas KM, et al. Global, regional, and national comparative risk assessment of 84 behavioural, environmental and occupational, and metabolic risks or clusters of risks, 1990-2016: a systematic analysis for the Global Burden of Disease Study 2016. The Lancet. 2017;390(10100):1345-422.

136. Bommelé J, Hipple Walters B, Willemsen M. Smoking in the Netherlands: Key statistics for 2018. Trimbos; 2020.

137. Hughes JR, Keely J, Naud S. Shape of the relapse curve and long-term abstinence among untreated smokers. Addiction. 2004;99(1):29-38.

138. Chaiton M, Diemert L, Cohen JE, Bondy SJ, Selby P, Philipneri A, et al. Estimating the number of quit attempts it takes to quit smoking successfully in a longitudinal cohort of smokers. BMJ Open. 2016;6(6):e011045.

139. van Hassel D, Batenburg R, van der Velden L. Praktijkondersteuners (POH's) in beeld: Aantallen, kenmerken en geografische spreiding in Nederland. Utrecht: NIVEL; 2016. Report No.: 9461224087.

140. Kotz D, Willemsen MC, Brown J, West R. Light smokers are less likely to receive advice to quit from their GP than moderate-to-heavy smokers: a comparison of national survey data from the Netherlands and England. The European journal of general practice. 2013;19(2):99-105.

141. Vogt F, Hall S, Marteau TM. General practitioners' and family physicians' negative beliefs and attitudes towards discussing smoking cessation with patients: a systematic review. Addiction. 2005;100(10):1423-31. 
142. Lindson N, Klemperer E, Hong B, Ordonez-Mena JM, Aveyard P. Smoking reduction interventions for smoking cessation. Cochrane Database Syst Rev. 2019;9(9):CD013183.

143. Abidi L, Oenema A, Nilsen P, Anderson P, van de Mheen D. Strategies to Overcome Barriers to Implementation of Alcohol Screening and Brief Intervention in General Practice: a Delphi Study Among Healthcare Professionals and Addiction Prevention Experts. Prevention Science. 2016;17(6):689-99.

144. Elfeddali I, Bolman C, Mesters I, Wiers RW, de Vries H. Factors underlying smoking relapse prevention: results of an international Delphi study. Health Education Research. 2010;25(6):1008-20.

145. Glasgow RE, Lichtenstein E, Marcus AC. Why Don't We See More Translation of Health Promotion Research to Practice? Rethinking the Efficacy-to-Effectiveness Transition. American Journal of Public Health. 2003;93(8):1261-7.

146. Adler M, Ziglio E. Gazing into the oracle: The Delphi method and its application to social policy and public health: Jessica Kingsley Publishers; 1996.

147. Taylor E. We Agree, Don't We? The Delphi Method for Health Environments Research. HERD: Health Environments Research \& Design Journal. 2020;13(1):11-23.

148. Gale NK, Heath G, Cameron E, Rashid S, Redwood S. Using the framework method for the analysis of qualitative data in multi-disciplinary health research. BMC medical research methodology. 2013;13(1):117.

149. Landis JR, Koch GG. The measurement of observer agreement for categorical data. biometrics. 1977:159-74.

150. Partnership Stop met Roken. Kwaliteitsregister Stoppen met Roken 2020 [Available from: https://www.kabiz. $\mathrm{nl} /$ beroepen/beroep.aspx?onderwerp=stoppen-met-roken-coach.

151. Linstone HA. The delphi technique. Environmental impact assessment, technology assessment, and risk analysis: Springer; 1985. p. 621-49.

152. Sanders D, Peveler R, Mant D, Fowler G. Predictors of successful smoking cessation following advice from nurses in general practice. Addiction. 1993;88(12):1699-705.

153. Richmond RL, Kehoe LA, Webster IW. Multivariate models for predicting abstention following intervention to stop smoking by general practitioners. Addiction. 1993;88(8):1127-35.

154. Butler CC, Rollnick S, Cohen D, Bachmann M, Russell I, Stott N. Motivational consulting versus brief advice for smokers in general practice: a randomized trial. The British Journal of General Practice. 1999;49(445):611.

155. Soria R, Legido A, Escolano C, Yeste AL, Montoya J. A randomised controlled trial of motivational interviewing for smoking cessation. British journal of general practice. 2006;56(531):768-74.

156. Papadakis S, McDonald P, Mullen K-A, Reid R, Skulsky K, Pipe A. Strategies to increase the delivery of smoking cessation treatments in primary care settings: a systematic review and meta-analysis. Preventive medicine. 2010;51(3-4):199-213.

157. Anderson $P$, Jané-Llopis $E$. How can we increase the involvement of primary health care in the treatment of tobacco dependence? A meta-analysis. Addiction. 2004;99(3):299-312.

158. Okoli CT, Greaves L, Bottorff JL, Marcellus LM. Health care providers' engagement in smoking cessation with pregnant smokers. Journal of Obstetric, Gynecologic \& Neonatal Nursing. 2010;39(1):64-77.

159. Castles A, Adams EK, Melvin CL, Kelsch C, Boulton ML. Effects of smoking during pregnancy: five metaanalyses. American journal of preventive medicine. 1999;16(3):208-15.

160. Hartmann-Boyce J, McRobbie H, Bullen C, Begh R, Stead L, Hajek P. Can electronic cigarettes help people stop smoking, and are they safe to use for this purpose. Cochrane Database Syst Rev. 2016;9.

161. Diemert L, Bayoumy D, Pelletier H, Schwartz R, O'Connor S. E-Cigarette Use for Smoking Cessation. 2019.

162. Hartmann-Boyce J, McRobbie H, Bullen C, Begh R, Stead LF, Hajek P. Electronic cigarettes for smoking cessation. Cochrane Database of Systematic Reviews. 2016(9).

163. Perrine CG, Pickens CM, Boehmer TK, King BA, Jones CM, DeSisto CL, et al. Characteristics of a multistate outbreak of lung injury associated with e-cigarette use, or vaping-United States, 2019. Morbidity and Mortality Weekly Report. 2019;68(39):860.

164. Warner KE. How to Think-Not Feel-about Tobacco Harm Reduction. Nicotine \& Tobacco Research. 2018;21(10):1299-309.

165. Hall SM, Humfleet GL, Muñoz RF, Reus VI, Robbins JA, Prochaska JJ. Extended treatment of older cigarette smokers. Addiction. 2009;104(6):1043-52.

166. Luijks HD, Loeffen MJ, Lagro-Janssen AL, Van Weel C, Lucassen PL, Schermer TR. GPs' considerations in multimorbidity management: a qualitative study. British Journal of General Practice. 2012;62(600):e503-e10.

167. Bakker EC, Nijkamp MD, Sloot C, Berndt NC, Bolman CA. Intention to abstain from smoking among cardiac rehabilitation patients: the role of attitude, self-efficacy, and craving. Journal of Cardiovascular Nursing. 2015;30(2):172-9. 


\section{APPENDICES}

168. Berndt N, Bolman C, Mudde A, Verheugt F, de Vries H, Lechner L. Risk groups and predictors of short-term abstinence from smoking in patients with coronary heart disease. heart \& lung. 2012;41(4):332-43.

169. Jiménez-Ruiz CA, Masa F, Miravitlles M, Gabriel R, Viejo JL, Villasante C, et al. Smoking characteristics: differences in attitudes and dependence between healthy smokers and smokers with COPD. Chest. 2001;119(5):1365-70.

170. Kotsoni C, Antonakis N, Markaki A, Lionis C. Do patients with chronic obstructive pulmonary disease receive smoking cessation advice and interventions in rural Crete? Report from a medical audit study. Australian Journal of Rural Health. 2008;16(6):385-6.

171. Erku DA, Gartner CE, Morphett K, Steadman KJ. Beliefs and Self-reported Practices of Health Care Professionals Regarding Electronic Nicotine Delivery Systems: A Mixed-Methods Systematic Review and Synthesis. Nicotine \& Tobacco Research. 2019;22(5):619-29.

172. Chavannes H, Kaper J, Frijling D, Van der Laan R, Jansen M, Guerrouj S, et al. NHG-Standaard Stoppen met roken. NHG-Standaarden 2009: Springer; 2009. p. 1434-49.

173. Swanborn PG. Methoden van sociaal-wetenschappelijk onderzoek: Boom Koninklijke Uitgevers; 1987.

174. Freund T, Everett C, Griffiths P, Hudon C, Naccarella L, Laurant M. Skill mix, roles and remuneration in the primary care workforce: who are the healthcare professionals in the primary care teams across the world? International journal of nursing studies. 2015;52(3):727-43.

175. Peacock A, Leung J, Larney S, Colledge S, Hickman M, Rehm J, et al. Global statistics on alcohol, tobacco and illicit drug use: 2017 status report. Addiction. 2018;113(10):1905-26.

176. CBS. Doodsoorzakenstatistiek. Den Haag: Centraal Bureau voor de Statistiek (CBS). 2018.

177. Fiore MC, Bailey WC, Cohen SJ, Dorfman SF, Goldstein MG, Gritz ER, et al. Treating tobacco use and dependence: clinical practice guideline. Rockville, MD: US Department of Health and Human Services. 2000:00 0032.

178. Te Poel F, Bolman C, Reubsaet A, de Vries H. Efficacy of a single computer-tailored e-mail for smoking cessation: results after 6 months. Health Educ Res. 2009;24(6):930-40.

179. Berndt N, Bolman C, Lechner L, Max W, Mudde A, de Vries H, et al. Economic evaluation of a telephone-and face-to-face-delivered counseling intervention for smoking cessation in patients with coronary heart disease. The European Journal of Health Economics. 2016;17(3):269-85.

180. Boerdam A, Bevolkingstrends KK. Astma en COPD in beeld. Den Haag: Centraal Bureau voor de Statistiek. 2016.

181. Guassora AD, Gannik D. Developing and maintaining patients' trust during general practice consultations: the case of smoking cessation advice. Patient Education and Counseling. 2010;78(1):46-52.

182. Dierick-van Daele AT, Metsemakers JF, Derckx EW, Spreeuwenberg C, Vrijhoef HJ. Nurse practitioners substituting for general practitioners: randomized controlled trial. J Adv Nurs. 2009;65(2):391-401.

183. de Ruijter D, Smit ES, de Vries H, Hoving C. Web-based computer-tailoring for practice nurses aimed to improve smoking cessation guideline adherence: A study protocol for a randomized controlled effectiveness trial. Contemporary clinical trials. 2016;48:125-32.

184. Cabana MD, Rand CS, Powe NR, Wu AW, Wilson MH, Abboud P-AC, et al. Why don't physicians follow clinical practice guidelines?: A framework for improvement. Jama. 1999;282(15):1458-65.

185. Abrahamson KA, Fox RL, Doebbeling BN. Facilitators and barriers to clinical practice guideline use among nurses. AJN The American Journal of Nursing. 2012;112(7):26-35.

186. Ministerie van Algemene Zaken. Taalniveau B1 2019 [

187. White AR, Rampes H, Liu JP, Stead LF, Campbell J. Acupuncture and related interventions for smoking cessation. Cochrane Database of Systematic Reviews. 2014(1).

188. Kerr CM, Lowe PB, Spielholz NI. Low level laser for the stimulation of acupoints for smoking cessation: a double blind, placebo controlled randomised trial and semi structured interviews. JOURNAL OF CHINESE MEDICINE-HOVE-. 2008;86:46.

189. Bier ID, Wilson J, Studt P, Shakleton M. Auricular acupuncture, education, and smoking cessation: a randomized, sham-controlled trial. American Journal of Public Health. 2002;92(10):1642-7.

190. Carmody TP, Duncan C, Simon JA, Solkowitz S, Huggins J, Lee S, et al. Hypnosis for smoking cessation: a randomized trial. Nicotine \& Tobacco Research. 2008;10(5):811-8.

191. Kalkhoran S, Glantz SA. E-cigarettes and smoking cessation in real-world and clinical settings: a systematic review and meta-analysis. The Lancet Respiratory Medicine. 2016;4(2):116-28.

192. Cheung KL, de Ruijter D, Hiligsmann M, Elfeddali I, Hoving C, Evers SM, et al. Exploring consensus on how to measure smoking cessation. A Delphi study. BMC Public Health. 2017;17(1):890. 
193. Mudde A, Willemsen M, Kremers S, De Vries H. Meetinstrumenten voor onderzoek naar roken en stoppen met roken.(Measurements from studies about smoking and smoking cessation). Den Haag, the Netherlands: Stivoro. 2006.

194. Aguinis H, Pierce CA, Quigley BM. Conditions Under Which a Bogus Pipeline Procedure Enhances the Validity of Self-Reported Cigarette Smoking: A Meta-Analytic Review. Journal of Applied Social Psychology. 1993;23(5):352-73.

195. Adams J, Parkinson L, Sanson-Fisher RW, Walsh RA. Enhancing self-report of adolescent smoking: the effects of bogus pipeline and anonymity. Addict Behav. 2008;33(10):1291-6.

196. Glasgow RE, Mullooly JP, Vogt TM, Stevens VJ, Lichtenstein E, Hollis JF, et al. Biochemical validation of smoking status: pros, cons, and data from four low-intensity intervention trials. Addict Behav. 1993;18(5):511-27.

197. Patrick DL, Cheadle A, Thompson DC, Diehr P, Koepsell T, Kinne S. The validity of self-reported smoking: a review and meta-analysis. Am J Public Health. 1994;84(7):1086-93.

198. Velicer WF, Prochaska JO. A comparison of four self-report smoking cessation outcome measures. Addict Behav. 2004;29(1):51-60.

199. Janssen MF, Pickard AS, Golicki D, Gudex C, Niewada M, Scalone L, et al. Measurement properties of the EQ-5D-5L compared to the EQ-5D-3L across eight patient groups: a multi-country study. Quality of Life Research. 2013;22(7):1717-27.

200. Al-Janabi H, N Flynn T, Coast J. Development of a self-report measure of capability wellbeing for adults: the ICECAP-A. Quality of Life Research. 2012;21(1):167-76.

201. Bouwmans C, Krol M, Severens H, Koopmanschap M, Brouwer W, Hakkaart-van Roijen L. The iMTA productivity cost questionnaire: a standardized instrument for measuring and valuing health-related productivity losses. Value in health. 2015;18(6):753-8.

202. Tan SS, Bouwmans-Frijters CA, Hakkaart-van Roijen L. Handleiding voor kostenonderzoek: methoden en referentieprijzen voor economische evaluaties in de gezondheidszorg. Tijdschrift voor gezondheidswetenschappen. 2012;90(6):367-72.

203. Hakkaart-van Roijen L, Van der Linden N, Bouwmans C, Kanters T, Tan SS. Kostenhandleiding. Methodologie van kostenonderzoek en referentieprijzen voor economische evaluaties in de gezondheidszorg In opdracht van Zorginstituut Nederland Geactualiseerde versie. 2015.

204. O'Connor AM. User manual-decisional conflict scale. Ottawa: Ottawa Hospital Research Institute [Internet]. 2010. Available from: http://decisionaid.ohri.ca/docs/develop/User_Manuals/UM_Decisional_Conflict.pdf.

205. O'Connor AM. Validation of a decisional conflict scale. Medical decision making. 1995;15(1):25-30.

206. Rogers EM. Diffusion of innovations. New York: Simon and Schuster; 2010.

207. Bolman C, de Vries H, van Breukelen G. A Minimal-Contact Intervention for Cardiac Inpatients: Long-Term Effects on Smoking Cessation. Preventive Medicine. 2002;35(2):181-92.

208. Fagerström K. Determinants of tobacco use and renaming the FTND to the Fagerström Test for Cigarette Dependence. Nicotine \& Tobacco Research. 2011;14(1):75-8.

209. Heatherton TF, Kozlowski LT, Frecker RC, Fagerstrom KO. The Fagerstrom Test for Nicotine Dependence: a revision of the Fagerstrom Tolerance Questionnaire. British journal of addiction. 1991;86(9):1119-27.

210. Bouwmans C. Manual iMTA Medical Consumption Questionnaire (iMCQ). 2013a. 2018.

211. Chew LD, Bradley KA, Boyko EJ. Brief questions to identify patients with inadequate health literacy. health. 2004;11:12.

212. Faul F, Erdfelder E, Lang A-G, Buchner A. G* Power 3: A flexible statistical power analysis program for the social, behavioral, and biomedical sciences. Behavior research methods. 2007;39(2):175-91.

213. Buuren Sv, Groothuis-Oudshoorn K. mice: Multivariate imputation by chained equations in R. Journal of statistical software. 2010:1-68.

214. Blankers $M$, Smit ES, van der Pol $P$, de Vries $H$, Hoving $C$, van Laar $M$. The missing= smoking assumption: a fallacy in internet-based smoking cessation trials? Nicotine \& Tobacco Research. 2016;18(1):25-33.

215. West R, Hajek P, Stead L, Stapleton J. Outcome criteria in smoking cessation trials: proposal for a common standard. Addiction. 2005;100(3):299-303.

216. Bundy C. Changing behaviour: using motivational interviewing techniques. J R Soc Med. 2004;97 Suppl 44(Suppl 44):43-7.

217. Shahab L, McEwen A. Online support for smoking cessation: a systematic review of the literature. Addiction. 2009;104(11):1792-804.

218. Webb TL. Commentary on Shahab \& McEwen (2009): Understanding and preventing attrition in online smoking cessation interventions: a self-regulatory perspective. Addiction. 2009;104(11):1805-6. 


\section{APPENDICES}

219. Eysenbach G. The law of attrition. J Med Internet Res. 2005;7(1):e11.

220. Wampold BE. How important are the common factors in psychotherapy? An update. World Psychiatry. 2015;14(3):270-7.

221. Kok L, Berden C, Koopmans C. Kosten van Roken. SEO economisch Onderzoek. 2016.

222. Ellerbeck EF, Ahluwalia JS, Jolicoeur DG, Gladden J, Mosier MC. Direct observation of smoking cessation activities in primary care practice. The Journal of family practice. 2001;50(8):688-93.

223. McCaughan D, Thompson C, Cullum N, Sheldon T, Raynor P. Nurse practitioner and practice nurses' use of research information in clinical decision making: findings from an exploratory study. Family Practice. 2005;22(5):490-7.

224. Springvloet L, Bommele J, Willemsen M, Van Laar M. Kerncijfers roken 2017. Utrecht: Trimbos-instituut; 2018.

225. Zijlstra DN, Hoving C, Bolman C, Muris JWM, De Vries H. Do professional perspectives on evidence-based smoking cessation methods align? A Delphi study among researchers and healthcare professionals. Health Educ Res. 2021.

226. Cheung KL, Wijnen BF, Hiligsmann M, Coyle K, Coyle D, Pokhrel S, et al. Is it cost-effective to provide internetbased interventions to complement the current provision of smoking cessation services in the Netherlands? An analysis based on the EQUIPTMOD. Addiction. 2018;113:87-95.

227. Cahill K, Lancaster T, Perera R. Pharmacological interventions for smoking cessation: an overview of reviews. Cochrane Database of Systematic Reviews. 2011(9).

228. Zijlstra DN, Muris JWM, Bolman C, Elling JM, Knapen V, de Vries H. A referral aid for smoking cessation interventions in primary care: study protocol for a randomized controlled trial. Prim Health Care Res Dev. 2021;22:e22.

229. Figueras N, North B, Takala S, Van Avermaet P, Verhelst N. Relating language examinations to the common European framework of reference for languages: learning, teaching, assessment (CEFR): a manual. 2009.

230. Hulstijn JH, Schoonen R, De Jong NH, Steinel MP, Florijn A. Linguistic competences of learners of Dutch as a second language at the B1 and B2 levels of speaking proficiency of the Common European Framework of Reference for Languages (CEFR). Language Testing. 2012;29(2):203-21.

231. Schulz KF, Altman DG, Moher D. CONSORT 2010 Statement: updated guidelines for reporting parallel group randomised trials. BMJ. 2010;340:c332.

232. Jakobsen JC, Gluud C, Wetterslev J, Winkel P. When and how should multiple imputation be used for handling missing data in randomised clinical trials - a practical guide with flowcharts. BMC Medical Research Methodology. 2017;17(1):162.

233. Page MJ, French SD, McKenzie JE, O'Connor DA, Green SE. Recruitment difficulties in a primary care cluster randomised trial: investigating factors contributing to general practitioners' recruitment of patients. BMC Medical Research Methodology. 2011;11(1):1-9.

234. Pringle $M$, Churchill R. Randomised controlled trials in general practice. British Medical Journal Publishing Group; 1995.

235. Watson JM, Torgerson DJ. Increasing recruitment to randomised trials: a review of randomised controlled trials. BMC medical research methodology. 2006;6(1):1-9.

236. Newington $L$, Metcalfe A. Factors influencing recruitment to research: qualitative study of the experiences and perceptions of research teams. BMC medical research methodology. 2014;14(1):1-11.

237. Vluggen S, Hoving C, Vonken L, Schaper NC, de Vries H. Exploring factors influencing recruitment results of nurses recruiting diabetes patients for a randomized controlled trial. Clinical Trials. 2020;17(4):448-58.

238. O'Connor AM. Validation of a decisional conflict scale. Medical decision making. 1995;15(1):25-30.

239. Gultzow T, Smit ES, Hudales R, Dirksen CD, Hoving C. Informed decision making on the uptake of evidencebased smoking cessation assistance: A needs assessment among end users and experts to inform decision aid development. medRxiv. 2021:2021.04.09.21255012.

240. Grol R, Wensing M, Eccles M. Improving patient care. The implementation of change in clinical practice Edinburgh, London, New York. Oxford, Philadelphia, St. Louis, Sydney, Toronto: Elsevier; 2005.

241. Vernooij-Dassen M, Moniz-Cook E. Raising the standard of applied dementia care research: addressing the implementation error. Taylor \& Francis; 2014.

242. Orleans CT, Woolf SH, Rothemich SF, Marks JS, Isham GJ. The top priority: building a better system for tobacco-cessation counseling. Am J Prev Med. 2006;31(1):103-6.

243. Newhall K, Burnette M, Brooke BS, Schanzer A, Tan T, Flocke S, et al. Smoking cessation counseling in vascular surgical practice using the results of interviews and focus groups in the Vascular Surgeon offer and report smoking cessation pilot trial. Journal of vascular surgery. 2016;63(4):1011-7. e2. 
244. Nickels AS, Warner DO, Jenkins SM, Tilburt J, Hays JT. Beliefs, practices, and self-efficacy of US physicians regarding smoking cessation and electronic cigarettes: a national survey. Nicotine \& Tobacco Research. 2017;19(2):197-207.

245. Meijer E, Van der Kleij R, Chavannes NH. Facilitating smoking cessation in patients who smoke: a largescale cross-sectional comparison of fourteen groups of healthcare providers. BMC health services research. 2019;19(1):750.

246. de Vries $\mathrm{H}$, Vahl J, Muris J, Evers S, van der Horst $\mathrm{H}$, Cheung KL. Effects of the reform of the Dutch healthcare into managed competition: Results of a Delphi study among experts. Health Policy. 2021;125(1):27-33.

247. Schneider F, Schulz DN, Pouwels LHL, de Vries H, van Osch LADM. The use of a proactive dissemination strategy to optimize reach of an internet-delivered computer tailored lifestyle intervention. BMC Public Health. 2013;13(1):721.

248. Schneider $F$, van Osch $L$, de Vries $H$, editors. Optimising online lifestyle programs by increasing user involvement in the development process: An application of different strategies. PSYCHOLOGY \& HEALTH; 2010: TAYLOR \& FRANCIS LTD 4 PARK SQUARE, MILTON PARK, ABINGDON OX14 4RN, OXON ....

249. World Health Organization. WHO global report on mortality attributable to tobacco. 2012.

250. Hakkennes S, Dodd K. Guideline implementation in allied health professions: a systematic review of the literature. Qual Saf Health Care. 2008;17(4):296-300.

251. Saillour-Glenisson F, Michel P. Individual and collective facilitators of and barriers to the use of clinical practice guidelines by physicians: a literature review. Revue d'epidemiologie et de sante publique. 2003;51(1 Pt 1):65-80.

252. Verbiest ME, Presseau J, Chavannes NH, Scharloo M, Kaptein AA, Assendelft WJ, et al. Use of action planning to increase provision of smoking cessation care by general practitioners: role of plan specificity and enactment. Implementation Science. 2014;9(1):180.

253. Zijlstra D, Bolman C, Muris J, de Vries H. What went wrong? A randomized controlled trial of a process and effect evaluation of a referral aid for smoking cessation counseling in primary care. Submitted for publication.

254. McEwen A, West R. Smoking cessation activities by general practitioners and practice nurses. Tob Control. 2001;10(1):27-32.

255. Bruce N, Burnett S. Prevention of lifestyle-related disease: general practitioners' views about their role, effectiveness and resources. Fam Pract. 1991;8(4):373-7.

256. Park ER, DePue JD, Goldstein MG, Niaura R, Harlow LL, Willey C, et al. Assessing the transtheoretical model of change constructs for physicians counseling smokers. Ann Behav Med. 2003;25(2):120-6.

257. Heckman CJ, Egleston BL, Hofmann MT. Efficacy of motivational interviewing for smoking cessation: a systematic review and meta-analysis. Tob Control. 2010;19(5):410-6.

258. Hall S, Marteau TM. Practice nurses' self-reported opportunistic smoking cessation advice in three contexts. Nicotine \& tobacco research : official journal of the Society for Research on Nicotine and Tobacco. 2007;9(9):941-5.

259. World Health Organization. Global status report on noncommunicable diseases 2014: "Attaining the nine global noncommunicable diseases targets; a shared responsibility”. Geneva; 2014.

260. Forouzanfar MH, Afshin A, Alexander LT, Anderson HR, Bhutta ZA, Biryukov S, et al. Global, regional, and national comparative risk assessment of 79 behavioural, environmental and occupational, and metabolic risks or clusters of risks, 1990-2015: a systematic analysis for the Global Burden of Disease Study 2015. The Lancet. 2016;388(10053):1659-724.

261. Tamakoshi A, Tamakoshi K, Lin Y, Yagyu K, Kikuchi S, Group JS. Healthy lifestyle and preventable death: findings from the Japan Collaborative Cohort (JACC) Study. Prev Med. 2009;48(5):486-92.

262. World Health Organization. WHO | Risk factors. 2018.

263. Wu Y, Zhai L, Zhang D. Sleep duration and obesity among adults: a meta-analysis of prospective studies. Sleep medicine. 2014;15(12):1456-62.

264. Cahill K, Stevens S, Perera R, Lancaster T. Pharmacological interventions for smoking cessation: an overview and network meta-analysis. Cochrane Database Syst Rev. 2013(5):CD009329.

265. Stead LF, Hartmann-Boyce J, Perera R, Lancaster T. Telephone counselling for smoking cessation. Cochrane Database Syst Rev. 2013(8):CD002850.

266. Llewellyn-Thomas HA, Crump RT. Decision support for patients: values clarification and preference elicitation. Med Care Res Rev. 2013;70(1 Suppl):50S-79S.

267. Abhyankar P, Volk RJ, Blumenthal-Barby J, Bravo P, Buchholz A, Ozanne E, et al. Balancing the presentation of information and options in patient decision aids: an updated review. BMC Med Inform Decis Mak. 2013;13 Suppl 2(2):S6. 


\section{APPENDICES}

268. Bekker H, Thornton JG, Airey CM, Connelly JB, Hewison J, Robinson MB, et al. Informed decision making: an annotated bibliography and systematic review. Health technology assessment (Winchester, England). 1999;3(1):1-156.

269. Feldman-Stewart D, Tong C, Siemens R, Alibhai S, Pickles T, Robinson J, et al. The impact of explicit values clarification exercises in a patient decision aid emerges after the decision is actually made: evidence from a randomized controlled trial. Medical decision making : an international journal of the Society for Medical Decision Making. 2012;32(4):616-26.

270. Moyo F, Archibald E, Slyer JT. Effectiveness of decision aids for smoking cessation in adults: a quantitative systematic review. JBI database of systematic reviews and implementation reports. 2018;16(9):1791-822.

271. Witteman HO, Gavaruzzi T, Scherer LD, Pieterse AH, Fuhrel-Forbis A, Chipenda Dansokho S, et al. Effects of Design Features of Explicit Values Clarification Methods: A Systematic Review. Medical decision making : an international journal of the Society for Medical Decision Making. 2016;36(6):760-76.

272. Durand MA, Stiel M, Boivin J, Elwyn G. Where is the theory? Evaluating the theoretical frameworks described in decision support technologies. Patient Educ Couns. 2008;71(1):125-35.

273. Trenaman L, Bryan S, Bansback N. The cost-effectiveness of patient decision aids: A systematic review. Healthcare. 2014;2(4):251-7.

274. Arksey H, O'Malley L. Scoping studies: towards a methodological framework. International Journal of Social Research Methodology. 2005;8(1):19-32.

275. Peters MDJ, Godfrey C, McInerney P, Baldini Soares C, Khalil H, Parker D. Chapter 11: scoping reviews. In: Aromataris E, Munn Z, Aromataris E, Munn Z, editors. Joanna Briggs Institute Reviewer's Manual2017.

276. Tricco AC, Lillie E, Zarin W, O’Brien KK, Colquhoun H, Levac D, et al. PRISMA Extension for Scoping Reviews (PRISMA-ScR): Checklist and Explanation. Annals of internal medicine. 2018;169(7):467-73.

277. Gültzow T, Zijlstra D, Bolman C, Vries dH, Dirksen C, Jongen S, et al. Study Design. 2018.

278. Ottawa Hospital Research Institute. Decision Aid Library Inventory - Patient Decision Aids n.d. [Available from: https://decisionaid.ohri.ca/cochinvent.php.

279. Volk RJ, Llewellyn-Thomas H, Stacey D, Elwyn G. Ten years of the International Patient Decision Aid Standards Collaboration: evolution of the core dimensions for assessing the quality of patient decision aids. BMC Med Inform Decis Mak. 2013;13 Suppl 2(2):S1.

280. International Patient Decision Aids Standards (IPDAS) Collaboration. International Patient Decision Aids Standards (IPDAS) Collaboration [Available from: http://ipdas.ohri.ca/what.html.

281. Cupples ME, Cole JA, Hart ND, Heron N, McKinley MC, Tully MA. Shared decision-making (SHARE-D) for healthy behaviour change: a feasibility study in general practice. BJGP Open. 2018;2(2):bjgpopen18X101517.

282. Geller KS, Mendoza ID, Timbobolan J, Montjoy HL, Nigg CR. The decisional balance sheet to promote healthy behavior among ethnically diverse older adults. Public health nursing (Boston, Mass). 2012;29(3):241-6.

283. Hirsch O, Keller H, Albohn-Kuhne C, Krones T, Donner-Banzhoff N. Satisfaction of patients and primary care physicians with shared decision making. Evaluation \& the health professions. 2010;33(3):321-42.

284. Hirsch O, Keller H, Albohn-Kuhne C, Krones T, Donner-Banzhoff N. Pitfalls in the statistical examination and interpretation of the correspondence between physician and patient satisfaction ratings and their relevance for shared decision making research. BMC medical research methodology. 2011;11:71.

285. Hirsch O, Keller H, Krones T, Donner-Banzhoff N. Acceptance of shared decision making with reference to an electronic library of decision aids (arriba-lib) and its association to decision making in patients: an evaluation study. Implementation science : IS. 2011;6(1):70.

286. Hirsch $\mathrm{O}$, Keller H, Krones T, Donner-Banzhoff N. Arriba-lib: evaluation of an electronic library of decision aids in primary care physicians. BMC Med Inform Decis Mak. 2012;12:48.

287. Hirsch $\mathrm{O}$, Keller H, Krones T, Donner-Banzhoff N. Arriba-lib: association of an evidence-based electronic library of decision aids with communication and decision-making in patients and primary care physicians. International Journal of Evidence-Based Healthcare. 2012;10(1):68-76.

288. Hirsch O, Szabo E, Keller H, Kramer L, Krones T, Donner-Banzhoff N. arriba-lib: Analyses of user interactions with an electronic library of decision aids on the basis of log data. Informatics for Health and Social Care. 2012;37(4):264-76.

289. Koelewijn-van Loon MS, van Steenkiste B, Ronda G, Wensing M, Stoffers HE, Elwyn G, et al. Improving patient adherence to lifestyle advice (IMPALA): a cluster-randomised controlled trial on the implementation of a nurse-led intervention for cardiovascular risk management in primary care (protocol). BMC health services research. 2008;8(1):9. 
290. Koelewijn-van Loon MS, van der Weijden T, van Steenkiste B, Ronda G, Winkens B, Severens JL, et al. Involving patients in cardiovascular risk management with nurse-led clinics: a cluster randomized controlled trial. CMAJ : Canadian Medical Association journal = journal de l'Association medicale canadienne. 2009;181(12):E267-74.

291. Koelewijn-van Loon MS, van der Weijden T, Ronda G, van Steenkiste B, Winkens B, Elwyn G, et al. Improving lifestyle and risk perception through patient involvement in nurse-led cardiovascular risk management: a cluster-randomized controlled trial in primary care. Prev Med. 2010;50(1-2):35-44.

292. Krones T, Keller H, Sonnichsen A, Sadowski EM, Baum E, Wegscheider K, et al. Absolute cardiovascular disease risk and shared decision making in primary care: a randomized controlled trial. Ann Fam Med. 2008;6(3):218-27.

293. Krones T, Keller H, Becker A, Sonnichsen A, Baum E, Donner-Banzhoff N. The theory of planned behaviour in a randomized trial of a decision aid on cardiovascular risk prevention. Patient Educ Couns. 2010;78(2):169-76.

294. Sheridan SL, Griffith JM, Behrend L, Gizlice Z, Jianwen C, Pignone MP. Effect of adding a values clarification exercise to a decision aid on heart disease prevention: a randomized trial. Medical decision making : an international journal of the Society for Medical Decision Making. 2010;30(4):E28-39.

295. Sheridan SL, Draeger LB, Pignone MP, Keyserling TC, Simpson RJ, Jr., Rimer B, et al. A randomized trial of an intervention to improve use and adherence to effective coronary heart disease prevention strategies. BMC health services research. 2011;11:331.

296. Sheridan SL, Draeger LB, Pignone MP, Sloane PD, Samuel-Hodge C, Finkelstein EA, et al. Designing and implementing a comparative effectiveness study of two strategies for delivering high quality CHD prevention: methods and participant characteristics for the Heart to Health study. Contemporary clinical trials. 2013;36(2):394-405.

297. Sheridan SL, Draeger LB, Pignone MP, Rimer B, Bangdiwala SI, Cai J, et al. The effect of a decision aid intervention on decision making about coronary heart disease risk reduction: secondary analyses of a randomized trial. BMC Med Inform Decis Mak. 2014;14:14.

298. Keyserling TC, Sheridan SL, Draeger LB, Finkelstein EA, Gizlice Z, Kruger E, et al. A comparison of live counseling with a web-based lifestyle and medication intervention to reduce coronary heart disease risk: a randomized clinical trial. JAMA internal medicine. 2014;174(7):1144-57.

299. Tinsel I, Siegel A, Schmoor C, Buchholz A, Niebling W. DECADE-pilot: decision aid, action planning, and followup support for patients to reduce the 10-year risk of cardiovascular diseases-a protocol of a randomized controlled pilot trial. Pilot Feasibility Stud. 2017;3:32.

300. Tinsel I, Siegel A, Schmoor C, Poguntke I, Maun A, Niebling W. Encouraging Self-Management in Cardiovascular Disease Prevention. Dtsch Arztebl Int. 2018;115(27-28):469-76.

301. van Steenkiste B, van der Weijden TM, Stoffers JH, Grol RP. Patients' responsiveness to a decision support tool for primary prevention of cardiovascular diseases in primary care. Patient Educ Couns. 2008;72(1):63-70.

302. BinDhim NF, McGeechan K, Trevena L. Assessing the effect of an interactive decision-aid smartphone smoking cessation application (app) on quit rates: a double-blind automated randomised control trial protocol. BMJ Open. 2014;4(7):e005371.

303. BinDhim NF, McGeechan K, Trevena L. Smartphone Smoking Cessation Application (SSC App) trial: a multicountry double-blind automated randomised controlled trial of a smoking cessation decision-aid 'app'. BMJ Open. 2018;8(1):e017105.

304. Brunette MF, Ferron JC, McHugo GJ, Davis KE, Devitt TS, Wilkness SM, et al. An electronic decision support system to motivate people with severe mental illnesses to quit smoking. Psychiatric services (Washington, DC). 2011;62(4):360-6.

305. Brunette MF, Ferron JC, Drake RE, Devitt TS, Geiger PT, McHugo GJ, et al. Carbon monoxide feedback in a motivational decision support system for nicotine dependence among smokers with severe mental illnesses. Journal of substance abuse treatment. 2013;45(4):319-24.

306. Brunette MF, Gunn W, Alvarez H, Finn PC, Geiger P, Ferron JC, et al. A pre-post pilot study of a brief, webbased intervention to engage disadvantaged smokers into cessation treatment. Addiction science \& clinical practice. 2015;10(1):3.

307. Cupertino AP, Richter K, Cox LS, Garrett S, Ramirez R, Mujica F, et al. Feasibility of a Spanish/English computerized decision aid to facilitate smoking cessation efforts in underserved communities. Journal of health care for the poor and underserved. 2010;21(2):504-17.

308. Ferron JC, Brunette MF, McHugo GJ, Devitt TS, Martin WM, Drake RE. Developing a quit smoking website that is usable by people with severe mental illnesses. Psychiatric rehabilitation journal. 2011;35(2):111-6. 


\section{APPENDICES}

309. Ferron JC, Brunette MF, McGurk SR, Xie H, Frounfelker R, Cook JA, et al. Do Symptoms and Cognitive Problems Affect the Use and Efficacy of a Web-Based Decision Support System for Smokers With Serious Mental Illness? Journal of Dual Diagnosis. 2012;8(4):315-25.

310. Hollen PJ, Tyc VL, Donnangelo SF, Shannon SV, O'Laughlen MC, Hinton I, et al. A substance use decision aid for medically at-risk adolescents: results of a randomized controlled trial for cancer-surviving adolescents. Cancer nursing. 2013;36(5):355-67.

311. Lee JE, Shin DW, Suh B, Chun S, Nam YS, Cho B. Development and application of culturally appropriate decision aids for smoking cessation in Korea: a pragmatic clustered randomization crossover trial. Patient Prefer Adherence. 2016;10:1929-36.

312. McDonnell KK, Bullock LF, Kozower BD, Hollen PJ, Heath J, Rovnyak V. A decision aid to improve smoking abstinence for families facing cancer. Oncology nursing forum. 2014;41(6):649-58.

313. McDonnell KK, Hollen PJ, Heath J, Andrews JO. Recruiting family dyads facing thoracic cancer surgery: Challenges and lessons learned from a smoking cessation intervention. European journal of oncology nursing : the official journal of European Oncology Nursing Society. 2016;20:199-206.

314. Rhee H, Hollen PJ, Belyea MJ, Sutherland MA. Decision-making program for rural adolescents with asthma: a pilot study. J Pediatr Nurs. 2008;23(6):439-50.

315. Warner DO, LeBlanc A, Kadimpati S, Vickers KS, Shi Y, Montori VM. Decision Aid for Cigarette Smokers Scheduled for Elective Surgery. Anesthesiology. 2015;123(1):18-28.

316. van Steenkiste B, van der Weijden T, Stoffers HE, Kester AD, Timmermans DR, Grol R. Improving cardiovascular risk management: a randomized, controlled trial on the effect of a decision support tool for patients and physicians. European journal of cardiovascular prevention and rehabilitation : official journal of the European Society of Cardiology, Working Groups on Epidemiology \& Prevention and Cardiac Rehabilitation and Exercise Physiology. 2007;14(1):44-50.

317. Healthwise S. Quitting Smoking: Should I Use Medicine? | Kaiser Permanente.

318. Healthwise S. Obesity: Should I Use a Diet Plan to Lose Weight?

319. Healthwise S. Weight Management: Should I Use Over-the-Counter Diet Aids? | Kaiser Permanente.

320. Healthwise S. Sleep Apnea: Should I Have a Sleep Study?

321. Kok G, Gottlieb NH, Peters GJ, Mullen PD, Parcel GS, Ruiter RA, et al. A taxonomy of behaviour change methods: an Intervention Mapping approach. null. 2016;10(3):297-312.

322. Witteman HO, Julien AS, Ndjaboue R, Exe NL, Kahn VC, Angie Fagerlin A, et al. What Helps People Make Values-Congruent Medical Decisions? Eleven Strategies Tested across 6 Studies. Medical decision making : an international journal of the Society for Medical Decision Making. 2020;40(3):266-78.

323. Ryan RM, Deci EL. Self-determination theory and the facilitation of intrinsic motivation, social development, and well-being. Am Psychol. 2000;55(1):68-78.

324. Peters G-JY, Crutzen R. Pragmatic nihilism: how a Theory of Nothing can help health psychology progress. Health Psychology Review. 2017;11(2):103-21.

325. Ryan RM, Patrick H, Deci EL, Williams GC. Facilitating health behaviour change and its maintenance: Interventions based on self-determination theory. The European Health Psychologist. 2008;10(1):2-5.

326. Su Y-L, Reeve J. A Meta-analysis of the Effectiveness of Intervention Programs Designed to Support Autonomy. Educational Psychology Review. 2010;23(1):159-88.

327. Mariolis P, Rock V, Asman K, Merritt R, Malarcher A, Husten C, et al. Tobacco use among adults-United States, 2005. Oncology Times. 2006;28(22):42-7.

328. Zwar NA, Richmond RL. Role of the general practitioner in smoking cessation. Drug Alcohol Rev. 2006;25(1):216.

329. de Vries H, Kremers SP, Smeets T, Brug J, Eijmael K. The effectiveness of tailored feedback and action plans in an intervention addressing multiple health behaviors. Am J Health Promot. 2008;22(6):417-25.

330. Dijkstra A, De Vries H, Bakker M. Pros and cons of quitting, self-efficacy, and the stages of change in smoking cessation. Journal of consulting and clinical psychology. 1996;64(4):758.

331. Bangor A, Kortum PT, Miller JT. An empirical evaluation of the system usability scale. Intl Journal of HumanComputer Interaction. 2008;24(6):574-94.

332. O'Connor A. User manual-Decisional Conflict Scale (16-item statement format);@ 1993 (updated 2010). Retrieved from Ottawa Hospital Research Institute website http://decisionaid ohri ca/docs/develop/ User_Manuals/UM_Decisional_Conflict pdf. 2010.

333. Bangor A, Kortum P, Miller J. Determining what individual SUS scores mean: Adding an adjective rating scale. Journal of usability studies. 2009;4(3):114-23. 
334. O'Connor A. User manual-decisional conflict scale. 2010. Ref Type: Online Source2017.

335. Glanz K, Rimer BK, Viswanath K. Health behavior and health education: theory, research, and practice: John Wiley \& Sons; 2008.

336. DiClemente CC, Prochaska JO, Fairhurst SK, Velicer WF, Velasquez MM, Rossi JS. The process of smoking cessation: an analysis of precontemplation, contemplation, and preparation stages of change. J Consult Clin Psychol. 1991;59(2):295-304.

337. Dijkstra A, De Vries H, Roijackers J, van Breukelen G. Tailored interventions to communicate stage-matched information to smokers in different motivational stages. J Consult Clin Psychol. 1998;66(3):549-57.

338. Soetens KC, Vandelanotte $\mathrm{C}$, de Vries $\mathrm{H}$, Mummery KW. Using online computer tailoring to promote physical activity: a randomized trial of text, video, and combined intervention delivery modes. Journal of Health Communication. 2014;19(12):1377-92.

339. Stanczyk NE, Smit ES, Schulz DN, de Vries H, Bolman C, Muris JW, et al. An economic evaluation of a videoand text-based computer-tailored intervention for smoking cessation: a cost-effectiveness and cost-utility analysis of a randomized controlled trial. PLoS One. 2014;9(10):e110117.

340. Smit ES, Evers SM, de Vries H, Hoving C. Cost-effectiveness and cost-utility of Internet-based computer tailoring for smoking cessation. J Med Internet Res. 2013;15(3):e57.

341. Smit ES, Candel MJ, Hoving C, de Vries H. Results of the PAS Study: A Randomized Controlled Trial Evaluating the Effectiveness of a Web-Based Multiple Tailored Smoking Cessation Program Combined With Tailored Counseling by Practice Nurses. Health Commun. 2016;31(9):1165-73.

342. Bouter LM, Dongen MCJM, Zielhuis GA. Epidemiologisch onderzoek: opzet en interpretatie: Springer; 2006.

343. Gultzow T, Smit ES, Hudales R, Knapen V, Rademakers J, Dirksen CD, et al. An Autonomy-Supportive Online Decision Aid to Assist Smokers in Choosing Evidence-Based Cessation Assistance: Development Process and Protocol of a Randomized Controlled Trial. JMIR Res Protoc. 2020;9(12):e21772.

344. Altendorf M, Hoving C, Van Weert JC, Smit ES. Effectiveness of Message Frame-Tailoring in a Web-Based Smoking Cessation Program: Randomized Controlled Trial. J Med Internet Res. 2020;22(4):e17251.

345. Cheung KL, Schwabe I, Walthouwer MJL, Oenema A, Lechner L, de Vries H. Effectiveness of a Video-Versus Text-Based Computer-Tailored Intervention for Obesity Prevention after One Year: A Randomized Controlled Trial. Int J Environ Res Public Health. 2017;14(10):1275.

346. Elling JM, De Vries H. Influence of Animation- Versus Text-Based Delivery of a Web-Based ComputerTailored Smoking Cessation Intervention on User Perceptions. European Journal of Health Communication. 2021;2(3):1-23.

347. Wentzel J, van der Vaart R, Bohlmeijer ET, van Gemert-Pijnen JE. Mixing Online and Face-to-Face Therapy: How to Benefit From Blended Care in Mental Health Care. JMIR Ment Health. 2016;3(1):e9.

348. Bricca A, Swithenbank Z, Scott N, Treweek S, Johnston M, Black N, et al. Predictors of recruitment and retention in randomized controlled trials of behavioural smoking cessation interventions: a systematic review and meta-regression analysis. Addiction. 2021(n/a).

349. Bennett GG, Glasgow RE. The delivery of public health interventions via the Internet: actualizing their potential. Annual review of public health. 2009;30:273-92.

350. Ryan RM, Deci EL. Self-determination theory and the facilitation of intrinsic motivation, social development, and well-being. American psychologist. 2000;55(1):68.

351. Green LV, Savin S, Lu Y. Primary care physician shortages could be eliminated through use of teams, nonphysicians, and electronic communication. Health Affairs. 2013;32(1):11-9.

352. Lockwood C. Nurses as substitutes for doctors in primary care. International journal of nursing studies. 2020;106:103362.

353. Laurant MG, Hermens RP, Braspenning JC, Sibbald B, Grol RP. Impact of nurse practitioners on workload of general practitioners: randomised controlled trial. BMJ (Clinical research ed). 2004;328(7445):927.

354. Hoving C, Mudde AN, de Vries $\mathrm{H}$. Effect of recruitment method and setting on the composition of samples consisting of adult smokers. Patient Educ Couns. 2007;65(1):79-86.

355. Salmon P, Peters S, Rogers A, Gask L, Clifford R, Iredale W, et al. Peering through the barriers in GPs' explanations for declining to participate in research: the role of professional autonomy and the economy of time. Fam Pract. 2007;24(3):269-75.

356. Ariens LF, Schussler-Raymakers FM, Frima C, Flinterman A, Hamminga E, Arents BW, et al. Barriers and facilitators to eHealth use in daily practice: perspectives of patients and professionals in dermatology. Journal of medical Internet research. 2017;19(9):e300. 


\section{APPENDICES}

357. van de Glind I, Heinen M, Geense W, Mesters I, Wensing M, van Achterberg T. Making the connection-factors influencing implementation of evidence supported and non-evaluated lifestyle interventions in healthcare: a multiple case study. Health Educ Res. 2015;30(4):521-41.

358. Neff R, Fry J. Periodic prompts and reminders in health promotion and health behavior interventions: systematic review. Journal of medical Internet research. 2009;11(2):e16.

359. Yardley L, Spring BJ, Riper H, Morrison LG, Crane DH, Curtis K, et al. Understanding and Promoting Effective Engagement With Digital Behavior Change Interventions. Am J Prev Med. 2016;51(5):833-42.

360. van Westen-Lagerweij NA, Meeuwsen EG, Croes EA, Meijer E, Chavannes NH, Willemsen MC. The referral of patients to smoking cessation counselling: perceptions and experiences of healthcare providers in general practice. BMC health services research. 2021;21(1):583.

361. van Eerd EA, van Rossem CR, Spigt MG, Wesseling G, van Schayck OC, Kotz D. Do we need tailored smoking cessation interventions for smokers with COPD? A comparative study of smokers with and without COPD regarding factors associated with tobacco smoking. Respiration. 2015;90(3):211-9.

362. Tonnesen P, Carrozzi L, Fagerstrom KO, Gratziou C, Jimenez-Ruiz C, Nardini S, et al. Smoking cessation in patients with respiratory diseases: a high priority, integral component of therapy. The European respiratory journal. 2007;29(2):390-417.

363. de Hoog N, Bolman C, Berndt N, Kers E, Mudde A, de Vries H, et al. Smoking cessation in cardiac patients: the influence of action plans, coping plans and self-efficacy on quitting smoking. Health Educ Res. 2016;31(3):35062.

364. Papadakis S, Tulloch HE, Gharib M, Pipe AL. Profile of tobacco users identified in primary care practice and predictors of readiness to quit: a cross-sectional survey. CMAJ Open. 2016;4(1):E41-7.

365. van Gelder MM, Bretveld RW, Roeleveld N. Web-based questionnaires: the future in epidemiology? American journal of epidemiology. 2010;172(11):1292-8.

366. Page SJ, Persch AC. Recruitment, retention, and blinding in clinical trials. Am J Occup Ther. 2013;67(2):15461.

367. Hilberink SR, Jacobs JE, Schlosser M, Grol RP, de Vries H. Characteristics of patients with COPD in three motivational stages related to smoking cessation. Patient Educ Couns. 2006;61(3):449-57.

368. Butler CC, Pill R, Stott NC. Qualitative study of patients' perceptions of doctors' advice to quit smoking: implications for opportunistic health promotion. BMJ (Clinical research ed). 1998;316(7148):1878-81.

369. Van Eerd EAM, Risør MB, Spigt M, Godycki-Cwirko M, Andreeva E, Francis N, et al. Why do physicians lack engagement with smoking cessation treatment in their COPD patients? A multinational qualitative study. NPJ primary care respiratory medicine. 2017;27(1):1-6.

370. van Eerd EA, van der Meer RM, van Schayck OC, Kotz D. Smoking cessation for people with chronic obstructive pulmonary disease. Cochrane Database Syst Rev. 2016(8):CD010744.

371. Hiscock R, Dobbie F, Bauld L. Smoking Cessation and Socioeconomic Status: An Update of Existing Evidence from a National Evaluation of English Stop Smoking Services. BioMed research international. 2015;2015:274056.

372. Kock L, Brown J, Hiscock R, Tattan-Birch H, Smith C, Shahab L. Individual-level behavioural smoking cessation interventions tailored for disadvantaged socioeconomic position: a systematic review and meta-regression. Lancet Public Health. 2019;4(12):e628-e44.

373. Vangeli E, Stapleton J, Smit ES, Borland R, West R. Predictors of attempts to stop smoking and their success in adult general population samples: a systematic review. Addiction. 2011;106(12):2110-21.

374. Hunt DP. The concept of knowledge and how to measure it. Journal of intellectual capital. 2003.

375. Douma L. Colorectal cancer screening: Yes or No?: Insights into public opinion and the individual decisionmaking process. 2019.

376. O'Connor AM, Tugwell P, Wells GA, Elmslie T, Jolly E, Hollingworth G, et al. A decision aid for women considering hormone therapy after menopause: decision support framework and evaluation. Patient Educ Couns. 1998;33(3):267-79.

377. O'Connor AM, Tugwell P, Wells GA, Elmslie T, Jolly E, Hollingworth G, et al. Randomized trial of a portable, self-administered decision aid for postmenopausal women considering long-term preventive hormone therapy. Medical decision making : an international journal of the Society for Medical Decision Making. 1998;18(3):295-303.

378. Man-Son-Hing M, Laupacis A, O'Connor AM, Biggs J, Drake E, Yetisir E, et al. A patient decision aid regarding antithrombotic therapy for stroke prevention in atrial fibrillation: a randomized controlled trial. Jama. 1999;282(8):737-43. 
379. Fiset V, O'Connor AM, Evans W, Graham I, Degrasse C, Logan J. Development and evaluation of a decision aid for patients with stage IV non-small cell lung cancer. Health expectations : an international journal of public participation in health care and health policy. 2000;3(2):125-36.

380. Grant FC, Laupacis A, O'Connor AM, Rubens F, Robblee J. Evaluation of a decision aid for patients considering autologous blood donation before open-heart surgery. $\mathrm{CMAJ}$ : Canadian Medical Association journal = journal de l'Association medicale canadienne. 2001;164(8):1139-44.

381. Mitchell SL, Tetroe J, O'Connor AM. A decision aid for long-term tube feeding in cognitively impaired older persons. Journal of the American Geriatrics Society. 2001;49(3):313-6.

382. Cranney A, O'Connor AM, Jacobsen MJ, Tugwell P, Adachi JD, Ooi DS, et al. Development and pilot testing of a decision aid for postmenopausal women with osteoporosis. Patient Educ Couns. 2002;47(3):245-55.

383. Stacey D, O'Connor AM, DeGrasse C, Verma S. Development and evaluation of a breast cancer prevention decision aid for higher-risk women. Health expectations : an international journal of public participation in health care and health policy. 2003;6(1):3-18

384. Ng JYY, Ntoumanis N, Thøgersen-Ntoumani C, Deci EL, Ryan RM, Duda JL, et al. Self-Determination Theory Applied to Health Contexts:A Meta-Analysis. Perspectives on Psychological Science. 2012;7(4):325-40.

385. Williams GC, Cox EM, Kouides R, Deci EL. Presenting the facts about smoking to adolescents: effects of an autonomy-supportive style. Arch Pediatr Adolesc Med. 1999;153(9):959-64.

386. Williams GC, Gagné M, Ryan RM, Deci EL. Facilitating autonomous motivation for smoking cessation. Health psychology: official journal of the Division of Health Psychology, American Psychological Association. 2002;21(1):40-50.

387. Smit ES, Dima AL, Immerzeel SAM, van den Putte B, Williams GC. The Virtual Care Climate Questionnaire: Development and Validation of a Questionnaire Measuring Perceived Support for Autonomy in a Virtual Care Setting. J Med Internet Res. 2017;19(5):e155.

388. Bengoa R. Transforming health care: an approach to system-wide implementation. Int J Integr Care. 2013;13:e039.

389. Green LW. Making research relevant: if it is an evidence-based practice, where's the practice-based evidence? Family practice. 2008;25(suppl_1):i20-i4.

390. Ramaswamy V, Ozcan K. The co-creation paradigm. Stanford: Stanford University Press; 2020.

391. van Rossem C, Spigt M, Smit ES, Viechtbauer W, Mijnheer KK, van Schayck CP, et al. Combining intensive practice nurse counselling or brief general practitioner advice with varenicline for smoking cessation in primary care: study protocol of a pragmatic randomized controlled trial. Contemporary clinical trials. 2015;41:298-312.

392. Sturgiss EA, Douglas K. A collaborative process for developing a weight management toolkit for general practitioners in Australia-an intervention development study using the Knowledge To Action framework. Pilot Feasibility Stud. 2016;2(1):20.

393. Janamian T, Crossland L, Jackson CL. Embracing value co-creation in primary care services research: a framework for success. The Medical journal of Australia. 2016;204(7 Suppl):S5-11.

394. Cook DA, Levinson AJ, Garside S, Dupras DM, Erwin PJ, Montori VM. Instructional design variations in internet-based learning for health professions education: a systematic review and meta-analysis. Acad Med. 2010;85(5):909-22.

395. Glasgow RE, Vogt TM, Boles SM. Evaluating the public health impact of health promotion interventions: the RE-AIM framework. Am J Public Health. 1999;89(9):1322-7.

396. Reif S, Horgan CM, Ritter GA. Treatment services: triangulation of methods when there is no gold standard. Substance use \& misuse. 2011;46(5):620-32.

397. Miller WR, Rollnick S. Motivational interviewing: Preparing people for change. New York: Guilford Publications; 2002.

398. Hall K, Gibbie T, Lubman DI. Motivational interviewing techniques - facilitating behaviour change in the general practice setting. Aust Fam Physician. 2012;41(9):660-7

399. Karaman S. Nurses' perceptions of online continuing education. BMC Med Educ. 2011;11(1):86.

400. Voogdt-Pruis HR, Van Ree JW, Gorgels AP, Beusmans GH. Adherence to a guideline on cardiovascular prevention: a comparison between general practitioners and practice nurses. International journal of nursing studies. 2011;48(7):798-807.

401. Heinrich E, Candel MJ, Schaper NC, de Vries NK. Effect evaluation of a Motivational Interviewing based counselling strategy in diabetes care. Diabetes research and clinical practice. 2010;90(3):270-8. 


\section{APPENDICES}

402. Noordman J, van Lee I, Nielen M, Vlek H, van Weijden T, van Dulmen S. Do trained practice nurses apply motivational interviewing techniques in primary care consultations? Journal of Clinical Medicine Research. 2012;4(6):393.

403. Efraimsson EÖ, Klang B, Ehrenberg A, Larsson K, Fossum B, Olai L. Nurses' and patients' communication in smoking cessation at nurse-led COPD clinics in primary health care. European clinical respiratory journal. 2015;2(1):27915.

404. De Vries H, Mudde AN, Dijkstra A, Willemsen MC. Differential beliefs, perceived social influences, and self-efficacy expectations among smokers in various motivational phases. Prev Med. 1998;27(5 Pt 1):681-9.

405. Michie S, Churchill S, West R. Identifying evidence-based competences required to deliver behavioural support for smoking cessation. Ann Behav Med. 2011;41(1):59-70.

406. Kastaun S, Leve V, Hildebrandt J, Funke C, Klosterhalfen S, Lubisch D, et al. Training general practitioners in the $A B C$ versus $5 A$ s method of delivering stop-smoking advice: a pragmatic, two-arm cluster randomised controlled trial. ERJ Open Res. 2021;7(3).

407. Papadakis S, Anastasaki M, Papadakaki M, Antonopoulou M, Chliveros C, Daskalaki C, et al. 'Very brief advice' (VBA) on smoking in family practice: a qualitative evaluation of the tobacco user's perspective. BMC family practice. 2020;21(1):121.

408. van der Kleij R, Kasteleyn MJ, Meijer E, Bonten TN, Houwink EJF, Teichert M, et al. SERIES: eHealth in primary care. Part 1: Concepts, conditions and challenges. Eur J Gen Pract. 2019;25(4):179-89.

409. van der Vaart R, Witting M, Riper H, Kooistra L, Bohlmeijer ET, van Gemert-Pijnen LJ. Blending online therapy into regular face-to-face therapy for depression: content, ratio and preconditions according to patients and therapists using a Delphi study. BMC psychiatry. 2014;14(1):1-10.

410. Talboom-Kamp EP, Verdijk NA, Kasteleyn MJ, Numans ME, Chavannes NH. From chronic disease management to person-centered eHealth; a review on the necessity for blended care. Clinical eHealth. 2018;1(1):3-7.

411. Smeets $\mathrm{O}$, Zijlstra-Vlasveld M. Blended e-(mental) health in de huisartsenpraktijk. Utrecht: Trimbos-institute; 2016.

412. Siemer L, Pieterse ME, Brusse-Keizer MGJ, Postel MG, Ben Allouch S, Sanderman R. Study protocol for a non-inferiority trial of a blended smoking cessation treatment versus face-to-face treatment (LiveSmokefreeStudy). BMC Public Health. 2016;16(1):1187.

413. Graham A, Milner P, Saul J, Pfaff L. Online advertising as a public health and recruitment tool: comparison of different media campaigns to increase demand for smoking cessation interventions. Journal of Medical Internet Research. 2008;10(5):e50.

414. Gultzow T, Smit ES, Hudales R, Dirksen CD, Hoving C. Smoker profiles and their influence on smokers' intention to use a digital decision aid aimed at the uptake of evidence-based smoking cessation tools: An explorative study. Digit Health. 2020;6:2055207620980241.

415. Wipfli HL, Fujimoto K, Valente TW. Global tobacco control diffusion: the case of the framework convention on tobacco control. Am J Public Health. 2010;100(7):1260-6.

416. Yach D. The origins, development, effects, and future of the WHO Framework Convention on Tobacco Control: a personal perspective. Lancet (London, England). 2014;383(9930):1771-9.

417. Meijer E, Kampman M, Geisler MS, Chavannes NH. "It's on everyone's plate": a qualitative study into physicians' perceptions of responsibility for smoking cessation. Substance abuse treatment, prevention, and policy. 2018;13(1):48.

418. Hassel Dv, Korevaar J, Batenburg R, Schellevis F. De Toekomstvisie Huisartsenzorg 2022, waar staat de huisartsenzorg anno 2014? Utrecht: NIVEL; 2015.

419. Brug J. Gezondheidsvoorlichting en gedragsverandering: Uitgeverij Van Gorcum; 2010.

420. Twardella $\mathrm{D}$, Brenner $\mathrm{H}$. Effects of practitioner education, practitioner payment and reimbursement of patients' drug costs on smoking cessation in primary care: a cluster randomised trial. Tob Control. 2007;16(1):15-21.

421. Ekeland AG, Bowes A, Flottorp S. Effectiveness of telemedicine: a systematic review of reviews. International journal of medical informatics. 2010;79(11):736-71.

422. Elbert NJ, van Os-Medendorp H, van Renselaar W, Ekeland AG, Hakkaart-van Roijen L, Raat $H$, et al. Effectiveness and cost-effectiveness of ehealth interventions in somatic diseases: a systematic review of systematic reviews and meta-analyses. J Med Internet Res. 2014;16(4):e110.

423. Gomez Quinonez S, Walthouwer MJ, Schulz DN, de Vries H. mHealth or eHealth? Efficacy, Use, and Appreciation of a Web-Based Computer-Tailored Physical Activity Intervention for Dutch Adults: A Randomized Controlled Trial. J Med Internet Res. 2016;18(11):e278. 
424. Ghorai K, Akter S, Khatun F, Ray P. mHealth for Smoking Cessation Programs: A Systematic Review. J Pers Med. 2014;4(3):412-23.

425. Brose LS, West R, McDermott MS, Fidler JA, Croghan E, McEwen A. What makes for an effective stop-smoking service? Thorax. 2011;66(10):924-6.

426. Kerngroep Zorgstandaard Tabaksverslaving. Zorgstandaard Tabaksverslaving. Utrecht: Partnership Stop met Roken; 2019. 


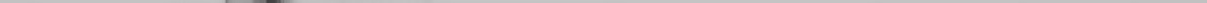




\section{SUMMARY}

Smoking is still one of the leading causes of illness and premature death among the general population, but especially among groups with a lower socioeconomic status. It is therefore crucial to improve smoking cessation strategies. The primary care setting (PCS) can play a key role in reaching smokers because of its strategic position in the health-care system and the existence of guidelines aimed at providing evidence based smoking cessation care by practice nurses (PNs) and other primary care professionals (PCPs). These guidelines recommend discussing the use of evidence-based smoking cessation interventions (EBSCIs) such as behavioral counseling (face-to-face counseling, eHealth, group counseling, or counseling over the telephone) and pharmacological supplementations (nicotine replacement therapy or pharmacotherapy in the form of non-nicotine medication). Using EBSCls can double the chance of success of a smoking cessation attempt, but unfortunately, EBSCls are still structurally underused. To increase the use of EBSCls and to support referral by PCPs to EBSCls during consultations, a referral aid (RA) for smoking cessation interventions for smoking patients within the PCS was developed. The overall aim of the dissertation is to describe the process of the development of this RA and to investigate its potential.

Chapter 1 provides a general introduction to Chapters 2-7 of this dissertation, including important background information, details on the theoretical groundings, and the research questions of the studies reported on in this dissertation. Chapters 2-5 describe the potential of the RA in the PCS, and Chapters 6 and 7 explore further applications of the RA.

\section{Part I: The potential of an RA in the PCS}

Chapter 2 presents a Delphi study that aimed to obtain an overview of the knowledge and viewpoints on the effectiveness and use of EBSCls among PCPs. A three-round online Delphi study was conducted among researchers and PCPs to gain an overview of (1) the criteria that are important for recommending EBSCls, (2) the perceptions of both groups on the effectiveness of EBSCls, (3) the factors to consider when counseling different (highrisk) groups of smokers, and (4) the perceptions of both groups on the use of e-cigarettes as an EBSCI. We found a high level of agreement within the groups on which characteristics of smokers should be considered when recommending an EBSCI. We also found that PCPS displayed a lower consensus on the effectiveness of EBSCls. Both groups valued the use of special protocols for different (high-risk) groups of patients, but the two groups did not reach a consensus on the use of e-cigarettes as an effective means to quit. This inventory of PCPs' needs regarding EBSCls, and their usage provided us with clear directions to facilitate a higher uptake of and referral to EBSCIs in the PCS. 


\section{APPENDICES}

Chapter 3 describes the study protocol of the RA, including the RAs development and study design of the randomized controlled trial (RCT). The RA was named the "StopWijzer," which can be translated as either "Stop guide" or "Stop smarter." The RA aimed to help PNs, and smokers identify an individual patient's preferred method for quitting smoking, and the aim of developing this resource was to increase the use of EBSCls for smoking cessation. The PNs in the experimental condition received an intervention manual to aid them in discussing smoking cessation with their patients and to help them select an EBSCl that fits patients' needs and preferences. Smoking cessation interventions included in the RA are (1) faceto-face counseling, (2) counseling via the Internet (eHealth), (3) telephone counseling, (4) group counseling, (5) pharmacotherapy, and (6) nicotine replacement therapy. The latter two were preferably combined with a counseling method. Patients who agreed to participate in the study were counseled in accordance with the RA and were stimulated to use an included $\mathrm{EBSCl}$ to quit. The principal component was an instruction manual for using the RA, which was also available online. Additional elements were a handout with flowcharts from the manual, a placemat with an overview of all available EBSCls, and several promotion materials (flyers, posters, business cards, notebook, and pen).

Chapter 4 presents the results of the process and effect evaluation among smoking patients recruited through PNs in the PCS throughout the Netherlands. The aim of this study was to explore the use, appreciation, and effects of the RA from the perspective of two user groups: (1) PNs ( $n=73$ ) and (2) smokers ( $n=285)$. To optimally explore the experiences of both groups, two studies were conducted, namely (1) an RCT among smoking patients recruited by $\mathrm{PNs}$ and (2) a process evaluation among a subgroup of these PNs that participated in the trial to investigate the course of the recruitment process. In both groups, the response was low. Overall, PNs found the RA materials to be clear and understandable. Smokers had similar but (slightly) fewer positive opinions. However, the RA was not intensively used, and the experimental groups of smokers did not differ in their rate of smoking abstinence compared with the control group. Since the RA was well-received by both PNs and smokers, the RA is potentially suitable for implementation in the PCS. We concluded that further research should be aimed at determining how to facilitate the adoption of the RA within the PCS as well as how to better involve PNs and smokers when recruiting for an RCT and at how to foster effective counseling and referral to EBSCIs.

Chapter 5 presents the factors associated with the intention to adopt an RA facilitating the referral to EBSCIs by PCPs in charge of smoking counseling in the PCS (partly from the main study described in Chapter 4 and partly newly gained). Participants $(n=85)$ were recruited for a cross-sectional study from June to September 2020 and were asked to fill in online questionnaires that were based on the I-Change Model. T-tests were used to compare adopters $(n=37)$ with nonadopters $(n=48)$ on predisposing (demographics), 
motivational (attitude, social support, and self-efficacy), and post-motivational (perceived skills and barriers) factors. Logistic regression analyses were conducted to explore factors explaining the intention to adopt. Although appreciation was high in both groups, most PCPs did not intend to adopt the RA ( $>50 \%$ ). Nonadopters reported an overall more negative attitude toward the RA than adopters by perceiving fewer advantages and more disadvantages, experienced less social support, and had a low level of self-efficacy. They also experienced more barriers such as a lack of time and a lack of skills. These factors were also associated with the intention to adopt. Recommendations for future adoption include improving the tool itself through a second round of co-creation focusing on the adoptability of the RA in practice. A second recommendation pertains to communicating the added value of referring to EBSCls and integrating the RA's use in smoking cessation training for PCPs. Doing so may help to increase a positive attitude, social support, selfefficacy, and perceived skills toward using the RA among PCPs.

\section{Part II: Future applications and possibilities}

Chapter 6 describes the outcomes of a scoping review that explored the literature regarding decision aids (DAs) supporting decision making about diet, physical activity, sleep, and substance use, including smoking cessation. Interventions had to (1) support informed decision making and (2) provide information and assist in choosing between a minimum of two options. Thirty-five scientific articles and four DAs (gray literature) were included, among which 29 (94\%) described substance use. All DAs offered users information and possibilities for value and/or preference clarification as well as many other elements, such as goal setting. Few articles used standardized measures, such as decisional conflict $(n=$ $4,13 \%$ ). Although the review only found some positive behavioral effects of the use of DAs, this study contributes to charting the existing decision aids and RAs in health promotion, their behavioral effects, and the areas of improvement (e.g., effective intervention elements and development).

Chapter 7 describes a freestanding usability study conducted in September 2020 on a standalone version of the RA (DA) with smokers $(n=497)$ recruited through an online research panel using a cross-sectional design. The aim of this study was threefold: (1) to conduct a usability evaluation of a standalone version of the RA; 2 ) to evaluate the level of appreciation and informed decision making after using the RA; and (3) to determine a possible change in the intention to use EBSCls before and directly after reviewing the DA. T-tests and Chi-square tests were conducted to test the differences between smokers who differed in perceived usability of the DA in program appreciation and in decisional conflict. Most participants evaluated the usability of the DA as moderate (MU; $n=393,79.1 \%$ ) or good (GU; $n=104,20.9 \%$ ); by contrast, those who intended to quit found it more usable. Most Participants found the DA only moderately usable, although those who found it more 


\section{APPENDICES}

usable often had a significant higher intention to quit. Participants who found the usability of the DA to be good rated all elements higher concerning the evaluation of the DA (including recommendation to others and overall mark) and experienced less decisional conflict with regard to choosing a potential EBSCI after reviewing the DA. Furthermore, after reviewing the DA, participants on average had a significantly higher intention to use more EBSCls, particularly in the form of eHealth. We concluded that the RA can be of use to smokers who have an intention to quit smoking. Recommendations for making the DA more usable and well-received among a broader group of smokers include performing tailoring, transforming text-based information into video-based information, and including value-clarification methods. Furthermore, as the DA was only found to be moderately usable in the standalone version, a hybrid variant where smokers can use the DA on their own and with the guidance of a PCP could aid both groups in choosing a fitting EBSCI option.

Finally, Chapter 8 discusses the results presented in Chapters 2-6 of the dissertation and contains some considerations of the studies described above, including recommendations for future research and practice. The goal of the research was to develop an RA to help PCPs refer to EBSCls in order to increase the use of EBSCls among smokers. The studies described in this dissertation show that the RA was well appreciated among PCPs and that its use led to slightly more discussion and referral to EBSCls. The RA was also appreciated by smokers, especially those who are motivated to quit smoking. Although the RA introduced a wide range of EBSCls, this did not lead to decisional conflict among its users. However, no effects on smoking abstinence could be reported. This was most probably caused by both conditions receiving evidence-based counseling by their PN and the small study sample. Furthermore, the RA was not intensively used during the RCT, which was probably cased by barriers such as lack of time and a high workload. In this chapter we addressed some considerations regarding facilitating the recruitment of PCPs and smoking patients and their adherence to the RA. We also stated some recommendation for further research related to (1) the PCS (e.g., potentially increasing participation and improving implementation); (2) the RA described in this dissertation (e.g., developing a hybrid variant and supplementing the RA with various forms of motivational techniques, tailoring, videobased materials, and value-clarification exercises); and (3) smoking cessation in general (addressing the health insurance system and policy makers and improving the availability of and access to EBSCls).

Overall, to increase the use of EBSCIS and implement the RA in the daily routine of PCPs, we can see the potential of the RA as described in this study in the PCs, for example as part of the Ask-Advise-Refer strategy. The available EBSCls should be explained to smokers during the refer phase, or the RA should be used as a reference that smokers can consult themselves after talking to their PCP, which could decrease active counseling time. In order to stimulate implementation, the RA could therefore be included in national smoking 
cessation trainings aimed at PCPs. Furthermore, the development of a hybrid variant can support the use of the RA in preparation for a consultation with a PCP or allow the smoker to make their own choice regarding the use of EBSCls in case the tool is offered online as a self-help tool. 


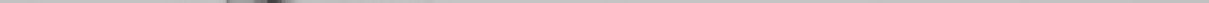




\section{SAMENVATTING}

Roken is nog steeds een van de belangrijkste oorzaken van ziekte en vroegtijdig overlijden onder de algemene bevolking, maar vooral onder groepen met een lagere sociaaleconomische status (SES). Het is daarom belangrijk om de bestaande stoppen-met-rokenstrategieën te verbeteren. Vanwege de strategische positie in het gezondheidssysteem kan de eerstelijnszorg bijdragen in het bereiken van rokers. Ook beschikt de eerstelijnszorg over duidelijke richtlijnen gericht op het verbeteren van de effectieve stoppen-met-rokenzorg door praktijkondersteuners (POHs) en andere (eerstelijns-)zorgprofessionals (ZPs). In deze richtlijnen wordt ook gesproken over het gebruiken van evidence-based stoppen-met-rokeninterventies (EBSMRIs). Deze EBSMRIs bestaan uit vormen van gedragscounseling (faceto-facecounseling, eHealth, groepscounseling of counseling via de telefoon) en uit farmacotherapie (nicotinevervangende middelen of SMR-medicatie). Het gebruik van EBSMRIs kan de kans op succes van een stoppoging verdubbelen, maar helaas worden EBSMRIs structureel onderbenut. Om POHs te ondersteunen bij het bespreken van EBSMRIs en hiermee het gebruik van EBSMRIs te verhogen is een verwijshulp ontwikkeld. Het algemene doel van dit proefschrift is het beschrijven van het ontstaan van deze verwijshulp en het onderzoeken van de mogelijkheden ervan.

In hoofdstuk 1 wordt een algemene inleiding gegeven op de hoofdstukken 2 - 7 van dit proefschrift, met inbegrip van achtergrondinformatie, de theoretische onderbouwingen en de onderzoeksvragen van de studies in dit proefschrift. In hoofdstukken 2 - 5 wordt vervolgens de potentie van een verwijshulp in de huisartspraktijk beschreven, waarna in hoofdstukken 6 en 7 mogelijke andere toepassingen van de verwijshulp geëxploreerd worden.

\section{Deel I: Het potentieel van een verwijshulp in de eerstelijnsgezondheidszorg}

In hoofdstuk 2 wordt een Delphi-studie gepresenteerd waarin de kennis en standpunten van professionals in de eerstelijnsgezondheidszorg met betrekking tot het gebruik en de doeltreffendheid van EBSMRIs wordt beschreven. Een online Delphi-studie van drie ronden is uitgevoerd onder onderzoekers en ZPs om een overzicht te verkrijgen van 1) de criteria die belangrijk zijn voor het aanbevelen van SMR-interventies, 2) de visies van beide groepen op de effectiviteit van SMR-interventies, 3) de factoren waarmee rekening gehouden moet worden bij het begeleiden van verschillende (hoogrisico)groepen rokers en 4) de visies op het gebruik van e-sigaretten als een hulpmiddel om te stoppen. Beide groepen vertoonden een hoge mate van overeenstemming omtrent karakteristieken van rokers die in aanmerking dienen genomen te worden bij het selecteren van een te adviseren EBSMRI. Voorts bleken de ZPs een lagere mate van consensus te vertonen over de doeltreffendheid van EBSMRIs. Daarnaast waardeerden beide groepen het gebruik van speciale protocollen voor 


\section{APPENDICES}

verschillende (hoogrisico)groepen patiënten, maar bereikten geen consensus over het gebruik van e-sigaretten als stophulpmiddel. Deze inventarisatie van de behoeften van ZPs met betrekking tot EBSMRIs gaf ons een beter inzicht in de wijze waarop het gebruik van en verwijzen naar EBSMRIs in de eerstelijnszorg gefaciliteerd kan worden.

Hoofdstuk 3 beschrijft het onderzoeksprotocol van de verwijshulp (de StopWijzer), inclusief de ontwikkeling en de opzet van het gerandomiseerde onderzoek met zowel een experimentele- als controlegroep om de effectiviteit van de verwijshulp te toetsen. Het doel van de verwijshulp was het ondersteunen van $\mathrm{POH}$ bij het verwijzen van rokende patienten naar EBSMRIs en daardoor het gebruik van EBSMRIs te verhogen. De POHs in de experimentele groep ontvingen een handleiding voor de interventie met het doel om hen te ondersteunen bij het SMR-gesprek met hun patiënten en het selecteren van een EBSMRI die aansluit op de behoeften en voorkeuren van de patiënt. EBSMRIs die in de verwijshulp waren opgenomen zijn 1) face-to-facecounseling, 2) counseling via internet (eHealth), 3) telefonische counseling, 4) groepscounseling, 5) farmacotherapie en 6) nicotinevervangingstherapie. De laatste twee werden bij voorkeur gecombineerd met een gedragsveranderingsmethode. Patiënten die instemden met deelname aan het onderzoek werden conform de verwijshulp begeleid. Naast de eerdergenoemde handleiding, die het belangrijkste onderdeel van de verwijshulp was en ook online te bekijken, ontvingen deelnemende $\mathrm{POHs}$ ook aanvullende elementen in de vorm van een hand-out met de in de handleiding weergegeven stroomdiagrammen, een placemat met een overzicht van alle beschikbare EBSMRIs en diverse promotiematerialen (flyers, posters, visitekaartjes, notitieboek en pen).

Hoofdstuk 4 presenteert de resultaten van de evaluatie van het proces en de effectiviteit van de StopWijzer onder rokende patiënten die door POHs zijn geworven in de eerstelijnszorg in heel Nederland. Het doel van deze studie was om het gebruik, de waardering en de effecten van de verwijshulp te onderzoeken vanuit het perspectief van twee gebruikersgroepen: 1$) \mathrm{POHs}(n=73)$ en 2$)$ rokers $(n=285)$. Om de ervaringen van beide groepen optimaal in kaart te brengen zijn twee verschillende onderzoeken uitgevoerd; namelijk 1) een gerandomiseerde gecontroleerde studie onder rokende patiënten die door $\mathrm{POH}$ zijn geworven en 2) een procesevaluatie onder een subgroep van deze $\mathrm{POH}$ om het verloop van het wervingsproces te onderzoeken. In beide groepen was de respons laag. Over het algemeen vonden POHs de StopWijzermaterialen duidelijk en begrijpelijk. Rokers hadden doorgaans een vergelijkbaar maar (iets) minder positief oordeel. De verwijshulp werd echter niet intensief gebruikt, en de rokers in de experimentele groepen verschilden niet in hun mate van rook abstinentie in vergelijking met de rokers in de controlegroep. Aangezien de verwijshulp wel goed werd ontvangen door zowel POHs als rokers en er een hoger aantal EBSMRIs werd gebruikt in de experimentele conditie (vooral in de vorm van 
eHealth, groepsconsultatie en nicotinevervangingstherapie, waar deze toename significant was), is de verwijshulp mogelijk geschikt voor implementatie in de eerstelijnszorg. Wij concluderen dat vervolgonderzoek gericht moet zijn op het beoordelen van 1) hoe de adoptie van de verwijshulp binnen de eerstelijnszorg kan worden vergemakkelijkt en 2) hoe POHs en rokers beter kunnen worden betrokken bij de werving voor een RCT en hoe effectieve begeleiding en verwijzing naar EBSMRIs kunnen worden bevorderd.

Hoofdstuk 5 presenteert factoren die samenhangen met de intentie van ZPs die verantwoordelijk zijn voor SMR-begeleiding in de huisartsenpraktijk om een verwijshulp ter bevordering van het gebruik van EBSMRIs te adopteren. Deelnemers $(n=85$; deels geworven onder deelnemers aan het hoofdonderzoek beschreven in hoofdstuk 4 en deels nieuw geworven) werden geworven voor een cross-sectioneelonderzoek van juni tot september 2020 en werden gevraagd om online vragenlijsten in te vullen die gebaseerd waren op het I-Change Model. T-tests werden gebruikt om adoptanten $(n=37)$ te vergelijken met nietadoptanten ( $n=48$ ) op predisponerende (demografisch), motivationele (attitude, sociale steun en zelfeffectiviteit) en post-motivationele (waargenomen vaardigheden en barrières) factoren. Hoewel de waardering voor de verwijshulp in beide groepen hoog was, waren de meeste ZPs niet van plan om deze te adopteren (>50\%). Niet-adoptanten rapporteerden over het algemeen een negatievere houding ten opzichte van de verwijshulp dan adoptanten door minder voordelen en meer nadelen te zien, minder sociale steun te ervaren, en een lagere eigen effectiviteit te ervaren. Verder ervaarden zij meer barrières, zoals gebrek aan tijd en gebrek aan vaardigheden. Deze factoren werden ook geassocieerd met de intentie tot adoptie. Aanbevelingen voor toekomstige adoptie zijn onder andere het verbeteren van het instrument zelf door een aanvullende ronde van co-creatie gericht op het verbeteren van de praktische adopteerbaarheid van de verwijshulp. Verdere aanbevelingen hebben betrekking op het communiceren van de toegevoegde waarde van het gebruik van en het verwijzen naar EBSMRIs en het integreren van het gebruik van de verwijshulp in stoppenmet-rokentraining voor ZPs. Dit kan helpen om onder ZPs de attitude, sociale steun, eigen effectiviteit en waargenomen vaardigheden in relatie tot het gebruik van de verwijshulp te verhogen.

\section{Deel II: Toekomstige veranderingen en mogelijkheden}

Hoofdstuk 6 beschrijft de uitkomsten van een scoping-review waarin onderzoek werd gedaan naar literatuur over keuzehulpen die besluitvorming over voeding, lichaamsbeweging, slaap en middelengebruik (waaronder SMR) ondersteunen. De interventies moesten 1) geïnformeerde besluitvorming ondersteunen en 2) informatie verstrekken en helpen om te kunnen kiezen tussen ten minste twee opties. Vijfendertig wetenschappelijke artikelen en vier keuzehulpen werden geïncludeerd, waarvan er 29 (94\%) middelengebruik betroffen. Alle keuzehulpen boden gebruikers informatie en mogelijkheden tot waarde- en/of 


\section{APPENDICES}

voorkeursverheldering. Daarnaast werden ook vele andere elementen aangeboden, zoals doelformulering. In slechts weinig van de geïncludeerde artikelen werd gebruik gemaakt van gestandaardiseerde maten, zoals bijvoorbeeld beslissingsconflict $(n=4 ; 13 \%)$. Hoewel uit het onderzoek slechts enkele positieve gedragseffecten van keuzehulpen bleken, heeft deze studie bijgedragen aan het in kaart brengen van de bestaande besluitvormings- en doorverwijzingshulpmiddelen binnen de gezondheidsbevordering, hun positieve gedragseffecten en de verbeterpunten.

Hoofdstuk 7 beschrijft een vrijstaande usability (gebruiksgemak) studie uitgevoerd in september 2020 op basis van een standalone versie van de verwijshulp (beslissingshulp) onder rokers $(n=497)$ gerekruteerd via een onlineonderzoek panel met behulp van een cross-sectioneeldesign. Het doel van dit onderzoek was drieledig: 1) het uitvoeren van een gebruiksgemakevaluatie van een op zichzelf staande versie van de verwijshulp 2) het evalueren van de mate van waardering en geïnformeerde besluitvorming na gebruik van de verwijshulp en 3 ) het bepalen van een mogelijke verandering in de intentie om EBSMRIs te gebruiken vóór en direct na het beoordelen van de beslissingshulp. De mogelijke veranderingen in de intentie om EBSMRIs te gebruiken tijdens een stoppoging vóór en na het beoordelen van de DA werden getest met t-tests en McNemar. Deelnemers beoordeelden de bruikbaarheid van de beslissingshulp als matig ( $M U ; N=393,79,1 \%$ ) of goed (GU; $N=104$, $20,9 \%$ ). De meeste deelnemers vonden de beslissingshulp slechts matig bruikbaar, deelnemers die de beslissingshulp meer bruikbaar vonden hadden ook vaak een hogere intentie om te stoppen. Deelnemers die de beslissingshulp wel goed bruikbaar vonden, beoordeelden alle elementen betreffende de evaluatie van de beslissingshulp (inclusief de aanbeveling aan anderen en het algemene rapportcijfer) beter en ervaarden minder beslissingsconflicten ten opzien van het kiezen van een potentiele EBSMRIs nadat zij de materialen hadden bekeken. Daarnaast hadden de deelnemers na het doornemen van de beslissingshulp gemiddeld een significant hogere intentie om EBSMRIs te gebruiken, met name in de vorm van eHealth. Aanbevelingen om de beslissingshulp bruikbaarder te maken, waardoor deze beter zal worden ontvangen door een bredere groep rokers, zouden kunnen bestaan uit tailoring op de voorkennis van de gebruikers, het omzetten van op tekst gebaseerde informatie in op video gebaseerde informatie en het opnemen van methoden voor het verhelderen van de waarde. Omdat de beslissingshulp slechts matig bruikbaar bleek in de standalone versie, zou een hybride variant waarbij rokers de beslissingshulp zowel zelfstandig als met begeleiding van een ZP kunnen gebruiken, beide groepen mogelijk kunnen helpen bij het kiezen van een passende EBSMRI.

Ten slotte bespreekt hoofdstuk 8 de resultaten gepresenteerd in de hoofdstukken 2-7 van dit proefschrift. Ook bevat dit hoofdstuk een aantal overwegingen van de hierboven beschreven studies inclusief aanbevelingen voor toekomstig onderzoek en de 
uitvoeringspraktijk. Het doel van het onderzoek was om een RA (referral aid, verwijshulp) te ontwikkelen om ZPs te helpen bij het verwijzen naar EBSMRIs om zo het gebruik van EBSMRIs onder rokers te verhogen. De studies beschreven in dit proefschrift laten zien dat de RA goed werd gewaardeerd door ZPs en dat het gebruik ervan leidde tot iets meer discussie en doorverwijzing naar EBSMRIs. De RA werd ook gewaardeerd door rokers, vooral door degenen die gemotiveerd zijn om te stoppen met roken. Hoewel de verwijshulp een breed scala aan EBSMRIs introduceerde, leidde dit niet tot beslissingsconflicten onder de gebruikers. Er konden echter geen effecten op rookabstinentie worden gemeld. Dit werd hoogstwaarschijnlijk veroorzaakt door het feit dat beide condities evidence-based counseling ontvingen van hun $\mathrm{POH}$ en door de kleine studiesteekproef. Verder werd de verwijshulp niet intensief gebruikt tijdens de RCT, wat waarschijnlijk werd veroorzaakt door barrières zoals gebrek aan tijd en een hoge werkdruk. In dit hoofdstuk hebben we een aantal overwegingen besproken met betrekking tot het vergemakkelijken van de werving van ZPs en rokende patiënten en hun therapietrouw aan de verwijshulp. We hebben ook enkele aanbevelingen gedaan voor verder onderzoek met betrekking tot (1) de ZPs (bijv. mogelijk verhogen van deelname en verbeteren van implementatie); (2) de verwijshulp beschreven in dit proefschrift (bijv. ontwikkelen van een hybride variant en aanvullen van de verwijshulp met verschillende vormen van motiverende technieken, tailoring, video-gebaseerde materialen, en waarde-verklaringsoefeningen); en (3) stoppen met roken in het algemeen (aanspreken van het zorgverzekeringssysteem en beleidsmakers en het verbeteren van de beschikbaarheid van EBSMRIs).

Om het aantal gegeven stopadviezen in de eerstelijnszorg te verhogen en de verwijshulp in de dagelijkse routine van de ZPs (voornamelijk POHs) te implementeren, zien we mogelijkheden om de verwijshulp te implementeren in de praktijk, bijvoorbeeld binnen het verwijsonderdeel van de strategie Ask-Advize-Refer (AAR: vraag, adviseer, verwijs). Op deze manier kan de verwijshulp dienen om de beschikbare EBSMRIs aan rokers uit te leggen tijdens de verwijsfase, maar ook als een referentie die rokers zelf kunnen raadplegen na het gesprek met de ZP, wat de actieve begeleidingstijd kan verminderen. Om de implementatie te stimuleren, zou de RA daarom kunnen worden opgenomen in nationale stoppen met roken trainingen gericht op $\mathrm{POHs}$. Verder kan de ontwikkeling van een hybride variant het gebruik van de RA ondersteunen ter voorbereiding op een consult met een ZP of de roker in staat stellen een eigen keuze te maken met betrekking tot het gebruik van EBSMRIs in het geval de tool online wordt aangeboden als een zelfhulp tool. 


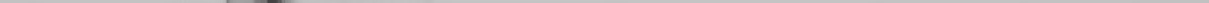




\section{CURRICULUM VITAE}

Daniëlle Nicole Zijlstra was born on the 23rd of October 1993 in Hoogeveen, the Netherlands. After graduating high school (Pre-University Education; Culture and Society) at the Roelof van Echten College in 2012, she studied Psychology (Bachelor) at the University of Twente, from which she graduated in 2015. She subsequently studied Positive Psychology and Technology (Master) until July 2016.

In January 2017, Daniëlle started working as a PhD-student at the Department of Health Promotion at Maastricht University. For four and a half years she worked on several studies regarding the StopWijzer-project that are described in this dissertation. Besides her work on the StopWijzer-project, she has also been actively involved in teaching activities in the Bachelor of Health Sciences and the Master of Health Education and Promotion. These teaching experiences led her to receive the Basic Teaching Qualification Certificate.

Daniëlle currently continues to work as a full-time teacher in various educational roles (tutor, mentor, trainer, course planning member) at the Department of Health Promotion at Maastricht University. She aspires to further develop her teaching and mentoring skills as well as to focus on educational research and development. 


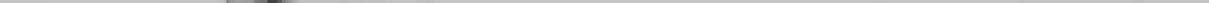




\section{PUBLICATION LIST}

\section{Publications in this thesis}

Zijlstra, D. N., Hoving, C., Bolman, C. A. W., Muris, J. W. M. \& De Vries, H. (2021). Do professional perspectives on evidence-based smoking cessation methods align? A Delphi study among researchers and healthcare professionals. Health Education Research. https:// doi.org/10.1093/her/cyab022

Zijlstra, D. N., Muris, J. W. M., Bolman, C. A. W., Elling, J. M., Knapen, V. E. R. A. \& De Vries, H. (2021). A referral aid for smoking cessation interventions in primary care: Study protocol for a randomized controlled trial. Primary Health Care Research \& Development, 22, E22. https://doi.org/10.1017/S1463423621000244

Zijlstra, D. N., Muris, J. W. M., Bolman, C. A. W. \& De Vries, H. (2021). What went wrong? A randomized controlled trial of a process and effect evaluation of a referral aid for smoking cessation counseling in primary care. Manuscript submitted for publication.

Zijlstra, D. N., Bolman, C. A. W., Muris, J. W. M. \& De Vries, H. (2021). How to convince more primary care professionals to adopt a valued smoking cessation tool: Facilitators and barriers. Manuscript submitted for publication.

Gültzow, T., Zijlstra, D. N., Bolman, C. A. W., de Vries, H., Dirksen, C. D., Muris, J. W. M., Smit, E. S., \& Hoving, C. (2021). Decision aids to facilitate decision making around behavior change in the field of health promotion: A scoping review. Patient Education and Counseling. https://doi.org/10.1016/j.pec.2021.01.015

Zijlstra, D. N., Bolman, C. A., Muris, J. W., \& de Vries, H. (2021). The Usability of an Online Tool to Promote the Use of Evidence-Based Smoking Cessation Interventions. International Journal of Environmental Research and Public Health, 18(20), 10836. https://doi.org/10.3390/ ijerph182010836

\section{Presentations at (inter)national congresses}

Care and Public Health Research Institute (CAPHRI) Research Day, 2017, Maastricht, Nederland

Poster: Making your own choice - A study on smoking cessation among smokers with smoking related complains. 
16th International Conference on Communication in Healthcare (ICCH), 2018, Porto, Portugal

Presentatie: General Practice Professional needs and expectations regarding a patient smoking cessation support

Poster: An integral Pro-Active Multicomponent Approach (PAMA) to optimize and tailor smoking cessation strategies for the primary health care $(\mathrm{PHC})$ setting

Nederlands Netwerk voor Tabaksonderzoek (NNvT) congres, 2018, Utrecht, Nederland Presentatie: Delphi studie naar consensus tussen onderzoekers en zorgprofessionals over evidence-based stoppen met roken interventies in de huisartspraktijk

\section{ZonMW Werkconferentie, 2018, Utrecht, Nederland}

Presentatie: An integral Pro Active Multicomponent Approach (PAMA) to optimize and tailor smoking cessation strategies for the primary health care $(\mathrm{PHC})$ setting

COPD \& Astma Huisartsen Advies Groep (CAHAG) conferentie, 2019, Zeist, Nederland Presentatie: StopWijzer - De stoppen-met-roken keuzetool voor in de Huisartspraktijk

Nederlands Netwerk voor Tabaksonderzoek (NNvT) congres, 2019, Utrecht, Nederland Presentatie: Het stimuleren van verwijzingen naar effectieve counseling door de huisartspraktijk (onderdeel van symposium: Never change a winning team? Of blijft het belangrijk om nieuwe manieren te zoeken om tabaksgebruik aan te pakken?) 


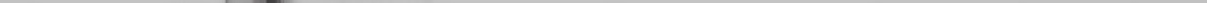




\section{DANKWOORD}

Hoewel ik al veel eerder heb geroepen dat ik er helemaal klaar mee was, is mijn dissertatie nu toch ook echt af. Ik wil graag iedereen bedanken die er op wat voor wijze dan ook aan heeft bijgedragen dat het na 4,5 jaar knokken eindelijk zover is. Natuurlijk zijn er ook een aantal mensen die ik in het bijzonder wil bedanken.

Hein. Jij zat altijd vol out-of-the-box ideeën, ik snap niet hoe je dat doet. Van jou heb ik geleerd dat ik veel meer aan kan dan ik dacht en dat er altijd en plan b is (en een plan $c, d$, e, f enz.).

Catherine. Ik heb ontzettend veel respect voor jouw oog voor detail en de drive en passie die jij hebt voor je werk. Ontzettend bedankt dat ik altijd op jouw input heb mogen rekenen.

Jean. Ondanks alle hectiek op het project leek jij je hoofd altijd kalm te kunnen houden. Dit heeft mij ontzettend geholpen op de momenten dat dat mij zelf niet meer lukte, bedankt hiervoor.

Leden van de leescommisie en de corona. Uiteraard wil ik ook mijn dank uitspreken richting jullie vanwege de tijd die jullie hebben gestoken in het beoordelen van het proefschrift en jullie bereidheid om bij mijn verdediging een rol te spelen.

Uiteraard ook bedankt aan alle deelnemers aan de in dit proefschrift beschreven onderzoeken, met name de praktijkondersteuners en de verschillende organisaties die hebben meegewerkt aan het project.

Ciska. Ook jou ben ik natuurlijk niet vergeten. Ik wil je ontzettend bedanken voor de ondersteuning die je me hebt gegeven.

Vera, Raesita, Merel, Thomas 0., Myrthe en Eline, mijn small army aan assistenten. Bedankt voor jullie inzet gedurende verschillende (soms wat hectische) periodes van het project.

Alle (ex-)GB-collegas die ik de afgelopen jaren ben tegengekomen. Hoewel jullie me bijna uit Limburg hadden weggejaagd na de eerste keer carnaval, hebben we het ook ontzettend leuk gehad. Ik hoop we elkaar snel weer eens tegenkomen op een plek die niet ZOOM is.

Ook een extra bedankje naar Denise, Daisy, Kim, Patricia en Leon voor alles waar ze de afgelopen jaren bij geholpen hebben en een speciale shout-out naar Kathelijne door wie ik mijn interesse voor het onderwijs heb ontdekt. 
Thomas. Bitte lass uns trotzdem Freunde bleiben, obwohl wir uns manchmal nicht können leiden. Ik zal jou en al je 'gedoe' nog eens een keer gaan missen als ik in m'n eentje op kantoor zit. Joe, bedankt.

Karin. Bedankt voor alle keren dat je me geruststelde op de momenten dat ik weer eens riep nu echt een one-way-ticket naar IJsland te boeken en nooit meer terug te komen. Door jou wist ik wat ik kon verwachten en hoe ik dit kon handelen.

Iris. Mensen die onze whatsapp geschiedenis zouden doorlezen zouden ons allebei voor gek verklaren. Bedankt dat je al die tijd mijn geklaag, geraaskal en andere onzin hebt aangehoord en er toch nog elke dag voor me bent.

Yil. Hoewel we soms geen bal van elkaar begrijpen, kunnen we het toch altijd heel goed met elkaar vinden. Bedaank voor je luisterend oor, je hulp bij het schrijven van teksten en natuurlijk alle gezelligheid. Ik denk dat het wel weer tijd is voor een wijntje samen.

Mathis. Je was echt een vaste rots in het proces omdat je je (ogenschijnlijk) nooit laat gek maken. Soort nuchtere tukker maar dan anders. Daar kan ik echt nog wel wat van leren. Ik kijk uit naar nog vele cola zero's en borden patat met jou.

Papa en Mama. Bedankt voor jullie onvoorwaardelijke steun al mijn gehele leven. Het is ontzettend fijn om te weten dat ik altijd bij jullie terecht kan.

Nico. Van heel dichtbij, naar heel ver weg naar weer nog dichterbij. Je bent me gevolgd toen ik zo nodig naar de andere kant van Nederland moest. lk weet niet waar we hierna zullen uitkomen, maar we komen er vast en zeker samen wel uit :) 

